\title{
CIN85/CD2AP-based protein complexes in $B$ cell antigen receptor signalling
}

\author{
Doctoral Thesis \\ In partial fulfilment of the requirements for the degree \\ "Doctor rerum naturalium (Dr. rer. nat.)" \\ in the Molecular Medicine Study Program \\ at the Georg-August University Göttingen
}

submitted by

Vanessa Bremes

born in

Düsseldorf, Germany

Göttingen, May 2012 


\section{Members of the thesis committee}

\section{Supervisor:}

Prof. Dr. Jürgen Wienands

Department of Cellular and Molecular Immunology

Georg-August University Göttingen

Second member of the thesis committee:

Prof. Dr. Henning Urlaub

Bioanalytical Mass Spectrometry Group

Max-Planck-Institute for Biophysical Chemistry Göttingen

Third member of the thesis committee:

Dr. Dieter Klopfenstein

Third Institute of Physics - Biophysics

Georg-August University Göttingen

Date of Disputation: 


\section{Affidavit}

Here I declare that my doctoral thesis entitled

"CIN85/CD2AP-based protein complexes in B cell antigen receptor signalling" has been written independently with no other sources and aids than quoted.

Vanessa Bremes

Göttingen, May 2012 


\section{List of publications}

2011

Thomas Oellerich, Vanessa Bremes, Konstantin Neumann, Hanibal Bohnenberger, Kai Dittmann, He-Hsuan Hsiao, Michael Engelke, Tim Schnyder, Facundo D Batista, Henning Urlaub, and Jürgen Wienands (2011).

"The B cell antigen receptor signals through a preformed transducer module of SLP65 and CIN85".

EMBO J. (17):3620-3634.

Participation at scientific meetings:

Poster $3^{\text {rd }}$ EU Summer School in Proteomics, Brixen, Italy

2010

Poster $8^{\text {th }}$ B Cell Meeting of the German Society of Immunology, Dresden, Germany

2011

Oral Retreat of the Collaborative Research Centre

"Integrative Structural Biology of Dynamic Macromolecular Assemblies", Drübeck, Germany 


\section{Acknowledgements}

I would like to express my gratitude to Prof. Dr. Jürgen Wienands for accompanying me over the past years as my "Doktorvater". His motivating support, our constructive dialogues and his competent advice, have made important contribution to my development as a scientist and this $\mathrm{PhD}$ thesis.

I also want to acknowledge the members of my thesis committee, Dr. Dieter Klopfenstein and Prof. Dr. Henning Urlaub, for their interest in my project and their comments during thesis committee meetings. I also like to thank the Molecular Medicine PhD Program.

I sincerely thank all authors of our publication in the EMBO journal, especially, Thomas, Kontstantin, Hanibal, Michael and Kai from our group for their individual contribution to the success of this story. In addition, I would like to thank our collaborators Tim Schnyder and Dr. Facundo Batista for performing TIRF microscopy as well as He-Hsuan Hsiao and Prof. Dr. Henning Urlaub for mass spectrometry. Further, I thank Prof. Dr. Carsten Lüder for providing me with access to the confocal microscope in his department.

I express my gratitude to Gabi, Ines and Leslie for their help to smoothly run the lab. In particular, I thank Gabi for technical assistance and the generation of antisera. Additionally, I would like to thank our secretaries Ingrid Teuteberg and Rosemarie Döhne for brighten up each day with an unmistakable laughter.

Thanks to all current and former members of our department for help, discussions and good times. Especially, many, many thanks to my "Frösche, Freunde, Kameraden" Johannes, Niklas and Lars for making the most of our daily lunch in the Mensa!!! I really enjoyed spending time with you and am grateful for your support even through the harder phases of the last years. Thank you Lars for discussions about RNAi approaches. In addition I thank Wiebke and Henrike for sharing coffee, cakes and wonderful dinners - and @ Henrike even hair-cut, glasses and clothes.

I also would like to thank my students Jana and Kristin, whose efforts have contributed to this thesis.

Furthermore, I would like to thank Niklas and Nils for critically reading this thesis.

I thank the German Research Foundation and the Medical Faculty of the Georg-August University of Göttingen for financial support. 
I am very grateful for having good friends in my life. Wiebke and Tine, thank you for inspiring, in-depth discussions about live in general on Tuesday evenings. Tine, thanks for all the great times in our wounderful student's home! Yvi, Rachel, Didi and Chrissi you are my friends since such a long time now and I thank you for always being at my side.

Finally, I would like to thank my family for unconditional support and for having built a true home, in which I always feel at ease. Dear Nils I thank you for being at my side and for the way you encouraged me during the downs and enjoyed life with me during the ups of the last years. 


\section{Content}

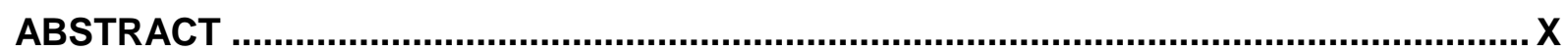

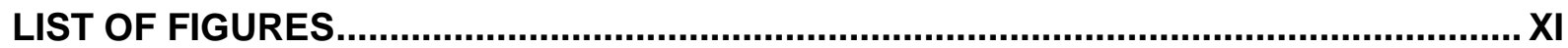

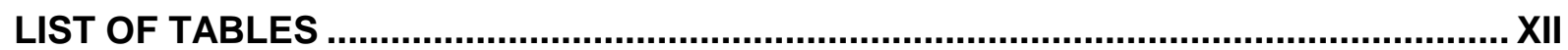

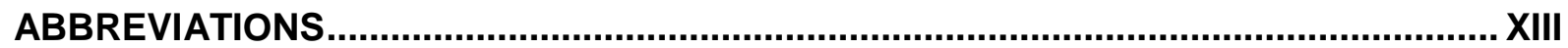

AMINO ACIDS AND DEOXYRIBONUCLEOTIDES ……...............................................

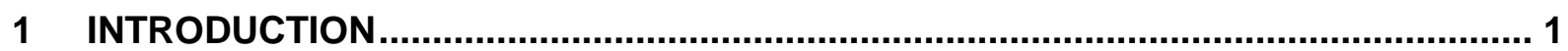

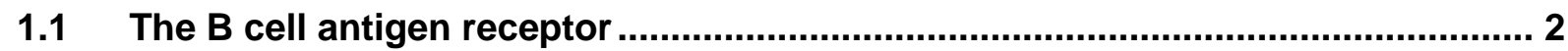

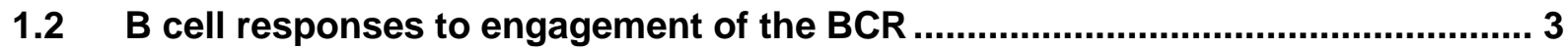

1.3 The BCR signalling cascade leading to a $\mathrm{Ca}^{2+}$ response.................................... 4

1.3.1 The BCR-induced protein phosphorylation cascade ....................................... 5

1.3.2 The two phases of the BCR-triggered $\mathrm{Ca}^{2+}$ response .................................. 6

1.4 The adaptor protein SLP65 and its steady ligands CIN85 and CD2AP ................ 7

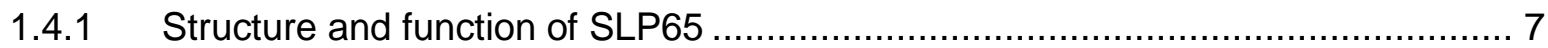

1.4.2 Membrane recruitment of SLP adaptors ................................................... 8

1.4.3 The scaffolds CIN85 and CD2AP ............................................................. 9

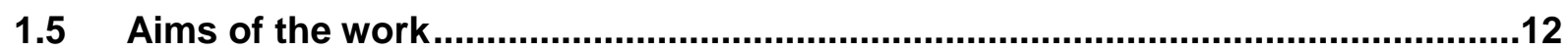

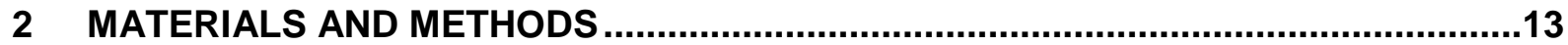

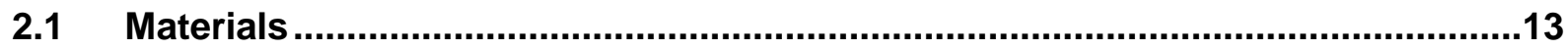

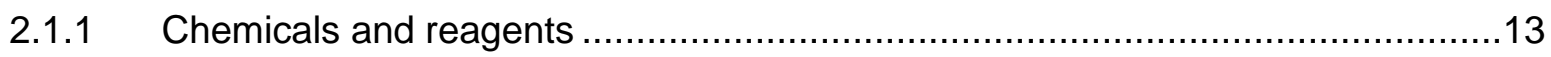

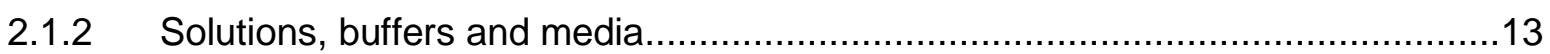

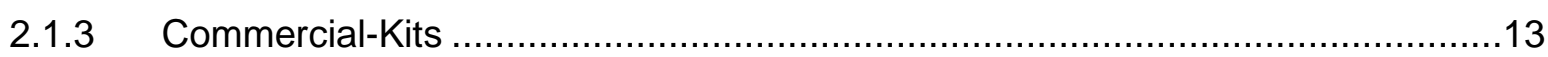

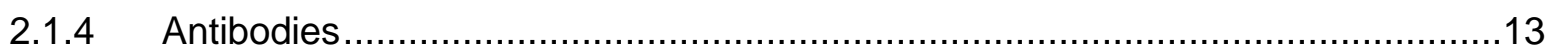

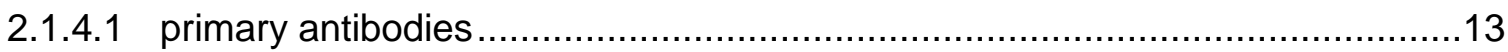

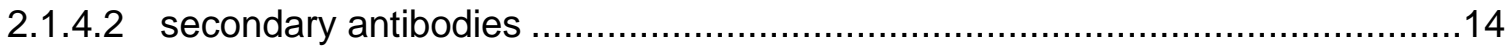

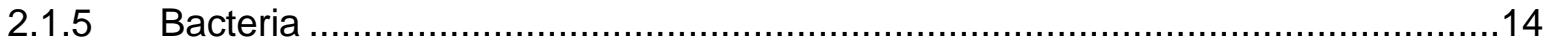

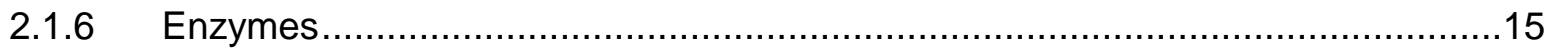




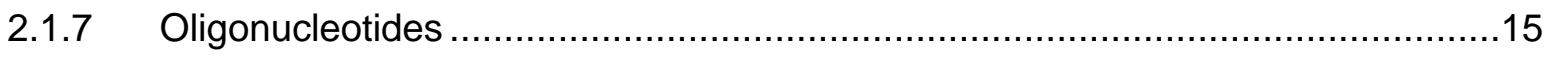

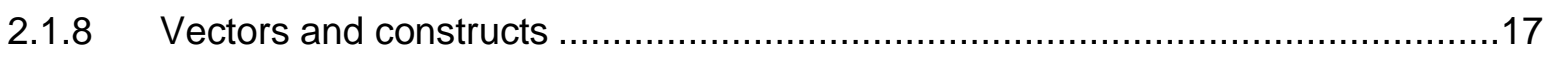

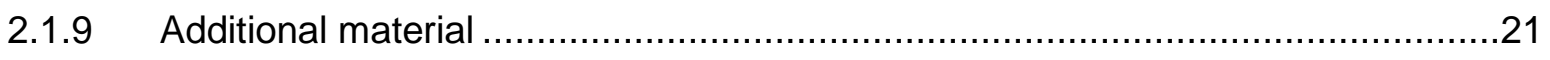

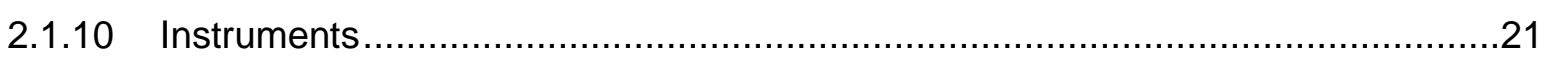

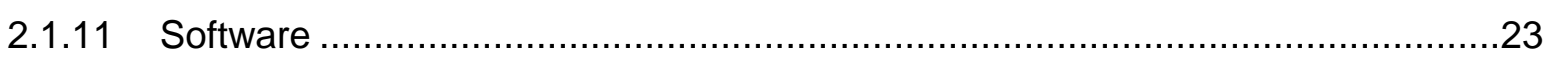

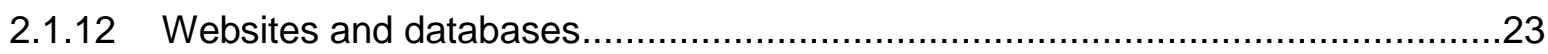

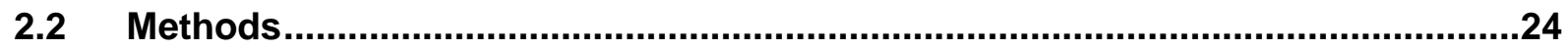

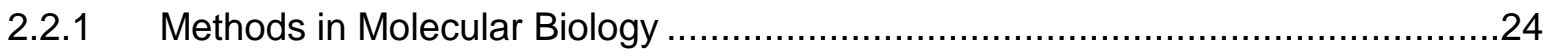

2.2.1.1 Fragmentation of DNA with restriction endonucleases (typell) ......................24

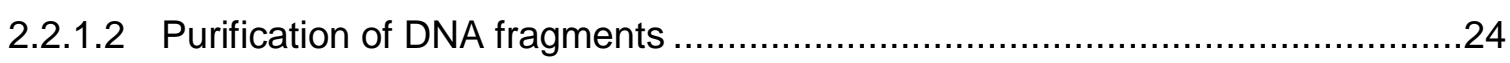

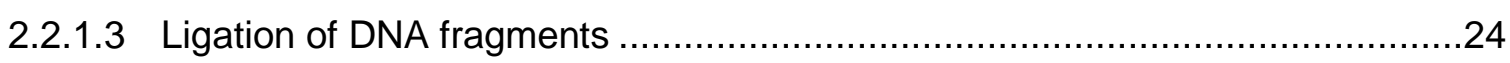

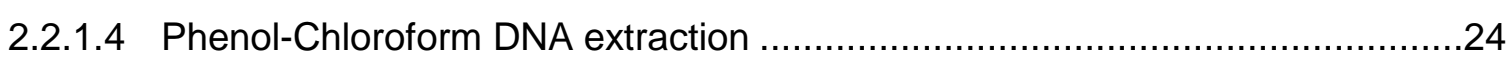

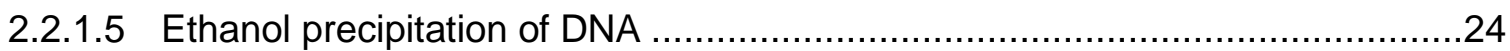

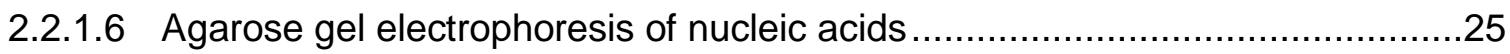

2.2.1.7 Photometric determination of DNA concentration .......................................25

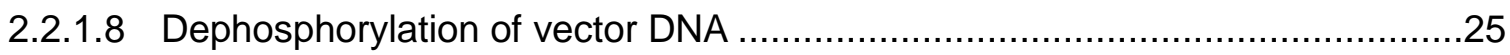

2.2.1.9 Extraction and purification of plasmid DNA from E.coli ..................................25

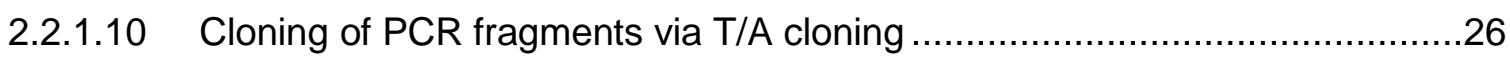

2.2.1.11 Isolation of genomic DNA from DT40 B cells .........................................26

2.2.1.12 Preparation of chemo-competent E.coli ................................................26

2.2.1.13 Transformation of chemo-competent E.coli................................................27

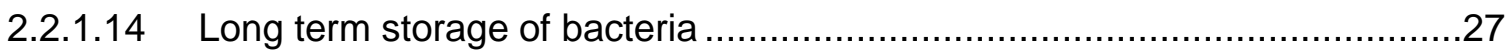

2.2.1.15 In vitro amplification of DNA by the Polymerase Chain Reaction (PCR) ......27

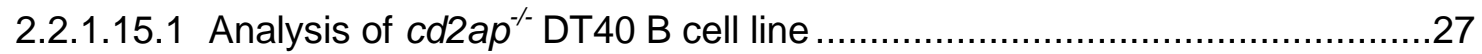

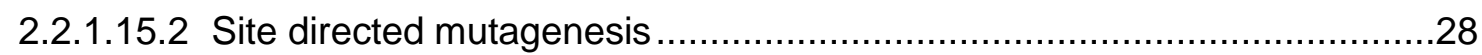

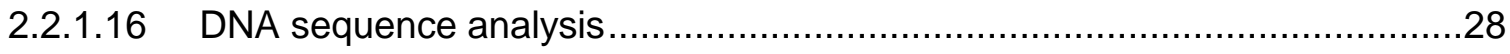

2.2.1.17 RNA interference (RNAi) with cin85 expression using microRNA-30 adapted shRNAmir retroviral vectors ...............................................29

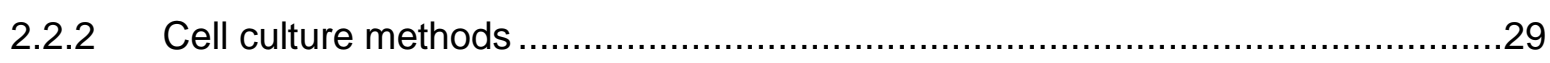

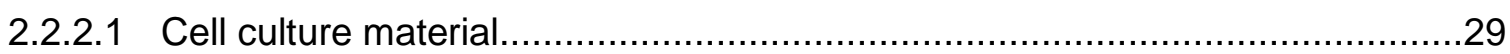

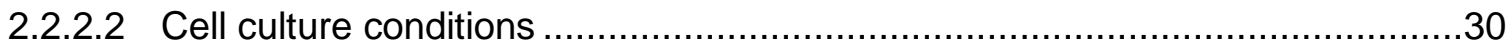

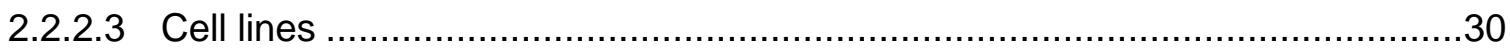

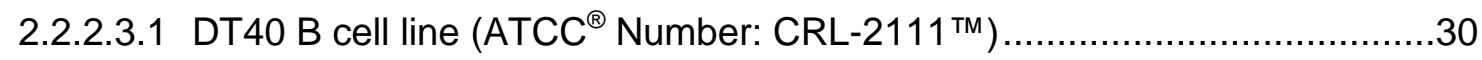

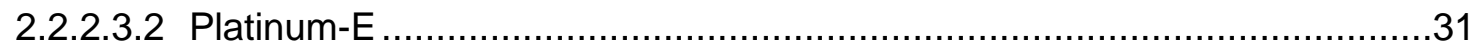

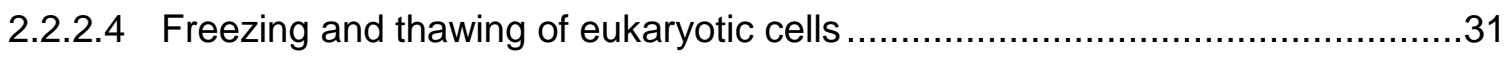

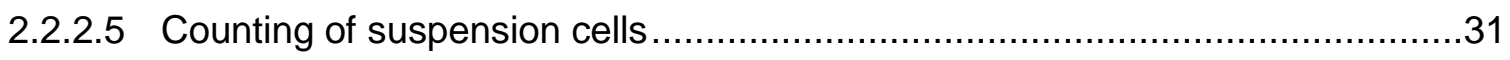

2.2.2.6 Transfection of DT40 B cells by electroporation ....................................... 
2.2.2.7 Transfection of Platinum-E cells by lipofection.............................................

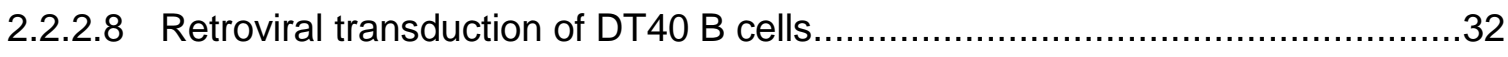

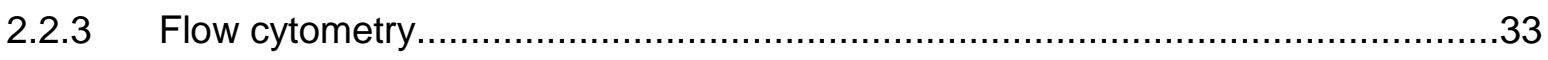

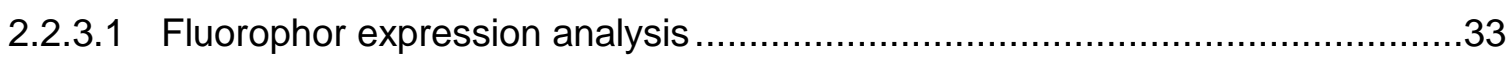

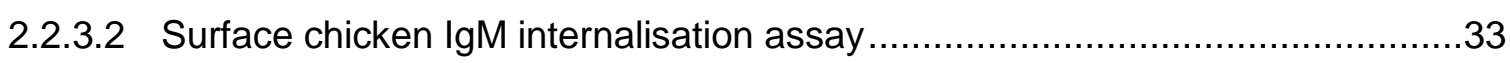

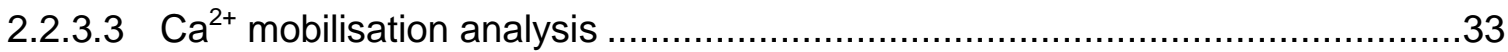

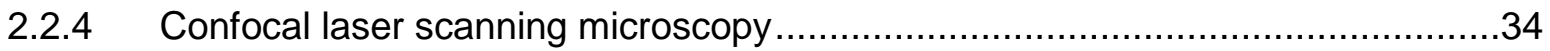

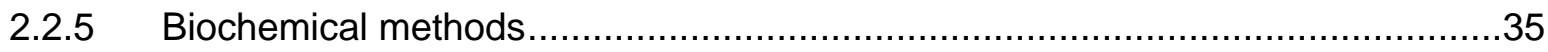

2.2.5.1 Expression and purification of recombinant GST fusion proteins ....................35

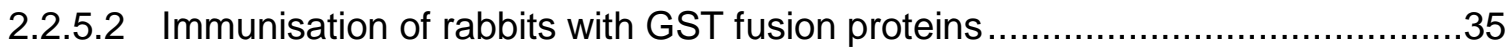

2.2.5.3 Expression and purification of recombinant $\mathrm{His}_{6}$ fusion proteins ......................36

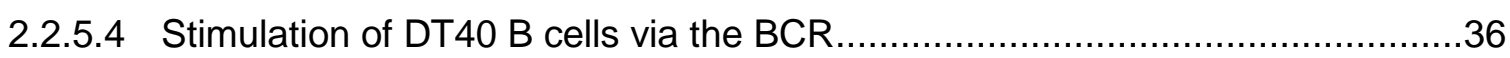

2.2.5.5 Affinity purification with GST fusion proteins or antibodies ..............................37

2.2.5.6 SDS-polyacrylamide gel electrophoresis (SDS-PAGE) …...........................

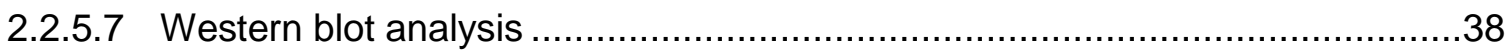

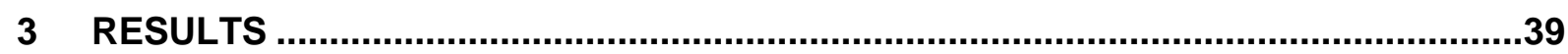

3.1 CIN85 is a positive regulator in the onset of $\mathrm{BCR}$-induced $\mathrm{Ca}^{2+}$ signalling,

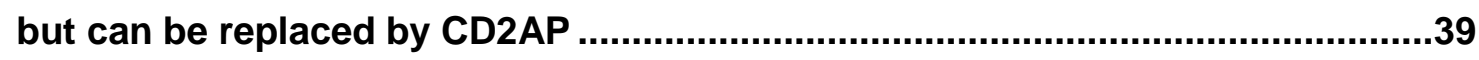

3.1.1 CIN85 has a positive regulatory role in BCR-induced $\mathrm{Ca}^{2+}$ flux .........................39

3.1.2 CD2AP is dispensable for BCR-induced $\mathrm{Ca}^{2+}$ mobilisation .............................41

3.1.3 Reduced expression of cin85 combined with CD2AP-deficiency impaired BCR-induced $\mathrm{Ca}^{2+}$ mobilisation................................................................4 44

3.1.4 Ectopic re-expression of cin85 reverses the defect in the onset of BCR-induced $\mathrm{Ca}^{2+}$ mobilisation in cin85 ${ }^{\text {sh }}$ DT40 B cells .46

3.1.5 CD2AP can rescue the BCR-triggered $\mathrm{Ca}^{2+}$ mobilisation defect induced by diminished cin85 expression

\subsection{The preformed complex of SLP65 and CIN85/CD2AP is based on the} interaction of the SH3 domains of CIN85/CD2AP and atypical proline-arginine motifs in SLP65

3.2.1 The SH3 domains of CIN85 and CD2AP bind to atypical proline-arginine motifs in SLP65.

3.2.2 CIN85 and CD2AP bind the second and third atypical proline-arginine

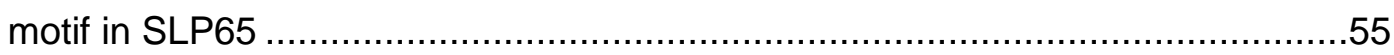

3.2.3 CIN85 and CD2AP can hetero-oligomerise via coiled coil domains.....................56

3.2.4 CIN85 and CD2AP bind to SLP65 independent of each other............................56 
3.3 CIN85 and CD2AP are recruited to the plasma membrane in

BCR-activated B lymphocytes using different anchoring modes

3.4 The SH3 and coiled coil domains of CIN85 mediate both

BCR-induced membrane recruitment and $\mathrm{Ca}^{2+}$ flux

3.4.1 The $\mathrm{SH} 3$ and coiled coil domains of CIN85 are indispensable for full $\mathrm{Ca}^{2+}$ signalling in BCR-activated cells

3.5 CIN85 colocalises with BCR-containing microclusters 65

3.5.1 CIN85 colocalises with BCR-containing microclusters independent of SLP65 ...65

3.5.2 BCR internalisation is not affected by reduced cin85 expression

3.6 Lyn, but not Syk kinase activity is required for BCR-induced plasma membrane translocation of CIN85

3.6.1 The BCR-induced recruitment of CIN85 to the plasma membrane requires Lyn, but not Syk .70

3.6.2 Membrane recruitment of CIN85 after BCR stimulation is independent of CD2AP or Grb2.

3.7 BCR-targeted SLP65 functions independent of CIN85/CD2AP in

BCR-triggered $\mathrm{Ca}^{2+}$ mobilisation

3.7.1 The TIRAP PIP 2 binding domain substitutes the function of the N-terminus in SLP65_wt, but not in SLP65_M23.

3.7.2 Targeting of SLP65_M23 to the BCR restores its function in BCR-induced $\mathrm{Ca}^{2+}$ mobilisation

4 DISCUSSION.

4.1 CIN85, CD2AP and SLP65 - complex BCR signalling

4.1.1 The preformed complex of CIN85 and SLP65 in BCR-induced

$\mathrm{Ca}^{2+}$ mobilisation

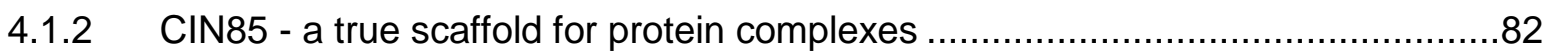

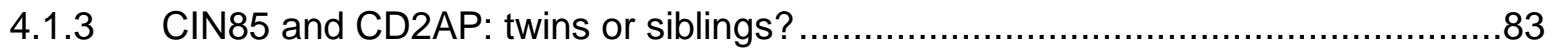

4.2 SLP65's third tooth for biting the membrane ...................................................88

4.2.1 Sub cellular localisation of CIN85 and CD2AP ...........................................88

4.2.2 CIN85 - a novel resident of BCR-containing microcluster ................................90

4.2.3 CIN85 and SLP65 - a preformed and BCR-targeted module ............................92

4.3 Outlook 
5 SUMMARY AND CONCLUSIONS.

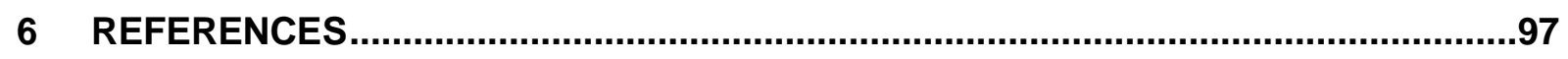

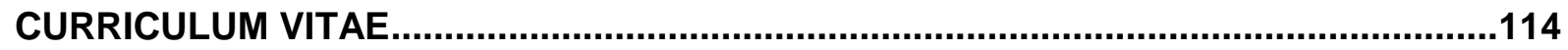




\section{Abstract}

Early $\mathrm{B}$ cell antigen receptor (BCR) signal transduction involves formation of multimeric protein complexes and their recruitment to BCR-containing areas in the plasma membrane. One such membrane-targeted signalosome is the $\mathrm{Ca}^{2+}$ initiation complex that is assembled on the central adaptor protein SH2 domain-containing leukocyte protein of $65 \mathrm{kDa}$ (SLP65). To decipher the mechanism of SLP65 membrane localisation, the interactome of SLP65 was analysed by our group and identified the scaffolds Cbl-interacting protein of $85 \mathrm{kDa}$ (CIN85) and CD2-associated protein (CD2AP) to be constantly associated with SLP65. Further studies showed that disruption of this interaction by changes of SLP65 binding motifs resulted in impaired $\mathrm{BCR}$-induced $\mathrm{Ca}^{2+}$ mobilisation in DT40 and primary murine $\mathrm{B}$ cells.

Hence, the following questions arose for this thesis: Does ablation of CIN85 and CD2AP result in a BCR-induced $\mathrm{Ca}^{2+}$ mobilisation defect as shown on the SLP65 side and, thus, do CIN85, CD2AP and SLP65 comprise a preformed signal transducer complex? And, how do CIN85 and CD2AP then contribute to SLP65 function in BCR signal transduction?

In my thesis, I demonstrated that CIN85 positively regulated the rapid onset and strength of BCR-induced $\mathrm{Ca}^{2+}$ mobilisation in DT40 B cells. However, CD2AP could partially replace CIN85 function in this respect. Using live cell imaging I showed that CIN85 and CD2AP translocate to the plasma membrane upon stimulation of the BCR, but used different anchoring modes. Interestingly, the very same domains of CIN85 necessary for BCRinduced $\mathrm{Ca}^{2+}$ signalling provided CIN85 with access to the plasma membrane. More detailed analysis of membrane localisation using total internal reflection microscopy identified CIN85 to colocalise with BCR-containing microclusters upon BCR engagement. In contrast to CIN85 only very few CD2AP molecules were found in these microclusters. Importantly, the preformation of CIN85 with SLP65 could be bypassed by providing SLP65 with direct access to the BCR. This implies that CIN85 and SLP65 comprise a preformed BCR transducer module. In this complex CIN85 targets SLP65 directly to BCR-containing microclusters thereby enabling efficient $\mathrm{BCR}$-induced $\mathrm{Ca}^{2+}$ responses. Collectively, the herein documented results contribute to the understanding of $\mathrm{BCR}$ activation with respect to BCR-proximal signalosomes and their importance in the transduction of BCR signals. 


\section{List of figures}

Figure 1.1: BCR-induced signalling events leading to a rise in intracellular $\mathrm{Ca}^{2+}$ concentration.

Figure 1.2: SLP65 domain architecture modified from (Koretzky et al. 2006).

Figure 1.3: Domain architecture of CIN85 and CD2AP modified from (Tibaldi and Reinherz 2003).

Figure 3.1: Both CIN85 isoforms at $80 \mathrm{kDa}$ are targeted by the shRNA in DT40 B cells. 40

Figure 3.2: CIN85 is a positive regulator of BCR-induced $\mathrm{Ca}^{2+}$ flux.

Figure 3.3: Generation of a cd2ap $\%$ DT40 B cell line. .42

Figure 3.4: CD2AP is dispensable for BCR-induced $\mathrm{Ca}^{2+}$ mobilisation. .44

Figure 3.5: $\mathrm{BCR}$-induced $\mathrm{Ca}^{2+}$ mobilisation is impaired upon reduced cin85 expression and CD2AP-deficiency.

Figure 3.6: Experimental strategy for the reconstitution of cin85/cd2ap expression in shRNA transfected DT40 B cells.

Figure 3.7: Ectopic re-expression of cin85 reverses the defect in $\mathrm{BCR}$-induced $\mathrm{Ca}^{2+}$ mobilisation of cin85 $5^{\text {sh }}$ DT40 B cells.

Figure 3.8: The strength of ectopic cin85 expression influences the BCR-induced $\mathrm{Ca}^{2+}$

profile. .50

Figure 3.9: CD2AP can rescue the $\mathrm{Ca}^{2+}$ mobilisation defect induced by diminished $\operatorname{cin} 85$ expression. 52

Figure 3.10: The SH3 domains of CIN85 and CD2AP bind to atypical proline-arginine motifs in SLP65.

Figure 3.11: CIN85 and CD2AP preferentially bind the second atypical proline-arginine motif in SLP65. .55

Figure 3.12: CIN85 and CD2AP can hetero-oligomerise via coiled coil domains. .56

Figure 3.13: CIN85 and CD2AP bind to SLP65 independent of each other. .57

Figure 3.14: CIN85 and CD2AP translocate to the plasma membrane after stimulation of the

$\mathrm{BCR}$, but use different anchoring modes. .58

Figure 3.15: The SH3 domains of CIN85 are necessary domains for $\mathrm{Ca}^{2+}$ mobilisation in BCR-stimulated DT40 B cells.

Figure 3.16: The coiled coil domain of CIN85 exerts a function in BCR-induced $\mathrm{Ca}^{2+}$ flux...64 Figure 3.17: CIN85 colocalises with BCR-containing microclusters independent of SLP65. .67

Figure 3.18: CIN85 associates with surface $\operatorname{lgM}$. .68

Figure 3.19: Reduction of cin85 expression has no influence on BCR internalisation. 69

Figure 3.20: The BCR-induced membrane recruitment of CIN85 requires Lyn, but not Syk..72 
Figure 3.21: BCR-induced membrane recruitment of CIN85 is independent of CD2AP or

Grb2

Figure 3.22: The TIRAP PIP 2 binding domain functionally substitutes the $\mathrm{N}$-terminus of

SLP65_wt, but not SLP65_M23.

Figure 3.23: Targeting of SLP65_M23 to the BCR restores its function in $\mathrm{Ca}^{2+}$ mobilisation after BCR cross-linking. .78

Figure 4.1: Model for the interaction of CIN85 or CD2AP with SLP65. .86

Figure 4.2: Model for the mechanisms of CIN85 and CD2AP plasma membrane recruitment

Figure 4.3: Model of the preformed, CIN85/SLP65 BCR transducer module in the initiation of $\mathrm{Ca}^{2+}$ signalling. .93

\section{List of tables}

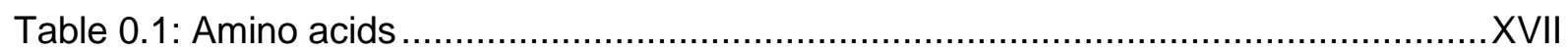

Table 0.2: Deoxyribonucleotides .........................................................................

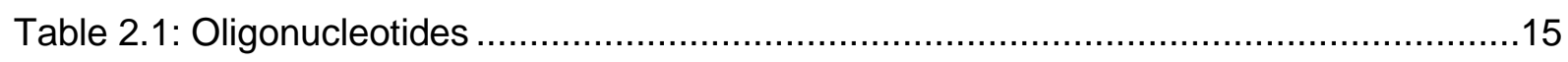

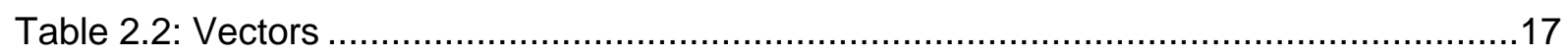

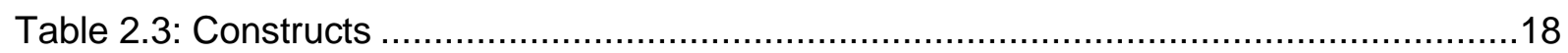

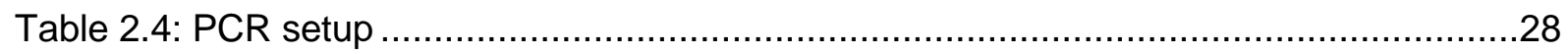

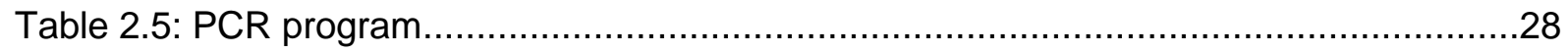

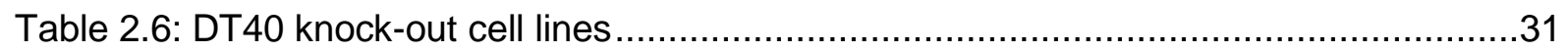

Table 3.1: Quantification of plasma membrane localisation of CIN85 or CD2AP and their respective variants in resting or BCR-activated DT40 B cells ..................................60

Table 3.2 Quantification of plasma membrane localisation of CIN85 in different DT40 B cell lines.

Table 3.3 Quantification of plasma membrane localisation of CIN85 in cd2ap ${ }^{\%}$ or grb2

DT40 B cell lines before and after BCR stimulation

Table 3.4: Quantification of the membrane localisation of the TIRAP-SLP65 chimeras .76

Table 3.5: Quantification of the plasma membrane localisation of the tSH2_SLP65 chimeras

Table 4.1 Structural and functional features of CIN85 and CD2AP 87 


\section{Abbreviations}

$\Delta$

$\mu$ aa

AM

Amp

AP

APC

APS

ATCC

ATP

BCR

blast

bleo

bp

bs

BSA

Btk

CapZ

Cbl

CC

$\mathrm{CCL}$

CD2

CD2AP

CD2BP3

cDNA

ch

CIN85

cin85

CIP

cit

CRAC

CS

$\mathrm{Da}$

DAG

DMEM deletion/truncation

micro

amino acid

acetylmethyl (ester form)

ampicillin

affinity purification

antigen-presenting cell

ammonium persulphate

American type culture condition

adenosine trisphosphate

$B$ cell antigen receptor

blasticidin resistance

bleomycin resistance

base pair

blasticidin $\mathrm{S}$

bovine serum albumin

Bruton`s tyrosine kinase

F-actin capping protein

Casitas B-lineage lymphoma

coiled coil

cleared cellular lysates

cluster of differentiation 2

CD2-associated protein

CD2 binding protein; isoform of CIN85

complementary DNA

chicken

Cbl-interacting protein of $85 \mathrm{kDa}$

DT40 B cells expressing shRNA against cin85 mRNA

calf intestine phosphatase

Citrine

$\mathrm{Ca}^{2+}$-release-activated channels

chicken serum

Dalton

diacylglycerol

Dulbecco`s modified Eagle`s medium 


\begin{tabular}{|c|c|}
\hline DMSO & dimethylsulfoxide \\
\hline DNA & deoxyribonucleic acid \\
\hline dNTP & deoxynucleoside-triphosphate \\
\hline DTT & 1,4-dithiothreitol \\
\hline E.coli & Escherichia coli \\
\hline ECL & enhanced chemical luminescence \\
\hline EDTA & ethylenediamine tetraacetic acid \\
\hline EGFP & enhanced GFP \\
\hline EGFR & epidermal growth factor receptor \\
\hline EGTA & ethylene glycol tetraacetic acid \\
\hline env & envelope \\
\hline ER & endoplasmic reticulum \\
\hline $\left.\mathrm{F}(\mathrm{ab})^{\prime}\right)_{2}$ & bivalent antigen-binding fragment \\
\hline FACS & fluorescence-activated cell sorter \\
\hline $\mathrm{Fc}$ & fragment crystalline \\
\hline FCS & fetal calf serum \\
\hline FITC & fluorescein-5-isothiocyanate \\
\hline Gads & Grb2-related adaptor protein downstream of SHC \\
\hline gag & gene encoding p55 \\
\hline GFP & Green fluorescent protein \\
\hline Grb2 & Growth factor receptor-bound protein 2 \\
\hline GST & Glutathione-S-transferase \\
\hline $\mathrm{HA}$ & hemagglutinin \\
\hline HEPES & (4-(2-hydroxyethyl)-1-piperazineethanesulfonic acid \\
\hline HisD & histidinol \\
\hline Hpk1 & Hematopoietic progenitor kinase 1 \\
\hline HRPO & Horseradish peroxidase \\
\hline $\mathrm{I}_{\mathrm{CRAC}}$ & $\mathrm{Ca}^{2+}$ release activated $\mathrm{Ca}^{2+}$ current \\
\hline $\lg$ & Immunoglobulin \\
\hline $\lg \mathrm{H}$ & Ig heavy chain \\
\hline $\lg L$ & Ig light chain \\
\hline Indo-1 & $\begin{array}{l}\text { 2-[4-(bis(carboxymethyl)amino)-3-[2-[2-(bis(carboxymethyl)amino)-5- } \\
\text { methylphenoxy]ethoxy]phenyl]-1H-indole-6-carboxylic acid }\end{array}$ \\
\hline IP & immuno purification \\
\hline IP3 & inositol-1, 4, 5-trisphosphate \\
\hline $\mathrm{IP}_{3} \mathrm{R}$ & $\mathrm{IP}_{3}$ receptor \\
\hline IPTG & isopropyl- $\beta$-D-thiogalactopyranoside \\
\hline
\end{tabular}




\begin{tabular}{|c|c|}
\hline ITAM & immunoreceptor tyrosine-based activation motif \\
\hline Itk & IL-2 inducible T cell kinase \\
\hline k & kilo \\
\hline $\mathrm{kb}$ & kilobase \\
\hline LA & left arm \\
\hline Lat & Linker of activated T cells \\
\hline LB & lysogeny broth \\
\hline Lyn & Lck/yes-related novel protein tyrosine kinase \\
\hline M & Mega \\
\hline $\mathrm{m}$ & milli \\
\hline $\mathrm{mAb}$ & monoclonal antibody \\
\hline MFI & mean fluorescence intensity \\
\hline $\mathrm{mlg}$ & membrane-bound immunoglobulin \\
\hline mock $^{\text {sh }}$ & DT40 B cells expressing mock shRNA \\
\hline mRNA & messenger RNA \\
\hline$n$ & nano \\
\hline NFAT & Nuclear factor of activated T cells \\
\hline NFKB & Nuclear factor of $\mathrm{k}$ light polypeptide gene enhancer in B cells \\
\hline NP-40 & nonidet P-40 \\
\hline OD & optical density \\
\hline $\mathrm{p}$ & pico \\
\hline PAGE & polyacrylamide gel electrophoresis \\
\hline PBS & phosphate-buffered saline \\
\hline $\mathrm{PIP}_{2}$ & phosphatidyl-inositol-4, 5-bisphosphate \\
\hline PLCy2 & Phospholipase C-ү2 \\
\hline PLD & Phosholipase D \\
\hline pol & gene encoding protease, reverse transcriptase and integrase \\
\hline PTK & protein tyrosine kinase \\
\hline puro & puromycin resistance \\
\hline pY & phosphotyrosine \\
\hline RA & right arm \\
\hline ref. & reference \\
\hline RNA & ribonucleic acid \\
\hline RNAi & RNA interference \\
\hline RNase & ribonuclease \\
\hline rpm & revolutions per minute \\
\hline RPMI & Roswell Park Memorial Institute \\
\hline
\end{tabular}




\begin{tabular}{|c|c|}
\hline RT & room temperature \\
\hline RTK & receptor tyrosine kinase \\
\hline Ruk & Regulator of ubiquitous kinase \\
\hline SD & standard deviation \\
\hline SDS & sodium dodecylsulfate \\
\hline $\mathrm{SH} 2$ & src homology 2 \\
\hline $\mathrm{SH} 3$ & src homology 3 \\
\hline SLP65 & $\mathrm{SH} 2$ domain-containing leukocyte protein of $65 \mathrm{kDa}$ \\
\hline SOCE & store-operated $\mathrm{Ca}^{2+}$ entry \\
\hline src & rous sarcoma oncogene \\
\hline Ste5 & Sterile 5 \\
\hline STIM1 & Stromal interaction molecule 1 \\
\hline SUMO & small ubiquitin-like modifier \\
\hline Syk & Spleen tyrosine kinase \\
\hline $\mathrm{t}$ & time \\
\hline TAE & Tris acetate EDTA buffer \\
\hline TBE & Tris borate EDTA buffer \\
\hline TBS & Tris-buffered saline \\
\hline TCR & T cell antigen receptor \\
\hline TEMED & $\mathrm{N}, \mathrm{N}, \mathrm{N}^{`}, \mathrm{~N} `$-tetramethylethylene-diamine \\
\hline $\mathrm{TI}$ & T cell-independent \\
\hline TIRAP & Toll-interleukin 1 receptor domain-containing adapter protein \\
\hline TIRF & total internal reflection \\
\hline Tris & Tris-(hydroxymethyl)-aminomethane \\
\hline Triton $\mathrm{X}-100$ & polyethylene glycol p-(1,1,3,3-tetramethylbutyl)-phenyl ether \\
\hline Tween-20 & polyoxyethylene sorbitan monolaurate \\
\hline$U$ & units \\
\hline UTR & untranslated region \\
\hline UV & ultraviolet \\
\hline $\mathrm{v} / \mathrm{v}$ & volume per volume \\
\hline VSV-G & vesicular stomatitis virus glycoprotein \\
\hline$w / v$ & weight per volume \\
\hline wt & wild-type \\
\hline X-Gal & 5-bromo-4-chloro-3-indolyl- beta-D-galactopyranoside \\
\hline
\end{tabular}




\section{Amino acids and Deoxyribonucleotides}

Table 0.1: Amino acids

\begin{tabular}{|c|c|c|}
\hline amino acid & 3-letter code & single-letter code \\
\hline Alanine & Ala & $A$ \\
\hline Cysteine & Cys & C \\
\hline Aspartic acid & Asp & D \\
\hline Glutamic acid & Glu & $E$ \\
\hline Phenylalanine & Phe & $\mathrm{F}$ \\
\hline Glycine & Gly & $\mathrm{G}$ \\
\hline Histidine & His & $\mathrm{H}$ \\
\hline Isoleucine & Ile & I \\
\hline Lysine & Lys & K \\
\hline Leucine & Leu & L \\
\hline Methionine & Met & M \\
\hline Asparagine & Asn & $\mathrm{N}$ \\
\hline Proline & Pro & $P$ \\
\hline Glutamine & Gln & Q \\
\hline Arginine & Arg & $\mathrm{R}$ \\
\hline Serine & Ser & $S$ \\
\hline Threonine & Thr & $\mathrm{T}$ \\
\hline Valine & Val & V \\
\hline Tryptophan & Trp & W \\
\hline Tyrosine & Tyr & Y \\
\hline
\end{tabular}

Table 0.2: Deoxyribonucleotides

\begin{tabular}{cc}
\hline Deoxyribonucleotides & symbols \\
\hline deoxyadenosine monophosphate & $\mathrm{a}, \mathrm{A}$ \\
deoxycytidine monophosphate & $\mathrm{c}, \mathrm{C}$ \\
deoxyguanine monophosphate & $\mathrm{g}, \mathrm{G}$ \\
deoxythymidine monophosphate & $\mathrm{t}, \mathrm{T}$ \\
\hline
\end{tabular}




\section{Introduction}

The systematic, scientific study of immunology started in the late $18^{\text {th }}$ century, when Edward Jenner reported to induce immunity to smallpox in a young boy after inoculating him with a much less virulent pathogen causing cowpox. The principle of vaccination as safeguard against pathogens was proven by Louis Pasteur who was able to generate a vaccine against cholera in chickens (Pasteur 1880). Immunisation of humans with live, attenuated or nonvirulent pathogens or their components is still the underlying principle of today's vaccination (from lat. vacca, cow), which is highly effective in preventing infectious disease in humans.

Emil von Behring and Shibasaburo Kitasato established an antitoxic serum therapy based on the discovery that the serum of animals immunised with tetanus or diphtheria was able to neutralise the bacterial toxins (von Behring and Kitasato 1890). This therapy was assigned as a humoral therapy (from lat. humor, fluid) because the antitoxins were present in the body fluids. With this von Behring anticipated the existence of antibodies (antitoxins) and antibodysecreting cells that are part of the immune system and accomplish the body's humoral immune response. Humoral immune responses together with cell-mediated ones provide adaptive immunity, the power of which is highly specific antigen recognition.

A principle for the generation of antigen-specific antibodies was provided by Paul Ehrlich in his side chain theory (Ehrlich 1900). He proposed that cells display side chains (or receptors) of various specificity on their surface that, if complementary to the antigen, are blocked. This would lead to production of soluble receptors with the very same specificity as the receptor that was engaged. This mechanism holds true today except for the fact that only $B$ cells do express the side chains (B cell antigen receptor) and importantly, each B cell expresses receptors of only one specificity. The origin of the cells that mediate the humoral immune response was identified when the Bursa of Fabricius, a bird-restricted organ, was surgically removed from birds. These animals could not produce antibodies (Glick 1956). Thus, these cells were named $B$ cell (B for Bursa of Fabricius). In mammals the $B$ cells were found to develop in the bone marrow (Mitchell and Miller 1968).

In any given moment plenty of $B$ cells, each of unique receptor specificity, exist in the human body. According to the clonal selection theory that was introduced by Macfarlane Burnet antigen binding results in proliferation of the antigen-specific $B$ cell and production of daughter cells of identical specificity (Burnet 1959). This B cell clone then produces specific antibodies.

The antibodies provide humoral immunity by three ways: Firstly, antibodies prevent pathogens from entering cells by binding to them. This process is called neutralisation. Secondly, opsonisation, the "tagging" of pathogens by antibodies, leads to phagocytosis of 
the pathogen by cells of the innate immune system. Thirdly, antibodies on a pathogen are recognised by the complement components leading to further activation of the complement system. This results in direct lysis of the pathogen or further opsonisation and phagocytosis. The nearly infinite diversity of the $\mathrm{B}$ cell antigen receptors and thus antibodies in recognising antigen is accomplished by rearrangement of the receptor's gene segments. This somatic recombination was discovered by Susumu Tonegawa in the early 1980s (Tonegawa 1983).

\subsection{The B cell antigen receptor}

Most of our knowledge from the structure of B cell antigen receptors (BCRs) comes from investigations of antibodies, which are proteins of the class of globulins, the immunoglobulins $(\mathrm{lg})$, and take the shape of a Y. The membrane-bound form of the $\mathrm{lg}$ molecule $(\mathrm{mlg})$ constitutes most of the extracellular part of the BCR, together with its transmembrane proteins $\lg \alpha(\mathrm{CD} 79 \mathrm{a})$ and $\lg \beta(\mathrm{CD} 79 \mathrm{~b})$ that connect the mlg to intracellular kinases (Campbell and Cambier 1990; Hombach et al. 1990; Wienands et al. 1990).

The mlg consists of four polypeptide chains: two disulfide-linked $\lg$ heavy chains $(\lg \mathrm{H})$ with each linked to one lg light chain ( $\lg \mathrm{L}$ ) by another disulfide bond. Sequence analyses revealed the existence of five different classes of $\lg \mathrm{H}-\alpha, \varepsilon, \delta, \mu$ and $\gamma$ that determine the effector function of the secreted antibody molecule (Venkitaraman et al. 1991; Reth 1992). In contrast, there exist only two different types of $\lg \mathrm{L}-\mathrm{K}$ and $\lambda$, for which a functional difference is not known (Reth 1992). The amino-terminal parts of $\mathrm{lgH}$ and $\mathrm{IgL}$, corresponding to the first Ig domain, displayed high variability in sequence (Wu and Kabat 1970; Johnson and Wu 2000). Unlike these variable domains $\left(V_{H}\right.$ and $V_{L}$, respectively), the remaining domains are constant among $\mathrm{IgL}$ and $\mathrm{IgH}$ of the same isotype (constant domains, $\mathrm{C}_{\mathrm{L}}$ and $\mathrm{C}_{\mathrm{H}}$ ) (Porter 1991). The hypervariable regions (or complementarity-determining regions) in the variable domains are the site of antigen binding as deduced by crystallography of antibody and antigen complexes (Amzel et al. 1974). Due to allelic exclusion the two $\mathrm{lgL}$ and $\mathrm{lgH}$ of one $\mathrm{Ig}$ are identical in sequence resulting in two identical antigen binding sites. Thereby the avidity of an interaction between an $\lg$ molecule and its antigen is increased.

The binding of antigen to the mlg is transduced into the interior of the B cell by the BCR's transmembrane components Iga (CD79a) and Ig $\beta$ (CD79b) (Reth 1992; Sanchez et al. 1993; Reth and Wienands 1997). Ig $\alpha$ and $\lg \beta$ form a disulfide-linked hetero-dimer and are noncovalently associated with the mlg (Campbell and Cambier 1990; Hombach et al. 1990) building a $[\mathrm{lgH}+\operatorname{lgL}]_{2}[\lg \alpha+\lg \beta]_{1}$ multiprotein complex (Schamel and Reth 2000a). The BCR can only be expressed on the surface when fully assembled (Reth 1992; Schamel and Reth 2000b). 
During B cell development the pre BCR is expressed on the surface of pre B cells. It is composed of the two $\lg \mathrm{H}$ coupled to the $\lg \alpha / \beta$ hetero-dimer, but instead of $\lg \mathrm{L}$ it employs $\lambda 5$ and VpreB as surrogate light chains (Tsubata and Reth 1990; Reth 1991). Correct assembly of the pre BCR allows further B cell development and expression of the mature BCR (Melchers et al. 1995). Signalling through the (pre) BCR is prerequisite for B cell quality control that permits on the one hand development, survival and activation of functional, immuno-competent B cells, and on the other hand induces silencing of dysfunctional e.g. auto reactive B cells (King and Monroe 2000; Wang and Clark 2003; Grande et al. 2007).

\subsection{B cell responses to engagement of the BCR}

The natural antigens for B cells are extracellular pathogens and their secreted toxins. Antigen binding to the BCR must be converted into an intracellular signal and it results in $\mathrm{B}$ cell polarisation and internalisation of the antigen-bound BCRs.

In order to induce structural changes in the BCR that activate a B cell, the antigen is believed to be of a multivalent nature, due to the failure of soluble, monovalent antigen to induce BCR signalling. Thus, the cross-linking model evolved in which antigen brings BCRs into close proximity to allow further signal transduction. Cross-linked BCRs then translocate into lipid rafts, which are cholesterol- and sphingolipid-rich microdomains of the plasma membrane, while resting BCRs are found outside of these structures (Cheng et al. 1999; Aman and Ravichandran 2000). According to the lipid raft model this translocation annuls the spatial segregation of BCRs with downstream effector proteins, e.g. kinases (Cheng et al. 1999; Aman and Ravichandran 2000; Sohn et al. 2006; Sohn et al. 2008). In a markedly contrasting model Reth and colleagues propose that antigen binding leads to disruption of BCR oligomers and thereby initiation of signalling (Reth 2001). However, the real nature of the BCR on living B lymphocytes and the initial steps in signal transduction await further elucidation (for review see (Engels et al. 2008)).

After antigen binding $B$ cells respond with morphological changes and polarisation. This is characterised by a cap-like structure of BCR molecules after cross-linking with soluble antigen (Taylor et al. 1971; Schreiner and Unanue 1977). This polarisation is also evident between antigen-loaded antigen presenting cells and antigen-specific BCRs on B cells (Batista et al. 2001). High resolution imaging techniques enabled the spatiotemporal analysis of this B cell polarisation (Fleire et al. 2006): In response to antigen coupled to membranes, $\mathrm{BCR} /$ antigen microclusters are formed that recruit BCR signalling effector proteins (Depoil et al. 2008; Sohn et al. 2008). Their initial formation does not depend on BCR signalling, but is together with cytoskeleton remodelling required to propagate spreading over the antigenloaded membrane (Weber et al. 2008). During spreading more antigen is encountered and 
thereby more microclusters are formed as a function of affinity and density of the membranebound antigen (Fleire et al. 2006; Depoil et al. 2008; Weber et al. 2008). In a subsequent contraction response antigen/BCR microclusters are collected into a central cluster, referred to as central supramolecular activation cluster of the immunological synapse. The B cell's synapse matches the structure of the immunological synapse first discovered in $T$ cells and natural killer cells (Monks et al. 1998; Davis et al. 1999). Whether in vivo BCR activation is mediated via membrane-bound or soluble antigen is currently a matter of debate.

Finally, antigen-bound BCRs are internalised. There is accumulating evidence that antigen/BCR internalisation depends on BCR signalling (Shaw et al. 1990; Song et al. 1995; Stoddart et al. 2002) and employs BCR effector proteins like Casitas B-lineage lymphoma proto-oncogene (Cbl) (Kitaura et al. 2007). BCR internalisation is accomplished by three, partially redundant routes that rely on the actin cytoskeleton, lipid rafts and/or clathrin-coated pits (Salisbury et al. 1980; Guagliardi et al. 1990; Stoddart et al. 2002; Stoddart et al. 2005). After internalisation, the antigen is presented by the B cell on major histocompatability complex II (MHCII) to receive T cell help (Lanzavecchia 1985; Batista et al. 2001). The antigen presented to the cognate $T$ cell is of a peptoid nature and called $T$ cell-dependent antigen. In contrast non-peptide, $\mathrm{T}$ cell-independent $(\mathrm{TI})$ antigens do not induce cognate $\mathrm{T}$ cell help and activate B cells either by secondary receptors ( $\mathrm{TI}-1$ antigens) or heavy crosslinking of BCRs (TI-2 antigens) (Fagarasan and Honjo 2000). Finally, B cells differentiate into plasma cells that secrete antibody or - after $T$ cell help - into memory B cells that comprise long lasting immunity against re-infection.

\subsection{The $\mathrm{BCR}$ signalling cascade leading to a $\mathrm{Ca}^{2+}$ response}

On path of the BCR signal is characterised by an intracellular protein phosphorylation cascade followed by an increase in the intracellular concentration of the second messenger $\mathrm{Ca}^{2+}$. This leads to central cellular responses like proliferation, differentiation or apoptosis of the B cell (Niiro and Clark 2002). Fundamental signalling pathways, like Nuclear factor of $\mathrm{K}$ light polypeptide gene enhancer in $\mathrm{B}$ cells $\left(\mathrm{NF}_{\mathrm{K}} \mathrm{B}\right)$ or Nuclear factor of activated $\mathrm{T}$ cells (NFAT) rely on $\mathrm{Ca}^{2+}$. Hence, maintenance of $\mathrm{Ca}^{2+}$ gradients and $\mathrm{Ca}^{2+}$ concentrations in resting cells as well as $\mathrm{BCR}$-induced dynamic changes of intracellular $\mathrm{Ca}^{2+}$ concentration are central for B cell biology. 


\subsubsection{The BCR-induced protein phosphorylation cascade}

Stimulation of the BCR is transduced by the $\lg \alpha / \beta$ hetero-dimer that contains two copies of the highly conserved immunoreceptor tyrosine-based activation motif (ITAM) (Reth 1989; Cambier 1995). The ITAM serves as tyrosine phosphorylation motif for Src kinases (Lyn, Fyn and Blk) that become activated upon antigen binding to the BCR (Burkhardt et al. 1991; Yamanashi et al. 1991; Campbell and Sefton 1992; Clark et al. 1992; Lin and Justement 1992). Doubly-phosphorylated ITAMs are specifically recognised by the tandemly arranged Src homology 2 (SH2) domains of Syk (Wienands et al. 1995; Fu et al. 1998; Futterer et al. 1998). Binding of Syk to the ITAM results in translocation of Syk from the cytosol to the BCR and increased Syk activity, due to auto phosphorylation and possibly, direct phosphorylation by Src kinases (Kurosaki et al. 1994; Kurosaki et al. 1995; Rowley et al. 1995; Kimura et al. 1996). However, a BCR-induced $\mathrm{Ca}^{2+}$ response can be generated in the absence of SrC kinases, albeit with a strong delay (Takata et al. 1994). The two central kinases Lyn and Syk disperse the incoming signal. One substrate of Syk is SLP65 (Wienands et al. 1998) alternatively named B cell linker protein (BLNK) (Fu et al. 1998) or B cell adaptor containing a SH2 domain protein (BASH) (Goitsuka et al. 1998). Once phosphorylated SLP65 assembles the trimolecular $\mathrm{Ca}^{2+}$ initiation complex comprising Bruton`s tyrosine kinase (Btk), Phospholipase C-y2 (PLCY2) and SLP65 itself. Btk is phosphorylated and thus activated by Lyn or by Syk (Rawlings et al. 1996; Kurosaki and Kurosaki 1997). In cis assembly of Btk and its substrate PLCY2 on one SLP65 molecule, results in phosphorylation and activation of PLCy2 by Btk (Hashimoto et al. 1999; Ishiai et al. 1999a; Ishiai et al. 1999b; Chiu et al. 2002). SLP65 is crucial for membrane localisation of PLCү2 as demonstrated by studies, in which a membrane (Ishiai et al. 1999a) or a lipid raft anchor (Rodriguez et al. 2003) fused to PLCY2 rescued the signalling defect in s/p65 $\mathrm{B}$ cells. Furthermore, membrane association of PLCy2 depends mainly on its C-terminal SH2 domain, with which it binds SLP65 and is reduced in s/p65 $5^{-1} \mathrm{~B}$ cells (Ishiai et al. 1999a). Btk activity was increased in the presence of SLP65 as compared to absence of SLP65 (Baba et al. 2001). Despite a fundamental contribution of SLP65 to the sub cellular navigation of the $\mathrm{Ca}^{2+}$ initiation complex, the pleckstrin homology $(\mathrm{PH})$ domains of Btk and PLCY2 can stabilise their membrane localisation (Bolland et al. 1998; Falasca et al. 1998). At the plasma membrane activated PLCY2 hydrolyses phosphatidyl-inositol-4,5-bisphosphate $\left(\mathrm{PIP}_{2}\right)$ into membrane resident diacylglycerol (DAG) and soluble inositol-1, 4, 5-trisphosphate ( $\left(\mathrm{IP}_{3}\right)$ (Kurosaki et al. 2000). 


\subsubsection{The two phases of the BCR-triggered $\mathrm{Ca}^{2+}$ response}

Generation of $\mathrm{IP}_{3}$ results in a $\mathrm{Ca}^{2+}$ response that is characterised by two phases: a fast and transient release of $\mathrm{Ca}^{2+}$ from the endoplasmic reticulum (ER) lumen followed by a second phase which is more sustained and characterised by the influx of $\mathrm{Ca}^{2+}$ across the plasma membrane.

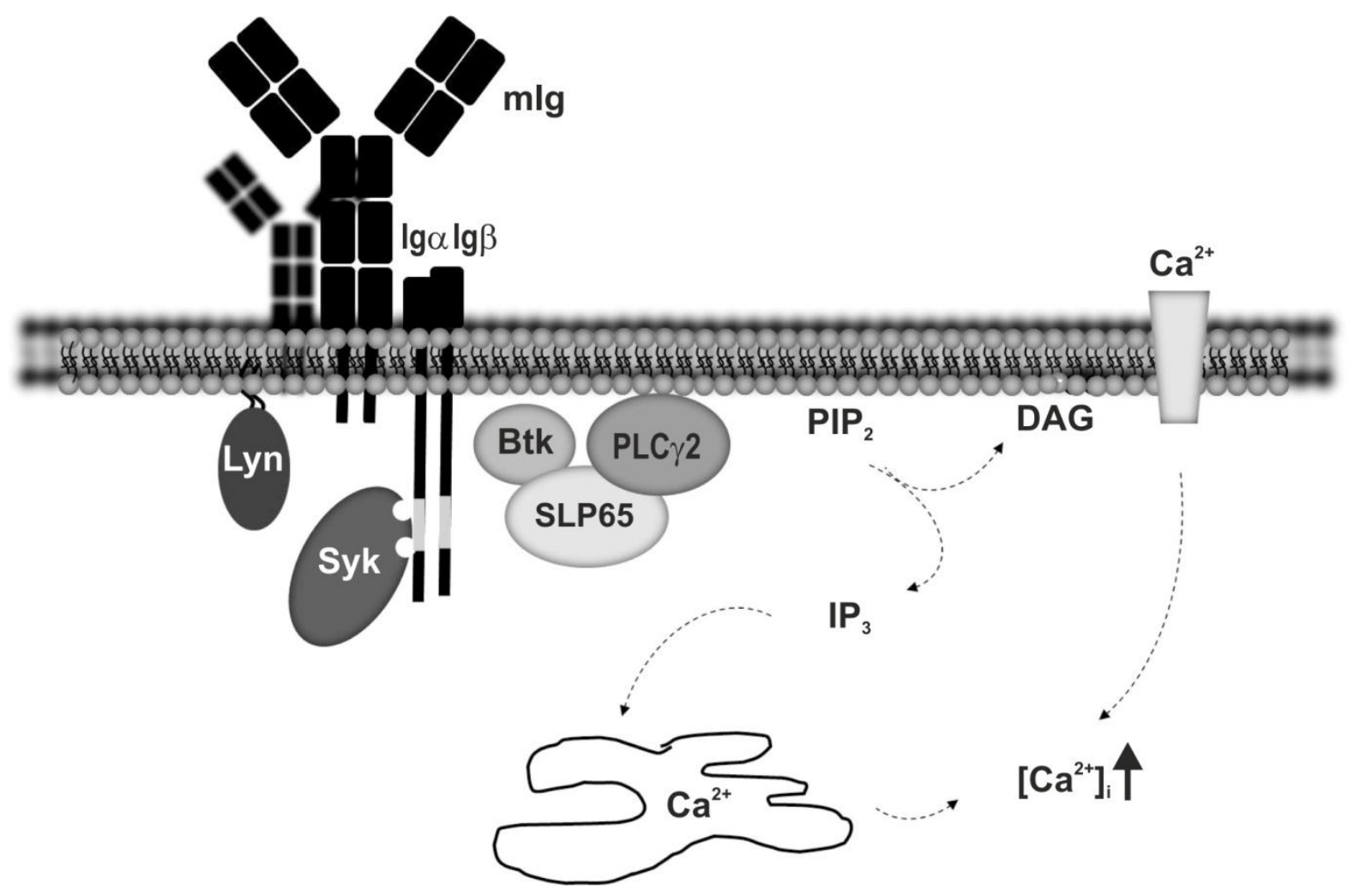

Figure 1.1: $\mathrm{BCR}$-induced signalling events leading to a rise in intracellular $\mathrm{Ca}^{2+}$ concentration. After binding of antigen (not shown), BCRs are clustered on the B cell surface. Subsequent activation of Lyn leads to phosphorylation of the ITAMs in the Iga/ $\beta$ hetero-dimer followed by translocation and activation of Syk to the phosphorylated ITAMs. Active Syk phosphorylates its substrate SLP65. Phosphorylated SLP65 allows the formation and translocation of the $\mathrm{Ca}^{2+}$ initiation complex comprising SLP65, Btk and PLCy2 to the plasma membrane. In the $\mathrm{Ca}^{2+}$ initiation complex, activated Btk phosphorylates PLCY2, which then hydrolyses $\mathrm{PIP}_{2}$ into DAG and $\mathrm{IP}_{3}$. Enhanced $\mathrm{IP}_{3}$ levels lead to a release of $\mathrm{Ca}^{2+}$ from the $\mathrm{ER}$ and subsequently across the plasma membrane. Changes in the intracellular $\mathrm{Ca}^{2+}$ concentration $\left(\left[\mathrm{Ca}^{2+}\right]_{\mathrm{i}}\right)$ result in transcriptional changes and differential $\mathrm{B}$ cell outcomes. For review see (Engelke et al. 2007).

$I P_{3}$ binding to the $\mathrm{IP}_{3} \mathrm{R}$ leads to a release of $\mathrm{Ca}^{2+}$ from the ER stores into the cytosol along a $\mathrm{Ca}^{2+}$ concentration gradient (Patterson et al. 2004). $I \mathrm{P}_{3} \mathrm{R}$ are ligand-gated $\mathrm{Ca}^{2+}$ channels and deletion of all three $\mathrm{IP}_{3} \mathrm{R}$ isoforms results in complete abrogation of $\mathrm{Ca}^{2+}$ mobilisation in DT40 $B$ cells (Sugawara et al. 1997). The decreased $\mathrm{Ca}^{2+}$ concentration in the ER lumen is sensed by the ER transmembrane protein Stromal interaction molecule 1 (STIM1) (Roos et al. 2005; Zhang et al. 2005; Baba et al. 2006). Aggregated STIM1 molecules move towards the 
plasma membrane to activate $\mathrm{Ca}^{2+}$-release-activated channels (CRAC) (Zhang et al. 2005; Baba et al. 2006; Huang et al. 2006; Luik et al. 2006; Xu et al. 2006). The CRACs allow influx of $\mathrm{Ca}^{2+}$ from the extracellular space into the cell's interior, a mechanism called storeoperated $\mathrm{Ca}^{2+}$ entry (SOCE). ORAI, a four transmembrane spanning protein, is a component of CRAC (Feske et al. 2006; Prakriya et al. 2006; Vig et al. 2006) and important in generating a CRAC current ( $\left.I_{C R A C}\right)$ that operates in immune cells (Hoth and Penner 1992). The BCRinduced $\mathrm{Ca}^{2+}$ response strongly depends on the developmental stage of the $\mathrm{B}$ cell (Hoek et al. 2006) and engagement of activatory and inhibitory coreceptors. These together with the nature of the antigen determine the amplitude and duration of the BCR-induced $\mathrm{Ca}^{2+}$ signal which are critical determinants for the transcriptional changes leading to appropriate $B$ cell outcomes (Dolmetsch et al. 1997; Engelke et al. 2007). Figure 1.1 gives a simplified overview of the described BCR-induced events leading to an increase in intracellular $\mathrm{Ca}^{2+}$ concentration.

\subsection{The adaptor protein SLP65 and its steady ligands CIN85 and CD2AP}

Protein complexes are assumed to be the executive modules of cellular functions instead of single proteins (Gavin et al. 2002; Gavin et al. 2006). Adaptor proteins exert an important role in formation of multimolecular complexes assembling two or more ligands by virtue of their protein interaction domains (Pawson and Nash 2003). Thereby, they can coordinate different enzymatic functions, separate distinct signalling pathways or increase or lower thresholds of cellular activation (Burack et al. 2002). Moreover, adaptors function in spatial organisation of protein/protein complexes by anchoring or targeting them to sub cellular areas, as does SLP65 in the $\mathrm{Ca}^{2+}$ initiation complex. To identify an interaction partner of SLP65 enabling its membrane localisation, our group in collaboration with the group of Prof. Dr. H. Urlaub established a mass spectrometric approach (Neumann et al. 2009; Oellerich et al. 2009; Oellerich et al. 2011). Analysis of the SLP65 interactome revealed, among others, a steady interaction of SLP65 with CD2-associated protein (CD2AP) and Cbl-interacting protein of $85 \mathrm{kDa}(\mathrm{CIN} 85)$.

\subsubsection{Structure and function of SLP65}

SLP65 was identified on the basis that it is heavily phosphorylated upon BCR activation and is expressed only in B cells and macrophages (Fu et al. 1998; Goitsuka et al. 1998; Wienands et al. 1998; Bonilla et al. 2000). SLP65 is an adaptor protein comprising an Nterminal basic effector domain (BED) (Hermann 2009) or leucin-zipper (Kohler et al. 2005), five consensus tyrosine phosphorylation sites, several proline-rich motifs and a C-terminal 
SH2 domain. Among the proline-rich motifs human SLP65 harbours three atypical prolinearginine motifs with a PxxxPR consensus sequence (single-letter amino acid code, $x$ denotes any residue): PPSVPR $_{49}$, PSPLPR $_{248}$ and PIPLPR PI13 $_{3}$ (see figure 1.2). SLP65 interacts with PLCy2, Btk, Guanine nucleotide exchange factor (Vav), Non catalytic region of tyrosine kinase (Nck) in a phosphorylation-dependent manner (Fu et al. 1998; Wienands et al. 1998; Hashimoto et al. 1999; Ishiai et al. 1999b; Su et al. 1999; Chiu et al. 2002). Growth factor receptor-bound protein 2 (Grb2) binds constitutively to SLP65 via its C-terminal src homology 3 (SH3) domain and binding is increased after phosphorylation of SLP65 (Fu et al. 1998; Wienands et al. 1998; Grabbe and Wienands 2006). Hematopoietic progenitor kinase 1 (Hpk1), Syk and Iga are ligands for the SH2 domain of SLP65 (Engels et al. 2001; Sauer et al. 2001; Kabak et al. 2002; Kulathu et al. 2008). SLP65-deficiency in mice leads to a block in B cell development at the large pre B cell stage (Jumaa et al. 1999; Minegishi et al. 1999; Pappu et al. 1999; Hayashi et al. 2000; Xu et al. 2000). However, the developmental block in slp65 mice is not complete since immature B cells can be detected possibly due to expression of compensatory adaptors (Su and Jumaa 2003). These s/p65 ${ }^{-1}$ immature B cells show impaired $\mathrm{BCR}$-induced $\mathrm{Ca}^{2+}$ mobilisation and proliferation. In humans SLP65deficiency resulted in a complete block of B cell development (Minegishi et al. 1999) and was associated with acute lymphoblastic leukaemia in some of the patients (Jumaa et al. 2003; Imai et al. 2004). Ablation of SLP65 expression in DT40 B cells results in a complete absence of BCR-triggered $\mathrm{Ca}^{2+}$ flux (Ishiai et al. 1999a).

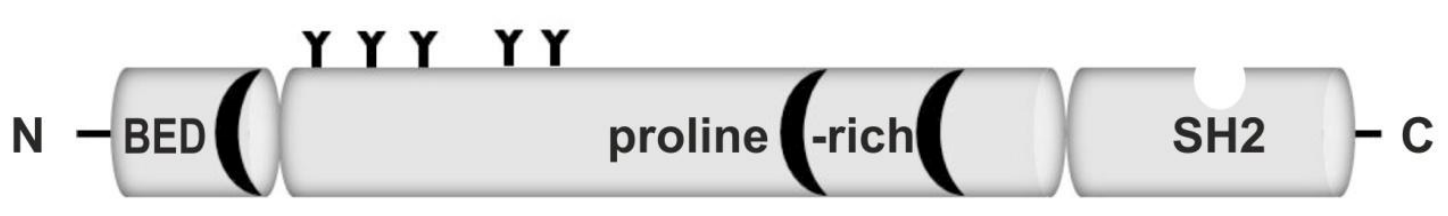

Figure 1.2: SLP65 domain architecture modified from (Koretzky et al. 2006).

The first 50 amino acids of SLP65 comprise a basic effector domain (BED) which is characterised by an accumulation of basic amino acids. This part is followed by 5 consensus tyrosine phosphorylation sites $(\mathbf{Y})$ and proline-rich motifs. Among these proline-rich motifs are three of the PxxxPR type $(\mathbf{(})$. In its C-terminus SLP65 harbours an SH2 domain (for review see (Koretzky et al. 2006)).

\subsubsection{Membrane recruitment of SLP adaptors}

SLP65 membrane localisation depends on its N-terminus (Kohler et al. 2005) and C-terminal SH2 domain (Abudula et al. 2007). A ligand for the SLP65 N-terminus is not known, but it could afford electrostatic interactions with phospholipids (Hermann 2009). Truncation of the SH2 domain of SLP65 resulted in decreased SLP65 phosphorylation, membrane recruitment 
and $\mathrm{Ca}^{2+}$ mobilisation in response to BCR stimulation (Abudula et al. 2007). The SH2 domain binds inducibly to the non-ITAM phospho-tyrosine in the Iga cytoplasmic domain (Engels et al. 2001; Kabak et al. 2002), but exchange of the Iga non-ITAM tyrosine has only marginal effect on $\mathrm{Ca}^{2+}$ mobilisation and thus, is at least not the only membrane anchor for SLP65 (Kabak et al. 2002; Patterson et al. 2006).

In T cells, membrane recruitment of SLP76, the T cell paralogue of SLP65 is mediated by a transmembrane adaptor protein called Linker of activated T cells (LAT). LAT becomes phosphorylated after TCR engagement (Brdicka et al. 1998; Zhang et al. 1998). Phosphorylated LAT provides binding sites for Grb2-related adaptor protein downstream of Shc (Gads) which is a constitutive ligand of SLP76 (Liu et al. 1999; Liu et al. 2003). Membrane recruitment of this preformed Gads/SLP76 module leads to phosphorylation of SLP76 at the plasma membrane (Bubeck Wardenburg et al. 1998). In analogy to Btk that phosphorylates PLCY2 in B cells, IL-2 inducible T cell kinase (Itk) binds SLP76 via its SH2 domain and activates LAT associated PLCY1 in T cells (Bunnell et al. 2001). While the membrane anchoring mechanism for SLP76 is clarified, the mechanistic of SLP65 membrane recruitment, including the relevant ligands for the $\mathrm{N}$-terminus or the $\mathrm{SH} 2$ domain of SLP65 have yet to be elucidated.

\subsubsection{The scaffolds CIN85 and CD2AP}

CIN85 and CD2AP are scaffolds constituting their own CIN85/CMS family of adaptor proteins (Dikic 2002). CIN85 and CD2AP have been shown to constitutively or inducibly bind to many proteins involved in receptor tyrosine kinase (RTK) signal transduction pathways, $\mathrm{T}$ cell signalling and receptor internalisation (Dikic 2002). CIN85 (Take et al. 2000), alternatively called Regulator of ubiquitous kinase (Ruk) (Gout et al. 2000), Src-homology 3 encoding, expressed in tumorigenic astrocytes (SETA) (Borinstein et al. 2000) or SH3 domain kinase binding protein 1 (SH3KBP1) (Narita et al. 2001) were cloned from human, rat (Ruk and SETA) and mouse cells, respectively. The cin85 gene displays a complex organisation involving 5 promotors with differing tissue specificity (Buchman et al. 2002) giving rise to up to 8 potential protein isoforms (Finniss et al. 2004). In human T cells two CIN85 isoforms

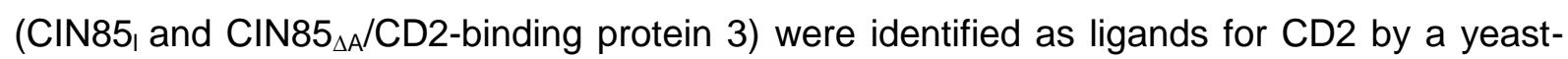
two-hybrid screen (Tibaldi and Reinherz 2003). CIN85 has the same domain architecture as its family member CD2AP (Dustin et al. 1998) also called Cas ligand with multiple SH3 domains (CMS) (Kirsch et al. 1999) or Mesenchyme-to-epithelium transition protein with SH3 domains (Mets-1) (Lehtonen et al. 2000) and both share 54\% similarity on the amino acid level (Dikic 2002). The N-terminal halves of CIN85 and CD2AP are built of three SH3 domains, followed by proline-rich motifs and a C-terminal coiled coil domain (see figure 1.3). Throughout the protein potential serine/threonine phosphorylation sites are evident as well as 
FxDxF sequences that could mediate interaction with the clathrin adaptor protein AP2 (Dikic 2002). Unlike CIN85, CD2AP contains four actin binding sites in its C-terminal half (Kirsch et al. 1999; Tibaldi and Reinherz 2003). CIN85 and CD2AP can form hetero/homo-oligomers via their coiled coil (CC) domains (Kirsch et al. 1999; Borinstein et al. 2000; Watanabe et al. 2000). The first SH3 domain of CIN85 can mediate an intramolecular interaction with a polyproline-peptide in CIN85 (Kowanetz et al. 2003; Tibaldi and Reinherz 2003).

SH3 domains were first identified in Src kinases, the Crk adaptor protein and in PLCY1 and are 60 amino acids in size (Mayer et al. 1988; Stahl et al. 1988). In their tertiary structure $\mathrm{SH} 3$ domains display three grooves that mediate the interaction to the two prolines in the xФРxФPx consensus motif ( $\Phi$ denotes hydrophobic amino acids) (Mayer 2001). The third groove usually mediates binding to a basic amino acid either $\mathrm{N}$ - or C-terminal of the xФРxФPx motif (Feng et al. 1994; Lim et al. 1994; Mayer and Eck 1995). In general SH3 domains have modest specificity at best, but residues outside the core motif can contribute to specificity (Feng et al. 1995). The SH3 domains of CIN85 and CD2AP have a recognition consensus sequence that differs from the classical $\times Ф P \times Ф P x$ motif. They recognise an atypical proline-arginine motif with the consensus sequence PxxPR, while PxPxPR has a higher affinity (Kowanetz et al. 2003).

In B cells CIN85 was found to interact with Grb2, Son of sevenless 1 (Sos1), the p85 subunit of Phosphatidylinositol-3-kinase (PI3K), c-Cbl and SLP65, but no functional relevance was reported in this study (Watanabe et al. 2000). The interaction of SLP65 with CIN85 is mediated via three atypical proline-arginine motifs in SLP65 and can be abolished upon an amino acid exchange of the arginine in the last position to an alanine (Kurakin et al. 2003). The interaction of CIN85/CD2AP and the E3 ligase Cbl is also mediated via atypical prolinearginine motifs and increases with phosphorylation of $\mathrm{Cbl}$ after Epidermal growth factor receptor (EGFR) stimulation (Take et al. 2000; Soubeyran et al. 2002). CIN85 is constitutively bound by endophilin, which is involved in vesicle formation, and is recruited to RTK-Cbl complexes after receptor stimulation. Thereby, CIN85 mediates internalisation of RTKs (Petrelli et al. 2002; Soubeyran et al. 2002; Szymkiewicz et al. 2002; Kobayashi et al. 2004). Overexpression of CIN85 in mast cells was shown to accelerate internalisation of the non-RTK receptor for lgE, FceRI (Molfetta et al. 2005).

In T cells, CD2AP and CIN85 interact with the T cell transmembrane protein cluster of differentiation 2 (CD2), which is enhanced after TCR engagement (Dustin et al. 1998; Tibaldi and Reinherz 2003). Truncation of the intracellular part of CD2 or overexpression of a dominant-negative CD2AP variant led to disturbed CD2 clustering (Dustin et al. 1998). Generation of $c d 2 a p \%$ mice supported the function of CD2AP in TCR clustering into the immunological synapse upon contact with antigen loaded APCs (Lee et al. 2003). In contrast after settling cd2ap $^{\%} \mathrm{~T}$ cells on planar lipid bilayers a TCR synapse could be formed. The 
authors discussed that the importance of CD2AP is negligible in conditions when the reorganisation of receptors is possibly easier than in a direct cell to cell contact. Stimulation of $c d 2 a p^{\%} \mathrm{~T}$ cells resulted in delayed, but prolonged phosphorylation of the tyrosine kinase Zeta chain associated protein kinase (Zap70) and $\mathrm{Ca}^{2+}$ flux. This was connected with a failure of TCR degradation, but not internalisation in cd2ap ${ }^{-1}$ T cells (Lee et al. 2003). B lymphocytes were not analysed in this study. The possibly most important role of CD2AP is the maintenance of the kidney architecture. This is demonstrated by CD2AP-deficient mice that die at 6 weeks of age due to a strong nephrotic defect (Shih et al. 1999). A kidney disease, focal segmental glomerulosclerosis, was also reported in humans carrying a homozygous cd2ap mutation (Lowik et al. 2007).

CIN85

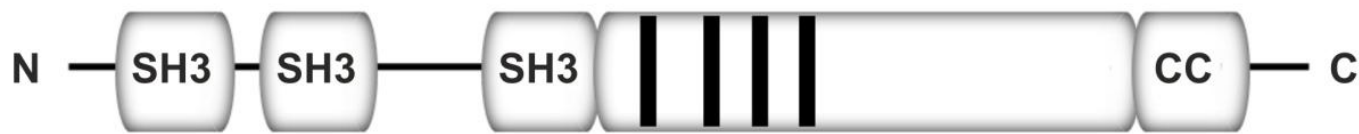

CD2AP

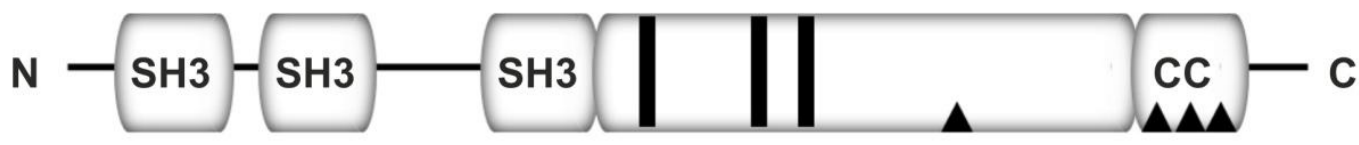

Figure 1.3: Domain architecture of CIN85 and CD2AP modified from (Tibaldi and Reinherz 2003).

CIN85 and CD2AP share 54\% similarity in the amino acid sequence and have a nearly identical domain structure. The $\mathrm{N}$-terminal half of each protein harbours three SH3 domains, followed by proline-rich sequences (black bars) and a C-terminal coiled coil (CC) domain. CD2AP contains 4 actin binding motifs (black triangles), which are lacking in CIN85 (for review see (Dikic 2002)).

The importance of CIN85 and/or CD2AP (CIN85/CD2AP) in constantly binding SLP65 was demonstrated by T.Oellerich in our group: Upon disruption of the CIN85 and CD2AP binding sites in SLP65 a Ca ${ }^{2+}$ mobilisation defect in BCR-stimulated s/p65 $5^{-1}$ DT40 and primary B cells that expressed this SLP65 variant was observed (T.Oellerich, Dr.M.Engelke, Dr.K. Dittmann). Moreover, without being preformed with CIN85 and CD2AP, SLP65 phosphorylation was reduced, while the activation of the upstream kinases Lyn and Syk was not altered (Oellerich et al. 2011). In line with this, our group described earlier that the phosphorylation of SLP65 and other BCR effector proteins needs the BCR as organiser of protein complexes already formed in unstimulated B cells (Wienands et al. 1996). Thus, a preformed transducer complex as prerequisite for a rapid and coordinated BCR signal initiation was assumed. 


\subsection{Aims of the work}

The goal of this thesis was to elucidate the role of CIN85 and CD2AP in BCR signal transduction with special emphasis on their interaction with SLP65:

\section{1) The preformed complex comprising SLP65 and CIN85/CD2AP}

SLP65 has been shown to depend on the interaction with CIN85/CD2AP for generating a full BCR-induced $\mathrm{Ca}^{2+}$ response. Are CIN85 and CD2AP important players in BCR-induced $\mathrm{Ca}^{2+}$ mobilisation, as the SLP65 data suggests, and thus could SLP65 and CIN85/CD2AP constitute a preformed signal transducer element? To approach this, a CD2AP-deficient DT40 B cell line and RNA interference (RNAi) assay are established. The genetically engineered cells are then used for reconstitution experiments and analysed for $\mathrm{Ca}^{2+}$ mobilisation after BCR stimulation. The DT40 B cell line is an appropriate tool for studying BCR signalling mechanisms since many knock-out cell lines already exist and new ones can quite easily be generated.

In order to find out about the binding mode of SLP65, CIN85 and/or CD2AP, their interaction is characterised by biochemical means in DT40 B cell mutants expressing different variants of SLP65, CIN85 or CD2AP.

\section{2) Sub cellular localisation of CIN85 and CD2AP}

Most early BCR signalling effector proteins translocate from the cytosol to the plasma membrane once the BCR is engaged. Where are CIN85 and CD2AP located in resting B cells, and do they react with sub cellular translocation to BCR stimulation? To address this question DT40 B cells expressing fluorescently-tagged CIN85 and CD2AP fusion proteins are analysed by live cell confocal microscopy.

\section{3) CIN85, CD2AP and the BCR}

Previous studies have shown that CD2AP and CIN85 are closely associated with surface receptors; i.e. with $\mathrm{CD} 2$ in the $\mathrm{T}$ cell immunological synapse and with the EGFR for its internalisation. Do CIN85 and CD2AP exert similar functions in the formation of BCR clusters or in BCR internalisation? To test this, firstly, fluorescent versions of CD2AP and CIN85 are analysed for their colocalisation in BCR-containing microclusters by total internal reflection microscopy in collaboration with the group of Dr. Facundo Batista (Lymphocyte Interaction Laboratory, Cancer Research UK, London, UK). Secondly, CD2AP-deficient or CIN85 hypomorphic cells are analysed in a BCR internalisation assays. 


\section{Materials and Methods}

\subsection{Materials}

\subsubsection{Chemicals and reagents}

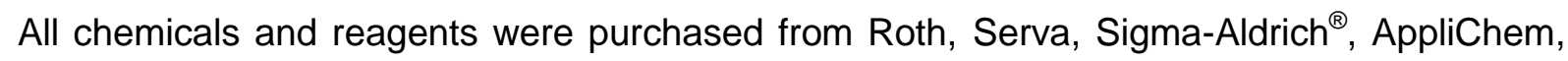
Merck, Invitrogen ${ }^{\mathrm{TM}}$, InvivoGen, Becton Dickinson or Amersham BioSciences in pro analysis quality, unless indicated otherwise.

\subsubsection{Solutions, buffers and media}

Solutions, buffers and media used in this thesis are listed in the corresponding method sections. All buffers and solution were aqueous solutions and stored at room temperature unless indicated otherwise. Solutions were autoclaved at $125^{\circ} \mathrm{C}$ for $30 \mathrm{~min}$.

The following common buffers were used:

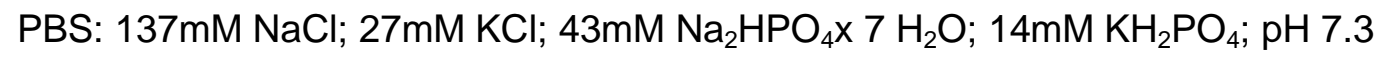

TBS-T: 20mM Tris/HCl pH 7.6; 137mM NaCl; 0.1\% (v/v) Tween-20

\subsubsection{Commercial-Kits}

$\begin{array}{ll}\text { Invisorb }^{\circledR} \text { Spin Plasmid Mini Two Kit } & \text { Invitek } \\ \text { Protino }^{\circledR} \text { Ni IDA } 1000 & \text { Macherey-Nagel } \\ \text { TOPO TA cloning® Kit } & \text { Invitrogen }^{\text {TM }} \\ \text { Wizard }^{\circledR} \text { Plus SV Midiprep Kit } & \text { Promega } \\ \text { Wizard }^{\circledR} \text { SV Gel and PCR Clean-Up System } & \text { Promega }\end{array}$

\subsubsection{Antibodies}

\subsubsection{1 primary antibodies}

All primary antibodies were used in a 1:250 - 1:3,000 dilution in TBS-T with 5\% (w/v) BSA and $0.1 \%(\mathrm{v} / \mathrm{v}) \mathrm{NaN}_{3}$. Application of the antibody is indicated as WB (western blot), IP (immuno purification), $\mathrm{F}$ (flow cytometry) or $\mathrm{S}$ (stimulation). Applied concentrations of the antibodies are indicated in the respective method sections or figure legends. 


\begin{tabular}{|c|c|c|}
\hline Anti-SLP65, mouse monoclonal lgG1 (2C9) & GeneTex, Inc. & WB \\
\hline Anti-Syk, rabbit polyclonal (N19) & Santa Cruz Biotechnologie & WB \\
\hline Anti-CIN85, rabbit polyclonal (C6115) & Sigma-Aldrich $^{\circledR}$ & WB \\
\hline Anti-HA, rat polyclonal & Roche & WB \\
\hline Anti-GFP, mouse monoclonal lgG1 & Roche & WB, IP \\
\hline Anti-GST, rabbit polyclonal & Molecular Probes & WB \\
\hline Anti-actin, rabbit polyclonal & Sigma-Aldrich $^{\circledR}$ & WB \\
\hline Anti-Mouse IgM ( $\mu$ chain specific), goat lgG & SouthernBiotech & IP \\
\hline Anti-chicken IgM, goat lgG & Bethyl Laboratories, Inc. & IP \\
\hline $\begin{array}{l}\text { Cy }{ }^{\mathrm{TM}} 5 \text {-conjugated AffiniPure } \\
\mathrm{F}(\mathrm{ab})_{2} \text { Fragment Goat Anti-Mouse IgM, } \\
\mu \text { Chain Specific }\end{array}$ & Jackson ImmunoResearch & $\mathrm{F}$ \\
\hline Anti-chicken IgM, mouse IgM (M4) & Biozol & $\mathrm{S}$ \\
\hline
\end{tabular}

\subsubsection{2 secondary antibodies}

All secondary antibodies were used in a 1:10,000 dilution in TBS-T.

Immunopure ${ }^{\circledR}$ Goat Anti-Mouse lgG, $(\mathrm{H}+\mathrm{L})$,

Horseradish Peroxidase Conjugated Thermo Scientific

Immunopure ${ }^{\circledR}$ Goat Anti-Rabbit lgG, $(\mathrm{H}+\mathrm{L})$,

Horseradish Peroxidase Conjugated Thermo Scientific

Goat anti-rat $\lg \mathrm{G}(\mathrm{H}+\mathrm{L})$,

Horseradish Peroxidase Conjugated Pierce

Donkey anti-goat $\lg G(\mathrm{H}+\mathrm{L})$,

Horseradish Peroxidase Conjugated Jackson ImmunoResearch

\subsubsection{Bacteria}

One Shot ${ }^{\circledR}$ Top10F' chemo-competent E.coli Invitrogen ${ }^{\mathrm{TM}}$

One Shot ${ }^{\circledR} \mathrm{BL} 21(\mathrm{DE} 3)$ chemo-competent E.coli Invitrogen ${ }^{\mathrm{TM}}$ 


\subsubsection{Enzymes}

Calf intestine phosphatase

Phusion ${ }^{\circledR}$ High-Fidelity DNA Polymerase

Proteinase $\mathrm{K}$

Restriction endonucleases

T4 DNA ligase

Taq PCR Master Mix Kit
New England Biolabs ${ }^{\circledR}$

FINNZYMES (Thermo Fisher Scientific)

Promega

New England Biolabs ${ }^{\circledR}$

New England Biolabs ${ }^{\circledR}$

Qiagen

\subsubsection{Oligonucleotides}

DNA oligonucleotides were synthesised by MWG-Biotech as salt free, lyophilised samples.

Table 2.1: Oligonucleotides

\begin{tabular}{|c|c|c|}
\hline oligonucleotide & sequence $\left(5^{\prime} \rightarrow 3^{\prime}\right)$ & application \\
\hline \multirow[t]{2}{*}{ 5`BamHI-CD2AP } & AAAGGATCCACCATGGTTGACTATATTG & cloning of human \\
\hline & TGGAGTATGAC & cd2ap cDNA \\
\hline \multirow[t]{2}{*}{$3^{\prime}$ Xhol-CD2AP } & AAAGTCGACTCAAGAAGACAGGACAGCT & cloning of human \\
\hline & TTT & cd2ap cDNA \\
\hline \multirow{2}{*}{ 5`BamHI-CIN85 } & AAAGGATCCACCATGGTGGAGGCCATA & cloning of human \\
\hline & GTG & $\operatorname{cin} 85 \mathrm{cDNA}$ \\
\hline \multirow[t]{2}{*}{ 3’Xhol-CIN85 } & GGACTCGAGTCATTTTGATTGTAGAGCT & cloning of human \\
\hline & TTCT & $\operatorname{cin} 85 \mathrm{cDNA}$ \\
\hline CD2APdeltaSH3 & ATAGGATCCACCATGCTTGATAAAGACT & cloning of human \\
\hline fwd & TTCCAAAACCA & $c d 2 a p \Delta \operatorname{sh} 3 \mathrm{cDNA}$ \\
\hline CD2APdeltaSH3 & AAAGTCGACTCAAGAAGACAGGACAGCT & cloning of human \\
\hline -REV & TTT & $c d 2 a p \Delta \operatorname{sh} 3 \mathrm{cDNA}$ \\
\hline CD2APdeltaCC & AAAGGATCCACCATGGTTGACTATATTG & cloning of human \\
\hline fwd & TGGAGTATGAC & $c d 2 a p \Delta c c$ cDNA \\
\hline CD2APdeltaCC & TTTGTCGACTCACACAGATGGCTTTGGA & cloning of human \\
\hline rev & GA & $c d 2 a p \Delta c c$ cDNA \\
\hline
\end{tabular}




\begin{tabular}{|c|c|c|}
\hline $\begin{array}{l}\text { CIN85deltaSH3 } \\
\text { fwd }\end{array}$ & $\begin{array}{l}\text { AAAGGATCCACCATGGACTTTGAAAAGG } \\
\text { AAGGGAATAGA }\end{array}$ & $\begin{array}{l}\text { cloning of human } \\
\operatorname{cin} 85 \Delta \operatorname{sh} 3 \mathrm{cDNA}\end{array}$ \\
\hline CIN85deltaSH3 & GGACTCGAGTCATTTTGATTGTAGAGCT & cloning of human \\
\hline rev & TTCT & $\operatorname{cin} 85 \Delta \operatorname{sh} 3 \mathrm{cDNA}$ \\
\hline $\begin{array}{l}\text { CIN85deltaCC } \\
\text { fwd }\end{array}$ & $\begin{array}{l}\text { AAAGGATCCACCATGGTGGAGGCCATA } \\
\text { GTG }\end{array}$ & $\begin{array}{l}\text { cloning of human } \\
\operatorname{cin} 85 \Delta c c \text { cDNA }\end{array}$ \\
\hline $\begin{array}{l}\text { CIN85deltaCC } \\
\text { _rev }\end{array}$ & $\begin{array}{l}\text { GTTCTCGAGTCACATCTTTGGTTTTCCTT } \\
\text { CCGT }\end{array}$ & $\begin{array}{l}\text { cloning of human } \\
\operatorname{cin} 85 \Delta c c \text { cDNA }\end{array}$ \\
\hline $\begin{array}{l}\text { CD2AP3SH3 } \\
\text { rev }\end{array}$ & $\begin{array}{l}\text { AAAGTCGACTCATTCATTTATCTGGACA } \\
\text { GC }\end{array}$ & $\begin{array}{l}\text { cloning of human } \\
\text { cd2ap3sh3 cDNA }\end{array}$ \\
\hline CITBamBgIN+1 & $\begin{array}{l}\text { TAATAGATCTTACGAATTCCTTGTACAGC } \\
\text { TCGTC }\end{array}$ & $\begin{array}{r}\text { cloning of pMSCV puro } \\
\text { Citrine N-terminal }\end{array}$ \\
\hline CIN85_3SH3rev & $\begin{array}{l}\text { GGACTCGAGTCACGGTGGAAGTAACTT } \\
\text { CACGAA }\end{array}$ & $\begin{array}{l}\text { cloning of human } \\
\text { cin853sh3 cDNA }\end{array}$ \\
\hline chCD2APfwd & $\begin{array}{l}\text { AAAGGATCCACCATGGTGGAGTATATTG } \\
\text { TGGAG }\end{array}$ & $\begin{array}{r}\text { cloning of chicken } \\
\text { cd2ap cDNA }\end{array}$ \\
\hline chCD2APrev & $\begin{array}{l}\text { AAAGTCGACTCATGTAGACATCACTGCT } \\
\text { TT }\end{array}$ & $\begin{array}{r}\text { cloning of chicken } \\
\text { cd2ap cDNA }\end{array}$ \\
\hline $\begin{array}{l}\text { ch_CD2AP } \\
\text { _ablongfwd }\end{array}$ & $\begin{array}{l}\text { ATAGGATCCCCTCCAGTTAAAAATCCAG } \\
\text { CT }\end{array}$ & $\begin{array}{l}\text { cloning of chicken } \\
\text { cd2ap (epitope) }\end{array}$ \\
\hline MOCKsiRNA & $\begin{array}{l}\text { TGCTGTTGACAGTGAGCGATCTCGCTTG } \\
\text { GGCGAGAGTAAGTAGTGAAGCCACAGA } \\
\text { TGTACTTACTCTCGCCCAAGCGAGAGTG } \\
\text { CCTACTGCCTCGGA }\end{array}$ & $\begin{array}{r}\text { cloning of unspecific } \\
\text { shRNA }\end{array}$ \\
\hline cCIN85shRNA & TGCTGTTGACAGTGAGCGCTGACTGAG & cloning of anti-chicken \\
\hline 2389 & $\begin{array}{l}\text { ACTCAAATTTATGTAGTGAAGCCACAGA } \\
\text { TGTACATAAATTTGAGTCTCAGTCATTGC } \\
\text { CTACTGCCTCGGA }\end{array}$ & $\operatorname{cin} 85$ shRNA \\
\hline
\end{tabular}

hS65dBED Bgl CACCAGATCTCCATATGCGAAGGGACTA cloning of human fwd CGCTTCAG s/p65 $\Delta N$-terminus cDNA 


\begin{tabular}{|c|c|c|}
\hline hS65stopnotas & TAATGCGGCCGCTTATGAAACTTTAACT & cloning of human \\
\hline & GCATACTTC & slp65 6 N-terminus cDNA \\
\hline hTIRAP_BamHI & ATAGGATCCTTTGGGGAGTTGGCCTCTT & cloning of human tirap \\
\hline rev & & cDNA \\
\hline hTIRAP_bglfwd & CACCAGATCTCATGGCATCATCGACCTC & cloning of human tirap \\
\hline & $\mathrm{CC}$ & cDNA \\
\hline $\begin{array}{l}\text { CD2APLA } \\
\text { screen }\end{array}$ & GCTGATGTTTGTTCACTGCAATTTGT & $\begin{array}{l}\text { screening of chicken } \\
\text { cd2ap genotype }\end{array}$ \\
\hline CD2APRA & AАСАAАСАССАААТССТСАСТТGСА & screening of chicken \\
\hline screen & & cd2ap genotype \\
\hline CD2APwt & GCAGAGCTGTACTTTGGCCTTGTG & screening of chicken \\
\hline screen & & cd2ap genotype \\
\hline HIS & GAGCAAGCATGAGCACTGAAAACA & $\begin{array}{l}\text { screening of chicken } \\
\text { cd2ap genotype }\end{array}$ \\
\hline Blast-300 & GTGCAGTTTCGAATGGACAAAAGG & $\begin{array}{r}\text { screening of chicken } \\
\text { cd2ap genotype }\end{array}$ \\
\hline
\end{tabular}

\subsubsection{Vectors and constructs}

Common vector backbones and the constructs generated with it are listed in table 2.2 and 2.3 , respectively.

Table 2.2: Vectors

\begin{tabular}{lcr}
\hline vector & application & source/supplier \\
\hline pCRII Topo & T/A cloning & Invitrogen \\
pBluescript & $\begin{array}{c}\text { cloning of targeting } \\
\text { constructs }\end{array}$ & Stratagene \\
pBluescript-hisD & histidinol resistance cassette \\
for targeting constructs & J.-M. Buerstedde \\
pBluescript-bs & blasticidin resistance \\
& cassette for targeting \\
constructs & J.-M. Buerstedde
\end{tabular}




\begin{tabular}{|c|c|c|}
\hline pGEX4T1 & $\begin{array}{l}\text { expression of GST fusion } \\
\text { proteins }\end{array}$ & Ge Healthcare \\
\hline pMSCV puro & $\begin{array}{l}\text { expression of cDNA after } \\
\text { retroviral transduction }\end{array}$ & BD Biosciences Clontech \\
\hline pMSCV bleo & $\begin{array}{l}\text { expression of shRNAs after } \\
\text { retroviral transduction }\end{array}$ & $\begin{array}{r}\text { BD Biosciences Clontech, } \\
\text { Dr. M.Engelke }\end{array}$ \\
\hline pHCMV-VSV-G & expression of $v s v-g$ cDNA & M. Jücker \\
\hline LMP-pMSCV-GFP & $\begin{array}{c}\text { cloning and expression of } \\
\text { shRNA }\end{array}$ & OpenBiosystems \\
\hline
\end{tabular}

Table 2.3: Constructs

\begin{tabular}{|c|c|c|}
\hline construct & description & source/supplier \\
\hline pMSCVpuro & human SLP65, C-terminal & T.Oellerich \\
\hline hSLP65wt-GFP & GFP-tag & \\
\hline pMSCVpuro & human SLP65, R49A, & T.Oellerich \\
\hline hSLP65M1-GFP & C-terminal GFP-tag & \\
\hline pMSCVpuro & human SLP65, R248A, C-terminal & T.Oellerich \\
\hline hSLP65M2-GFP & GFP-tag & \\
\hline pMSCVpuro & human SLP65, R313A, C-terminal & T.Oellerich \\
\hline hSLP65M3-GFP & GFP-tag & \\
\hline pMSCVpuro & human SLP65, R248, 313A, & T.Oellerich \\
\hline hSLP65M23-GFP & C-terminal GFP-tag & \\
\hline pMSCVpuro & human SLP65, R49, 248, 313A, & T.Oellerich \\
\hline hSLP65M1-3-GFP & C-terminal GFP-tag & \\
\hline pGEX-4T-1 hCIN85 (SH3) 3 & $\begin{array}{l}\text { human CIN85, aa } 1-328, \quad \mathrm{~N}- \\
\text { terminal GST-tag }\end{array}$ & this thesis \\
\hline pGEX-4T-1 hCD2AP $(\mathrm{SH} 3)_{3}$ & $\begin{array}{l}\text { human CD2AP, aa } 1-330, \quad \mathrm{~N}- \\
\text { terminal GST-tag }\end{array}$ & this thesis \\
\hline pET15b hSLP65 & $\begin{array}{l}\text { human SLP65, N-terminal } \mathrm{His}_{6^{-}} \\
\text {tag }\end{array}$ & Dr. N. Herrmann \\
\hline pMSCVpuro & human SLP65, N-terminal Citrine- & this thesis \\
\hline
\end{tabular}




\begin{tabular}{|c|c|c|}
\hline Citrine-hSLP65wt & $\operatorname{tag}$ & Dr.M.Engelke \\
\hline pMSCVpuro & human SLP65, R248, 313A, N- & this thesis \\
\hline Citrine-hSLP65M23 & terminal Citrine-tag & Dr. M. Engelke \\
\hline pMSCVbleo & shRNA targeting bp 2389-2411 of & this thesis \\
\hline sh chCIN85_2389 & chicken cin85 mRNA & \\
\hline pMSCVbleo & unspecific shRNA & this thesis \\
\hline \multicolumn{3}{|l|}{ sh mock } \\
\hline LMP-pMSCV-GFP & shRNA targeting bp 2389-2411 of & this thesis \\
\hline sh chCIN85_2389 & chicken cin85 mRNA, IRES-GFP & \\
\hline LMP-pMSCV-GFP & unspecific shRNA, IRES-GFP & this thesis \\
\hline \multicolumn{3}{|l|}{ sh mock } \\
\hline pCRII Topo hCIN85 & human CIN85 & this thesis \\
\hline pCRII Topo hCIN85 $\Delta$ CC & human CIN85 $\Delta \mathrm{CC}$, aa 1-599 & this thesis \\
\hline pCRII Topo hCD2AP $\Delta C C$ & human $\mathrm{CD} 2 \mathrm{AP} \triangle \mathrm{CC}$, aa 1-547 & this thesis \\
\hline pMSCVpuro & human CD2AP $\triangle C C$, aa $331-639$, & this thesis \\
\hline Citrine-hCD2AP $\triangle \mathrm{CC}$ & N-terminal Citrine-tag & \\
\hline pMSCVpuro Citrine (C1) & $\mathrm{N}$-terminal Citrine & this thesis \\
\hline pCRII Topo hCD2AP & human CD2AP & this thesis \\
\hline pCRII Topo hCD2AP $\Delta$ SH3 & human CD2AP, aa $331-639$ & this thesis \\
\hline pCRII Topo hCIN85 $\Delta$ SH3 & human CIN85, aa 329-665 & this thesis \\
\hline pMSCVpuro & human $\quad \mathrm{CD} 2 \mathrm{AP}, \quad \mathrm{N}$-terminal & this thesis \\
\hline Citrine-hCD2AP & Citrine-tag & \\
\hline pMSCVpuro & human CD2AP $\triangle \mathrm{SH} 3$, aa 331 - & this thesis \\
\hline Citrine-hCD2AP $\triangle \mathrm{SH} 3$ & 639, N-terminal Citrine-tag & \\
\hline pMSCVpuro & human $\mathrm{CIN} 85 \Delta \mathrm{SH} 3$, aa 329-665, & this thesis \\
\hline Citrine-hCIN85 $\Delta \mathrm{SH} 3$ & N-terminal Citrine-tag & \\
\hline chicken CD2AP 15f6, 3431 & chicken cd2ap cDNA & Dr. J.M. Buerstedde \\
\hline pCRII Topo chCD2AP & chicken CD2AP & this thesis \\
\hline
\end{tabular}




\begin{tabular}{|c|c|c|}
\hline $\begin{array}{l}\text { pMSCVpuro } \\
\text { Citrine-chCD2AP }\end{array}$ & chicken CD2AP & this thesis \\
\hline $\begin{array}{l}\text { pABESpuro } \\
\text { mLyn_wt-HA }\end{array}$ & mouse Lyn, C-terminal HA-tag & Dr. M. Lösing \\
\hline $\begin{array}{l}\text { pABESpuro } \\
\text { mLyn_R275K-HA }\end{array}$ & $\begin{array}{l}\text { mouse Lyn, R275K substitution, } \\
\text { C-terminal HA-tag, kinase-dead }\end{array}$ & Dr. M. Lösing \\
\hline $\begin{array}{l}\text { pBluescript } \\
\text { cd2ap LARA HisD }\end{array}$ & $\begin{array}{l}\text { Histidinol D resistance cassette } \\
\text { with genomic sequences }\end{array}$ & Dr. K. Neumann \\
\hline $\begin{array}{l}\text { pBluescript } \\
\text { cd2ap LARA bs }{ }^{r}\end{array}$ & $\begin{array}{l}\text { Blasticidin } \mathrm{S} \text { resistance cassette } \\
\text { with genomic sequences }\end{array}$ & Dr.K. Neumann \\
\hline $\begin{array}{l}\text { pMSCVpuro } \\
\text { Citrine-tSH2 } 2 \text { N hSLP65_wt }\end{array}$ & $\begin{array}{l}\text { human Syk aa 1-277 (tSH2) fused } \\
\text { to hSLP65 aa } 49-456, \mathrm{~N} \text {-terminal } \\
\text { Citrine-tag }\end{array}$ & this thesis \\
\hline $\begin{array}{l}\text { pMSCVpuro } \\
\text { Citrine-tSH2 } \triangle \text { NhSLP65_M23 }\end{array}$ & $\begin{array}{l}\text { human Syk aa } 1-277 \text { (tSH2) fused } \\
\text { to hSLP65 aa } 49-456 \text {, R248, } \\
313 \mathrm{~A} \text { substitution, N-terminal } \\
\text { Citrine-tag }\end{array}$ & this thesis \\
\hline pCRII Topo SyktSH2 & $\begin{array}{l}\text { human Syk aa } 1-277 \text {, comprising } \\
\text { the tandem SH2 domains }\end{array}$ & Dr. I. Goldbeck \\
\hline $\begin{array}{l}\text { pGEX-4T-1 } \\
\text { chCD2AP ablong }\end{array}$ & $\begin{array}{l}\text { chicken CD2AP, aa } 350-640, N- \\
\text { terminal GST-tag, immunisation of } \\
\text { rabbits }\end{array}$ & this thesis \\
\hline $\begin{array}{l}\text { pMSCVpuro } \\
\text { Citrine } \Delta \mathrm{N} \text { hSLP65_wt }\end{array}$ & $\begin{array}{l}\text { human SLP65, aa 49-456, N- } \\
\text { terminal Citrine-tag }\end{array}$ & this thesis \\
\hline $\begin{array}{l}\text { pMSCVpuro } \\
\text { Citrine } \Delta \mathrm{N} \text { hSLP65_M23 }\end{array}$ & $\begin{array}{l}\text { human SLP65, aa 49-456, } \\
\text { R248,R313A, N-terminal Citrine- } \\
\text { tag }\end{array}$ & this thesis \\
\hline $\begin{array}{l}\text { pMSCVpuro Citrine } \\
\text { TIRAPwt- } \Delta \text { N hSLP65_wt }\end{array}$ & $\begin{array}{l}\text { human TIRAP, aa } 1-40 \mathrm{wt} \text {, fused } \\
\text { to hSLP65 aa } 49-456, \mathrm{~N} \text {-terminal } \\
\text { Citrine-tag }\end{array}$ & this thesis \\
\hline $\begin{array}{l}\text { pMSCVpuro Citrine } \\
\text { TIRAPwt- } \Delta \text { N hSLP65_M23 }\end{array}$ & $\begin{array}{l}\text { human TIRAP, aa } 1-40 \mathrm{wt} \text {, fused } \\
\text { to hSLP65 aa } 49-456 \text {, }\end{array}$ & this thesis \\
\hline
\end{tabular}




\begin{tabular}{|c|c|c|}
\hline & $\begin{array}{l}\text { R248,R313A, N-terminal Citrine- } \\
\text { tag }\end{array}$ & \\
\hline $\begin{array}{l}\text { pMSCVpuro Citrine } \\
\text { mutTIRAP- } \triangle N \text { hSLP65_wt }\end{array}$ & $\begin{array}{l}\text { human TIRAP, aa } 1-40 \text { Kall, fused } \\
\text { to hSLP65 aa } 49-456, \text { N-terminal } \\
\text { Citrine-tag }\end{array}$ & this thesis \\
\hline $\begin{array}{l}\text { pMSCVpuro Citrine } \\
\text { mutTIRAP- } \Delta \text { N hSLP65_M23 }\end{array}$ & $\begin{array}{l}\text { human TIRAP, aa } 1-40 \text { wtKall, } \\
\text { fused to hSLP65 aa } 49-456 \text {, } \\
\text { R248A, R313A,N-terminal Citrine- } \\
\text { tag }\end{array}$ & this thesis \\
\hline hTIRAP-SLP65 & human TIRAP & Dr. N. Herrmann \\
\hline hTIRAP Kall-SLP65 & human TIRAP, K15,16,31,32A & Dr. N. Herrmann \\
\hline
\end{tabular}

\subsubsection{Additional material}

8-well chamber slides

GeneRuler 1kb DNA ladder

Glutathione sepharose ${ }^{\circledR}$ 4Fast Flow

Nitrocellulose filter Hybond ECL ${ }^{\mathrm{TM}}$

Prestained protein marker, Broad Range

Sterile filter

\subsubsection{Instruments}

Agarose Gelelectrophoresis System

Autoclaves

BioPhotometer

Chemilux Gel Imager

Electrophoresis power supply

Electroporation cuvette (4mm gap)
LabTek $^{\text {TM }}$, Nunc

Fermentas

GE Healthcare

Amersham Biosciences

New England Biolabs ${ }^{\circledR}$

Roth

Peqlab Biotechnology

IBS Integra Biosciences

Eppendorf

Intas Science Imaging

Amersham BioSciences

Peqlab Biotechnology 
FACSCalibur

GenePulser ${ }^{\circledR}$ II electroporation system

HeraCell ${ }^{\circledR} 150 \mathrm{CO}_{2}$ incubator

HERAsafe ${ }^{\circledR}$ Microbiological safety cabinet

LSR II

Mastercycler epgradient \& personal

Microbiological Incubator $\left(37^{\circ} \mathrm{C}\right)$

Microcentrifuge 5415D

Microscope

Mini-gel apparatus

Multifuge 3SR

Neubauer counting chamber

ph-Meter $\mathrm{pH}$ Level 1

Refrigerated centrifuge RC 3B Plus

Refrigerated Microcentrifuge 5417R

Semi-dry transfer unit TE 70

Sonificator
Becton Dickinson

Bio-Rad Laboratories

Heraeus

Heraeus

Becton Dickinson

Eppendorf

Heraeus, Thermo Scientific

Eppendorf

Leitz $^{\circledR}$

Bio-Rad Laboratories

Heraeus

Brand $\mathrm{GmbH}$

inoLab $^{\circledR}$

Sorvall ${ }^{\circledR}$

Eppendorf

Hoefer

Bandelin

Spectral confocal \& multiphoton system TCS SP2 Leica Microsystems

ThermoStat plus

Unitron incubation shaker

UVette

Vortex-Genie 2

Water bath

Water Purification System Milli-Q

Westernblotting filter paper
Eppendorf

Infors

Eppendorf

Scientific Industries, Inc.

Schütt Labortechnik

Millipore $^{\mathrm{TM}}$, Sartorius

Schleicher \& Schell 


\subsubsection{Software}

Adobe ${ }^{\circledR}$ Photoshop ${ }^{\circledR}$ CS5

Image editing

CellQuestPro

FlowCytometry Analysis

CorelDRAW Graphics Suite X5

Graphic editing

Endnote 7

Bibliography

FACS Diva

FlowCytometry Analysis

FlowJo

FlowCytometry Analysis

ImageJ

Image Processing and Analysis

Leica Confocal Software

Confocal imaging software

MS Office

pDRAW 32

DNA analysis

\subsubsection{Websites and databases}

http://expasy.org/

http://genome.ucsc.edu/cgi-bin/hgBlat

http://katahdin.cshl.org:9331/homepage/siRNA/RNAi.cgi?type=shRNA

http://multalin.toulouse.inra.fr/multalin/

http://scansite.mit.edu/motifscan_seq.phtml

http://www.ncbi.nlm.nih.gov/

http://www.phosphosite.org 


\subsection{Methods}

\subsubsection{Methods in Molecular Biology}

\subsubsection{Fragmentation of DNA with restriction endonucleases (typell)}

For site-specific cleavage and generation of $3{ }^{`}$-hydroxyl and $5{ }^{`}$-phosphate termini substrate DNA was incubated with restriction endonucleases according to the manufacturer's instructions (New England Biolabs ${ }^{\circledR}$ ).

\subsubsection{Purification of DNA fragments}

DNA fragments of $50 \mathrm{bp}$ to $15 \mathrm{~kb}$ were purified from agarose gels or directly from PCR reactions with Wizard $^{\circledR}$ SV Gel and PCR Clean-Up System (Promega) according to

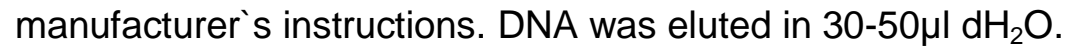

\subsubsection{Ligation of DNA fragments}

For the formation of phosphodiester bonds between linearised vector and insert DNA molecules T4 DNA ligase (New England Biolabs ${ }^{\circledR}$ ) was used. A $10 \mu$ l reaction was set up mixing vector and insert molecules in a 1:3 molar ratio and T4 DNA ligase in ATP-containing T4 DNA ligase buffer for $>1 \mathrm{~h}$ at $\mathrm{RT}$ or $16^{\circ} \mathrm{C}$ overnight. $5 \mu$ of the mixture were used for transformation of competent cells.

\subsubsection{Phenol-Chloroform DNA extraction}

Protein contaminations were removed from nucleic acid samples by adding an equal volume of Phenol-Chloroform-Isoamyl alcohol (2:3:4) followed by a short centrifugation step for phase separation. The aqueous, DNA-containing phase was transferred into a new reaction tube and DNA was precipitated with ethanol.

\subsubsection{Ethanol precipitation of DNA}

To precipitate DNA from aqueous solutions samples were mixed with $1 / 10$ volume $5 \mathrm{M} \mathrm{NaCl}$ and 2.5 volumes $100 \%$ ethanol $\left(-20^{\circ} \mathrm{C}\right)$, vortexed and incubated at $-80^{\circ} \mathrm{C}$ for $20 \mathrm{~min}$. Samples were centrifuged at $20.000 \mathrm{xg}$ at $4^{\circ} \mathrm{C}$ for $10 \mathrm{~min}$. The supernatant was discarded and the DNA pellet was air-dried before being dissolved in PBS. 


\subsubsection{Agarose gel electrophoresis of nucleic acids}

Agarose gel electrophoresis was used to separate DNA fragments according to their size. $0.7 \%-2 \%(\mathrm{w} / \mathrm{v})$ agarose was molten in TAE buffer and ethidium bromide was added to a final concentration of $0.5 \mu \mathrm{g} / \mathrm{mL}$. DNA samples were mixed with $6 \mathrm{x}$ DNA loading buffer, loaded on the gel and electrophoresis was performed at a voltage of $5 \mathrm{~V} / \mathrm{cm}$ distance between electrodes. A DNA molecular weight standard (GeneRuler $1 \mathrm{~kb}$ DNA ladder, MBI Fermentas) was loaded for determination of DNA size and quantity.

TAE buffer

6x DNA loading buffer
40mM Tris/acetic acid, pH 7.8; 10mM NaAc; $1 \mathrm{mM}$ EDTA, $\mathrm{pH} 8$

$10 \mathrm{mM}$ Tris- $\mathrm{HCl}(\mathrm{pH} 7.6), 0.03 \%$ bromophenol blue, $60 \%$ glycerol

\subsubsection{Photometric determination of DNA concentration}

Concentration of double-stranded DNA was determined with an Eppendorf photometer in quartz cuvettes and calculated using the following equation:

$1 \mathrm{~A}_{260}$ Unit of dsDNA $=50 \mu \mathrm{g} \mathrm{DNA} / \mathrm{mL}$ diluent

The purity of DNA can be estimated by the following value:

Pure DNA : $A_{260} / A_{280} \geq 1.8$

\subsubsection{Dephosphorylation of vector DNA}

To prevent religation of vector DNA which was cut with only one restriction endonuclease $1 \mu \mathrm{l}$ calf intestine phosphatase (CIP) was added to the restriction mixture and incubated for $1 \mathrm{~h}$ at $37^{\circ} \mathrm{C}$. Dephosphorylated vector was purified after gel electrophoresis from agarose.

\subsubsection{Extraction and purification of plasmid DNA from E.coli}

The small scale purification of plasmid DNA (Miniprep) was based on alkaline lysis using the Invisorb ${ }^{\circledR}$ Spin Plasmid Mini Two Kit (Invitek) according to manufacturer's instructions. Therefore $4 \mathrm{~mL}$ of LB-medium supplemented with the appropriate antibiotic were inoculated with a single bacterial colony and grown in a shaker at $37^{\circ}$ and $180 \mathrm{rpm}$ overnight. For preparative isolation of plasmid DNA (Midiprep) $100 \mathrm{~mL}$ bacterial culture were harvested and DNA purification was performed with Wizard ${ }^{\circledR}$ Plus SV Midiprep Kit (Promega) according to manufacturer`s instructions. DNA was eluted with nuclease-free $\mathrm{H}_{2} \mathrm{O}$. 
LB medium/Amp: $\quad 10 \mathrm{~g}$ tryptone, $5 \mathrm{~g}$ Yeast extract, $10 \mathrm{~g} \mathrm{NaCl}$ ad $1000 \mathrm{~mL} \mathrm{H}_{2} \mathrm{O} ; 100 \mu \mathrm{g} / \mathrm{mL}$ Ampicillin

\subsubsection{Cloning of PCR fragments via T/A cloning}

PCR products generated by taq polymerase comprise additional deoxyadenosines at the 3 'end. This allows ligation into linearised $\mathrm{pCR}^{\circledR}$ II-TOPO ${ }^{\circledR}$ having single, overhanging 3 'deoxythymidine residues. PCR products generated by proof-reading Phusion polymerase were purified from agarose gels, and mixed 1:1 with Taq PCR Master Mix Kit to produce $33^{`}-\mathrm{A}$ overhangs. PCR products were ligated into linearised $\mathrm{pCR}^{\circledR}$ II-TOPO ${ }^{\circledR}$ according to manufacturer's instructions.

\subsubsection{Isolation of genomic DNA from DT40 B cells}

For analysis of DT40 genotypes by PCR $0.5-1 \cdot 10^{6}$ cells were harvested, washed with PBS and resuspended in 20-50 $\mu$ l Proteinase-K buffer. The suspension was incubated at $56^{\circ} \mathrm{C}$ for $1 \mathrm{~h}$ and proteinase $\mathrm{K}$ was inactivated at $95^{\circ} \mathrm{C}$ for $10 \mathrm{~min}$. Genomic DNA was used in PCR and stored at $4^{\circ} \mathrm{C}$.

Proteinase $\mathrm{K}$ buffer $\quad 1 \%(\mathrm{v} / \mathrm{v})$ proteinase $\mathrm{K} ; 1 \mathrm{x}$ polymerase buffer; $0.5 \%$ Tween

\subsubsection{Preparation of chemo-competent E.coli}

To obtain stocks of competent E.coli strains $5 \mathrm{~mL}$ LB medium were inoculated with the E.coli strain and grown in a shaker at $37^{\circ}$ and $180 \mathrm{rpm}$ overnight. The next day $900 \mu \mathrm{l}$ overnight culture were transferred into $150 \mathrm{~mL}$ LB medium and grown until an $\mathrm{OD}_{600}=0.45-0.55$ was reached. The culture was put on ice for $10 \mathrm{~min}$ and bacteria harvested at $5,000 \mathrm{xg}$ at $4^{\circ} \mathrm{C}$ for $10 \mathrm{~min}$. The medium was decanted and bacteria were resuspended in $30 \mathrm{~mL}$ TFB I. After $10 \mathrm{~min}$ incubation on ice cells were recovered by centrifugation and resuspended in $6 \mathrm{~mL}$ icecold TFB II. $50 \mu \mathrm{l}$ aliquots of this suspension were flash frozen with liquid nitrogen and stored at $-80^{\circ} \mathrm{C}$.

LB medium: $10 \mathrm{~g}$ tryptone, $5 \mathrm{~g}$ Yeast extract, $10 \mathrm{~g} \mathrm{NaCl}$ ad $1000 \mathrm{~mL} \mathrm{H}_{2} \mathrm{O}$

TFB I: $\quad 50 \mathrm{mM} \mathrm{MnCl} \cdot 4 \mathrm{H}_{2} \mathrm{O} ; 100 \mathrm{mM} \mathrm{KCl} ; 10 \mathrm{mM} \mathrm{CaCl} 2 \cdot 2 \mathrm{H}_{2} \mathrm{O} ; 30 \mathrm{mM} \mathrm{KOAc}, \mathrm{pH} 6.0$; $15 \%$ (v/v) glycerol; adjust to $\mathrm{pH} 6.1$ with $\mathrm{HOAc}$; autoclaved; $4^{\circ} \mathrm{C}$ storage

TFB II: $\quad 75 \mathrm{mM} \mathrm{CaCl} 2 \cdot 2 \mathrm{H}_{2} \mathrm{O} ; 10 \mathrm{mM} \mathrm{KCl} ; 10 \mathrm{mM}$ MOPS; $15 \%(\mathrm{v} / \mathrm{v})$ glycerol; adjust to $\mathrm{pH} 7.0$ with $\mathrm{KOH}$; autoclaved; $4^{\circ} \mathrm{C}$ storage 


\subsubsection{Transformation of chemo-competent E.coli}

To cause circular DNA to enter E.coli, competent bacteria were thawed on ice and 150ng of plasmid DNA or $5 \mu$ l of a ligation reaction were added and mixed gently by flicking the tubes. The mixture was stored on ice for $20 \mathrm{~min}$, heat-shocked for $70 \mathrm{~s}$ at $42^{\circ} \mathrm{C}$ and returned to ice for $2 \mathrm{~min}$. Cells were resuspended in $150 \mu \mathrm{LB}$ medium and the culture was grown at $37^{\circ} \mathrm{C}$ for $20 \mathrm{~min}$. Subsequently bacteria were spread onto LB plates containing an appropriate antibiotic and incubated at $37^{\circ} \mathrm{C}$ overnight. After transformation of $\mathrm{pCR}^{\circledR}$ II-TOPO ${ }^{\circledR}$ vectors LB plates were treated with IPTG/X-Gal before spreading of the bacteria to allow blue-white screening of the clones.

LB plates:

$20 \mathrm{~g}$ agar/1000mL LB; autoclaved and cooled to $60^{\circ} \mathrm{C}$; addition of $50-100 \mu \mathrm{g} / \mathrm{mL}$ ampicillin; $4^{\circ} \mathrm{C}$ storage

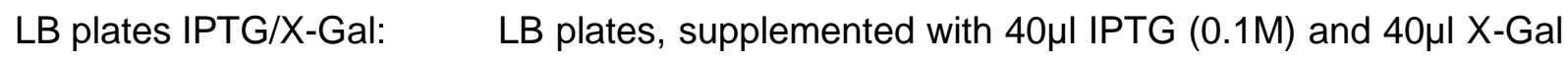
$(40 \mathrm{mg} / \mathrm{mL})$

\subsubsection{Long term storage of bacteria}

Fresh bacterial cultures were mixed with $50 \%(\mathrm{v} / \mathrm{v})$ glycerol (autoclaved) and stored at $-80^{\circ} \mathrm{C}$.

\subsubsection{In vitro amplification of DNA by the Polymerase Chain Reaction (PCR)}

The PCR is a technique used for DNA analysis and molecular cloning and is based on the in vitro amplification of a specific segment of DNA (Mullis et al. 1986). The basic reaction includes three repetitive temperature steps allowing denaturation of dsDNA, primer annealing and elongation by a polymerase.

For molecular cloning and site-directed mutagenesis PCR reactions were performed using thermo stable Phusion ${ }^{\circledR}$ High-Fidelity DNA Polymerase (Finnzymes, Thermo Fisher Scientific) according to manufacturer`s instructions.

\subsection{Analysis of $c d 2 a p^{-/-}$DT40 B cell line}

Genetic analysis of $c d 2 a p^{-/}$DT40 B cell clones was performed with Taq PCR Master Mix Kit. Freshly isolated genomic DNA was screened for targeted integration of resistance cassettes using the following PCR setups and program: 
Table 2.4: PCR setup

\begin{tabular}{|c|c|c|}
\hline wt allele & his $D^{r}$ integration & bs $^{r}$ integration \\
\hline 1x Taq PCR Master Mix & 1x Taq PCR Master Mix & 1x Taq PCR Master Mix \\
\hline $0.5 \mu \mathrm{l}$ genomic DNA & $0.5 \mu \mathrm{l}$ genomic DNA & $0.5 \mu l$ genomic DNA \\
\hline $0.1 \mu \mathrm{l}$ Primer $(100 \mu \mathrm{M})$ & $0.1 \mu$ l Primer $(100 \mu \mathrm{M})$ & $0.1 \mu \mathrm{l}$ Primer $(100 \mu \mathrm{M})$ \\
\hline CD2APRAscreen & CD2APLAscreen & CD2APLAscreen \\
\hline $0.1 \mu$ Primer & $0.1 \mu \mathrm{l}$ Primer & $0.1 \mu$ l Primer \\
\hline CD2APwt & HIS & Blast-300 \\
\hline ad $20 \mu \mathrm{l} \mathrm{H}_{2} \mathrm{O}$ & ad $20 \mu \mathrm{l} \mathrm{H}_{2} \mathrm{O}$ & ad $20 \mu \mathrm{l} \mathrm{H}_{2} \mathrm{O}$ \\
\hline
\end{tabular}

Table 2.5: PCR program

\begin{tabular}{ccc}
\hline step & temperature & time \\
\hline initial denaturation & $95^{\circ} \mathrm{C}$ & $2 \mathrm{~min}$ \\
denaturation & $95^{\circ} \mathrm{C}$ & $15 \mathrm{sec}$ \\
primer annealing & $63^{\circ} \mathrm{C}$ & $30 \mathrm{sec}$ \\
elongation & $72^{\circ} \mathrm{C}$ & $4 \mathrm{~min}$ \\
final elongation & $72^{\circ} \mathrm{C}$ & $10 \mathrm{~min}$ \\
number of cycles & & \\
\hline
\end{tabular}

\subsection{Site directed mutagenesis}

To introduce point mutations in CDNA, the respective vectors were subjected to PCR using two oligonucleotide primers containing the desired mutation in the middle. Elongation of the complementary oligonucleotides with Phusion $^{\circledR}$ High-Fidelity DNA Polymerase results in generation of a mutated vector. The PCR reaction was incubated with $1 \mu \mathrm{L} D \mathrm{D} /$ endonuclease for $1 \mathrm{~h}$ at $37^{\circ} \mathrm{C}$ to digest the parental, methylated DNA template plasmid. The reaction was transformed into chemo-competent E.coli and plasmids from individual clones were analysed for the introduction of the desired mutation by sequencing.

\subsubsection{DNA sequence analysis}

Sequencing was performed by Seqlab-Sequence laboratories Göttingen GmbH (Göttingen, Germany). 
2.2.1.17 RNA interference (RNAi) with cin85 expression using microRNA-30 adapted shRNAmir retroviral vectors

RNA interference is used by cells in order to control gene expression by either interfering with messenger RNA stability or its translation (Cullen 2005). This mechanism can be hijacked by introduction of vectors encoding shRNAs resulting in down regulation of the expression of a gene of interest. To suppress gene expression of chicken cin85 a microRNA30-adapted (miR-30) retroviral transduction system was chosen that is based on RNAi via short hairpin (sh) RNA (Open Biosystems (Dickins et al. 2005)). An shRNA targeting sequence directed against the 3 UTR of chicken cin85 mRNA was incorporated into a 97mer DNA oligonucleotide. The oligonucleotide finally comprised the sense targeting sequence (black), a miR-30 loop structure (green) and the antisense targeting sequence (blue), flanked $5^{`}$ and $3^{`}$ by a miR-30 context (red) (TGCTGTTGACAGTGAGCGCTGACTG AGACTCAAATTTATGTAGTGAAGCCACAGATGTACATAAATTTGAGTCTCAGTCATTGCC TACTGCCTCGGA).

According to manufacturer's instructions the oligonucleotide was used as template in a PCR for introduction of restriction sites and was subsequently cloned into the LMP miR-30 adapted vector encoding a GFP marker for viral integration (LMP-pMSCV-GFP, Catalogue Number: EAV4071). As a control an unspecific mock sequence was carried along (mock, TGCTGTTGACAGTGAGCGATCTCGCTTGGGCGAGAGTAAGTAGTGAAGCCACAGATGT ACTTACTCTCGCCCAAGCGAGAGTGCCTACTGCCTCGGA) (Paddison et al. 2004).

For the reduction of cin85 expression followed by genetic reconstitution (see figure 3.6), vectors were used, that encode the miR-30-adapted shRNA and bleomycin resistance cassette, but no GFP marker. Therefore the anti-cin85 shRNA coding sequence was cut off the LMP-pMSCV-GFP vector via Bglll and Agel and cloned into likewise linearised pMSCVbleo vector. All generated vectors were used for retroviral gene transfer (see 2.2.2.8) and expression resulted in the formation of a short hairpin that is processed by the cellular RNAi pathway.

\subsubsection{Cell culture methods}

\subsubsection{Cell culture material}

Tissue culture equipment

Greiner Bio-One, Nunc, Sarstedt

Bleocin $^{\mathrm{TM}}$ antibiotic Calbiochem $^{\circledR}$

Puromycin antibiotic InvivoGen

Blasticidin S antibiotic InvivoGen 
Histidinol D antibiotic

RPMI

DMEM

FCS

CS

Penicillin

Streptomycin

Trypsin/EDTA
Sigma-Aldrich $^{\circledR}$

Gibco $^{\circledR}$

Gibco $^{\circledR}$

Gibco $^{\circledR}$

Gibco $^{\circledR}$

Gibco $^{\circledR}$

Gibco $^{\circledR}$

Invitrogen ${ }^{\mathrm{TM}}$

\subsubsection{Cell culture conditions}

All cell lines were cultured in a $5 \% \mathrm{CO}_{2}$ humidified atmosphere at $37^{\circ} \mathrm{C}$. FCS and CS were heated to $56^{\circ} \mathrm{C}$ for $30 \mathrm{~min}$ for inactivation of complement factors. DT40 B cells were cultured in RPMI1640+Glutamax, supplied with 10\%FCS, $1 \%$ CS, 50U/mL Penicillin and $50 \mu \mathrm{g} / \mathrm{mL}$ Streptomycin. Platinum-E cells were nutritioned with DMEM+Glutamax containing $10 \%$ FCS, $10 \mu \mathrm{g} / \mathrm{mL}$ blasticidin, $2.5 \mu \mathrm{g} / \mathrm{mL}$ puromycin, $50 \mathrm{U} / \mathrm{mL}$ Penicillin and $50 \mu \mathrm{g} / \mathrm{mL}$ Streptomycin. Confluent cells were washed with PBS and detached with Trypsin/EDTA solution for passaging. Centrifugation of eukaryotic cells was performed at $1200 \mathrm{rpm}$ for $4 \mathrm{~min}$ at $4^{\circ} \mathrm{C}$. All cell culturing was conducted under sterile conditions in laminar flow hoods and with sterile equipment.

\subsubsection{Cell lines}

\subsection{DT40 B cell line $\left(\right.$ ATCC $^{\circledR}$ Number: CRL-2111 ${ }^{\mathrm{TM}}$ )}

The DT40 B cell line is an avian leukosis virus induced bursal lymphoma cell line (Baba and Humphries 1984). The DT40 B cell line shows an increased ratio of targeted to random integration of targeting constructs in the homologous gene loci allowing relatively straightforward genetic modification of a vertebrate cell line. The ease of homologous recombination has resulted in a long list of knock out cell lines making this cell line a powerful tool for molecular B cell immunology (Winding and Berchtold 2001). 
Table 2.6: DT40 knock-out cell lines

\begin{tabular}{|c|c|}
\hline DT40 cell line & reference \\
\hline cd2ap ${ }^{-/-}$ & this thesis \\
\hline$g r b 2^{-1}$ & (Hashimoto et al. 1998) \\
\hline lyn ${ }^{-/}$ & (Takata et al. 1994) \\
\hline $\operatorname{slp} 65^{\%}$ & (Ishiai et al. 1999a) \\
\hline syk ${ }^{-/}$ & (Takata et al. 1994) \\
\hline
\end{tabular}

\subsection{Platinum-E}

Platinum-E is a retrovirus packaging cell line derived from HEK293T cells. The cell line expresses the viral structural genes gag-pol and env of moloney murine leukaemia virus from two different vectors (either coding for blasticidin or puromycin resistance) decreasing the probability for the generation of replication-competent viruses (Morita et al. 2000).

\subsubsection{Freezing and thawing of eukaryotic cells}

For freezing $0.5-1 \cdot 10^{7}$ cells were harvested and resuspended in $1 \mathrm{~mL}$ freezing medium. The cell suspension was transferred into cryo conservation tubes and put on ice. For long-term storage cells were frozen at $-140^{\circ} \mathrm{C}$.

Cells were thawed rapidly at $37^{\circ} \mathrm{C}$ put in $10 \mathrm{~mL}$ culture medium to dilute the DMSO and centrifuged. The cellular pellet was resuspended in $10 \mathrm{~mL}$ fresh culture medium and transferred on a culture dish.

Freezing medium $\quad 90 \%$ FCS $(v / v), 10 \% \mathrm{DMSO}(\mathrm{v} / \mathrm{v})$

\subsubsection{Counting of suspension cells}

Cells were mixed thoroughly and $10 \mu \mathrm{l}$ of the suspension were counted in a neubauer chamber.

\subsubsection{Transfection of DT40 B cells by electroporation}

$1-2 \cdot 10^{7}$ cells were harvested and resuspended in $700 \mu$ PBS. After mixing with $25 \mu \mathrm{g}$ of linearised DNA the cell suspension was transferred to an electroporation cuvette and placed on ice for $10 \mathrm{~min}$. Electroporation was performed at $270 \mathrm{~V}$ and $960 \mu \mathrm{F}$. The cells were put on 
ice for $10 \mathrm{~min}$ and resuspended in cell culture medium. After an incubation at $37^{\circ} \mathrm{C}$ for $24 \mathrm{~h}$ the cell culture medium was changed and $1 \mu \mathrm{g} / \mathrm{mL}$ puromycin was added as antibiotic and the cells were plated on two 96 -well-dishes with $200 \mu \mathrm{l} /$ well. After 7-14 days stable transfectants were visible and grown for further analysis.

\subsubsection{Transfection of Platinum-E cells by lipofection}

Platinum-E cells stably express gag-pol and env viral structural genes. To be used as retroviral packaging cell line, cells were transfected with retroviral vectors (pMSCV, derived from Moloney murine leukaemia virus, (Hawley et al. 1994)) providing the packaging signal $\Psi^{+}$, transcription and processing elements and a gene of interest. In addition a vector coding for the envelope glycoprotein of the vesicular stomatitis virus (VSV-G) is co-transfected to allow production of amphotrophic viruses (Emi et al. 1991). Platinum-E cells were split to 50$70 \%$ confluency in a $6 \mathrm{~cm}$ dish. The next day the lipofection mixture composed of $180 \mu \mathrm{IPPMI}$ w/o supplements, $7.5 \mu \mathrm{l}$ TransI ${ }^{\circledR}-293$ Transfection Reagent (Mirus Bio LLC), 1.8 $\mu$ g retroviral expression vector and $0.7 \mu \mathrm{g}$ pHCMV-VSV-G was incubated for $30 \mathrm{~min}$ at RT. The Platinum-E culture medium was exchanged with $4 \mathrm{~mL}$ DT40 culture medium and the lipofection mixture was added drop wise to the cells. After $48 \mathrm{~h}$ incubation at $37^{\circ} \mathrm{C}$ and $5 \% \mathrm{CO}_{2}$ the supernatant was used for retroviral transduction.

\subsubsection{Retroviral transduction of DT40 B cells}

Retroviral gene transfer (Ausubel et al. 1995) was used to introduce stable genetic material into the genome of DT40 B cells. Therefore $1-2 \cdot 10^{6}$ DT40 B cells were resuspended in $2 \mathrm{~mL}$ fresh DT40 culture medium and the $4 \mathrm{~mL}$ retrovirus-containing supernatant (see 2.2.2.7) was added after sterile filtration $(0.45 \mu \mathrm{m}$ pore size). To increase infectivity polybrene was added to a concentration of $3 \mu \mathrm{g} / \mathrm{mL}$. Cells were incubated for $24 \mathrm{~h}$ under standard cell culture conditions. To remove remaining retrovirus the cells were centrifuged and resuspended in $10 \mathrm{~mL}$ fresh culture medium and $24 \mathrm{~h}$ later selected with the appropriate antibiotics (puromycin $1 \mu \mathrm{g} / \mathrm{mL}$ for 3 days, blasticidin $30 \mathrm{mg} / \mathrm{mL}$ for 3 days, bleocin $70 \mu \mathrm{g} / \mathrm{mL}$ for 48h)(Ausubel et al. 1995).

All manipulations of pseudo-typed retroviruses were performed in compliance with the S2 standard and safety instructions.

Polybrene stock solution

$3 \mathrm{mg} / \mathrm{mL}$ polybrene in PBS; sterile-filtered; freshly prepared 


\subsubsection{Flow cytometry}

\subsubsection{Fluorophor expression analysis}

To monitor the expression of fluorophor-tagged proteins $1 \cdot 10^{6}$ cells were washed, resuspended in PBS and analysed by flow cytometry.

\subsubsection{Surface chicken IgM internalisation assay}

To monitor the amount of surface $\operatorname{lgM} 4 \cdot 10^{6}$ DT40 B cells were washed with ice-cold PBS and resuspended in $100 \mu \mathrm{l}$ PBS containing $2 \mu \mathrm{g} / \mathrm{mL}$ anti-chicken IgM (M4, mouse IgM). After $15 \mathrm{~min}$ incubation on ice cells were washed three times with $1 \mathrm{~mL}$ ice-cold PBS and resuspended in $400 \mathrm{~mL}$ ice-cold DT40 medium. $100 \mu \mathrm{l}$ of the cell suspension were put in $400 \mu$ l pre-warmed DT40 medium and incubated at $37^{\circ} \mathrm{C}$ for 5,10 or $20 \mathrm{~min}$. For the $0 \mathrm{~min}$ time point $400 \mu \mathrm{l}$ ice-cold DT40 medium was added. Cells were washed with $1 \mathrm{~mL}$ ice-cold PBS and centrifuged at $300 \mathrm{xg}$ for $4 \mathrm{~min}$ at $4^{\circ} \mathrm{C}$. To stain residual surface IgM bound by M4 cell pellets from different time points were resuspended in 50 $\mu$ PBS containing goat antimouse IgM Cy ${ }^{\mathrm{TM}} 5$-conjugated $\mathrm{F}\left(\mathrm{ab}^{\prime}\right)_{2}$ fragment in a 1:200 dilution and incubated on ice for

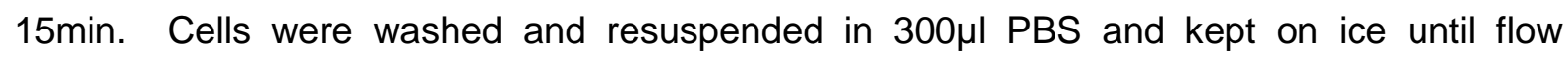
cytometric analysis.

\subsubsection{3 $\mathrm{Ca}^{2+}$ mobilisation analysis}

For monitoring changes in $\mathrm{Ca}^{2+}$ levels of $\mathrm{B}$ lymphocytes flow cytometry (LSR II, Becton Dickinson) was applied using the polycyclic chelator Indo-1-AM. As Indo-1 is an uncharged molecule it is able to permeate membranes, but it is trapped inside the cell upon non-specific cleavage by cytosolic esterases (dye-trapping). The fluorescence properties of this ratio metric dye is markedly changed when $\mathrm{Ca}^{2+}$ is bound; the emission maximum from Indo-1-AM shifts from $\sim 475 \mathrm{~nm}$ in $\mathrm{Ca}^{2+}$-free (Indo blue) medium to $400 \mathrm{~nm}$ (Indo violet) when the dye is saturated with Calcium-Ions. The use of the 400/475 emission ratio reduces the effects of unequal dye loading, leakage of dye and photo bleaching.

For $\mathrm{Ca}^{2+}$ mobilisation analysis $1.5 \cdot 10^{6}$ cells were harvested at $300 \mathrm{xg}$ for $4 \mathrm{~min}$ at $\mathrm{RT}$. The cell pellet was resuspended in $600 \mu$ DT40 cell culture medium loaded with $0.015 \%$ Pluronic F127 and $1 \mu \mathrm{M}$ Indo- 1 -AM and incubated for $20 \mathrm{~min}$ at $20^{\circ} \mathrm{C}$. Cells were incubated at $37^{\circ} \mathrm{C}$ for 10 min and washed $1 \mathrm{x}$ with $\mathrm{Ca}^{2+}$-containing Krebs-Ringer solution, resuspended in $600 \mu \mathrm{l}$ $\mathrm{Ca}^{2+}$-containing Krebs-Ringer solution and kept at $25^{\circ} \mathrm{C}$ for $15 \mathrm{~min}$ and analysed by flow cytometry. After $20 \mathrm{~s}$ of recording cells were stimulated with M4 in $\mathrm{Ca}^{2+}$-containing KrebsRinger solution (concentration indicated in the figure) for 3-5 minutes. The $\mathrm{Ca}^{2+}$ mobilisation 
profiles were analysed using FlowJo and Excel software. Kinetic graphs in each figure represent the median of all cells analysed in a given second.

For $\mathrm{Ca}^{2+}$ mobilisation analysis in the genetic reconstitution experiments as depicted in figure 3.6 Citrine expression was monitored in parallel and kinetic graphs were derived after gating for Citrine positive or negative populations resulting in two graphs from one measurement.

Krebs-Ringer solution $\left(\mathrm{Ca}^{2+}\right) \quad$ 10mM HEPES, $\mathrm{pH} 7.0 ; 140 \mathrm{mM} \mathrm{NaCl} ; 4 \mathrm{mM} \mathrm{KCl} ; 1 \mathrm{mM}$ $\mathrm{MgCl}_{2} ; 10 \mathrm{mM}$ glucose

Indo-1-AM stock

$1 \mathrm{mM}$ in DMSO; store at $-20^{\circ} \mathrm{C}$

Pluronic F-127 stock

$5 \%(w / v)$

\subsubsection{Confocal laser scanning microscopy}

Confocal laser scanning microscopy of Citrine fusion proteins $0.5-1 \cdot 10^{6}$ cells were washed and resuspended in $200 \mu \mathrm{l}$ with $\mathrm{Ca}^{2+}$-containing Krebs-Ringer solution. Cells were analysed in 8-well chamber slides (LabTek ${ }^{\mathrm{TM}}$, Nunc) using the Leica TCS SP2 microscope (Leica objective PL APO 63x1.3 Glycerol HCX) and Leica Confocal Software. The Citrine fluorophor was excited at $514 \mathrm{~nm}$ and emission was collected from $530-600 \mathrm{~nm}$. Pictures were taken prior to BCR stimulation or after carefully adding 40 $\mu$ M4-containing Krebs-Ringer solution to a final concentration of $2 \mu \mathrm{g} / \mathrm{mL}$. Images were exported to Adobe ${ }^{\circledR}$ Photoshop $^{\circledR}$ CS5.

Quantification of fluorescence intensities in DT 40 B cells was performed using the ImageJ software. The mean fluorescence intensity (MFI) at the plasma membrane was normalised to the MFI in the cytosol for each cell. The mean of the normalised MFI at the plasma membrane in resting or BCR-stimulated cells is indicated as $M_{\text {rest. }}$ or $M_{\text {stim. }}$, respectively. The fold-change expresses the change of the MFI at the plasma membrane before and after BCR-stimulation calculated for each cell.

$$
\begin{aligned}
& M_{\text {rest. }}=\frac{1}{n} \cdot \sum_{i=1}^{n} x_{i}, \quad x=\left(\frac{M F I_{\text {plasma membrane }}}{M F I_{\text {cytosol }}}\right)_{\text {resting cell }} \\
& M_{\text {stim. }}=\frac{1}{n} \cdot \sum_{i=1}^{n} x_{i}, \quad x=\left(\frac{M F I_{\text {plasma membrane }}}{M F I_{\text {cytosol }}}\right)_{\text {stimulated cell }}
\end{aligned}
$$


fold change $=\frac{1}{n} \cdot \sum_{i=1}^{n} x_{i}, \quad x=\frac{\left(\frac{M F I_{\text {plasma membrane }}}{M F I_{\text {cytosol }}}\right)_{\text {stimulated cell }}}{\left(\frac{M F I_{\text {plasma membrane }}}{M F I_{\text {cytosol }}}\right)_{\text {resting cell }}}$

\subsubsection{Biochemical methods}

\subsubsection{Expression and purification of recombinant GST fusion proteins}

To yield fusion proteins with the GST moiety at the N-terminus and the protein of interest at the C-terminus the pGEX vector was transformed in E.coli BL21(DE3). A single colony was inoculated in 2YT/Amp medium and grown over night. The culture was diluted into $20-500 \mathrm{~mL}$ 2YT/Amp and grown until an $\mathrm{OD}_{600}$ of 0.6-0.8 was reached. Expression was induced with $0.1 \mathrm{mM}$ IPTG. After shaking at $37^{\circ} \mathrm{C}$ for $3 \mathrm{~h}$ bacteria were harvested by centrifugation at $3,000 \mathrm{xg}$ and $4^{\circ} \mathrm{C}$ for $20 \mathrm{~min}$. Bacterial pellets were resuspended in $1 / 20$ of the culture volume bacterial lysis buffer without Triton X-100 and the suspension sonicated six times for $15 \mathrm{~s}$. Triton X-100 was added to the lysate to a final concentration of $1 \%$ and incubated for $30 \mathrm{~min}$ on ice. The lysate was centrifuged at $6000 \mathrm{xg}$ at $4^{\circ} \mathrm{C}$ for $15 \mathrm{~min}$. The supernatant was transferred into a new reaction tube and incubated with 1/1000 of the culture volume glutathione sepharose 4 Fast flow (50\% slurry, GeHealthcare) rotating for $1 \mathrm{~h}$ or overnight at $4^{\circ} \mathrm{C}$. The sepharose beads were washed three times with Triton X-100 containing bacterial lysis buffer and either used for affinity purification experiments or eluted with GST elution buffer for immunisation of rabbits (expression method).

2YT medium

$16 \mathrm{~g} / \mathrm{L}$ select peptone; $10 \mathrm{~g} / \mathrm{L}$ yeast extract; $5 \mathrm{~g} / \mathrm{L} \mathrm{NaCl}$

Bacterial lysis buffer

50mM Tris/HCl, pH 7.4; 150mM NaCl; 5mM DTT; protease inhibitor

GST elution buffer

50mM Tris/HCl, $\mathrm{pH} 8 ; 10 \mathrm{mM}$ glutathione (reduced)

\subsubsection{Immunisation of rabbits with GST fusion proteins}

To produce polyclonal antiserum against chicken CD2AP the GST fusion protein chicken CD2AP_Ab_long was purified with glutathione sepharose and eluted with GST elution buffer. For the primary immunisation $75-100 \mu \mathrm{g}$ of the immunogenic protein was emulsified with $2 \mathrm{x}$ volumes Freund's complete adjuvant (final volume $300 \mu \mathrm{l}$ ) by sonification. The emulsion was injected subcutaneously in female Chinchilla Bastard rabbits (Charles River Laboratories, Germany) by a veterinarian at the Central Animal Facility University of Göttingen. At the date 
of primary injection $1 \mathrm{~mL}$ pre-immune serum was taken from the animals. The immune system of the animals was boosted three times every 3-4 weeks with an emulsion containing the immunogen in Freund's incomplete adjuvant. Then $50 \mathrm{~mL}$ blood were collected and centrifuged after coagulation at $1500 \times \mathrm{xg}$ and $4^{\circ} \mathrm{C}$ for $25 \mathrm{~min}$. The serum was transferred in a new tube and stored at $-20^{\circ} \mathrm{C}$. The antibody was tested on cleared cellular lysates from DT40 wild-type or cd2ap ${ }^{-1}$ DT40 B cells and used in a 1:2000 dilution.

All procedures were displayed in accordance with $\S 10$ a of the protection of animals act.

\subsubsection{Expression and purification of recombinant $\mathrm{His}_{6}$ fusion proteins}

To express human SLP65 with an N-terminal $\mathrm{His}_{6}$-tag the pET15b expression vector was transformed in E.coli BL21(DE3). A single colony was inoculated in LB/Amp and grown for 4h before dilution into $50 \mathrm{ml}$ minimal medium containing ampicillin and shaking overnight. The next day the overnight culture was transferred in $500 \mathrm{ml}$ minimal medium/Amp and grown until an $\mathrm{OD}_{600}$ of 0.6 . Expression was induced with $1 \mathrm{mM} I P T G$ and stopped after shaking for $3 \mathrm{~h}$ at $37^{\circ} \mathrm{C}$. Bacteria were harvested by centrifugation at $6000 \mathrm{rpm}$ and $4^{\circ} \mathrm{C}$ for $35 \mathrm{~min}$. Bacteria pellets were resuspended in $6 \mathrm{ml}$ LEW-buffer and lysed by sonification 3 times for 30s. After sonification Triton-X 100 was added to a final concentration of $0.25 \%$ and incubated on ice for $30 \mathrm{~min}$. Lysates were centrifuged at $18000 \mathrm{~g}$ and $4^{\circ} \mathrm{C}$ for $30 \mathrm{~min}$. The cleared lysate was run over an LEW equilibrated Ni-IDA column and the column washed with LEW buffer containing $5 \mathrm{mM}$ Imidazole. The recombinant protein was eluted off the column with $1.5 \mathrm{ml}$ elution buffer. LEW and elution buffers as well as Ni-NDA columns were taken from the Protino ${ }^{\circledR}$ Ni IDA 1000 kit, MachereyNagel.

Minimal medium

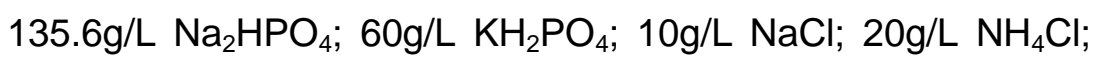
$0.4 \%$ glucose; $2 \mathrm{mM} \mathrm{MgSO}$; $0.1 \mathrm{mM} \mathrm{CaCl} ; 6 \mathrm{ml}$ thiamine hydrochloride $(5 \mathrm{mg} / \mathrm{mL}) ; 50 \mu \mathrm{g} / \mathrm{ml}$ ampicillin

\subsubsection{Stimulation of DT40 B cells via the BCR}

DT40 B cells were harvested and washed once with PBS. Cell were resuspended in RPMI 1640 without supplements and incubated at $37^{\circ} \mathrm{C}$ for $10-20 \mathrm{~min}$. Stimulation via the BCR was induced by adding $2 \mu \mathrm{g} / \mathrm{mL} \mathrm{M} 4$ to the cell suspension and vortexing. Cells were incubated for desired time points and directly centrifuged at $950 \mathrm{xg}$ and at $4^{\circ} \mathrm{C}$ for $2 \mathrm{~min}$. Cells were lysed by adding $20 \mu \mathrm{l} / 1 \cdot 10^{6}$ cell lysis buffer and incubation for $30 \mathrm{~min}$ on ice. Lysates were centrifuged at maximum speed and $4^{\circ} \mathrm{C}$ for $10 \mathrm{~min}$. The cleared cellular lysate (CCL) was either mixed 
with $1 / 4$ 4XSDS sample buffer and heated at $95^{\circ} \mathrm{C}$ for $5 \mathrm{~min}$ or subjected to immuno- or affinity purification experiments.

Lysis buffer

50mM Tris/HCl, pH 8; $150 \mathrm{mM} \mathrm{NaCl;} 5 \mathrm{mM} \mathrm{NaF} ; 1 \mathrm{mM} \mathrm{Na}_{3} \mathrm{VO}_{4}$; protease inhibitor cocktail; $0.5 \% \mathrm{NP}-40$

4xSDS sample buffer

250mM Tris/HCl, pH 6.8; 200mM DTT; 40\% glycerol; 8\% SDS;

$0.05 \%$ bromophenol blue

\subsubsection{Affinity purification with GST fusion proteins or antibodies}

For affinity purifications with GST fusion proteins cleared cellular lysates from $2-5 \cdot 10^{7}$ cells were incubated with $10 \mu \mathrm{g}$ of the fusion protein immobilised on glutathione sepharose rotating at $4^{\circ} \mathrm{C}$ overnight. Alternatively CCLs were incubated with $0.5-2 \mu \mathrm{g}$ of the precipitating antibody overnight and immobilised on $15 \mu \mathrm{l}$ Protein $\mathrm{A} / \mathrm{G}$ plus agarose (Santa Cruz Biotechnology, Inc.) incubated for $45 \mathrm{~min}$. For precipitation of the BCR, DT40 B cells were stimulated with $2 \mu \mathrm{g} / \mathrm{mL}$ M4 (anti-chicken $\operatorname{lgM}$, mouse $\operatorname{lgM}$ ) at $37^{\circ} \mathrm{C}$ or on ice (0 min time point). Cells were washed once with PBS to remove residual unbound M4 before resuspension in lysis buffer. After rotating over night the lysate was incubated with $2 \mu \mathrm{g}$ goat anti-mouse $\lg \mathrm{M}$ for $1 \mathrm{~h}$ to allow subsequent immobilisation with ProteinA/G Plus agarose. The beads were washed two times with $600 \mu$ lysis buffer and were finally heated in $50 \mu \mathrm{l} 4 \mathrm{x}$ SDS sample buffer at $95^{\circ} \mathrm{C}$ for $5 \mathrm{~min}$. Purified proteins were resolved by SDS-PAGE.

For analysis of in vitro interaction of recombinant $\mathrm{His}_{6}$-SLP65 with GST-CIN85(SH3) $)_{3}$ coupled to glutathione sepharose $10 \mu \mathrm{g}$ of both proteins were incubated in NMR buffer and rotated for $2 \mathrm{~h}$ at $4^{\circ} \mathrm{C}$. Beads were washed three times with $1 \mathrm{~mL}$ NMR buffer and heated in $4 \times \mathrm{SDS}$ sample buffer before analysis.

NMR buffer

$20 \mathrm{mM}$ Bis-Tris; $150 \mathrm{mM} \mathrm{KCl;} \mathrm{2mM} \mathrm{DTT;} 0.01 \% \mathrm{NaN}_{3}$; $\mathrm{pH} 7$

\subsubsection{SDS-polyacrylamide gel electrophoresis (SDS-PAGE) (Laemmli 1970)}

For the separation of proteins by electrophoresis the discontinuous SDS gel system was used. Proteins in a sample are concentrated in the stacking and resolved by molecular weight in the separating gel. Electrophoresis was performed at $12 \mathrm{~mA}$ for the stacking and 50mA for the separating gel in a Biorad gel electrophoresis system. As apparent molecular weight standard the prestained protein marker (NEB) was used. For the visualisation of proteins SDS-gels were either stained with Coomassie dye or subjected to western blot analysis. 
Stacking gel

Separating gel

SDS running buffer

Coomassie staining solution 2.5g/L Coomassie Brilliant Blue R250; 45\% MeOH; 10\% acetic acid

\subsubsection{Western blot analysis}

After SDS-PAGE proteins were transferred onto nitrocellulose membranes via semi-dry blotting. A sheet of transfer buffer soaked whatman paper was put on the anode followed by the nitrocellulose membrane, the SDS gel, a second whatman paper and the cathode. The transfer was performed applying a constant voltage of $16 \mathrm{~V}$ for $1 \mathrm{~h}$. The membrane was put in blocking solution for $1 \mathrm{~h}$ at RT. For the specific detection of proteins the membrane was incubated with the primary antibody diluted in antibody solution. For detection via chemiluminescence an HRPO-coupled secondary antibody was used in a 1:10,000 dilution. The immunostaining was visualised using the ECL solution and a digital imaging system (Chemi Lux , Intas).

Transfer buffer

39mM glycine; 48mM Tris; $0.0375 \%$ (w/v) SDS; $0.01 \%$ (w/v) $\mathrm{NaN}_{3}$; $20 \% \mathrm{MeOH}$

Blocking solution $\quad 5 \%(\mathrm{w} / \mathrm{v}) \mathrm{BSA}$ in TBS-T

ECL solution

$4 \mathrm{ml}$ solution A was freshly mixed with $400 \mu$ l solution $\mathrm{B}$ and $1.2 \mu \mathrm{I}_{2} \mathrm{O}_{2}$

Solution A $\quad 250 \mathrm{mg} / \mathrm{L}$ luminol; $0.1 \mathrm{M} \mathrm{Tris} / \mathrm{HCl}, \mathrm{pH} 8.6,4^{\circ} \mathrm{C}$

Solution B $\quad 55 \mathrm{mg}$ para-Coumaric acid/50mL DMSO 


\section{Results}

\subsection{CIN85 is a positive regulator in the onset of $\mathrm{BCR}$-induced $\mathrm{Ca}^{2+}$ signalling, but can be replaced by CD2AP}

\subsubsection{CIN85 has a positive regulatory role in $\mathrm{BCR}$-induced $\mathrm{Ca}^{2+}$ flux}

In order to analyse the impact of the preformed SLP65 and CIN85 and/or CD2AP complex on BCR-induced signal transduction from the side of CD2AP and CIN85, first, a cin85 DT40 B cell line was generated (Dr.K Neumann). Due to the existence of multiple promoters in the rat cin85 (ruk) gene targeting the 5 region of the chicken cin85 gene still showed expression of CIN85 isoforms (data not shown). Therefore, I decided to use RNA interference (RNAi) to explore the function of CIN85 in DT40 B cells. cin85 gene expression was arrested using retroviral vectors encoding a short hairpin (shRNA) targeting the $3^{\prime}$ untranslated region (UTR) of chicken cin85 mRNA. An unspecific shRNA (mock) was always carried along to rule out any effects derived from the blockage of the endogenous RNAi pathway by overexpression of a synthetic shRNA. In the following sections cells that were infected with shRNA against $\operatorname{cin} 85$ mRNA or an unspecific shRNA are referred to as $\operatorname{cin} 85^{\text {sh }}$ or mock ${ }^{\text {sh }}$ DT40 B cells, respectively.

To estimate the expression of chicken cin85 and shRNA-induced changes, DT40 B cells were infected with shRNA against cin85 mRNA or control shRNA and cleared cellular lysates were analysed by immunoblot analysis (figure 3.1). Immunostaining with anti-CIN85 antibodies detected two signals at about 160 kilo Dalton $(\mathrm{kDa})$ and two at $80 \mathrm{kDa}$. The signals at $160 \mathrm{kDa}$ are not targeted by the shRNA and are possibly unspecifically detected by the anti-CIN85 antibody. The upper signal at $80 \mathrm{kDa}$ is considered to be the long CIN85 isoform comprising all three $\mathrm{N}$-terminal $\mathrm{SH} 3$ domains, while the lower is a truncation variant that lacks the first $\mathrm{SH} 3$ domain. In mouse tissues these isoforms are referred to as Ruk and Ruk $_{\triangle A}$, respectively (Buchman et al. 2002). The CIN85 signal of both isoforms shows is strongly reduced in cells expressing the shRNA against cin 85 mRNA (cin85 ${ }^{\text {sh }}$ ). Thus, the cin85 ${ }^{\text {sh }}$ DT40 B cells could be used to analyse the function of CIN85 in BCR signal transduction. 


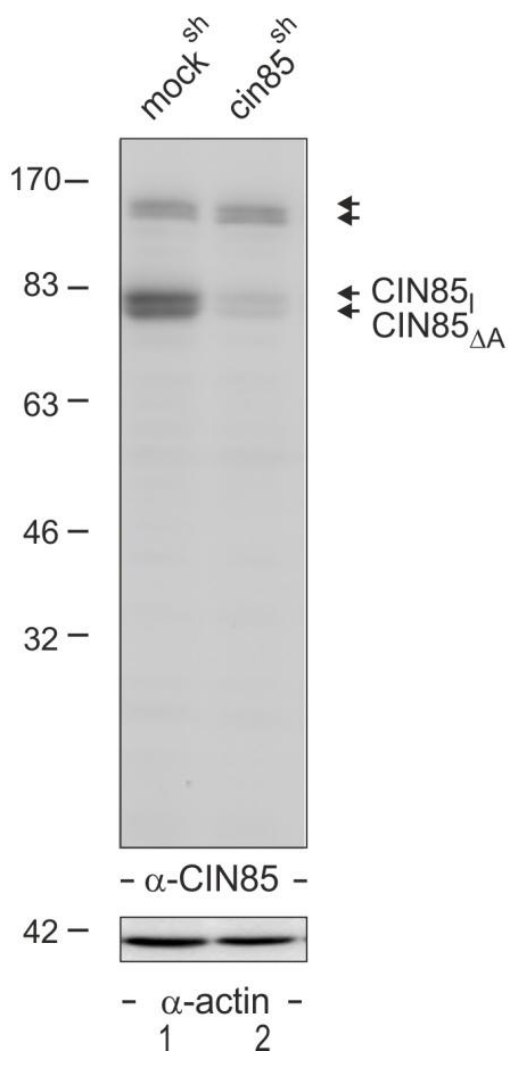

Figure 3.1: Both CIN85 isoforms at $80 \mathrm{kDa}$ are targeted by the shRNA in DT40 B cells.

Wild-type DT40 B cells expressing an shRNA against cin85 mRNA ( $\operatorname{cin} 85^{\text {sh }}$ ) or a control shRNA $\left(\right.$ mock $\left.^{\text {sh }}\right)$ were analysed by immunoblotting with anti-CIN85 or anti-actin antibodies (upper and lower panel, respectively). Arrows indicate the signals detected by the anti-CIN85 antibody. The two CIN85 isoforms at $80 \mathrm{kDa}$ were termed CIN85, and CIN85 ${ }_{\triangle A}$ corresponding to the Ruk and Ruk ${ }_{\triangle A}$ isoforms in mouse tissues (Buchman et al. 2002).

For BCR-induced $\mathrm{Ca}^{2+}$ mobilisation analysis of mock $^{\text {sh }}$ and $\operatorname{cin} 85^{\text {sh }}$ DT40 B cells, the amount of CIN85 was always controlled in parallel. Figure 3.2A shows a considerable reduction of cin85 expression in cin85 ${ }^{\text {sh }}$ DT40 B cells. The shRNA-infected cells were subjected to BCRinduced $\mathrm{Ca}^{2+}$ mobilisation analysis using high or low concentrations of stimulating antibodies (figure $3.2 \mathrm{~B}$ and $\mathrm{C}$, respectively). When stimulated with $0.2 \mu \mathrm{g} / \mathrm{ml}$ both mock $^{\text {sh }}$ and ${\text { cin } 85^{\text {sh }}}^{\text {sh }}$ wild-type DT40 B cells responded with a fast increase followed by a slow decline of intracellular $\mathrm{Ca}^{2+}$ concentration (figure 3.2B). After low BCR stimulus comparison of the $\mathrm{Ca}^{2+}$ profiles of mock ${ }^{\text {sh }}$ and cin85 $5^{\text {sh }}$ wild-type DT40 B cells (figure 3.2B and C, blue and red line, respectively) revealed that reduced expression of cin85 led to a slightly, but significantly decreased and delayed $\mathrm{Ca}^{2+}$ response. Thus, CIN85 exerts a positive function in the kinetic and magnitude of the $\mathrm{Ca}^{2+}$ signal after $\mathrm{BCR}$ cross-linking with low amounts of stimulating antibody. 
A) B)
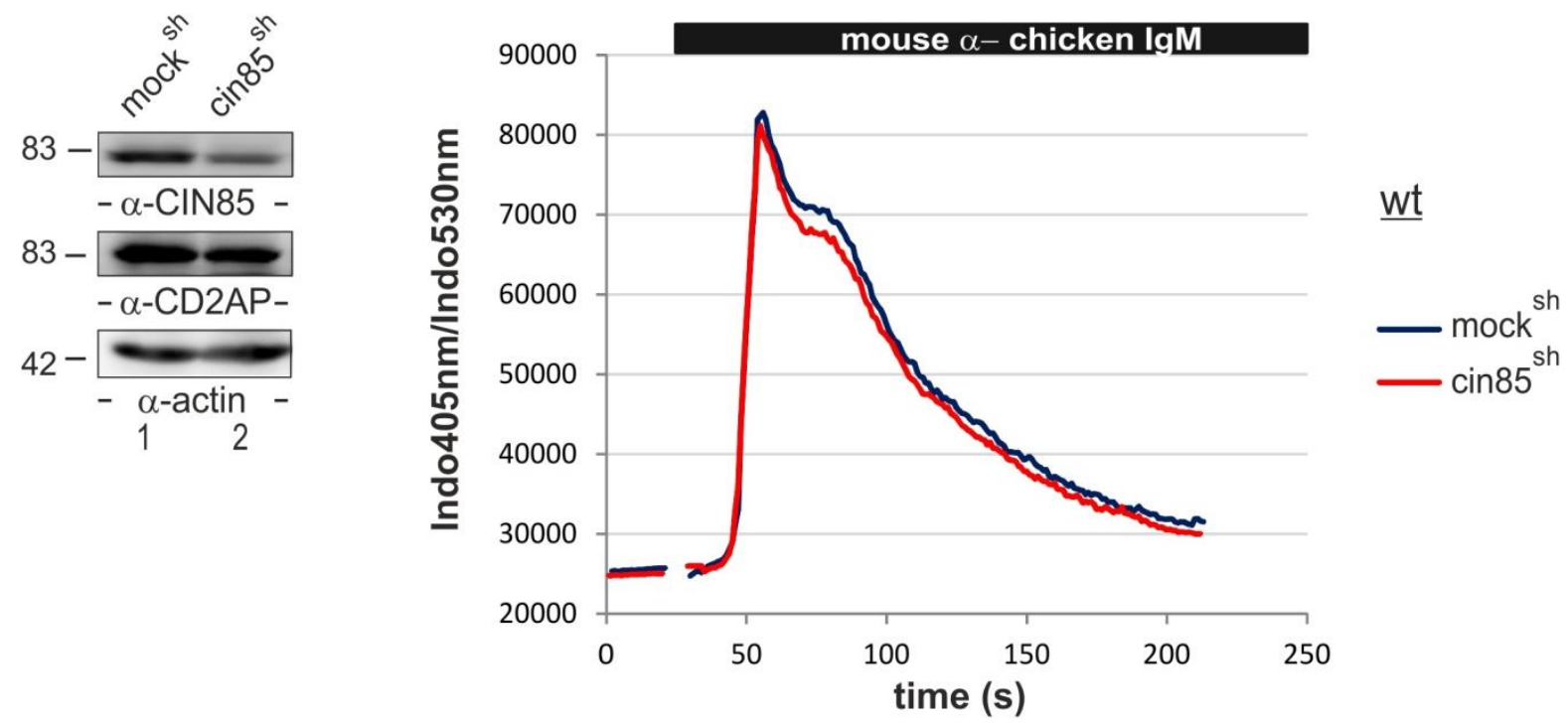

C)

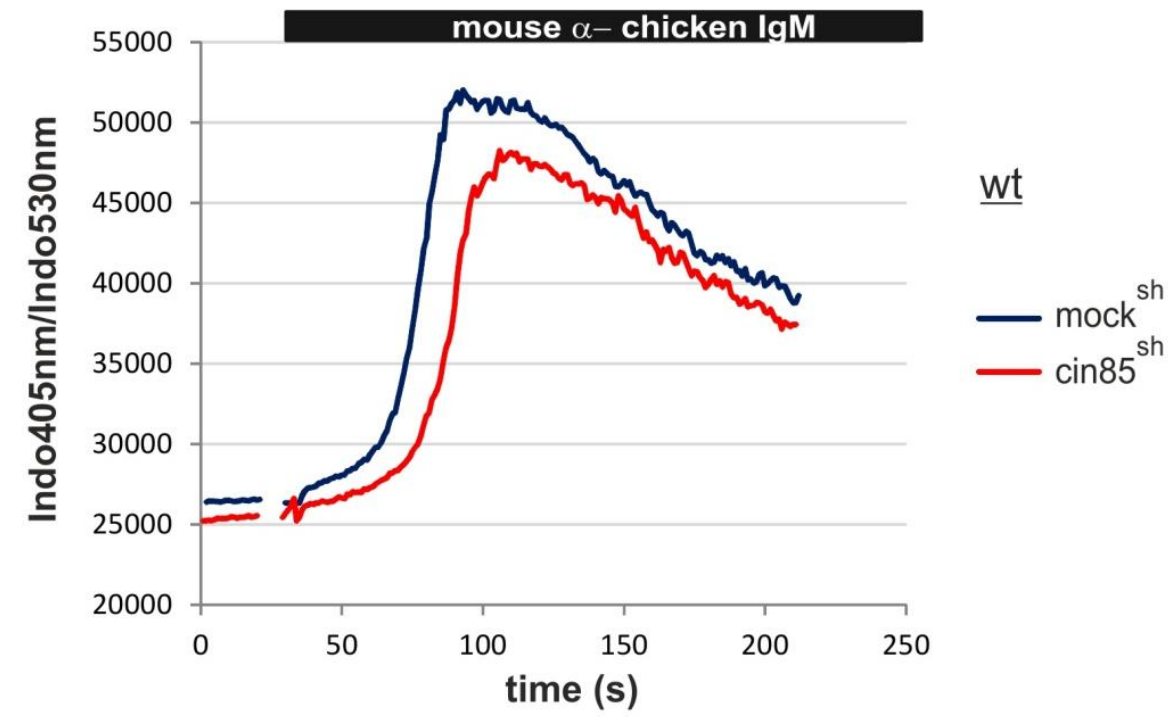

Figure 3.2: $\mathrm{CIN85}$ is a positive regulator of $\mathrm{BCR}$-induced $\mathrm{Ca}^{2+}$ flux.

A) Wild-type DT40 B cells expressing an shRNA against $\operatorname{cin} 85$ mRNA (cin85 ${ }^{\text {sh }}$ ) or a control shRNA $\left(\right.$ mock $^{\text {sh }}$ ) were either subjected to immunoblot analysis using anti-CIN85, anti-chicken CD2AP or antiactin antibodies (upper, middle and lower panel, respectively) or B) and C) to flow cytometry for BCRinduced $\mathrm{Ca}^{2+}$ flux analysis using either $0.2 \mu \mathrm{g} / \mathrm{ml}$ or $0.02 \mu \mathrm{g} / \mathrm{ml}$ anti-chicken $\mathrm{lgM}$ antibodies (B and $\mathrm{C}$, respectively). Apparent molecular weights of protein standards are indicated on the left in $\mathrm{kDa}$. Parts of this figure are published in (Oellerich et al. 2011).

\subsubsection{CD2AP is dispensable for $\mathrm{BCR}$-induced $\mathrm{Ca}^{2+}$ mobilisation}

To elucidate a contribution of CD2AP to B lymphocyte signalling a cd2ap ${ }^{\text {- }}$ chicken DT40 B cell line was generated by homologous recombination. For the design of targeting vectors the chicken cd2ap messenger ribonucleic acid (mRNA) (GenBank entry AJ720324.1) was 
blasted against the chicken genome (http://genome.ucsc.edu/cgi-bin/hgBlat) and the cd2ap gene locus was mapped to chromosome 3:112824723-112889577.

A)
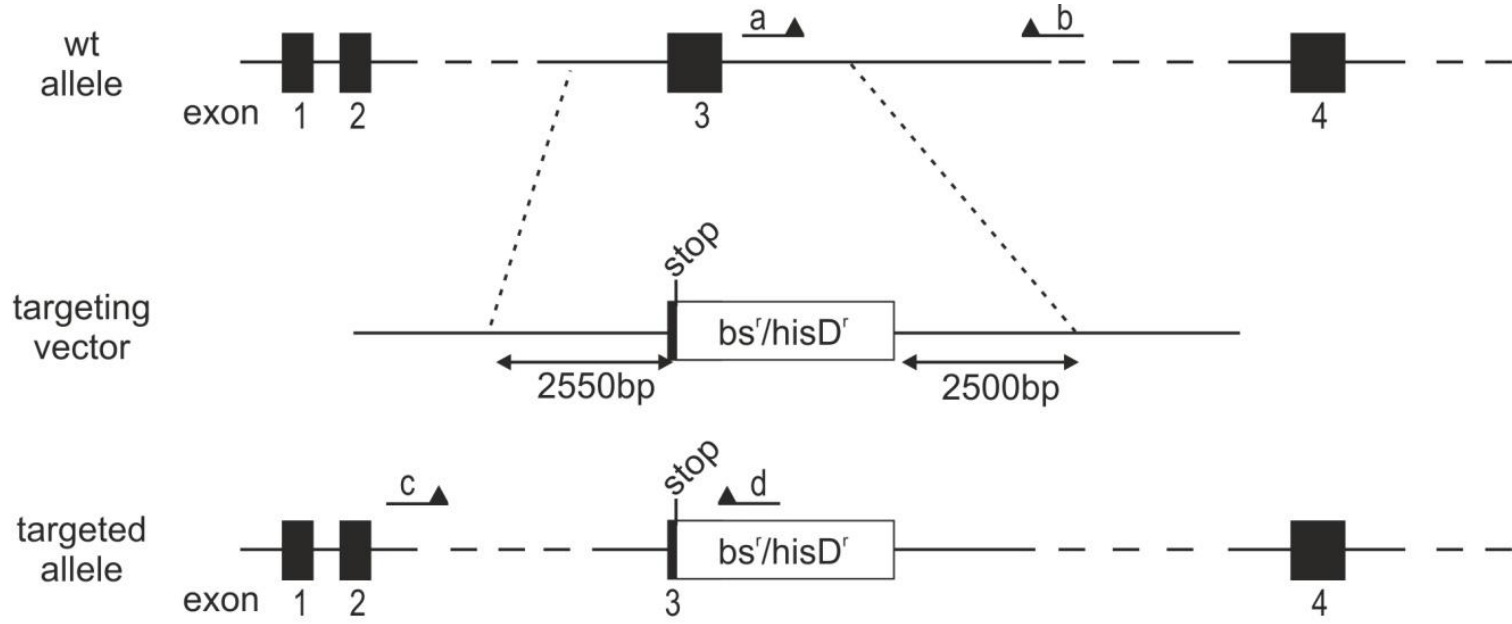

B)

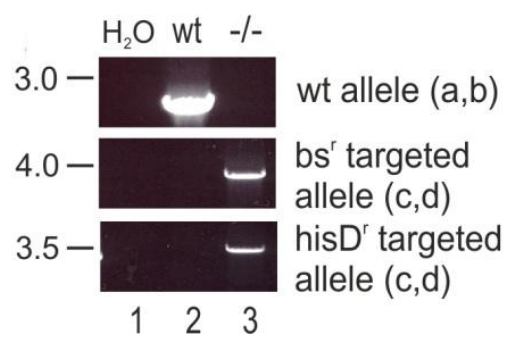

C)

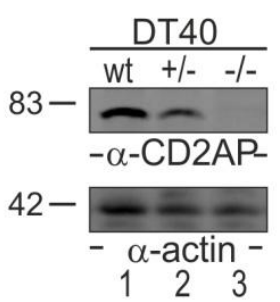

Figure 3.3: Generation of a cd2ap ${ }^{-/}$DT40 B cell line.

A) Schematic representation of the chicken cd2ap wild-type allele, the targeting vector and the targeted allele. The genomic locus of chicken cd2ap on chromosome 3 (wt allele) was targeted using the vectors pBluescript-cd2ap-LARA-bsr or pBluescript-cd2ap-LARA-HisD either coding for a blasticidin $S$ or a histidinol D resistance cassette $\left(\mathrm{bs}^{r}\right.$ or his $\mathrm{D}^{r}$, respectively), $3^{\prime}$ and $5^{\prime}$ flanked by genomic regions (targeting vector, left arm 2550bp, right arm 2500bp). The resistance cassettes were inversely $\left(3^{\prime} \rightarrow 5^{\prime}\right)$ integrated into the targeting vector. Homologous recombination of the targeting constructs into the genomic locus resulted in an introduction of a stop codon $3^{\prime}$ of the third codon in exon 3 and replacement of the downstream 2000bp by either bs $^{r}$ or hisD ${ }^{r}$ (targeted allele). Depicted sizes and distances are not drawn to scale. a,b,c,d: oligonucleotides used for genotype analysis, see B). The targeting strategy was designed by Dr. K. Neumann. B) Genomic DNA of wild-type (lane 2) or cd2ap $^{-1}$ (lane 3) DT40 B cells was analysed for targeted integration of the $\mathrm{bs}^{\mathrm{r}}$ or hisD ${ }^{\mathrm{r}}$ or the wt allele via PCR using primers $c$ and $d$ or $a$ and $b$, respectively. A PCR reaction without genomic DNA was performed as a negative control $\left(\mathrm{H}_{2} \mathrm{O}\right.$, lane 1). Primers: a: wtscreen; b: RAscreen; c: LAscreen, $d$ : either his (lower panel) or blast-300 (middle panel). C) Cleared cellular lysates from wild-type (lane 1), $\mathrm{cd} \mathrm{ap}^{+/}$(lane 2) or cd2ap ${ }^{-/}$(lane 3) DT40 B cells were subjected to SDS-PAGE and analysed on an immunoblot with antibodies recognising chicken CD2AP (upper panel) or actin (lower panel). Apparent molecular weights of protein or DNA standards are indicated on the left in $\mathrm{kDa}$ or $\mathrm{kb}$, respectively. For all experiments shown $c d 2 a p^{\%}$ clone 7.17 was used. 
Two targeting vectors were created that encode either a blasticidin S (bs) or a histidinol D (hisD) resistance cassette, $3^{\prime}$ and $5^{`}$ flanked by genomic regions of the cd2ap gene locus. Targeted integration resulted in a 2 kilo base $(\mathrm{kb})$ gene deletion and the introduction of an in-

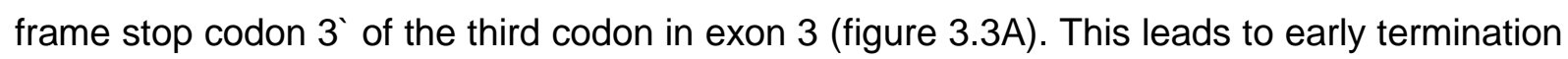
of remaining transcripts. Targeted integration was analysed by PCR using genomic DNA from wild-type or cd2ap $\%$ DT40 B cells (figure 3.3B) and on the protein level by immunoblotting with anti-CD2AP antibodies (figure 3.3C). While wild-type DT40 B cells showed expression of CD2AP, it was reduced in heterozygous cd2ap ${ }^{+/}$and not detectable in cd2ap $^{-/}$DT40 B cells.

Next, cd2ap ״DT40 B cells were retrovirally transduced to express either Citrine-tagged wildtype CD2AP or enhanced Green Fluorescent Protein (EGFP) alone as a negative control (figure 3.4, wt or cd2ap ${ }^{-/}$, respectively) and their competence to mobilise $\mathrm{Ca}^{2+}$ was analysed by flow cytometry. Upon stimulation of the BCR cd2ap ${ }^{\%}$ and cd2ap ${ }^{\%}$ DT40 B cells reconstituted with CD2AP showed no difference in their $\mathrm{Ca}^{2+}$ flux (figure 3.4B and C). 
A)

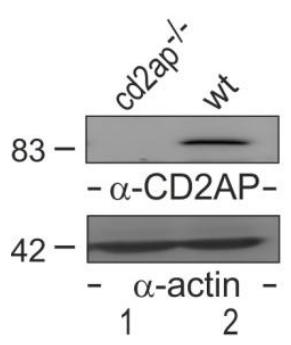

B)

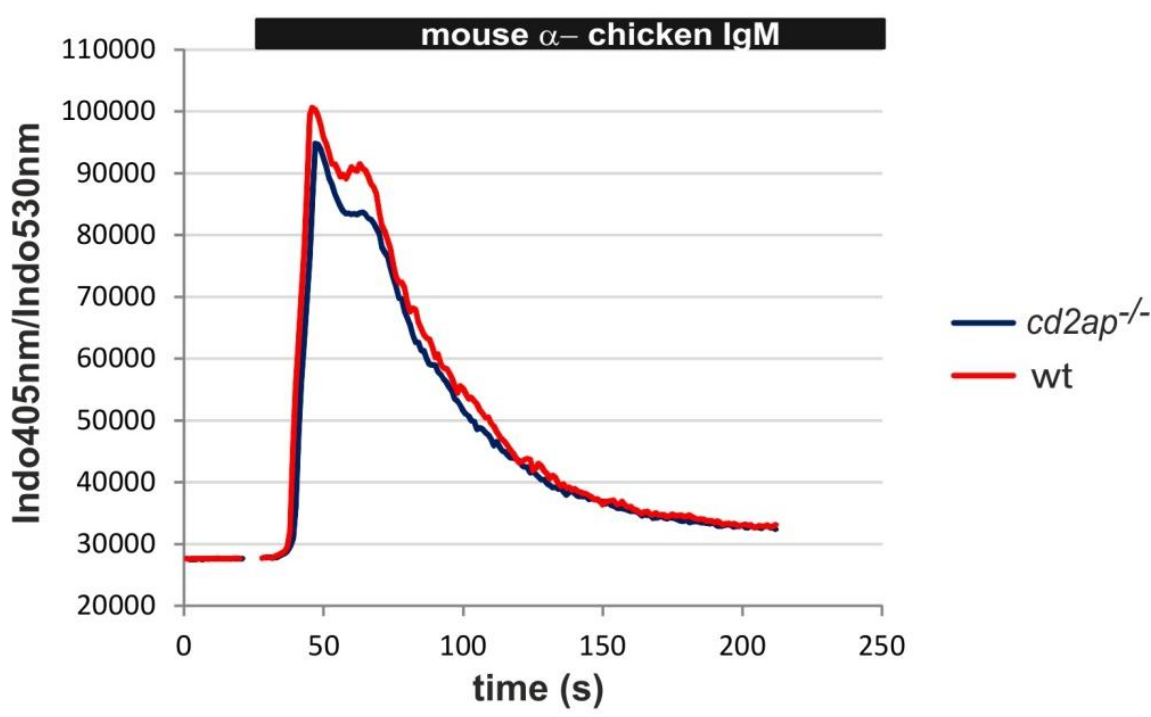

C)

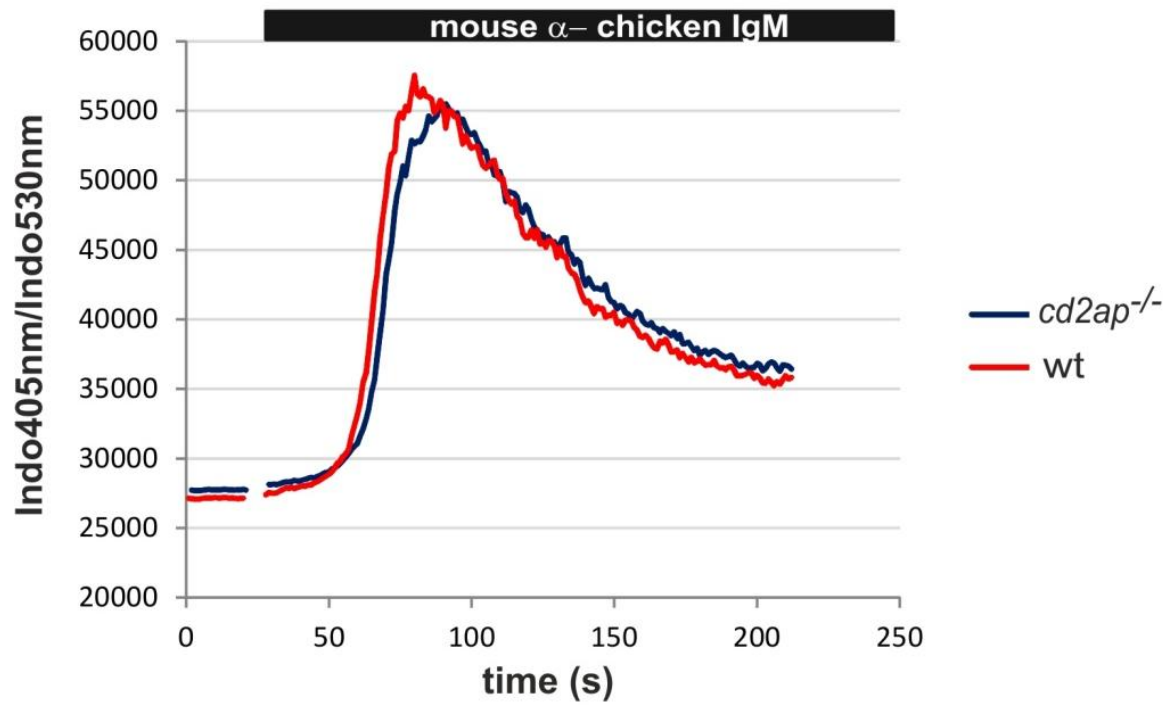

Figure 3.4: $\mathrm{CD2AP}$ is dispensable for $\mathrm{BCR}$-induced $\mathrm{Ca}^{2+}$ mobilisation.

A) CD2AP-deficient DT40 B cells retrovirally transduced with a vector coding for Citrine-tagged chicken CD2AP (wt) or for EGFP as a control $\left(\mathrm{cd}_{2} \mathrm{ap}^{-/}\right)$were either analysed by immunoblotting with anti-chicken CD2AP or anti-actin antibodies (upper and lower panel, respectively) or B) and C) subjected to flow cytometry for BCR-induced $\mathrm{Ca}^{2+}$ flux analysis using either $0.2 \mu \mathrm{g} / \mathrm{ml}$ or $0.02 \mu \mathrm{g} / \mathrm{ml}$ anti-chicken IgM antibodies ( $\mathrm{B}$ and $\mathrm{C}$, respectively). Apparent molecular weights of protein standards are indicated on the left in kDa. Parts of this figure are published in (Oellerich et al. 2011)

\subsubsection{Reduced expression of cin85 combined with CD2AP-deficiency impaired BCR- induced $\mathrm{Ca}^{2+}$ mobilisation}

The data presented so far pointed to a function of CIN85 rather than CD2AP in BCR-induced $\mathrm{Ca}^{2+}$ signalling. The effect of cin85 expression arrest was small compared to the SLP65 variant unable to bind CIN85 or CD2AP. Since both, CIN85 and CD2AP were identified as 
interaction partners of SLP65 and both show the same overall domain architecture as well as high sequence homology (Dikic 2002), I wanted to test if there was a functional redundancy of the two in $\mathrm{BCR}$-induced $\mathrm{Ca}^{2+}$ mobilisation.
A)
B)
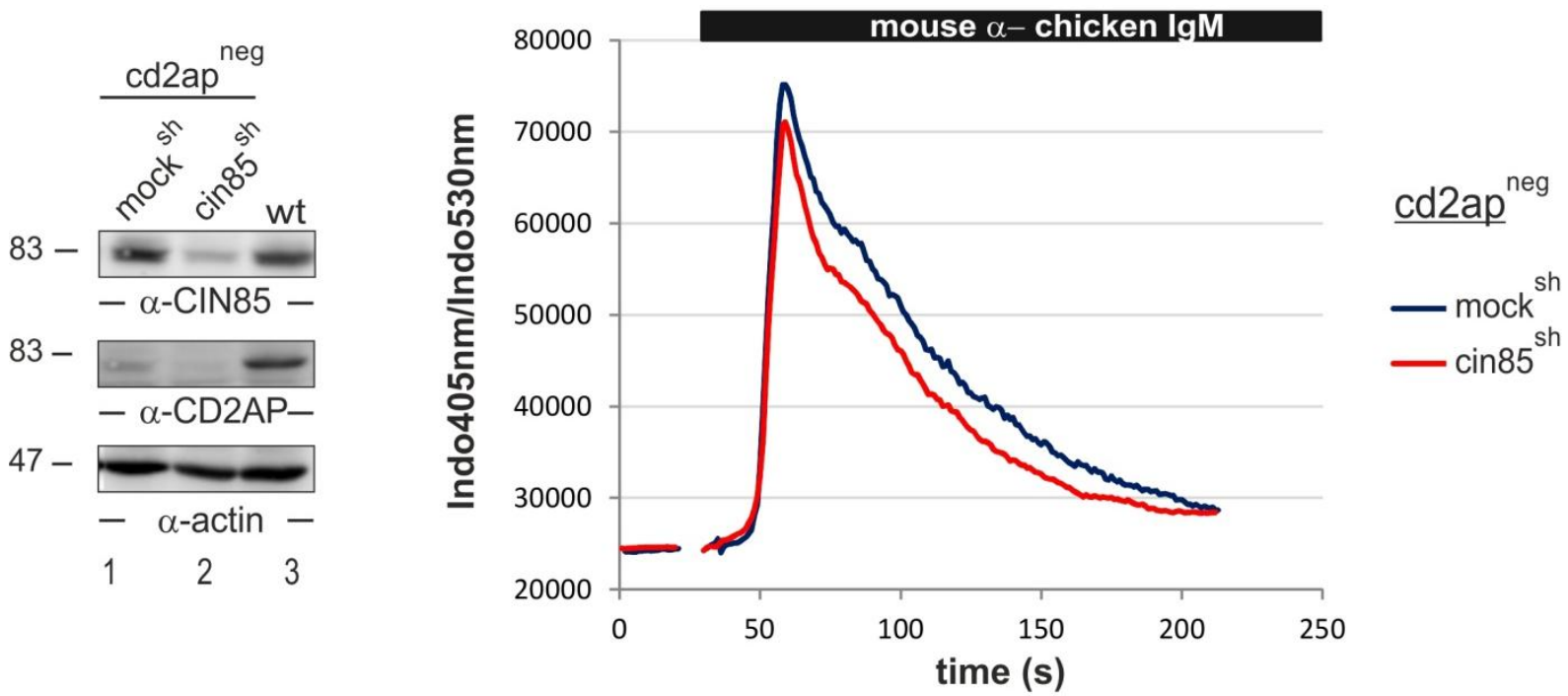

C)

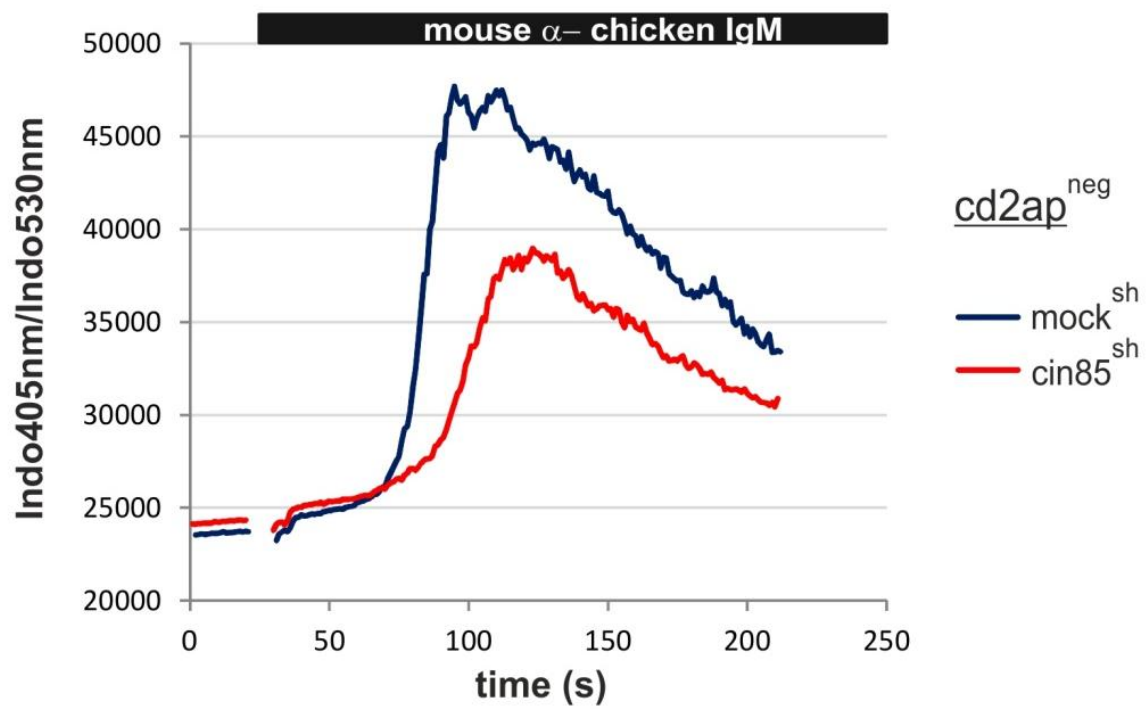

Figure 3.5: $\mathrm{BCR}$-induced $\mathrm{Ca}^{2+}$ mobilisation is impaired upon reduced cin85 expression and CD2AP-deficiency.

A) CD2AP-deficient DT40 B cells (cd2ap ${ }^{\text {neg }}$ ) expressing an shRNA against cin85 mRNA (cin85 ${ }^{\text {sh }}$ ) or a control shRNA (mock ${ }^{\text {sh }}$ ) were either subjected to immunoblot analysis using anti-CIN85, anti-chicken CD2AP or anti-actin antibodies (upper, middle and lower panel, respectively) or B) and C) to flow cytometry for BCR-induced $\mathrm{Ca}^{2+}$ flux analysis using either $0.2 \mu \mathrm{g} / \mathrm{ml}$ or $0.02 \mu \mathrm{g} / \mathrm{ml}$ anti-chicken $\mathrm{lgM}$ antibodies ( $\mathrm{B}$ and $\mathrm{C}$, respectively). Apparent molecular weights of protein standards are indicated on the left in kDa. Parts of this figure are published in (Oellerich et al. 2011). 
Therefore, I infected cd2ap ${ }^{-1}$ DT40 B cells with the constructs coding for the anti-cin85 or a control shRNA. Immunostaining of cleared cellular lysates from $c d 2 a p^{\text {neg }} / \mathrm{cin} 85^{\text {sh }}$ cells showed a strong reduction in CIN85 on the protein level compared to wild-type (wt) or cd2ap $^{\text {neg }} /$ mock $^{\text {sh }}$ cells (figure $3.5 A$ ). CD2AP was only detectable in wild-type DT40 B cells (figure 3.5A, lane 3 ) and equal loading was confirmed by anti-actin immunostaining. BCRinduced $\mathrm{Ca}^{2+}$ analysis showed a diminished and delayed response of $c d 2 a p^{\text {neg }} / \mathrm{cin} 85^{\text {sh }}$ cells compared to the $c d 2 a p^{\text {neg }} / \mathrm{mock}^{\text {sh }}$ control cells under high as well as low stimulating conditions (figure 3.5B and C). Again using low concentrations of cross-linking antibody the positive effects of $c d 2 a p$ and cin85 expression on a fast and strong $\mathrm{Ca}^{2+}$ response was more pronounced (see also figure $3.2 \mathrm{C}$ ).

In summary, CD2AP-deficiency alone had no influence on the $\mathrm{Ca}^{2+}$ profile, while reduced cin85 expression resulted in a moderate decrease and delay in $\mathrm{Ca}^{2+}$ flux after $\mathrm{BCR}$ activation. However, CD2AP could exert positive effects on $\mathrm{BCR}$-induced $\mathrm{Ca}^{2+}$ signalling when the physiological amount of CIN85 protein was reduced.

\subsubsection{Ectopic re-expression of cin85 reverses the defect in the onset of BCR-induced $\mathrm{Ca}^{2+}$ mobilisation in cin85 ${ }^{\text {sh }}$ DT40 B cells}

RNAi approaches have the disadvantage of giving false positive results, as the synthetic shRNA might also bind non-specifically to other mRNAs. Thus, I wanted to establish a system in which, after interference with cin85 expression by shRNAs, cin85 expression could be reconstituted by ectopically expressed cin85 complementary DNA (cDNA). The introduced cin85 cDNA was not targeted by the shRNA, since it did, in contrast to the endogenous cin85 mRNA, not contain the 3 UTR. Figure 3.6 gives an overview of the experimental steps undertaken: wild-type (wt) or CD2AP-deficient (cd2ap ${ }^{\text {neg }}$, not shown) DT40 B cells were retrovirally transduced to express the shRNA targeting cin85 mRNA or an unspecific shRNA and selected for stable integration of the construct (figure 3.6, 2). Subsequently, $\mathrm{wt} / \mathrm{cin} 85^{\text {sh }}$ or $\mathrm{cd} 2 \mathrm{ap} \mathrm{p}^{\text {neg }} / \mathrm{cin} 85^{\text {sh }}$ or their respective mock $^{\text {sh }}$ controls, were infected a second time with viruses carrying a vector encoding the cin85 cDNA fused to citrine. The cells were not selected for cDNA expression so that there were Citrine-positive as well as Citrine-negative cells in one culture (figure 3.6, 3, black or white part of the well, respectively). For subsequent $\mathrm{BCR}$-induced $\mathrm{Ca}^{2+}$ mobilisation analysis the Citrine-positive and Citrine-negative cells could be discriminated by concomitant flow cytometry. The latter cells served as an internal negative control (4). 


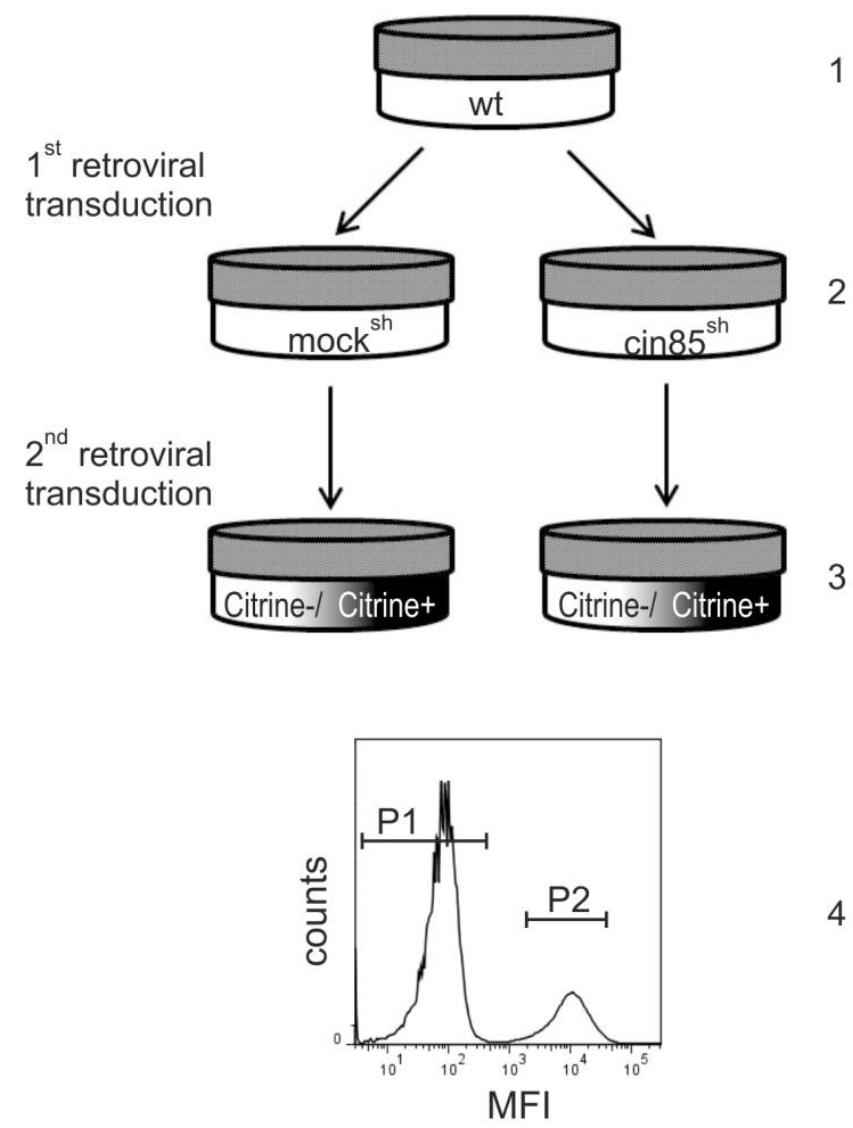

Figure 3.6: Experimental strategy for the reconstitution of cin85/cd2ap expression in shRNA transfected DT40 B cells.

Schematic drawing of the genetic modifications introduced to reconstitute cin85/cd2ap expression. Wild-type $(1, \mathrm{wt})$ or $c d 2 a p^{-1}$ DT40 B cells (not shown) were retrovirally transduced with vectors coding for an shRNA directed against the $3^{\prime}$ UTR of chicken cin85 mRNA (cin85 ${ }^{\text {sh }}$ ) or with an unspecific shRNA (mock ${ }^{\text {sh }}$ ) (2). After selection with the appropriate antibiotic and recovery of the cells they were transduced a $2^{\text {nd }}$ time with viruses containing the cDNA of interest in a Citrine-coding vector. After the infection, cells were not selected for expression of the construct resulting in a cell population that contains both, Citrine-positive (3, black part of the well, Citrine+) as well as Citrine-negative cells ( 3 , white part of the well, Citrine-) serving as an internal control. After flow cytometric analysis cells in 3 can be gated for Citrine-negative (P1) or Citrine-positive populations (P2) (4).

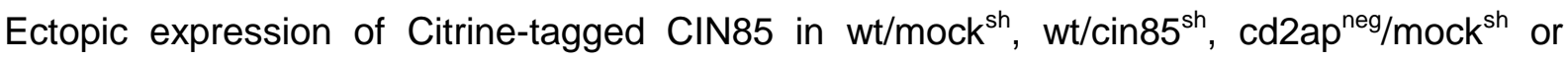
cd2ap $^{\text {neg }} / \mathrm{cin}^{\text {sh }}$ DT40 B cells was confirmed by immunoblot analysis with anti-CIN85 antibodies (figure 3.7A, upper panel). With the same immunostaining the reduction of endogenous cin85 expression could be determined in cin85 $5^{\text {sh }}$ cells (figure $3.7 \mathrm{~A}, 2^{\text {nd }}$ panel, lane 2 and 4). 
A)

B)
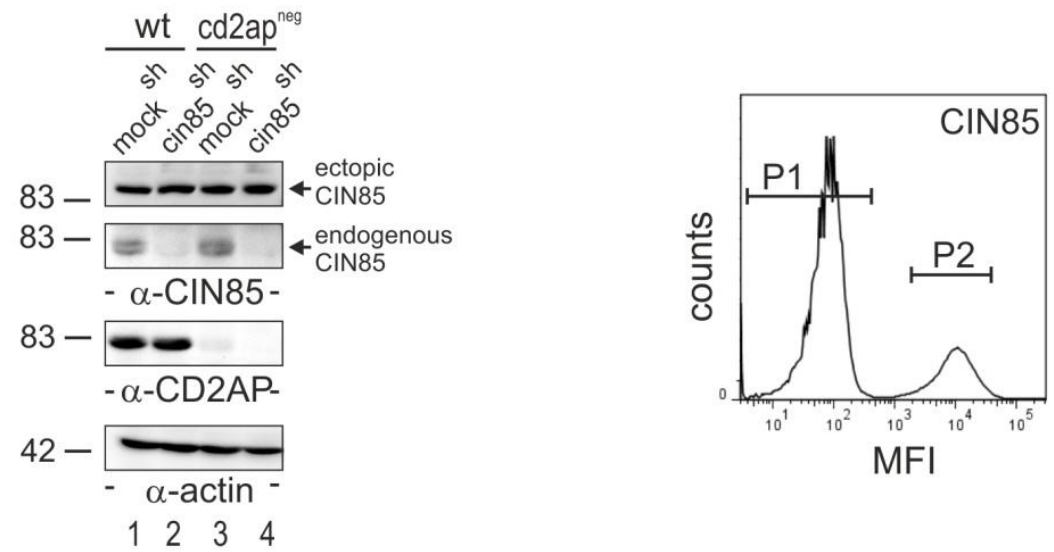

C)

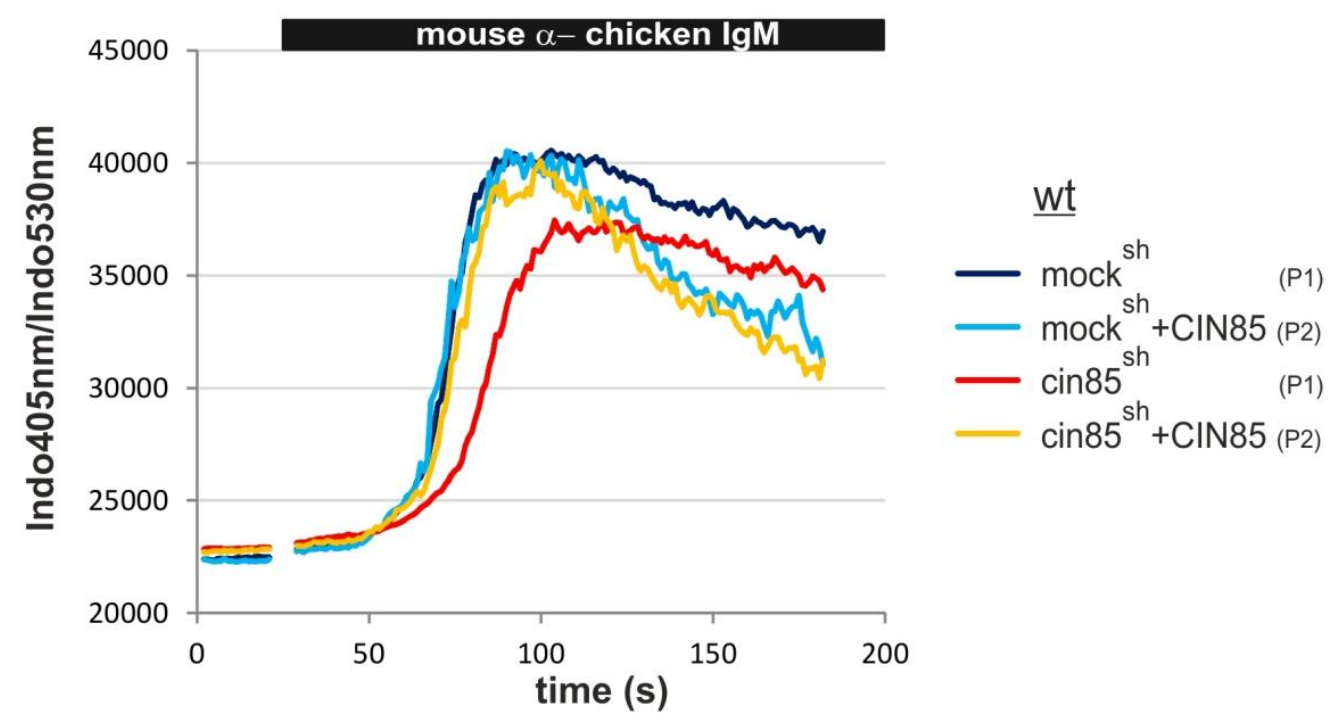

D)

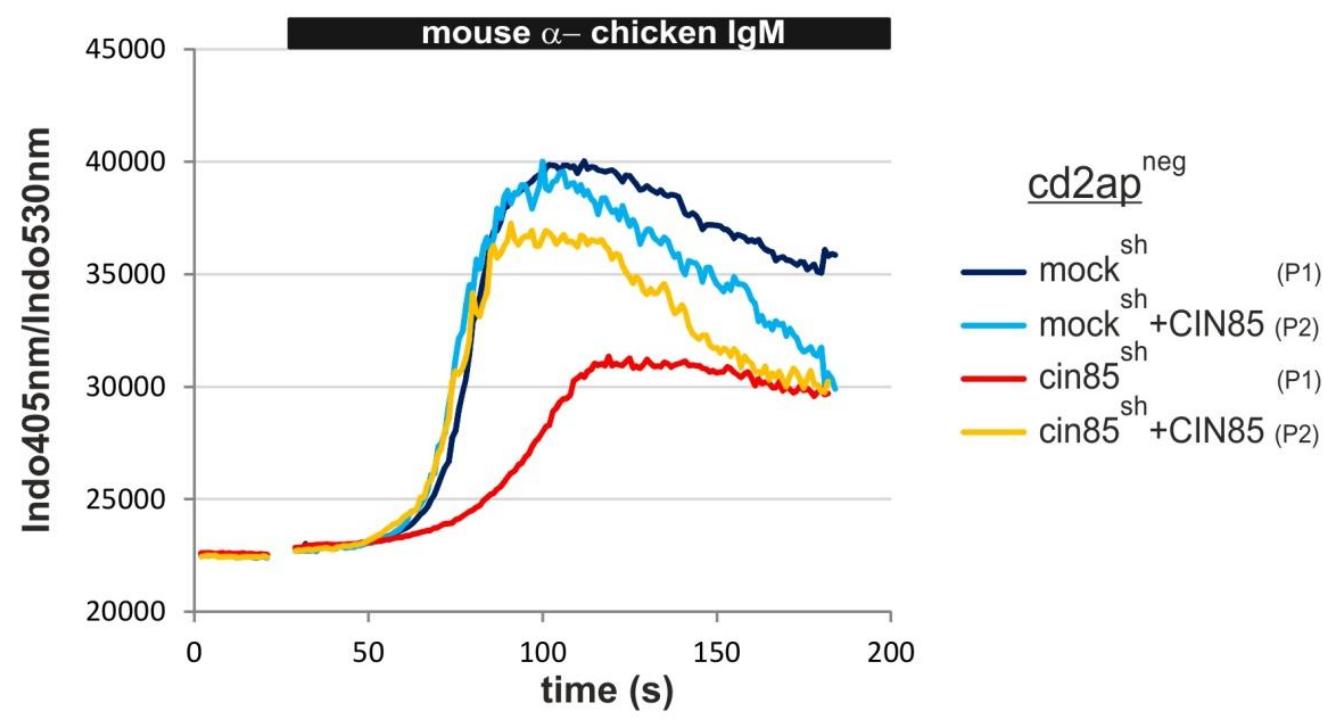

Figure 3.7: Ectopic re-expression of cin85 reverses the defect in $\mathrm{BCR}$-induced $\mathrm{Ca}^{2+}$ mobilisation of cin $85^{\text {sh }}$ DT40 B cells. (legend on next page) 
Figure 3.7: Ectopic re-expression of cin85 reverses the defect in $\mathrm{BCR}$-induced $\mathrm{Ca}^{2+}$ mobilisation of cin85 $5^{\text {sh }}$ DT40 B cells

A) Wild-type (lanes 1 and 2) or CD2AP-deficient (lanes 3 and 4) DT40 B cells expressing either shRNA against cin85 mRNA (cin85 ${ }^{\text {sh }}$, lanes 2 and 4) or control shRNA (mock ${ }^{\text {sh }}$, lane 1 and 3 ) were transduced with constructs coding for Citrine-tagged CIN85 and cleared cellular lysates were subjected to SDS-PAGE and analysed by immunoblotting with antibodies recognising CIN85 (short or long exposure), chicken CD2AP or actin (upper two, middle and lower panel, respectively). B) Representative histogram of flow cytometric analysis of cells described in A). Gates reflect the Citrinepositive (P2) or Citrine-negative (P1) populations of cells that were analysed in subsequent $\mathrm{Ca}^{2+}$ mobilisation assays. C) and D) Flow cytometric analysis of $\mathrm{BCR}$-induced $\mathrm{Ca}^{2+}$ mobilisation of cells described in A) after gating for Citrine-CIN85 positive (light blue and yellow lines) or negative cells (dark blue and red lines) in either wild-type (C, wt) or cd2ap ${ }^{-/}$DT40 B cells (D, cd2ap ${ }^{\text {neg }}$ ). Apparent molecular weights of protein standards are indicated on the left in $\mathrm{kDa}$.

The cells were analysed for their properties in $\mathrm{BCR}$-induced $\mathrm{Ca}^{2+}$ mobilisation discriminating Citrine-CIN85-negative (P1) or -positive cells (P2) as shown in figure 3.7B. Since the effects of CIN85 were best observable using a low concentration of stimulating antibodies, experiments were only performed with $0.02 \mu \mathrm{g} / \mathrm{ml}$ anti-chicken $\operatorname{lgM}$, hereafter. In accordance with my previous results (figures 3.2 and $3.5 \mathrm{C}$ ), interference with cin85 expression resulted in a delayed and decreased $\mathrm{Ca}^{2+}$ response upon $\mathrm{BCR}$ cross-linking in $\mathrm{wt} / \mathrm{cin} 85^{\mathrm{sh}}$ or cd2ap ${ }^{\text {neg }} / \mathrm{cin}^{\mathrm{sh}} 5^{\text {sh }}$ DT40 B cells, although the defect is more pronounced on cd2ap ${ }^{\%}$ genetic background (figure $3.7 \mathrm{C}$ and $\mathrm{D}$, compare dark blue and red lines). Importantly, when cin85 expression was reintroduced into $w t / c i n 85^{\text {sh }}+$ CIN85 or cd2ap ${ }^{\text {neg }} / \operatorname{cin} 85^{\text {sh }}+$ CIN85 DT40 B cells, the defect in the $\mathrm{Ca}^{2+}$ response could be partially reverted (figures 3.7C or D yellow line).

The magnitude of the BCR-triggered $\mathrm{Ca}^{2+}$ signal did depend on the strength of Citrine-CIN85

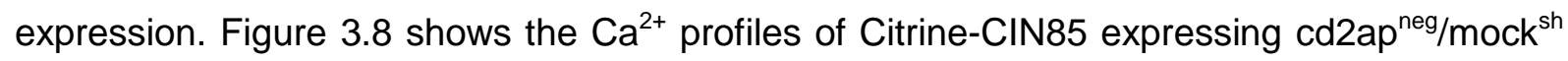
or cd2ap ${ }^{\text {neg }} / \mathrm{cin}^{\mathrm{sh}} 5^{\text {sh }}$ DT40 B cells after gating for negative (P1), medium (P2) and high Citrine expression (P3) (figure 3.8A). In the presence of endogenous CIN85, high overexpression of cin85 resulted in a diminished $\mathrm{Ca}^{2+}$ response compared to Citrine-CIN85 negative cd2ap $^{\text {neg }} /$ mock $^{\text {sh }}$ DT40 B cells (figure $3.8 \mathrm{~B}$, compare green and dark blue line). After interference with endogenous cin85 expression ectopic expression of cin85 resulted in an increase in BCR-induced $\mathrm{Ca}^{2+}$ mobilisation, but the higher the expression of cin85 the smaller was the positive effect on $\mathrm{Ca}^{2+}$ fluxing in cd2ap ${ }^{\text {neg }} / \mathrm{cin}^{2} 5^{\text {sh }}$ DT40 B cells (figure $3.8 \mathrm{C}$ ). In conclusion the re-expression of cin85 reverses the anti-cin85 shRNA effects in the initiation of BCR-induced $\mathrm{Ca}^{2+}$ mobilisation confirming the specific function of CIN85 in this signalling step. However, for optimal BCR-induced $\mathrm{Ca}^{2+}$ mobilisation cin85 expression must be in tightly defined limits. 
A)

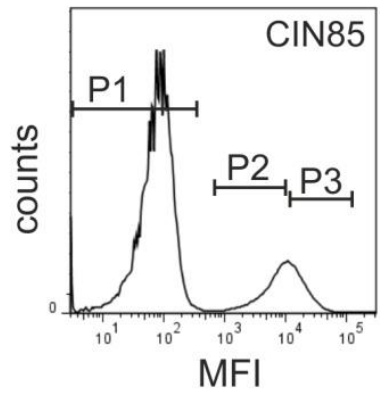

B)

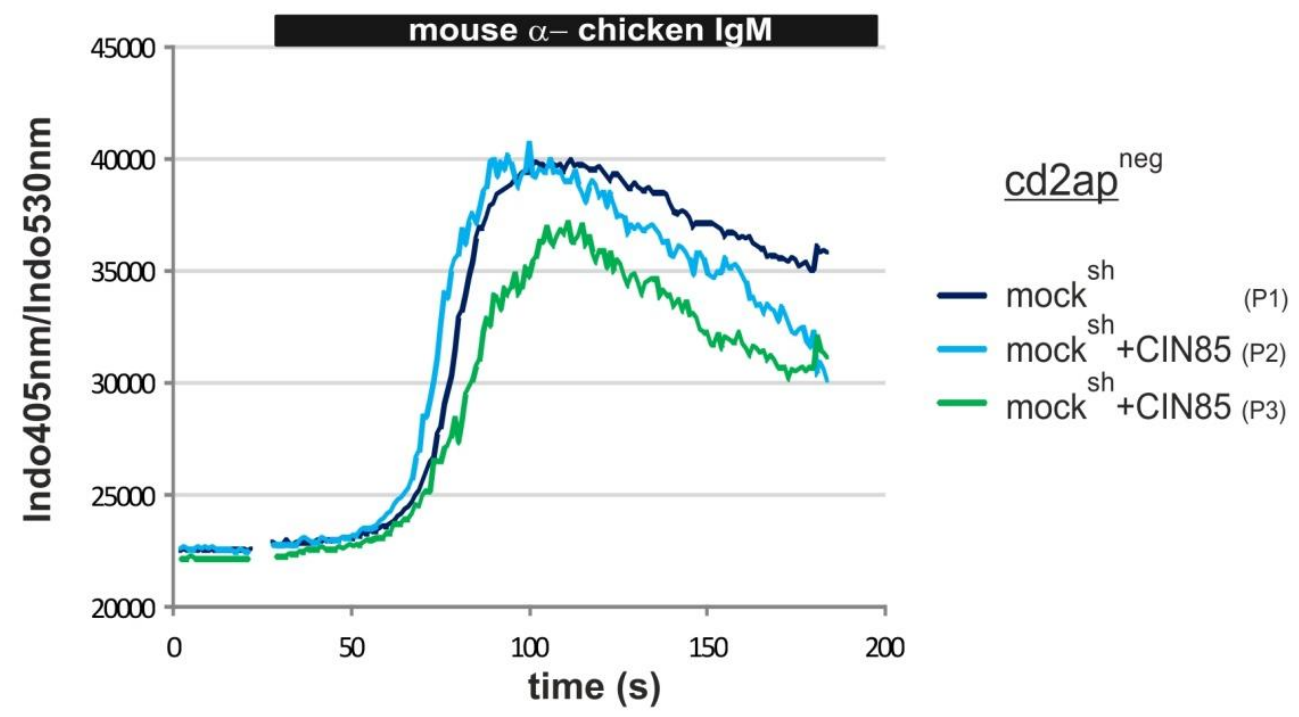

C)

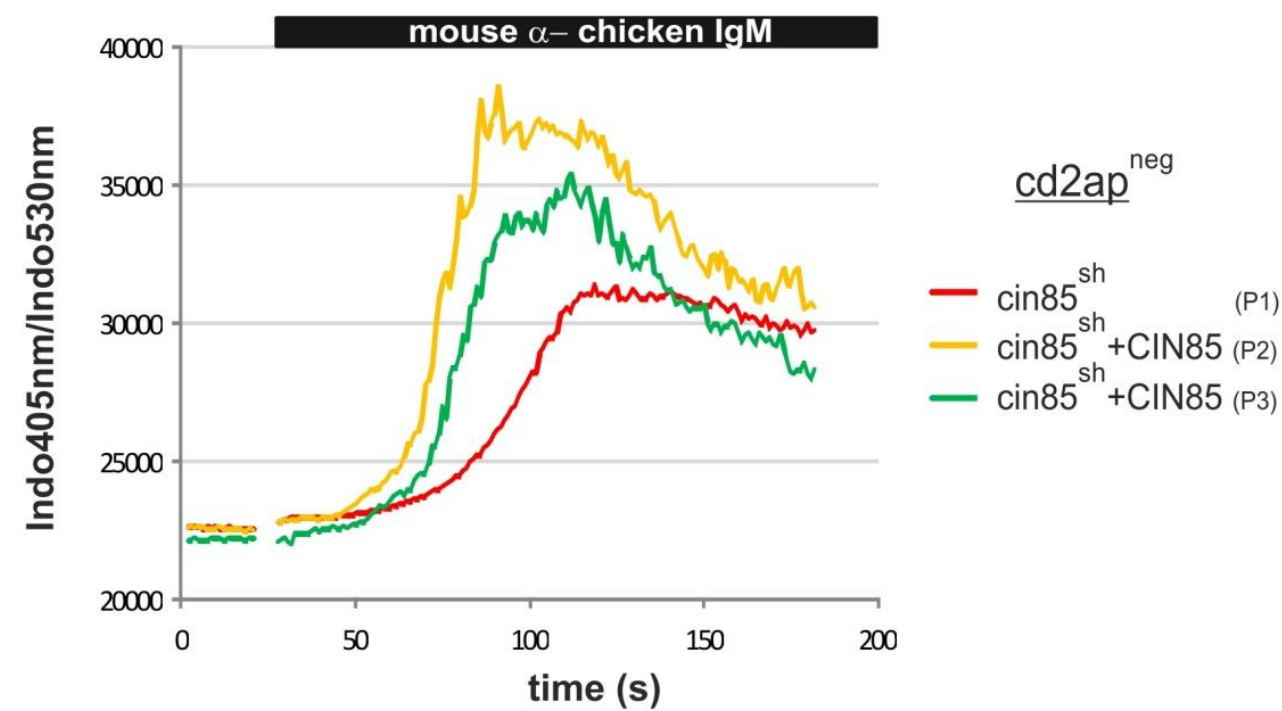

Figure 3.8: The strength of ectopic cin85 expression influences the $\mathrm{BCR}$-induced $\mathrm{Ca}^{2+}$ profile.

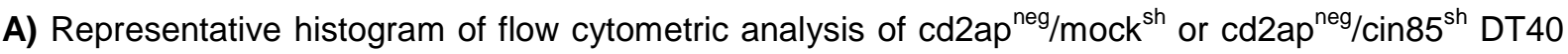
B cells (see figure 3.7) gated for no (P1), medium (P2) or high Citrine expression (P3). B and C) Flow

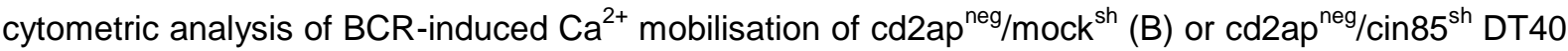
$B$ cells $(C)$ after gating for Citrine-expression as described in $A$ ). 


\subsubsection{CD2AP can rescue the $\mathrm{BCR}$-triggered $\mathrm{Ca}^{2+}$ mobilisation defect induced by diminished cin85 expression}

To investigate whether CD2AP can restore the $\mathrm{Ca}^{2+}$ flux in $\operatorname{cin} 85^{\text {sh }}$ cells, wt $/ \mathrm{mock}^{\text {sh }}$, $\mathrm{wt} / \mathrm{cin} 5^{\text {sh }}, \mathrm{cd} 2 \mathrm{ap}^{\text {neg }} / \mathrm{mock}^{\text {sh }}$ or $\mathrm{cd} 2 \mathrm{ap}{ }^{\text {neg }} / \mathrm{cin} 5^{\text {sh }}$ DT40 B cells were retrovirally transduced to express CD2AP fused to a Citrine-tag (see figure 3.6). Expression of Citrine-tagged CD2AP in the indicated cell types was confirmed by immunostaining with anti-CD2AP antibodies (figure 3.9A, upper panel) and reduction of CIN85 on the protein level was determined using anti-CIN85 antibodies (figure $3.9 \mathrm{~A}, 2^{\text {nd }}$ panel, lanes 2 and 4 ). Cells were selected with puromycin prior to immunoblotting, since the expression of CD2AP was not detectable in the mixed Citrine-CD2AP positive and negative population. Unselected $\mathrm{wt} / \mathrm{mock}^{\mathrm{sh}}$, wt $/ \mathrm{cin}^{\mathrm{s}} 5^{\mathrm{sh}}$, cd2ap $^{\text {neg }} /$ mock $^{\text {sh }}$ or cd2ap $^{\text {neg }} /$ cin85 $5^{\text {sh }}$ DT40 B cells either Citrine-CD2AP positive (P2) or negative (P1) were subjected to $\mathrm{BCR}$-induced $\mathrm{Ca}^{2+}$ flux analysis after gating on the respective populations as shown in figure 3.9B.

Overexpression of cd2ap on a wild-type genetic background did not have an impact on the BCR-induced $\mathrm{Ca}^{2+}$ signal, but in $\mathrm{wt} / \mathrm{cin}^{2} 5^{\text {sh }}$ DT40 B cells cd2ap expression shifted the moderately reduced and delayed $\mathrm{Ca}^{2+}$ signal towards the $\mathrm{Ca}^{2+}$ profile of mock $^{\text {sh }}$ cells (figure $3.9 \mathrm{C}$, compare red line with yellow line). The described defect was more pronounced in cd2ap ${ }^{\text {neg }} / \mathrm{cin}^{\mathrm{sh}}$ DT40 B cells (figure 3.9D) and could be rescued upon re-expression of cd2ap in these cells $\left(\mathrm{cd} 2 a p^{\text {neg }} / \mathrm{cin} 5^{\mathrm{sh}}+\mathrm{CD} 2 \mathrm{AP}\right.$, yellow line). Note that the genetic reconstitution experiment of $\mathrm{cd}_{2} \mathrm{ap} \mathrm{p}^{\text {neg }} / \mathrm{mock}^{\text {sh }}$ with $\mathrm{CD} 2 \mathrm{AP}$ is similar to the reconstitution experiment of cd2ap \% DT40 B cells with CD2AP (see figure 3.4) and both experiments showed no difference in BCR-induced $\mathrm{Ca}^{2+}$ mobilisation between the CD2AP-deficient and the reconstituted DT40 B cells.

A)

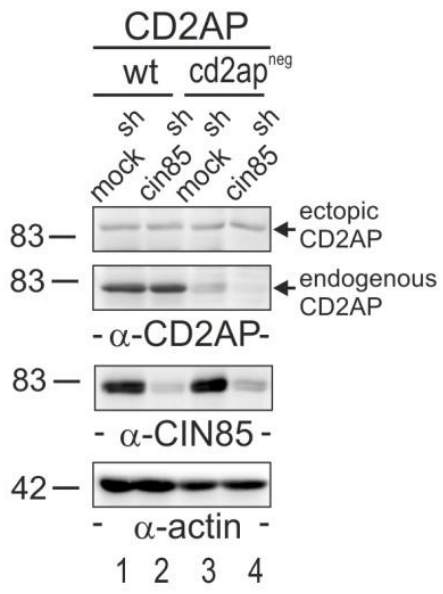

B)

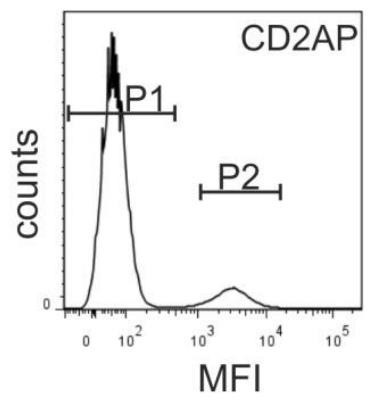

(figure continues on next page) 
C)

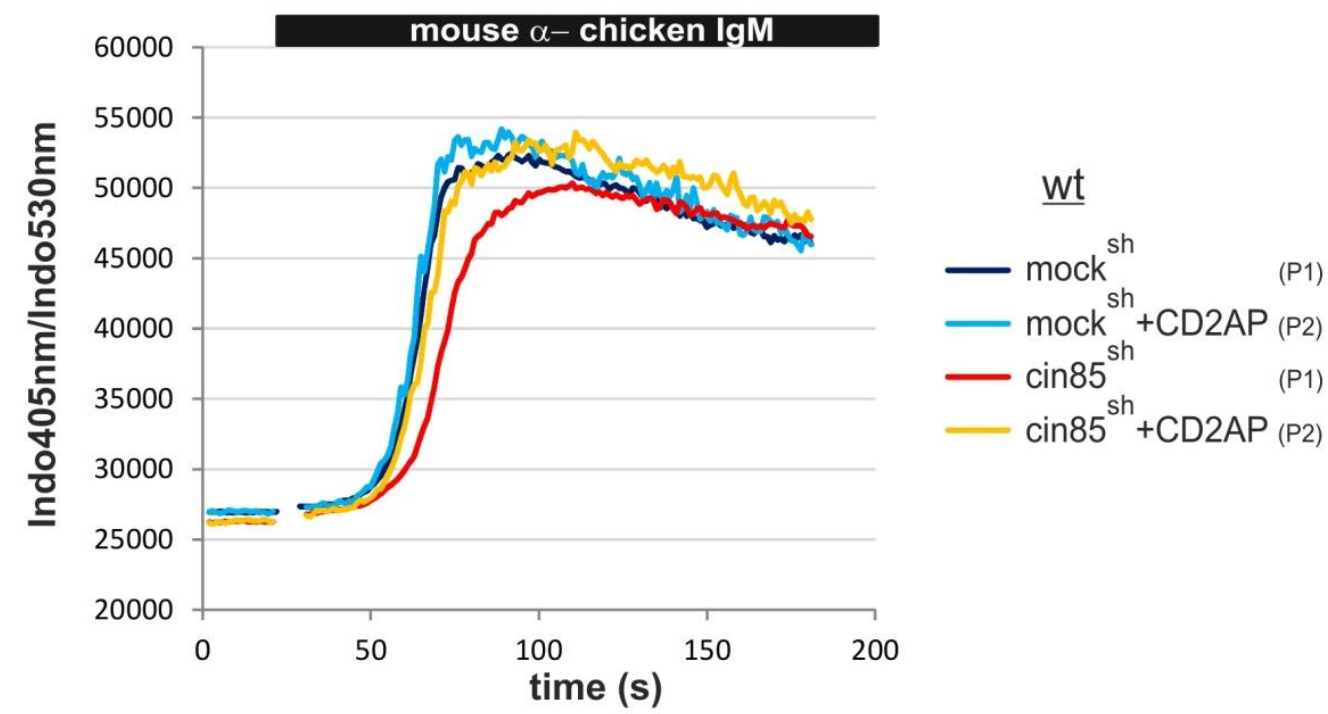

D)

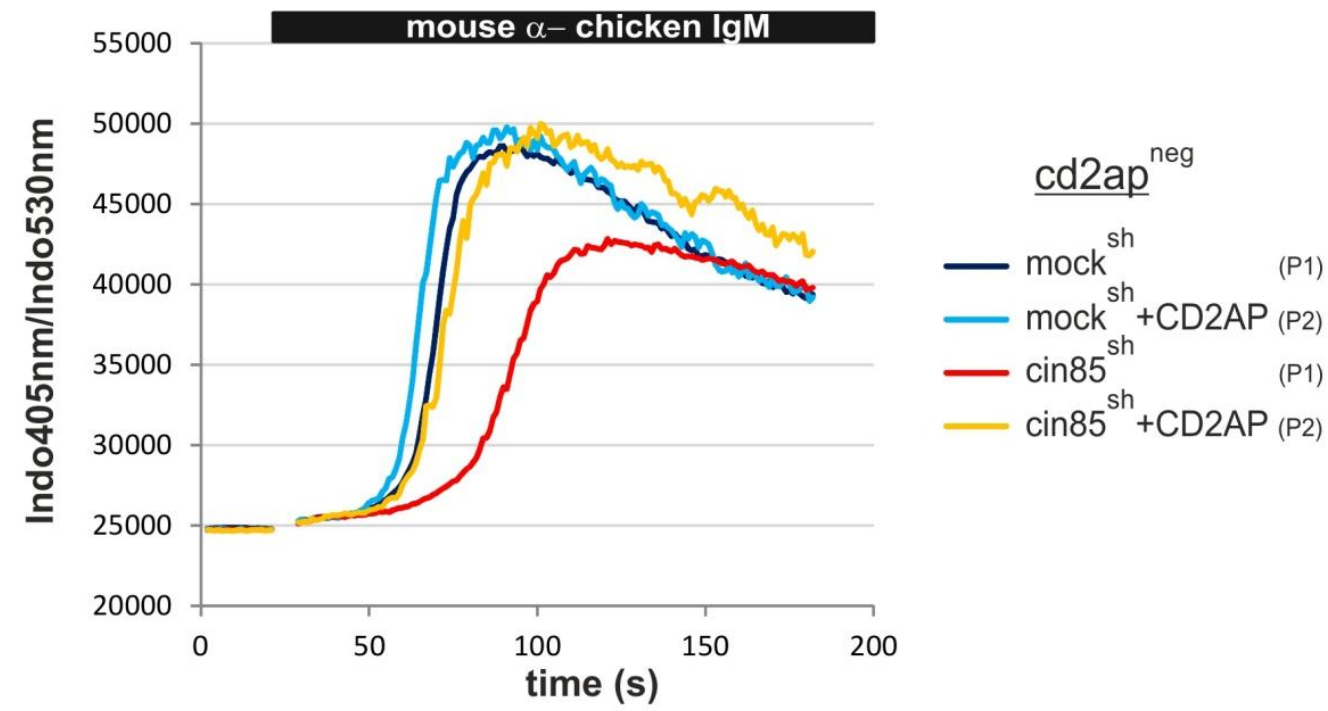

Figure 3.9: $\mathrm{CD2AP}$ can rescue the $\mathrm{Ca}^{2+}$ mobilisation defect induced by diminished cin85 expression.

A) Wild-type or cd2ap \% DT40 B cells were retrovirally transduced to express an shRNA against cin85 mRNA (cin85 $5^{\text {sh }}$, lanes 2 and 4) or a control shRNA (mock ${ }^{\text {sh }}$, lanes 1 and 3) prior to transfection with constructs coding for Citrine-tagged CD2AP. After puromycin selection of the cells, cleared cellular lysates were subjected to SDS-PAGE followed by immunoblotting with antibodies against human CD2AP, CIN85, chicken CD2AP or actin (from top to bottom). B) Representative histogram obtained by flow cytometry of cells described in A). Gates reflect the Citrine-negative (P1) or Citrine-positive (P2) populations separately analysed in C) and D). C) and D) Flow cytometric analysis of BCRinduced $\mathrm{Ca}^{2+}$ mobilisation of cells described in A) after gating for Citrine-CD2AP positive (light blue and yellow lines) or negative cells (dark blue and red lines) in either wild-type (C, wt) or cd2ap ${ }^{-/} \mathrm{DT} 40$ $B$ cells $\left(D, c d 2 a p^{\text {neg }}\right)$. Apparent molecular weights of protein standards are indicated on the left in kDa. 
In conclusion, CIN85 regulated the onset of the $\mathrm{Ca}^{2+}$ signal of BCR-activated DT40 B cells in a positive manner and CD2AP was partially redundant in exerting this function. Upon crosslinking of the BCR, DT40 B cells deficient for CD2AP and diminished in cin85 expression displayed a kinetic and magnitudinal defect in $\mathrm{Ca}^{2+}$ mobilisation that could be reverted by ectopical expression of either of the two proteins. This impaired $\mathrm{Ca}^{2+}$ profile is similar to the $\mathrm{Ca}^{2+}$ defect observed for the SLP65 variant in which binding to CIN85/CD2AP was abolished.

\subsection{The preformed complex of SLP65 and CIN85/CD2AP is based on the interaction of the SH3 domains of CIN85/CD2AP and atypical proline- arginine motifs in SLP65}

\subsubsection{The SH3 domains of CIN85 and CD2AP bind to atypical proline-arginine motifs in SLP65}

The biochemical prerequisites for the formation of the preformed complex comprising CIN85/CD2AP and SLP65 were investigated next. Figure 3.10A shows the location and amino acid sequence of the three atypical proline-arginine motifs in SLP65. These motifs can be destroyed by an exchange of the arginine $(R)$ with an alanine $(A)$ residue in the last position eliminating CIN85 and CD2AP binding (Kowanetz et al. 2003).

To test if the SH3 domains of CIN85 and CD2AP recognise the proline-arginine motifs in SLP65 in the DT40 system affinity purifications with Glutathione S-transferase (GST) fusion proteins were performed (figure 3.10). s/p65 \% DT40 B cells were transfected with constructs encoding GFP-tagged wild-type SLP65 (wt) or a SLP65 variant in which all three binding sites for CIN85/CD2AP were removed (M1-3) (cells kindly provided by T.Oellerich). Equal expression of proteins was confirmed by immunostaining with SLP65 antibodies (figure 3.10B and D, left panel). Cleared cellular lysates were incubated with a GST fusion protein comprising either the three $\mathrm{SH} 3$ domains of CIN85 or CD2AP (figure 3.10B and D, right panel, respectively). The SH3 domains of CIN85 as well as those of CD2AP precipitated wild-type, but not the R-to-A variant of SLP65, independent of BCR activation (figure 3.10B and $D$, right panel, lane 3 and 4 or lanes 5 and 6 ). Cleared cellular lysates were also incubated with GST alone as a negative control (data not shown).

Figure $3.10 \mathrm{C}$ shows an in vitro experiment in which only the two fusion proteins GSTCIN85(SH3) ${ }_{3}$ and $\mathrm{His}_{6}$-SLP65 (lane 2) were mixed and the GST protein was precipitated. It revealed a direct interaction of the SH3 domains of CIN85 with SLP65 in vitro. 
A)

hSLP65

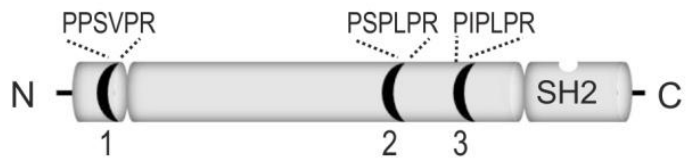

B)

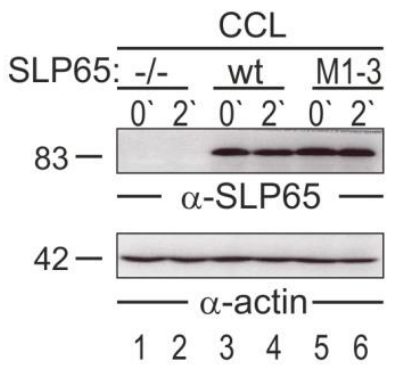

D)

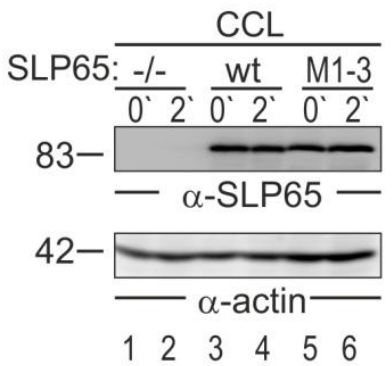

GST-

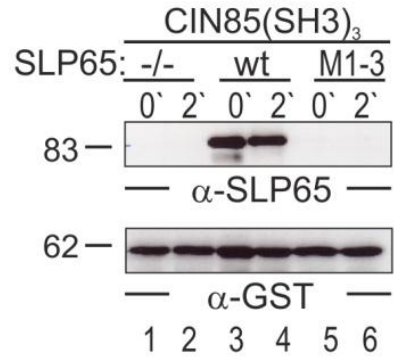

C)

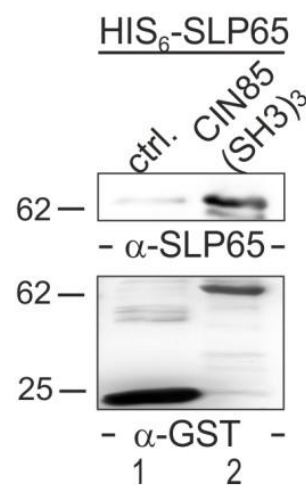

Figure 3.10: The SH3 domains of CIN85 and CD2AP bind to atypical proline-arginine motifs in SLP65.

A) Schematic drawing of human SLP65 with the three atypical proline-arginine motifs $(1,2$, and 3 , black). Respective primary structures of the motifs are written in the single letter amino acid code. B) and D) SLP65-deficient (-/-, lane 1 and 2) or s/p65 DT40 B cells reconstituted with GFP-tagged forms of either human wild-type SLP65 (wt, lane 3 and 4) or a mutant version harbouring R-to-A amino acid substitutions in all three atypical proline-arginine motifs (M1-3, lane 5 and 6) were left untreated $\left(0^{\prime}\right)$ or BCR-stimulated for 2 min (2'). Cleared cellular lysates were analysed by immunoblotting (CCL, left panel) or were incubated with GST-fusion proteins encompassing the three SH3 domains of human CIN85 (B) or CD2AP (D), subjected to SDS-PAGE and affinity-purified proteins were analysed by immunoblotting with antibodies recognising SLP65 or GST (right panel, upper and lower blot, respectively). C) The GST-CIN85(SH3) ${ }_{3}$ protein described in A) or GST alone as a negative control were incubated with recombinantly expressed $\mathrm{His}_{6}$-SLP65 and immobilised on glutathione sepharose beads. Affinity-purified proteins were separated with SDS-PAGE followed by immunoblot analysis with anti-SLP65 (upper panel) or anti-GST antibodies (lower panel). Apparent molecular weights of protein standards are indicated on the left in $\mathrm{kDa}$. Parts of this figure are published in (Oellerich et al. 2011) 


\subsubsection{CIN85 and CD2AP bind the second and third atypical proline-arginine motif in SLP65}

Next, the contribution of each proline-arginine motif in SLP65 to the interaction with CIN85/CD2AP was explored. Therefore, DT40 s/p65 $\%$ B cells were transfected with constructs encoding wild-type or mutants of s/p65. The introduced mutations resulted in R-toA substitutions at positions 49 (M1), 248 (M2), 313 (M3), 248,313 (M23) or 49,248,313 (M1 3) of SLP65 (compare figure 3.10A).

Cleared cellular lysates of the indicated DT40 B cells were subjected to anti-GFP immunoprecipitation and purified proteins were analysed by immunoblotting (figure 3.11, cells kindly provided by T.Oellerich). Wild-type SLP65 was purified with CIN85 as well as CD2AP independent of BCR stimulation (figure 3.11, lanes 1 and 2). SLP65_M1 bound the same or higher (increased binding was not observed in all experiments performed) amounts of CIN85 or CD2AP as did SLP65_wt (lanes 3 and 4), in contrast to SLP65_M2 in which the binding to CIN85 and CD2AP was abolished (lanes 5 and 6). An R-to-A amino acid exchange in the third motif (SLP65_M3) resulted in a weakened interaction with CIN85 and CD2AP compared to SLP65_wt purifications (lanes 7 and 8). As soon as the second prolinearginine motif was altered, CIN85 and CD2AP did not purify with SLP65 any more (SLP65_M23, lanes 9 and 10, SLP65_M1-3, lanes 11 and 12).

Thus, the SH3 domains of CIN85 and those of CD2AP recognise mainly the second prolinearginine motif in SLP65 though the third motif contributes to the interaction. Thus, both CIN85 and CD2AP had the same preferred SLP65 binding site and did not differ in their mode of binding to SLP65.

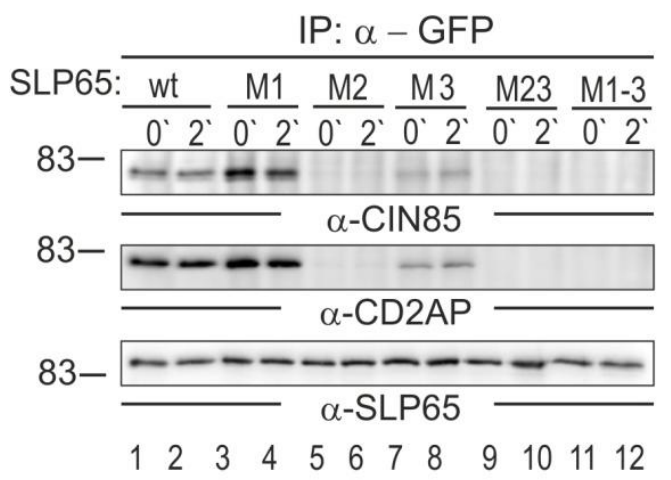

Figure 3.11: CIN85 and CD2AP preferentially bind the second atypical proline-arginine motif in SLP65.

SLP65-deficient DT40 B cells expressing GFPtagged human wild-type (lanes 1 and 2) or mutants of SLP65 containing one (lanes 3-8), two (lanes 9 and 10) or three (lanes 11-12) Rto-A amino acid exchanges, were left untreated $\left(0^{\prime}\right)$ or stimulated via the BCR (2') and subjected to anti-GFP immunoprecipitation. The proteins were separated by SDS-PAGE and analysed by anti-CIN85, anti-chicken CD2AP or anti-SLP65 immunoblotting (upper, middle and lower panel, respectively). Apparent molecular weights of protein standards are indicated on the left in $\mathrm{kDa}$. 


\subsubsection{CIN85 and CD2AP can hetero-oligomerise via coiled coil domains}

To gain further insight into the stoichiometry of the CIN85/CD2AP and SLP65 complex, I tested for the possibility of higher order complexes due to hetero-oligomerisation of CIN85 and CD2AP via their coiled coil domains. N-terminally Citrine-tagged CIN85 or the coiled coil truncated variant were expressed in DT40 wild-type B cells and purified from lysates of unstimulated or BCR-stimulated cells with anti-GFP antibodies. Immunoblot analysis with anti-CD2AP antibodies revealed that full length, but not the coiled coil truncation variant of CIN85 purified CD2AP (figure 3.12, lanes 1-3 and lanes 4-6, respectively).

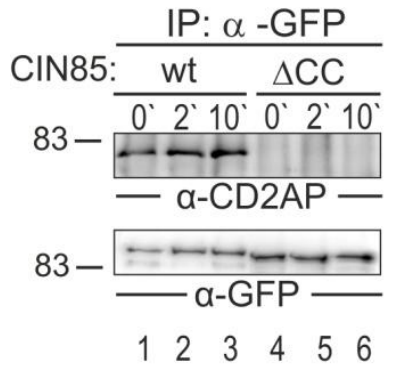

\begin{abstract}
Figure 3.12: CIN85 and CD2AP can hetero-oligomerise via coiled coil domains.

Anti-GFP immunoprecipitates derived from unstimulated $\left(0^{\circ}\right)$ or BCR-stimulated (2') wild-type DT40 B cells expressing either Citrine-tagged full-length human CIN85 (lanes 1-3) or a coiled coil truncation variant (lanes 4-6) were analysed by SDS-PAGE followed by anti-chicken CD2AP or anti-GFP immunoblotting (upper and lower panel, respectively). Apparent molecular weights of protein standards are indicated on the left in $\mathrm{kDa}$.
\end{abstract}

\subsubsection{CIN85 and CD2AP bind to SLP65 independent of each other}

The interaction of CIN85/CD2AP with SLP65 could be either direct or indirect when binding of CD2AP is mediated via CIN85 or vice versa. To check for dependence, I used the established $c d 2 a p \%$ DT40 B cells or the RNAi approach (figure 3.13).

Figure 3.13A shows anti-GFP immuno purifications of either SLP65_wt (lanes 1-4) or SLP65_M23 (lanes 5-8) from lysates of wild-type (wt) or cd2ap ${ }^{-/-}(-/-)$DT40 B cells. The SLP65_M23 variant did neither bind CIN85 nor CD2AP and thus served as a negative control in this experiment (see figure 3.11). Wild-type SLP65 bound CIN85 and CD2AP (figure 3.13, lanes 1 and 2) and importantly, the association of SLP65_wt and CIN85 was not affected by the absence of CD2AP (lanes 3 and 4). This is underscored by the ability of recombinant SLP65 to interact directly with the SH3 domains of CIN85 (see figure 3.10C). The reverse experiment was performed with mock $^{\text {sh }}$ or cin85 8 sh DT40 B cells expressing Citrine-tagged wild-type SLP65. Anti-GFP immuno purifications of SLP65 revealed an association of SLP65 with CD2AP in mock ${ }^{\text {sh }}$ cells as well as in cin85 $5^{\text {sh }}$ DT40 B cells, although the interaction of CIN85 with SLP65 is considerably reduced in the latter cells (lanes 5 and 6). Thus, CD2AP and CIN85 could bind to SLP65 independent of each other. 
A)

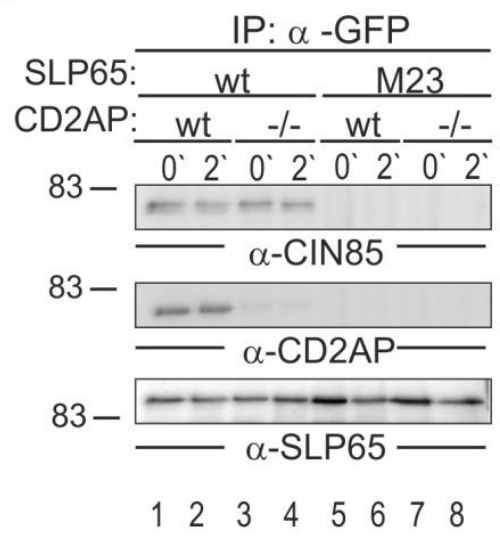

B)

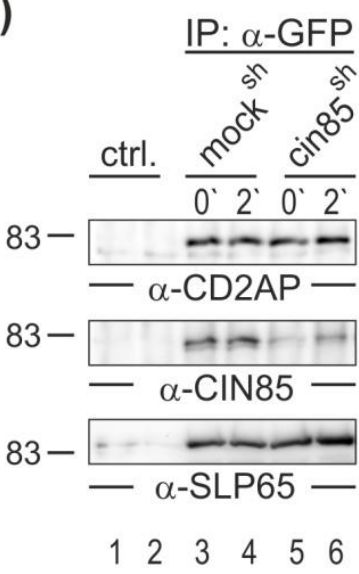

Figure 3.13: CIN85 and CD2AP bind to SLP65 independent of each other.

A) Wild-type (lanes 1, 2, 5 and 6) or CD2AP-deficient (lanes 3, 4, 7 and 8) DT40 B cells expressing Citrine-tagged forms of either human wild-type SLP65 (lanes 1- 4) or the SLP65_M23 variant (lanes 5-

8) or B) DT40 wild-type B cells retrovirally transduced to express shRNA against cin85 mRNA (cin85 $5^{\text {sh }}$, lanes 2, 5 and 6) or control shRNA ( mock $^{\text {sh }}$, lanes 1,3 and 4) and Citrine-tagged human wildtype SLP65 (lanes 1-6) were left untreated (0') or BCR-stimulated (2') and subjected to anti-GFP immunoprecipitation or isotype-matched control antibodies (ctrl., B) lanes 1 and 2). After SDS-PAGE the purified proteins were analysed by immunoblotting with antibodies to CIN85 (A) upper, B) middle panel), chicken CD2AP (A) middle B) upper panel) or SLP65 (lower panel). Apparent molecular weights of protein standards are indicated on the left in $\mathrm{kDa}$.

In summary, the presented biochemical data showed that the interaction of CIN85/CD2AP with SLP65 existed prior to BCR stimulation, thus these proteins were preformed. Moreover, the mode of binding of CIN85 as well as CD2AP with SLP65 was identical by biochemical means in that both proteins preferred binding to the second proline-arginine motif in SLP65, but needed the third motif as well. Lastly, CIN85 and CD2AP bound to SLP65 independent of each other and could mediate higher order protein complexes by oligomerisation via their coiled coil domains.

\subsection{CIN85 and CD2AP are recruited to the plasma membrane in BCR-activated B lymphocytes using different anchoring modes}

The BCR-triggered events leading to $\mathrm{Ca}^{2+}$ release are characterised by plasma membrane translocation of the proteins involved. To test whether CIN85 and CD2AP also obey to this principle, I investigated the location of CIN85 and CD2AP and changes in their localisation upon BCR activation in DT40 B cells by live cell confocal microscopy (figure 3.14). 
A)

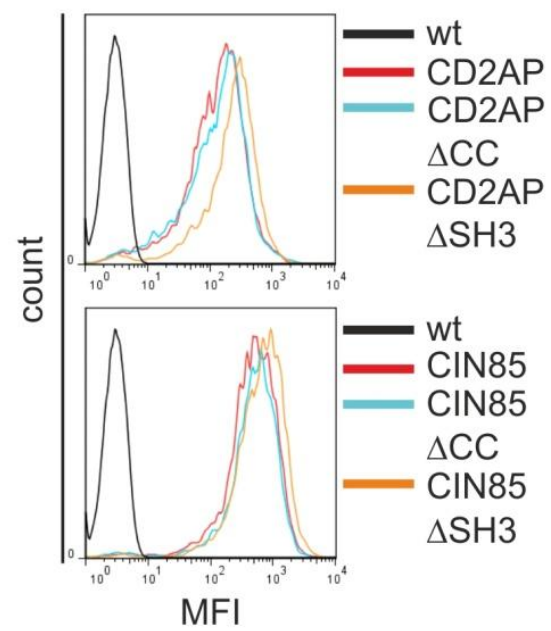

B)
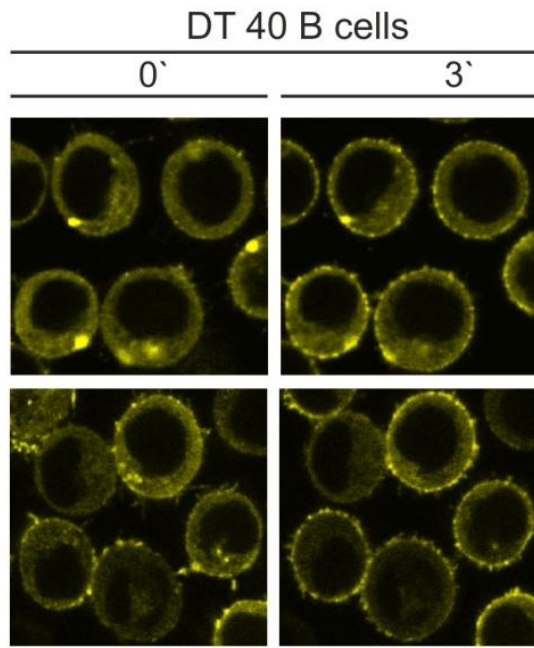

D)

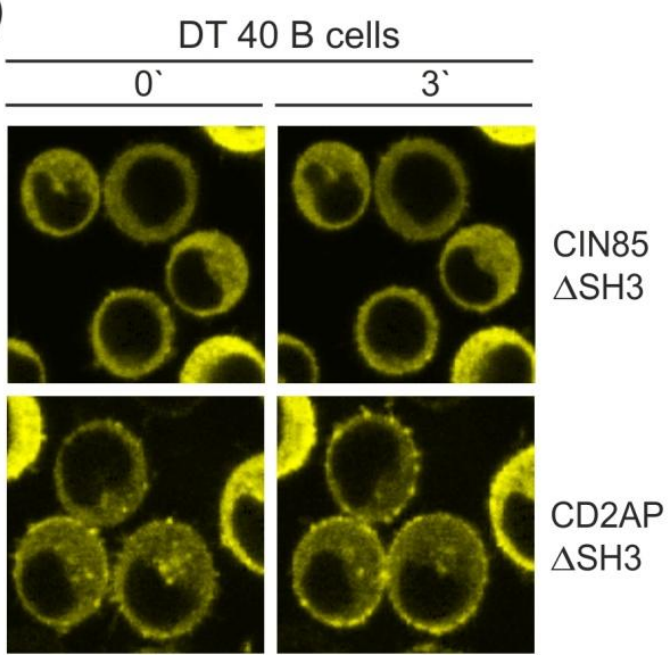

Figure 3.14: CIN85 and CD2AP translocate to the plasma membrane after stimulation of the BCR, but use different anchoring modes.

A) Wild-type DT40 B cells were retrovirally transduced to express either $\mathrm{N}$-terminally Citrine-tagged human CD2AP or human CIN85 (CD2AP or CIN85) or truncated variants which either lack all three $\mathrm{SH} 3$ domains (CD2AP $\triangle \mathrm{SH} 3$ or $\mathrm{CIN} 85 \Delta \mathrm{SH} 3$ ) or the coiled coil domain (CD2AP $\triangle \mathrm{CC}$ or CIN85 $\triangle \mathrm{CC}$ ). Cells were analysed for Citrine expression by flow cytometry (blue, red and orange lines). Untransfected wild-type DT40 cells (wt) served as negative control (black line). B-E) Confocal laser scanning microscopy of cells expressing full-length (B and C) or SH3 (D) or coiled coil (E) truncated variants of CIN85 or CD2AP (upper and lower panel, respectively) pre-treated with $2 \mu \mathrm{M}$ Latrunculin $\mathrm{B}$ for $15 \mathrm{~min}$ (C) prior to or $3 \mathrm{~min}$ after BCR-stimulation (left and right panels, respectively). Parts of this figure are published in (Oellerich et al. 2011)

C)

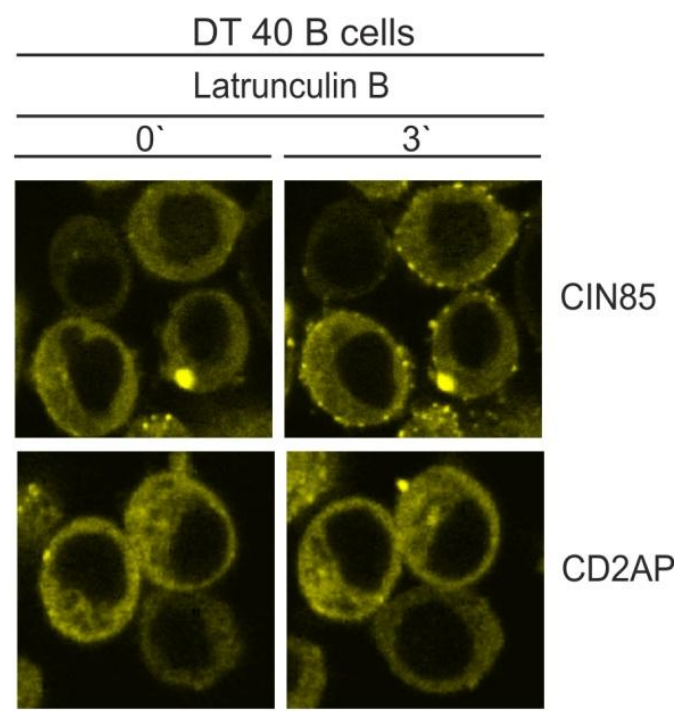

E)

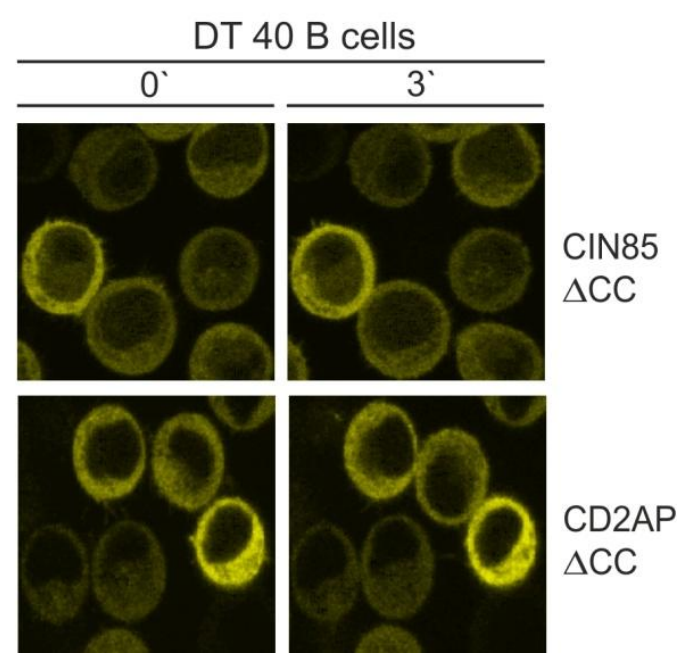


Wild-type DT40 B cells were transfected with constructs coding either for Citrine-tagged CIN85 or Citrine-tagged CD2AP or their respective truncation variants and the expression of the Citrine fusion proteins was confirmed by flow cytometry (figure 3.14.A). Table 3.1 shows the quantitative analysis of Citrine-CIN85 and Citrine-CD2AP fluorescence at the plasma membrane in resting ( $\left.M_{\text {rest. }}\right)$ and BCR-stimulated ( $\left.M_{\text {stim. }}\right)$ DT40 B cells (for details see 2.2.4).

Figure 3.14B shows that in resting cells CIN85 resided mainly in the cytoplasm, which equals an $\mathrm{M}_{\text {rest }}(\mathrm{CIN85})=1.07$, meaning there is comparable fluorescence at the plasma membrane and in the cytosol (figure 3.14B, upper panel, left image). After BCR activation, CIN85 translocated towards the plasma membrane, where it built a dotted, ring like structure (figure 3.14B, upper panel, right image). Thus, in BCR-stimulated cells more CIN85 was found at the plasma membrane, which is reflected by an $M_{\text {stim. }}(C I N 85)>1=1.36$. CD2AP was mainly cytoplasmatically localised, but could also be detected in vesicular structures and at membrane ruffles (figure 3.14B, lower panel, left image). Increased amounts of CD2AP were found at the plasma membrane after BCR stimulation (figure $3.14 \mathrm{~B}$, lower panel, right image).

Next, I investigated the mechanism of membrane recruitment of CD2AP and CIN85. There is recent evidence that the membrane skeleton controls BCR signalling by restriction of free BCR diffusion within the plasma membrane of B cells (Treanor et al. 2009). Since CIN85 and CD2AP are referred to as cytoskeletal adaptor proteins, I analysed their membrane localisation with respect to an integral cytoskeleton.

Therefore, the Citrine-tagged CIN85 or Citrine-tagged CD2AP expressing DT40 B cells were treated with the toxin Latrunculin $B$, which disrupts microfilament organisation and microfilament-mediated processes (Spector et al. 1989) (figure 3.14C). After BCR activation of Latrunculin B-treated B cells, CIN85 could be recruited to the plasma membrane, although the amount and pattern of CIN85 at the membrane was different compared to untreated cells (compare figure 3.14B and $C$, upper panel or $M_{\text {stim. }}$ (CIN85) =1.36 and $M_{\text {stim. }}$ (CIN85 Latrunculin $B)=1.21$ ). CD2AP was neither found at membrane ruffles prior to nor at the membrane after BCR stimulation in Latrunculin B-treated B cells.

Next, I evaluated the importance of the SH3 and coiled coil domains of CIN85 and CD2AP for membrane translocation. Therefore wild-type DT40 B cells were retrovirally transduced to express Citrine-tagged SH3- or coiled coil domain truncation variants. When not equipped with its SH3 domains, CIN85 was not able to inducibly locate to the plasma membrane (figure 3.14D, upper panel, right image). In contrast, CD2AP $\triangle \mathrm{SH} 3$ could still be recruited to the plasma membrane, though the amount of $\mathrm{CD} 2 \mathrm{AP} \Delta \mathrm{SH} 3$ at the membrane was lower before and after BCR stimulation compared to wild-type CD2AP (see table 3.1 and figure 3.14D, lower panel, right image). Figure 3.14E shows that upon truncation of the coiled coil 
domain, both CD2AP $\triangle \mathrm{CC}$ and $\mathrm{CIN} 85 \Delta \mathrm{CC}$ could not attach to the plasma membrane anymore.

Table 3.1: Quantification of plasma membrane localisation of CIN85 or CD2AP and their respective variants in resting or BCR-activated DT40 B cells

\begin{tabular}{ccccc}
\hline DT40 B cell specimen & $\mathbf{M}_{\text {rest. }}$ & $\mathbf{M}_{\text {stim. }}$ & fold-change & number of cells \\
\hline CIN85 & $\mathbf{1 . 0 7} \pm 0.02$ & $\mathbf{1 . 3 6} \pm 0.12$ & $\mathbf{1 . 2 8} \pm 0.13$ & $\mathrm{n}=75$ \\
CD2AP & $\mathbf{1 . 4 3} \pm 0.18$ & $\mathbf{1 . 8 5} \pm 0.26$ & $\mathbf{1 . 3 3} \pm 0.15$ & $\mathrm{n}=89$ \\
CIN85 Latrunculin B & $\mathbf{1 . 0 2} \pm 0.01$ & $\mathbf{1 . 2 1} \pm 0.01$ & $\mathbf{1 . 2 1} \pm 0.02$ & $\mathrm{n}=41$ \\
CD2AP Latrunculin B & $\mathbf{1 . 0 0} \pm 0.03$ & $\mathbf{1 . 0 1} \pm 0.13$ & $\mathbf{1 . 0 3} \pm 0.09$ & $\mathrm{n}=38$ \\
CIN85 $\Delta S H 3$ & $\mathbf{1 . 0 4} \pm 0.05$ & $\mathbf{1 . 1 1} \pm 0.06$ & $\mathbf{1 . 0 8} \pm 0.03$ & $\mathrm{n}=57$ \\
CD2AP $\Delta S H 3$ & $\mathbf{1 . 1 4} \pm 0.1$ & $\mathbf{1 . 5 6} \pm 0.11$ & $\mathbf{1 . 3 8} \pm 0.03$ & $\mathrm{n}=63$ \\
CIN85 $\Delta C C$ & $\mathbf{0 . 9 6} \pm 0.03$ & $\mathbf{1 . 0 0} \pm 0.01$ & $\mathbf{1 . 0 5} \pm 0.03$ & $\mathrm{n}=45$ \\
CD2AP $\Delta C C$ & $\mathbf{0 . 9 8} \pm 0.09$ & $\mathbf{1 . 0 4} \pm 0.12$ & $\mathbf{1 . 0 7} \pm 0.04$ & $\mathrm{n}=53$
\end{tabular}

Relative membrane localisation (M) of Citrine-tagged CIN85 and CD2AP variants expressed in DT40 $B$ cells without or with prior Latrunculin $B$ treatment. $M$ reflects the mean fluorescence intensity at the plasma membrane normalised to the fluorescence intensity in the cytosol in either unstimulated $\left(\mathrm{M}_{\text {rest. }}\right)$ or 3 min BCR-stimulated DT40 B cells ( $M_{\text {stim. }}$.). The fold-change equals the mean of the ratios of $M_{\text {stim. }}$ and $M_{\text {rest. }}$ calculated for each cell. Quantification ( $\pm S D$ ) of at least triplicates representative for at least two independent experiments is shown (except CD2AP $\triangle C C$, one independent experiment). See section 2.2.4 for details.

In summary, CIN85 and CD2AP translocated to the plasma membrane in response to BCR stimulation. Regarding the underlying mechanisms, CIN85 and CD2AP differed in their need for a functional actin cytoskeleton; it was rather CD2AP that relied on the integrity of this cellular microfilament. Moreover, CD2AP was less sensitive to truncation of its SH3 domains, compared to CIN85 for which the C-terminal half of the protein was not sufficient for plasma membrane localisation. The coiled coil domain was identified as an important mediator for membrane attachment for both CIN85 and CD2AP. 


\subsection{The SH3 and coiled coil domains of CIN85 mediate both BCR-induced membrane recruitment and $\mathrm{Ca}^{2+}$ flux}

\subsubsection{The SH3 and coiled coil domains of CIN85 are indispensable for full $\mathrm{Ca}^{2+}$ signalling in BCR-activated cells}

If the inducible membrane recruitment of CIN85 is a requisite for an appropriate BCRinduced $\mathrm{Ca}^{2+}$ signal, the domains involved in membrane recruitment should also be important for $\mathrm{Ca}^{2+}$ mobilisation. To test for this hypothesis, I used the RNAi system described in figure 3.6 and infected wild-type or cd2ap ${ }^{-1}$ DT40 B cells with mock shRNA or shRNA against cin85 mRNA. Subsequently cells were transduced with constructs coding for cin85 deleted in either the sequences encoding the three $\mathrm{SH} 3(\Delta \mathrm{SH} 3)$ domains or the coiled coil $(\Delta \mathrm{CC})$ domain. Expression of the constructs and reduction in endogenous cin85 expression was confirmed by immunoblotting (figures $3.15 \mathrm{~A}$ or $3.16 \mathrm{~A}$, respectively).

BCR-induced $\mathrm{Ca}^{2+}$ mobilisation was analysed after gating on Citrine-CIN85 $\triangle \mathrm{SH} 3$ or CitrineCIN85 $\triangle \mathrm{CC}$ positive (P2) or negative (P1) DT40 B cells (figures 3.15A or 3.16A, respectively). Expression of cin $85 \Delta s h 3$ could not revert the delay and decrease in BCR-induced $\mathrm{Ca}^{2+}$ mobilisation seen in $w t / c i n 85^{\text {sh }}$ and $c d 2 a p^{\text {neg }} / \mathrm{cin} 85^{\text {sh }}$ DT40 B cells, but rather led to an inhibition of $\mathrm{BCR}$-induced $\mathrm{Ca}^{2+}$ mobilisation (figure 3.15C, compare dark and light blue lines or red and yellow lines).

A)

B)
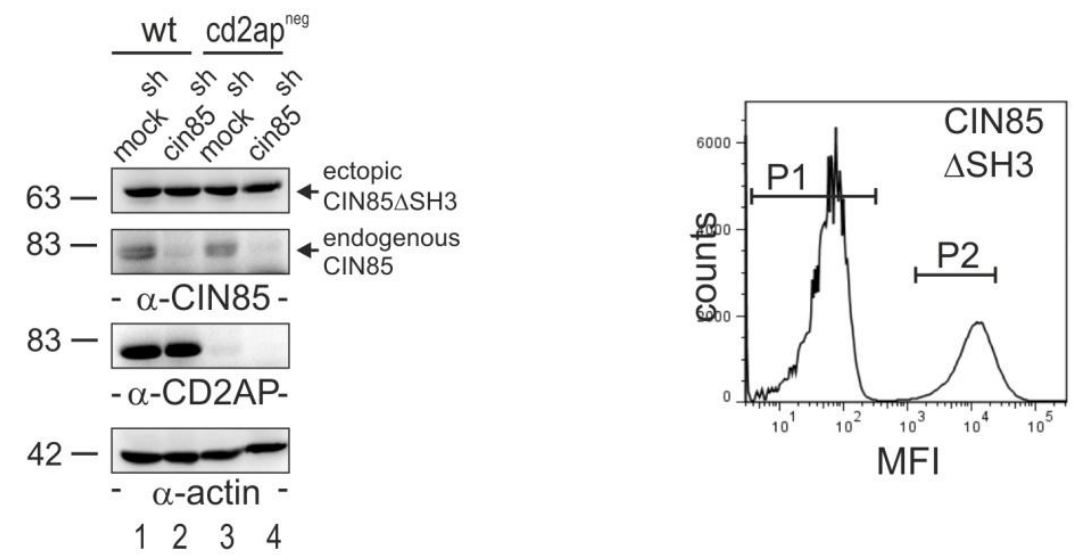

(figure continues on next page) 
C)

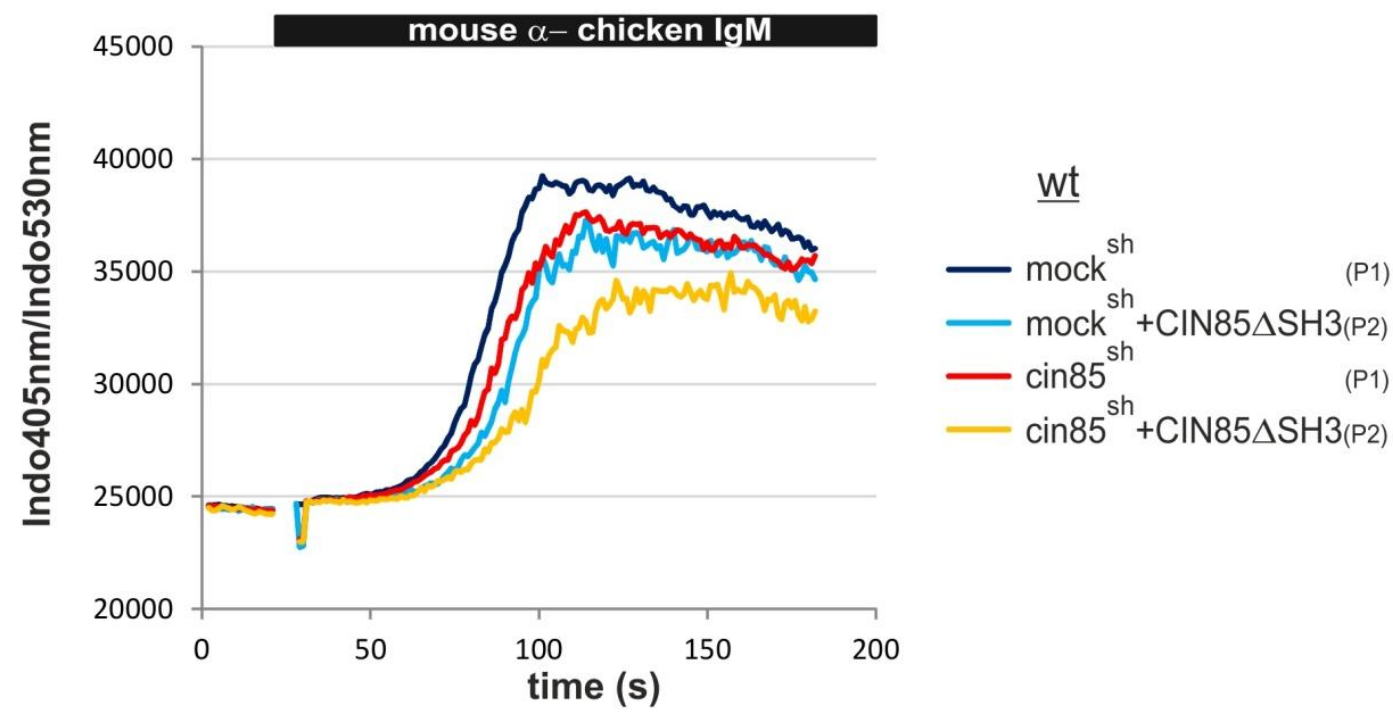

D)

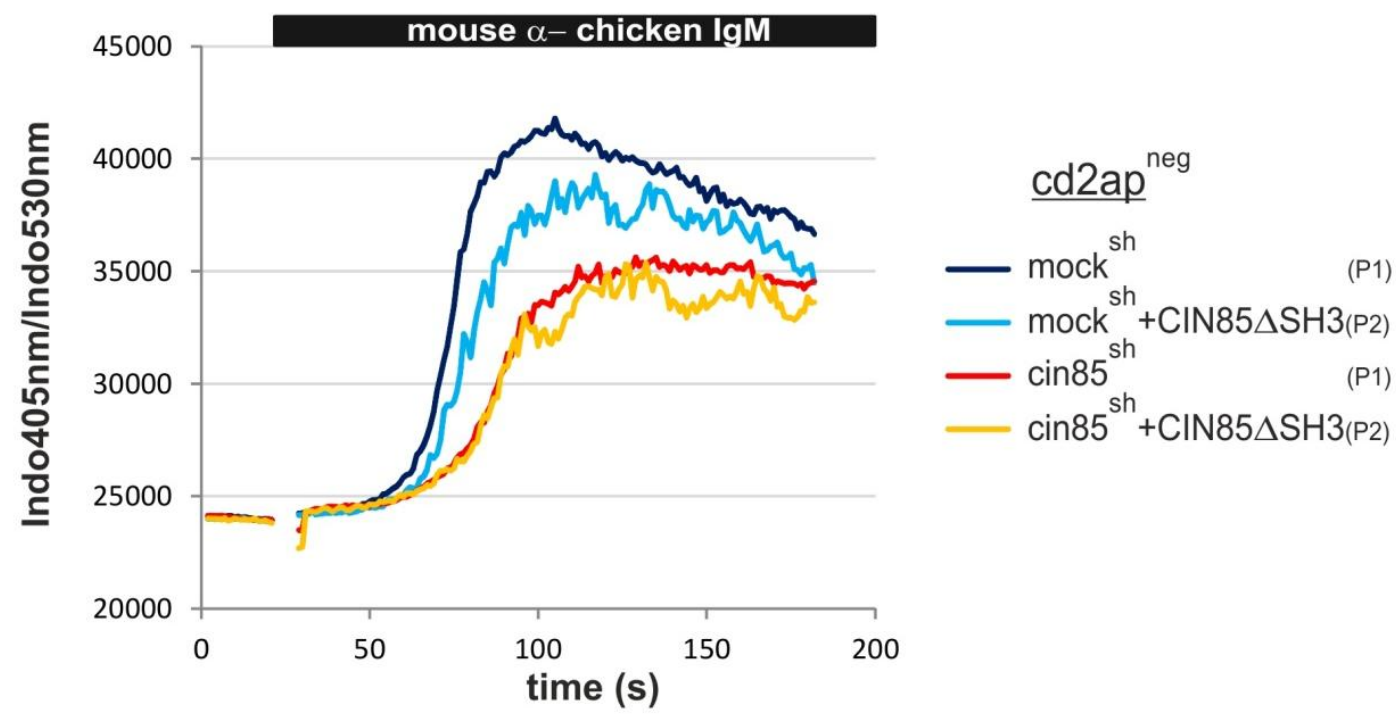

Figure 3.15: The SH3 domains of CIN85 are necessary domains for $\mathrm{Ca}^{2+}$ mobilisation in $\mathrm{BCR}$ stimulated DT40 B cells.

A) Wild-type (lanes 1 and 2) or CD2AP-deficient (lanes 3 and 4) DT40 B cells expressing either shRNA against $\operatorname{cin} 85$ mRNA (cin85 ${ }^{\text {sh }}$, lanes 2 and 4 ) or control shRNA (mock ${ }^{\text {sh }}$, lane 1 and 3 ) were transduced with constructs coding for Citrine-tagged $\mathrm{CIN} 85 \Delta \mathrm{SH} 3$ and cleared cellular lysates were subjected to SDS-PAGE and analysed by immunoblotting with antibodies recognising CIN85 (short or long exposure), chicken CD2AP or actin (upper two, middle and lower panel, respectively). B) Representative histogram of flow cytometric analysis of cells described in A) showing the gates for Citrine-negative (P1) or Citrine-positive (P2) populations. C) and D) Flow cytometric analysis of BCRinduced $\mathrm{Ca}^{2+}$ mobilisation of cells described in A) after gating for Citrine-CIN85 $\triangle \mathrm{SH} 3$ positive (light blue and yellow lines) or negative cells (dark blue and red lines) in either wild-type $\mathrm{C}$, wt) or cd2ap ${ }^{-1}$ DT40 B cells $\left(D, c d 2 a p^{\text {neg }}\right)$. Apparent molecular weights of protein standards are indicated on the left in $\mathrm{kDa}$. 
Expression of $\operatorname{cin} 85 \triangle C C$ after shRNA mediated targeting of endogenous cin85 mRNA could not restore full $\mathrm{Ca}^{2+}$ signalling in wild-type DT40 B cells (compare figure 3.16C, red and yellow lines). Performing the same experiment on $\mathrm{cd}_{2} \mathrm{ap}^{-/}$genetic background revealed that the BCR-induced $\mathrm{Ca}^{2+}$ signal of $c d 2 a p^{\text {neg }} / \mathrm{cin}^{2} 5^{\text {sh }}+\mathrm{CIN85} \Delta \mathrm{CC}$ DT40 B cells did not revert the full $\mathrm{Ca}^{2+}$ mobilisation competence compared to $\mathrm{cd} 2 \mathrm{ap} \mathrm{p}^{\text {neg }} / \mathrm{mock}^{\mathrm{sh}}+\mathrm{CIN} 85 \Delta \mathrm{CC}$ control $\mathrm{B}$ cells. However, a slightly increased signal could be observed in cd2ap ${ }^{\text {neg }} / \mathrm{cin}^{2} 5^{\text {sh }}+\mathrm{CIN} 85 \Delta$ CC DT40 B cells (figure 3.16D, yellow line).

A)

B)
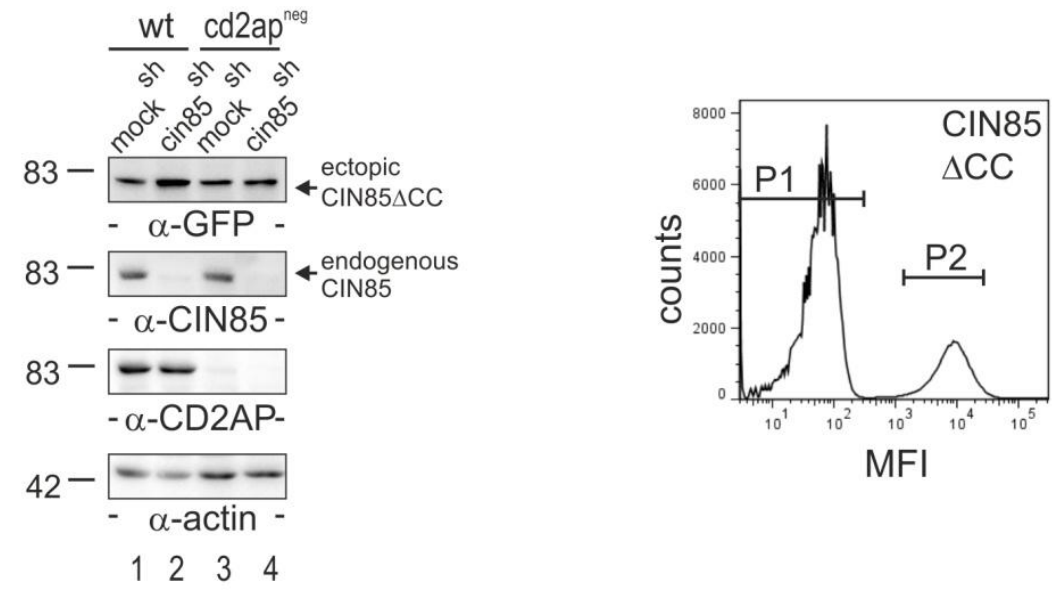

(figure continues on next page) 
C)

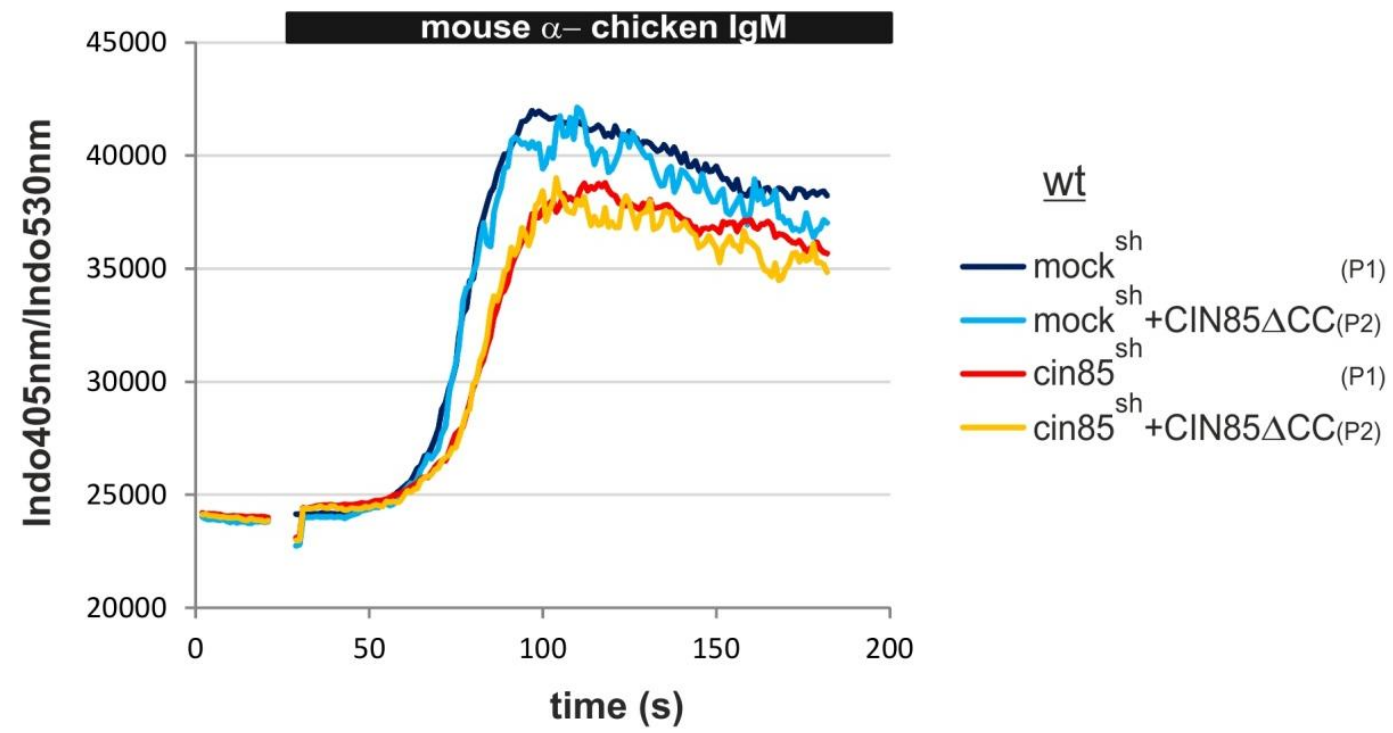

D)

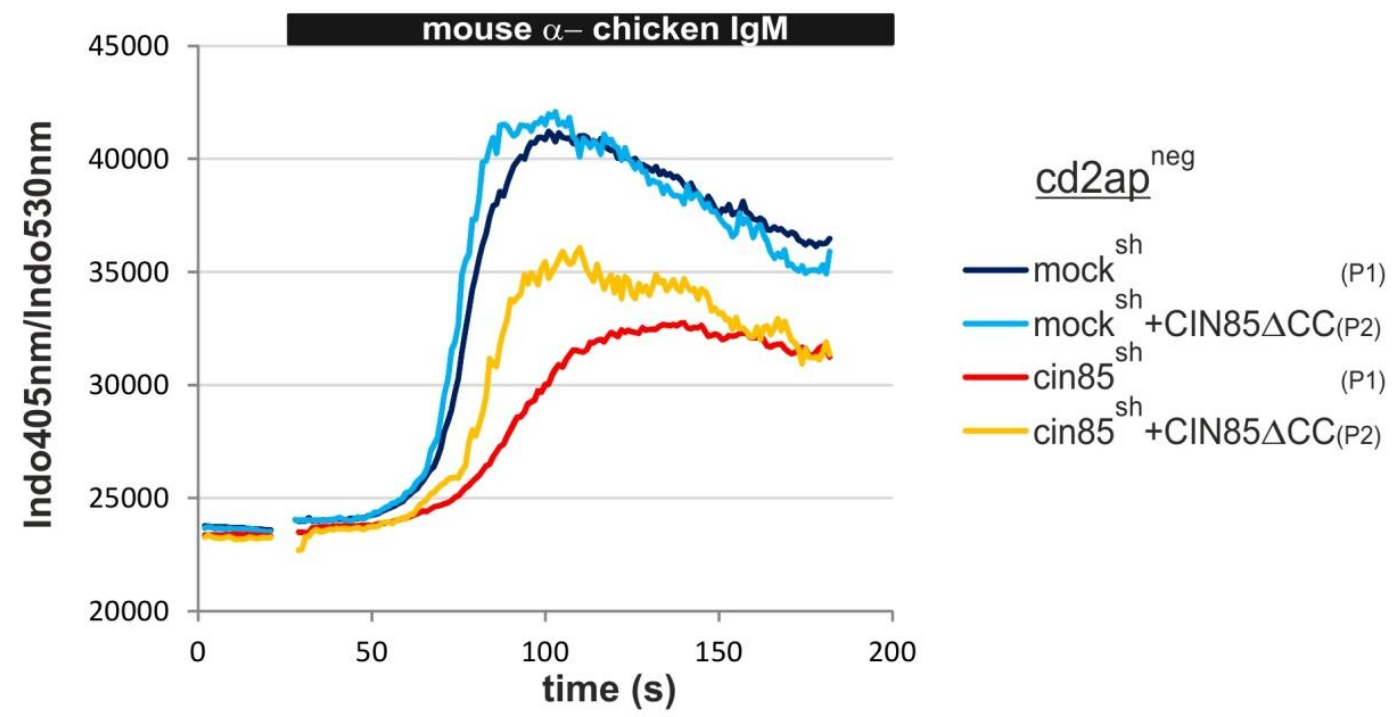

Figure 3.16: The coiled coil domain of CIN85 exerts a function in $\mathrm{BCR}$-induced $\mathrm{Ca}^{2+}$ flux.

A) Wild-type or cd2ap ${ }^{-1}$ DT40 B cells were retrovirally transduced to express an shRNA against cin85 mRNA (cin85 $5^{\text {sh }}$, lanes 2 and 4) or a control shRNA (mock ${ }^{\text {sh }}$, lanes 1 and 3) prior to transfection with constructs coding for Citrine-tagged CIN85 $\mathrm{CC}$. Cleared cellular lysates were subjected to SDSPAGE followed by immunoblotting with antibodies against GFP, CIN85, chicken CD2AP or actin (from top to bottom). B) Representative histogram obtained by flow cytometry of cells described in A). Gates reflect the Citrine-negative or Citrine-positive populations separately analysed in $C$ ) and D). C) and D) Flow cytometric analysis of $\mathrm{BCR}$-induced $\mathrm{Ca}^{2+}$ mobilisation of cells described in A) after gating for Citrine-CIN85 $\triangle \mathrm{CC}$ positive (light blue and yellow lines) or negative cells (dark blue and red lines) in either wild-type (C, wt) or cd2ap \% DT40 B cells (D, cd2ap $\left.{ }^{\text {neg }}\right)$. Apparent molecular weights of protein standards are indicated on the left in $\mathrm{kDa}$. 
In line with the hypothesis that membrane recruitment of CIN85 might be a crucial step for its positive regulatory function in BCR-induced $\mathrm{Ca}^{2+}$ mobilisation, the $\mathrm{SH} 3$ and coiled coil domains of CIN85 were found to be indispensable for both plasma membrane localisation and a full $\mathrm{Ca}^{2+}$ response in BCR-stimulated $\mathrm{B}$ lymphocytes.

\subsection{CIN85 colocalises with BCR-containing microclusters}

In response to membrane-bound antigen most BCR-proximal effector proteins colocalise to clusters of BCRs in the plasma membrane - the BCR-containing microclusters (Sohn et al. 2008; Weber et al. 2008). We were interested to see if CIN85 or CD2AP can be found at this site of most proximal BCR signalling. Therefore total internal reflection microscopy (TIRF) of cells settled on planar lipid bilayers (Fleire et al. 2006) was performed in collaboration with Tim Schnyder and Dr. Facundo Batista (Lymphocyte Interaction Laboratory, Cancer Research UK, London, UK). The planar lipid bilayer contained fluorophor-coupled anti-BCR antibodies that were able to freely diffuse in the lipid bilayer and stimulate the settling $B$ cell. Upon contact with the anti-BCR-loaded bilayer, small BCR-containing microclusters are formed. The cells spread over the lipid bilayer before they contract and gather antigen in a central cluster. TIRF microscopy allowed investigation of the colocalisation of fluorescent anti-IgM antibodies with fluorescently-tagged CIN85 or CD2AP during this B cell response.

\subsubsection{CIN85 colocalises with BCR-containing microclusters independent of SLP65}

TIRF microscopy of wild-type DT40 B cells expressing Citrine-tagged CIN85 was performed on anti-lgM-loaded lipid bilayers. Figure 3.17A shows the localisation of CIN85 (left images, green) in comparison to fluorescent anti-lgM (middle images, red) and merged images from both fluorophores at the beginning as well as at the end of the BCR-induced B cell response (upper and lower panel, respectively). CIN85 colocalised with the microclusters after engagement of the BCR (figure 3.17A, upper panel), remained localised to BCR-clusters during contraction of the cells and was finally part of the central cluster of BCRs (figure 3.17A, lower panel). To follow the complete course of BCR-induced micro - and central cluster formation see supplemental movie 2 in (Oellerich et al. 2011).

To test whether the preformation of CIN85 with SLP65 is required for the colocalisation of CIN85 with BCR-containing microclusters, s/p65 DT40 B cells expressing Citrine-tagged CIN85 were investigated next. The recruitment of CIN85 to signalling-competent microclusters and its localisation to the immunological synapse was independent of SLP65, since colocalisation with the BCR was also evident in s/p65 ${ }^{-1-}$ DT40 B cells (figure 3.17B). 
The distribution of CD2AP with respect to the BCR was analysed for Citrine-tagged CD2AP expressed in cd2ap \% DT40 B cells. Formation of the microclusters and the central BCR cluster seemed to occur normally in the cd2ap \% DT40 B cells as determined by the anti-IgM antibodies initially distributed and later pooled in a central cluster (figure 3.17C). However, rather few CD2AP molecules colocalised with the BCR-containing microclusters over time (also compare supplementary movie 4 in (Oellerich et al. 2011)).

A)

DT40 B cells

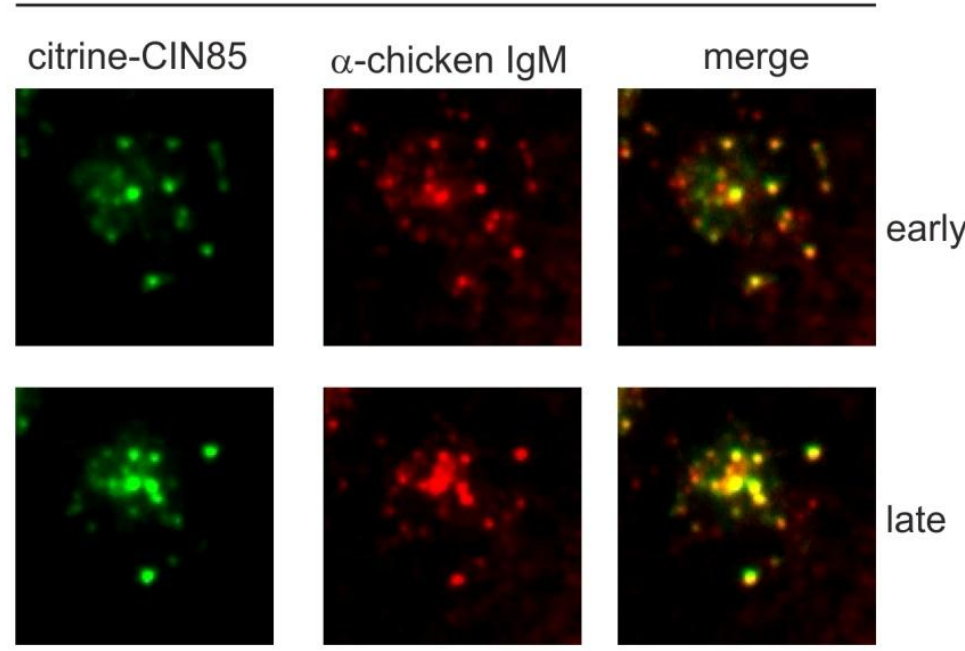

B) slp65 ${ }^{-/}$DT40 B cells
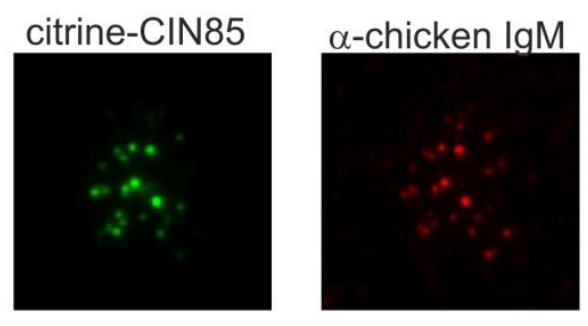

merge

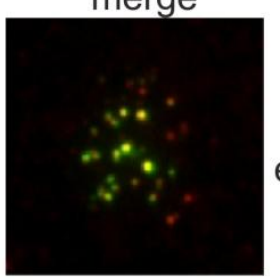

early
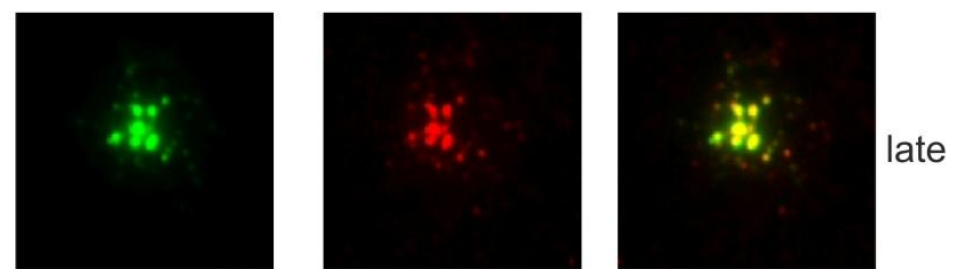

(figure continues on next page) 
C)

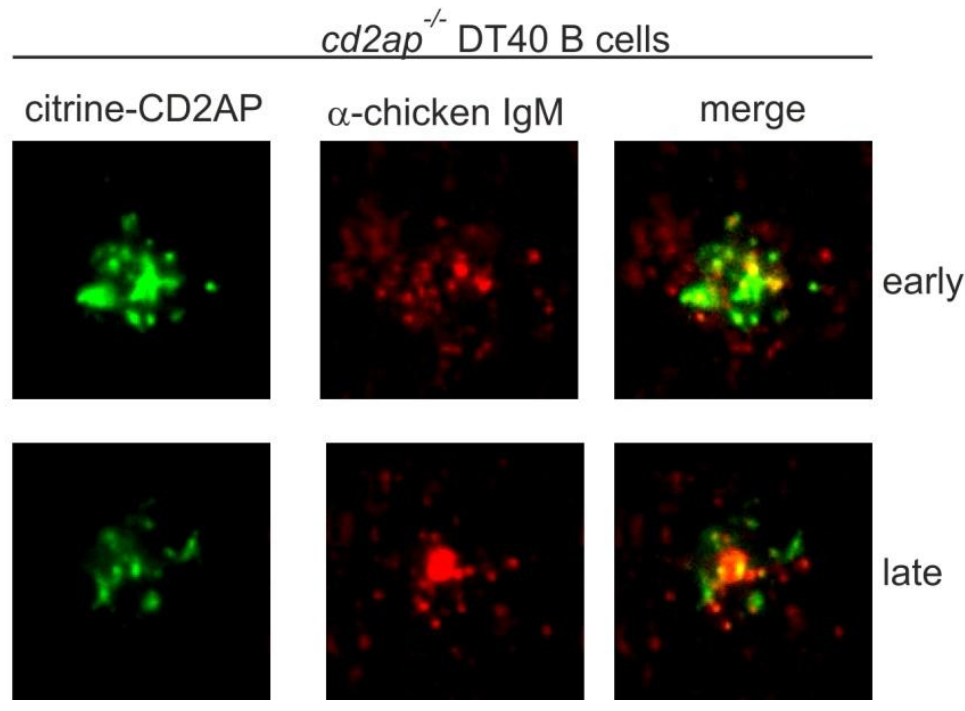

Figure 3.17: CIN85 colocalises with BCR-containing microclusters independent of SLP65.

A) Wild-type or B) s/p65 $\%$ DT40 B cells were retrovirally transduced to express Citrine-tagged CIN85 or C) CD2AP-deficient DT40 B cells were reconstituted with Citrine-tagged chicken CD2AP and settled on planar lipid bilayers containing fluorescently labelled anti-chicken $\lg M$ as a BCR stimulus. Colocalisation (Merge) of CD2AP or CIN85 (green) and anti-chicken IgM (red) was analysed by TIRF microscopy in collaboration with Tim Schnyder and Facundo Batista (Lymphocyte Interaction Laboratory, Cancer research UK, London, UK) as described in (Oellerich et al. 2011). The upper panels reflect TIRF microscopic images acquired early after settling of the cell, while the lower panels show localisation at the time BCRs are gathered into a central cluster. An additional demonstration of this experiment can be found in figure 5 and supplementary movies 2-4 in (Oellerich et al. 2011).

Recruitment of CIN85 to BCR-containing microclusters observed by TIRF microscopy could be further supported by biochemical means. Figure 3.18 shows an immuno purification of surface bound IgM from wild-type DT40 cells expressing either Citrine-tagged versions of CD2AP or CIN85 lysed under mild-detergent conditions. Unlike in unstimulated cells, after stimulation of the BCR for 2 minutes CIN85 was copurified with the surface BCR (lane 9). Over time the association seemed to get lost, but the amount of precipitated IgM decreased as well (lanes 4, 5, 10 and 11). This is possibly due to BCR aggregates at the cell surface forming insoluble precipitates that are pelleted after centrifugation. CD2AP did not precipitate with the BCR upon stimulation, but a weak association was also observed in unstimulated cells. 


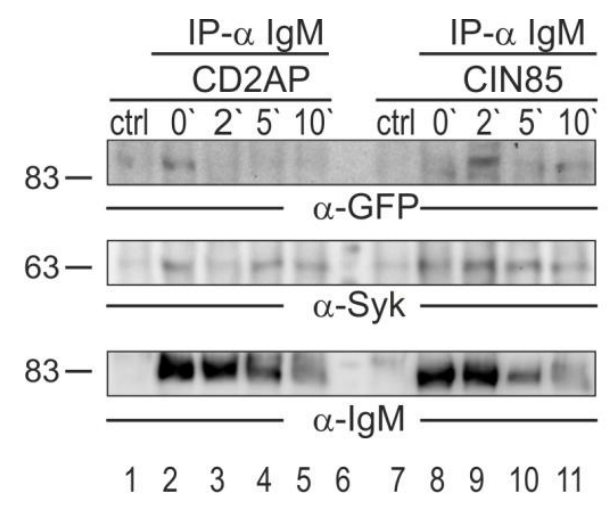

Figure 3.18: CIN85 associates with surface IgM. Wild-type DT40 B cells expressing either CD2AP or CIN85 as fusion with a Citrine-tag (lanes 1-5 or lanes 6-10, respectively) were incubated with antichicken IgM for indicated times at $37^{\circ} \mathrm{C}$ or on ice ( $0^{\circ}$, lanes 2 and 7$)$, washed and lysed. The BCR was precipitated using anti-mouse IgM antibodies coupled to ProteinA/G Sepharose. As a control cleared cellular lysates were incubated with beads only (ctrl., lanes 1 and 6). Proteins were analysed by SDS-PAGE followed by immunoblotting with anti-GFP, anti-Syk or anti-chicken IgM antibodies (upper, middle and lower panel, respectively). Apparent molecular weights of protein standards are indicated on the left in $\mathrm{kDa}$.

The microscopic analysis revealed for the first time a localisation of CIN85 within BCRcontaining microclusters, which was also evident in the absence of SLP65. The biochemical experiment likewise indicated an association of CIN85 with the BCR.

\subsubsection{BCR internalisation is not affected by reduced cin85 expression}

CIN85 colocalised with BCR clusters at the immunological synapse, which is the site of antigen uptake (Batista et al. 2001) and is known for its ability to support internalisation of e.g. epidermal growth factor receptors (Soubeyran et al. 2002). Thus, it was hypothesised that CIN85 also plays a role in BCR internalisation of BCR-activated B cells.

This was tested by flow cytometric determination of surface $\lg \mathrm{M}$ in wild-type or CD2APdeficient DT40 B cells transfected with an shRNA against cin85 mRNA (figure 3.19). The efficiency of arrested cin85 expression was determined via immunoblotting with anti-CIN85 antibodies (figure 3.19A, lanes 2 and 4). Internalisation of BCR molecules was induced with anti-lgM antibodies at $37^{\circ} \mathrm{C}$. After incubation for the indicated time points and washing away of the primary anti-lgM antibody, residual surface BCRs were stained with a secondary, fluorescently-labelled antibody. With this setup no intracellular, internalised or newly synthesised BCRs should be detected. Figure 3.19B and C shows that after anti-lgM crosslinking the BCR was internalised resulting in decreasing BCR molecules at the surface over time. Stimulation of the B cells for 20 minutes led to a decrease of surface BCR of over $80 \%$ compared to unstimulated cells (figure 3.19B, 0 and $20 \mathrm{~min}$ ). Reduced expression of cin85 had no effect on BCR internalisation at all time points analysed. Likewise, arrest of cin85 expression on the cd2ap $\%$ genetic background did not alter the kinetics of BCR internalisation as compared to cd2ap neg/mock ${ }^{\text {sh }}$ DT40 B cells (figure 3.19C). However, BCR internalisation was increased in cd2ap ${ }^{-1} \mathrm{~B}$ cells, in which already $57 \%$ of all surface BCRs 
were internalised after $5 \mathrm{~min}$, compared to $35 \%$ surface BCR in wild-type DT40 B cells. Thus, a function or functional redundancy of CIN85 and CD2AP in BCR internalisation could not be identified in this setup.

A)

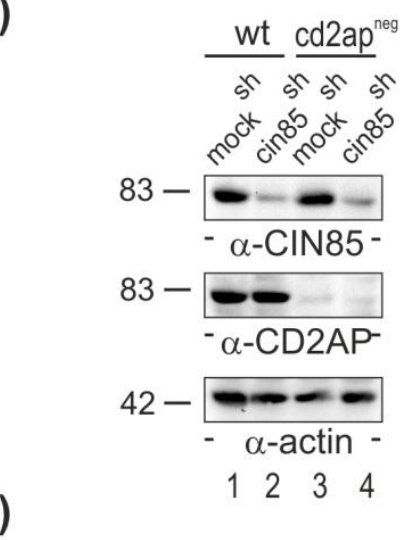

B)

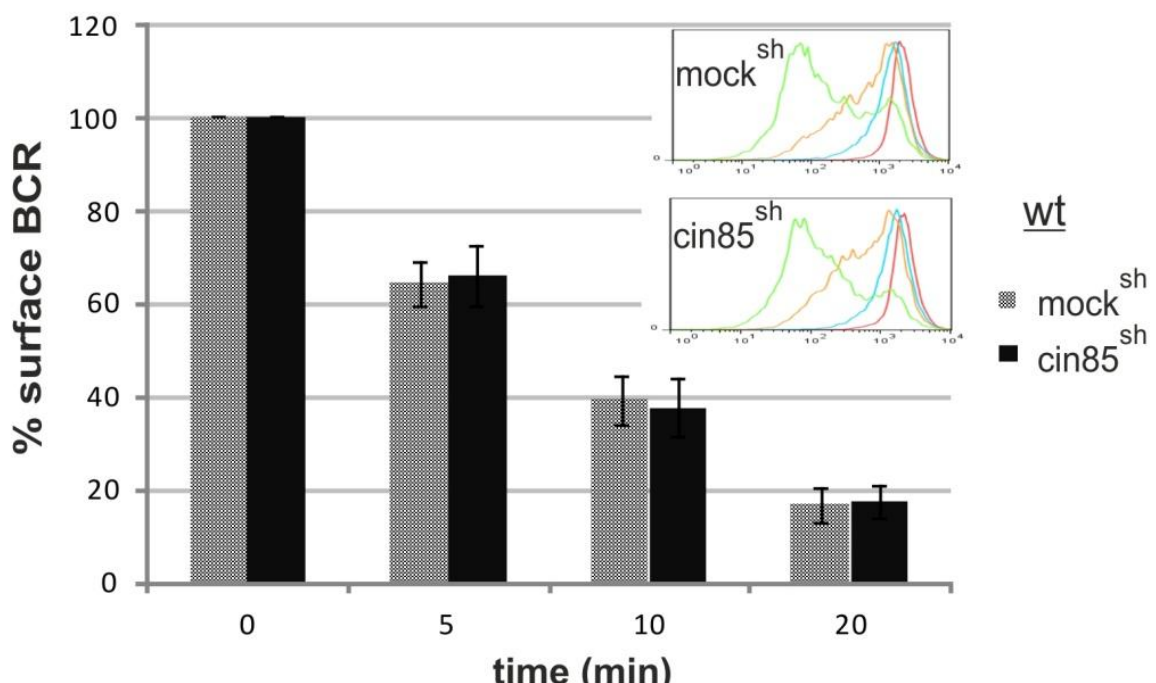

C)

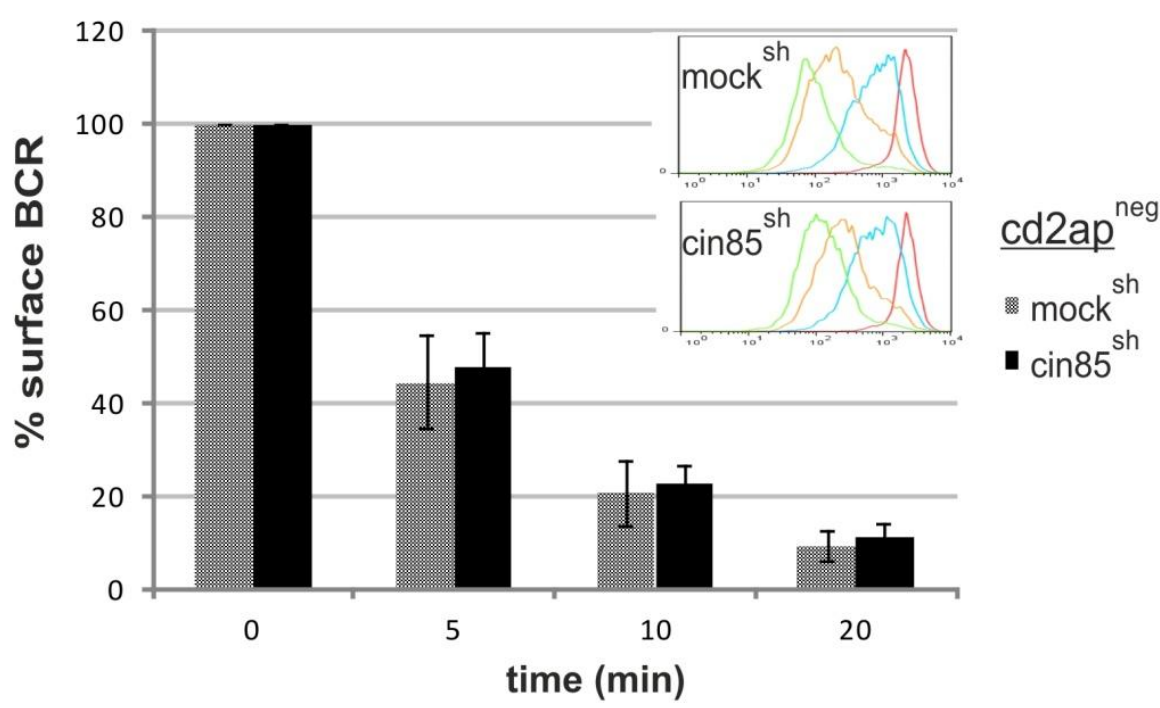

Figure 3.19: Reduction of cin85 expression has no influence on BCR internalisation.

(legend on next page) 
Figure 3.19: Reduction of cin85 expression has no influence on BCR internalisation

A) Wild-type (wt) or CD2AP-deficient (-/-) DT40 B cells were retrovirally transduced to express shRNA against chicken CIN85 (cin85 ${ }^{\text {sh }}$, lanes 2 and 4) or control shRNA (mock ${ }^{\text {sh }}$, lanes 1 and 3 ) and cleared cellular lysates were subjected to SDS-PAGE followed by immunoblotting with antibodies against CIN85, chicken CD2AP or actin (upper, middle or lower panel, respectively) or B) wild-type or C) CD2AP-deficient $\left(\mathrm{cd}_{2} a \mathrm{p}^{\text {neg }}\right.$ ) DT40 B cells described in A) were analysed for surface IgM by flow cytometry for indicated time points (cin85 $5^{\text {sh }}$, black bars or mock $^{\text {sh }}$, shaded bars, respectively). The diagram shows the percentage of surface BCR determined from the mean fluorescence intensity of $\mathrm{Cy}^{\mathrm{TM}} 5$-conjugated $\left.\mathrm{F}(\mathrm{ab})_{2}\right)_{2}$ fragment bound to chicken $\operatorname{lgM}$ via M4. B) and C) inlay Histograms of fluorescently labelled surface $\operatorname{lgM}$ of cells described in A) before or 5, 10 or $20 \mathrm{~min}$ after stimulation (red, blue, orange or green line, respectively). Standard deviations were calculated from three independent experiments.

\subsection{Lyn, but not Syk kinase activity is required for BCR-induced plasma membrane translocation of CIN85}

\subsubsection{The BCR-induced recruitment of CIN85 to the plasma membrane requires Lyn, but not Syk}

To further investigate the mechanisms underlying the membrane recruitment and presumably BCR microcluster colocalisation of CIN85 in activated B cells, I focussed on the BCR-proximal kinases Lyn and Syk. Although Lyn is important for the phosphorylation of the ITAMs, Lyn-deficient DT40 B cells are able to induce a delayed $\mathrm{Ca}^{2+}$ response (Takata et al. 1994). In order to decipher a Lyn specific function, I wanted to abolish residual BCR signalling in $l y n^{-1} \mathrm{~B}$ cells by expression of a dominant-negative variant of Lyn. In this Lyn variant the lysine $(K)$ residue at position 275 was replaced by an arginine $(R)$, which results in loss of Lyn kinase-activity (Corey et al. 1998).

lyn $^{-1}$ or lyn ${ }^{-1}$ DT40 B cells reconstituted with HA-tagged wild-type Lyn or with the kinasedead variant of Lyn (Lyn KD), were subjected to $\mathrm{BCR}$-induced $\mathrm{Ca}^{2+}$ mobilisation analysis (figure 3.20A). Lyn-deficient DT40 B cells exhibit a delayed and decreased BCR-induced $\mathrm{Ca}^{2+}$ response (dark blue line). Expression of the kinase-dead variant of Lyn prevented the residual $\mathrm{Ca}^{2+}$ mobilisation in $/ y n^{-/}$DT40 $\mathrm{B}$ cells (dominant-negative), while reconstitution of $\mathrm{Iyn}^{-/-}$DT40 B cells with wild-type Lyn exhibited a fast and strong BCR-induced $\mathrm{Ca}^{2+}$ response (red and light blue line, respectively).

With the established Lyn-transfectants, I analysed BCR-induced CIN85 membrane recruitment by confocal microscopy. Expression of HA-tagged Lyn variants and Citrinetagged CIN85 was confirmed by immunoblotting (figure 3.20B. left). In lyn ${ }^{\star-}$ DT40 B cells expressing the kinase-dead variant of Lyn, membrane translocation of CIN85 could not be observed after BCR cross-linking (figure 3.20B, right, upper panel). Lyn-deficient DT40 B 
cells transfected with wild-type Lyn served as a positive control. Indeed, in these cells CIN85 was recruited to the plasma membrane again (figure 3.20B, lower panel, right image).

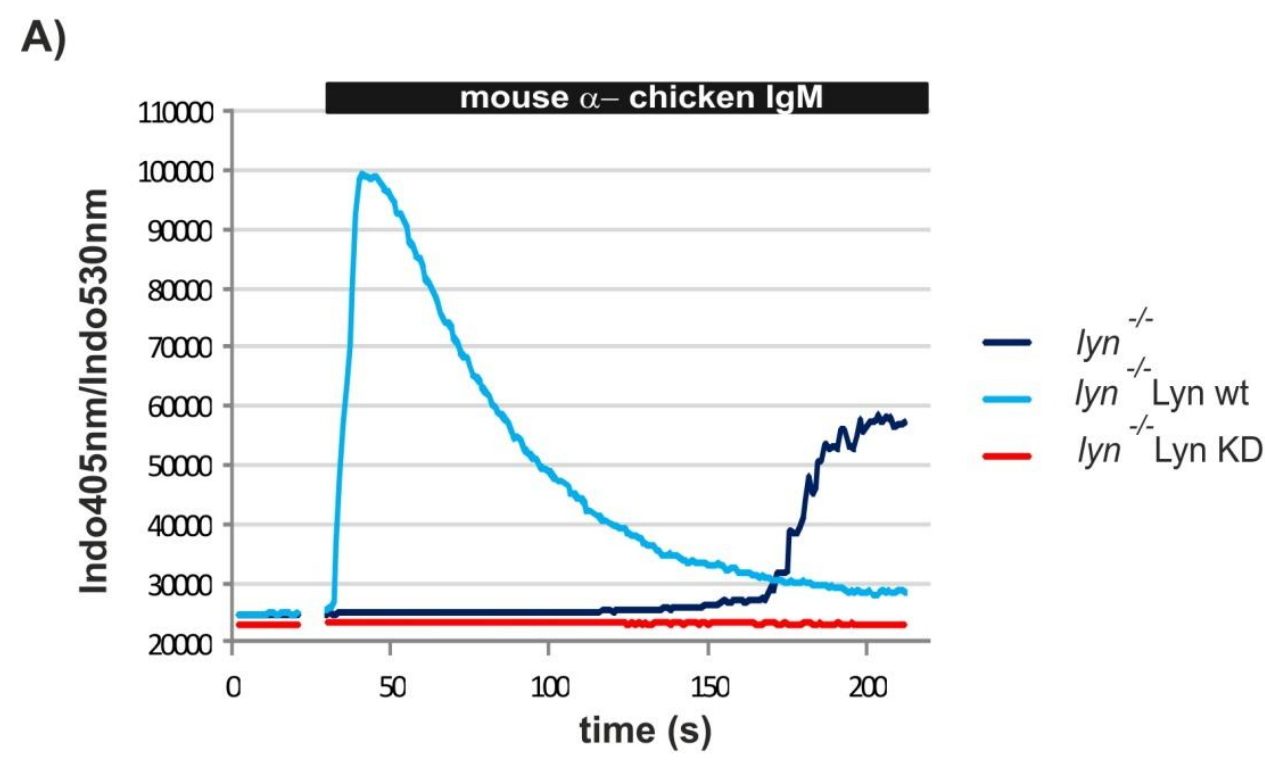

B)

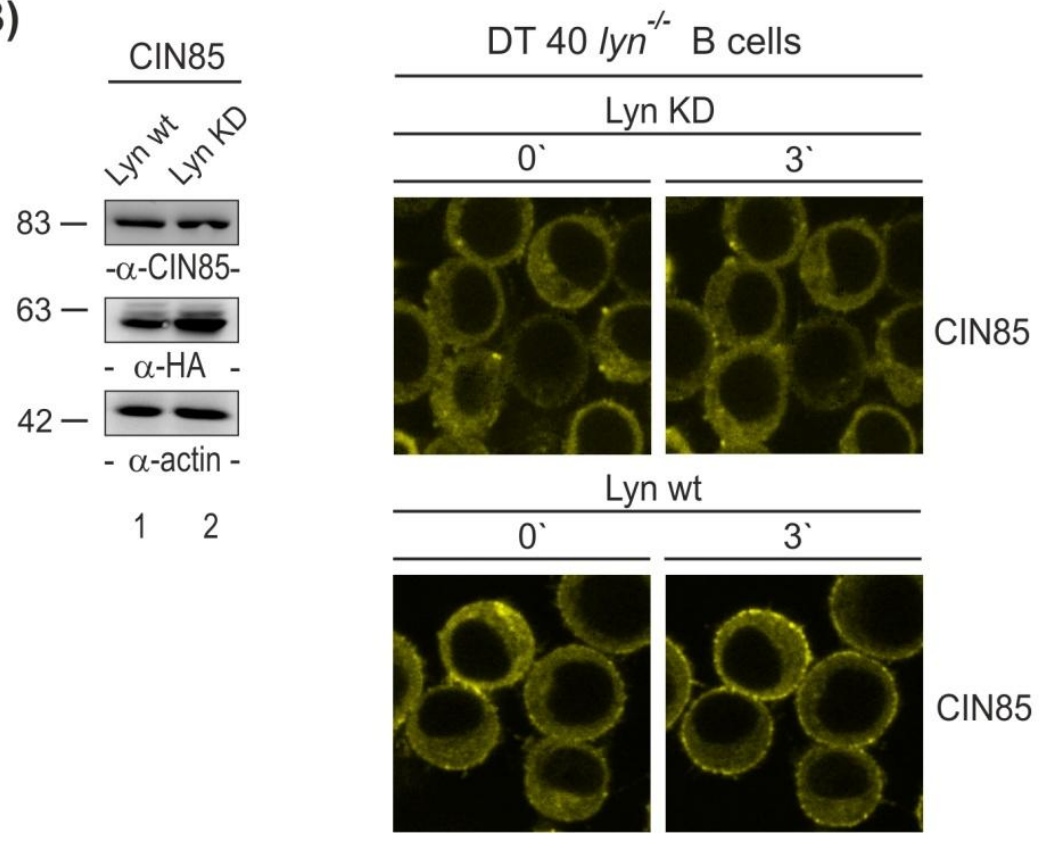

(figure continues on next page) 
C)

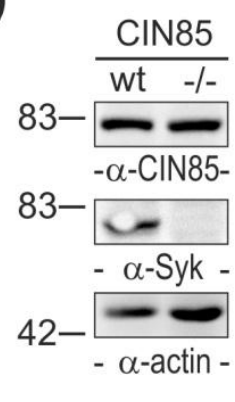

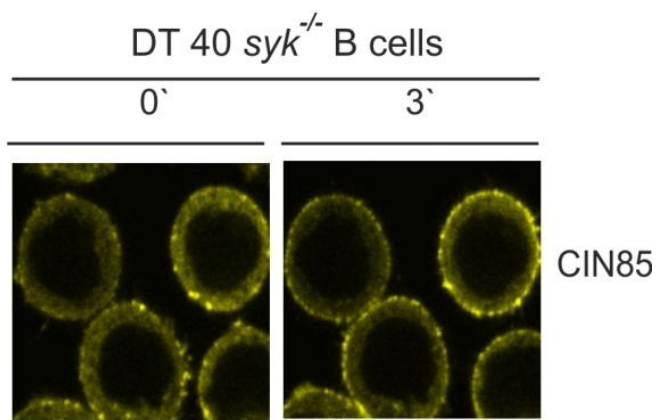

12

Figure 3.20: The BCR-induced membrane recruitment of CIN85 requires Lyn, but not Syk.

A) Lyn-deficient DT40 B cells (dark blue line) or $l y n^{-/}$reconstituted with HA-tagged wild-type (Lyn wt, light blue line) or a kinase-dead variant of Lyn ( $\mathrm{Lyn} K \mathrm{KD}$, red line) were subjected to $\mathrm{BCR}$-Induced $\mathrm{Ca}^{2+}$ mobilisation analysis. B) Lyn wt or Lyn KD cells described in A) (right, middle and upper panel, respectively) or C) Syk-deficient cells were retrovirally transduced to express Citrine-tagged CIN85. Cleared cellular lysates of cells were subjected to SDS-PAGE before immunoblotting with anti-HA, anti-Syk, anti-CIN85 or anti-actin antibodies (left panels) or were analysed by confocal laser scanning microscopy in the absence of (left images) or presence of BCR stimulation (right images).

In syk ${ }^{-1}$ DT40 B cells CIN85 attached to the plasma membrane upon BCR stimulation, but was a bit enriched prior to BCR triggering $\left(M_{\text {stim. }}=1.15\right.$, figure 3.20C). Two independent experiments of CIN85 membrane recruitment in syk ${ }^{-/}$DT40 B cells are shown in table 3.2. The first shows a small reduction in the fold change of CIN85 membrane recruitment, while in the second experiment CIN85 translocation was increased when compared to a fold-change of CIN85 membrane recruitment in wild-type DT40 B cells of $($ CIN85 $)=1.28$. On average this indicates that the enzymatic activity of Syk was not required for membrane translocation of CIN85.

Table 3.2 Quantification of plasma membrane localisation of CIN85 in different DT40 B cell lines

\begin{tabular}{ccccc}
\hline DT40 B cell specimen & $\mathbf{M}_{\text {rest. }}$ & $\mathbf{M}_{\text {stim. }}$ & fold-change & number of cells \\
\hline CIN85 in lyn ${ }^{-/-}$Lyn KD & $\mathbf{1 . 0 0} \pm 0.02$ & $\mathbf{0 . 9 9} \pm 0.03$ & $\mathbf{1 . 0 0} \pm 0.06$ & $\mathrm{n}=42$ \\
CIN85 in lyn ${ }^{-/}$Lyn wt & $\mathbf{1 . 0 9} \pm 0.09$ & $\mathbf{1 . 5 0} \pm 0.05$ & $\mathbf{1 . 3 9} \pm 0.08$ & $\mathrm{n}=48$ \\
CIN85 in syk & $\mathbf{1 . 1 8} \pm 0.07$ & $\mathbf{1 . 4 1} \pm 0.21$ & $\mathbf{1 . 2 1} \pm 0.14$ & $\mathrm{n}=82$ \\
CIN85 in syk & $\mathbf{1 . 1 5} \pm 0.07$ & $\mathbf{1 . 5 6} \pm 0.09$ & $\mathbf{1 . 3 7} \pm 0.07$ & $\mathrm{n}=116$ \\
\hline
\end{tabular}

Relative membrane localisation (M) of Citrine-tagged CIN85 expressed in DT40 B cells. M reflects the mean fluorescence intensity at the plasma membrane normalised to the fluorescence intensity in the cytosol in either unstimulated $\left(\mathrm{M}_{\text {rest. }}\right)$ or 3min BCR-stimulated DT40 B cells $\left(\mathrm{M}_{\text {stim. }}\right)$. The fold-change equals the mean of the ratios of $M_{\text {stim. }}$ and $M_{\text {rest. }}$ calculated for each cell. Quantification ( $\pm S D$ ) of replicates of one independent experiment is shown. See section 2.2.4 for details. 


\subsubsection{Membrane recruitment of CIN85 after BCR stimulation is independent of CD2AP or Grb2}

Previous experiments have shown that the coiled coil and SH3 domains of CIN85, mediating protein-protein interactions, are necessary for its membrane recruitment raising the question whether CIN85 membrane recruitment in BCR-activated B cells depends on its interaction partners CD2AP or Grb2 (Kirsch et al. 1999; Borinstein et al. 2000; Watanabe et al. 2000). I analysed localisation of Citrine-tagged CIN85 in either cd2ap $\%$ or grb2 ${ }^{\%}$ DT40 B cells by confocal laser scanning microscopy (figure 3.21).

Interestingly, in CD2AP-deficient DT40 B cells CIN85 is enriched at the plasma membrane prior to BCR-stimulation reflected by an $\mathrm{M}_{\text {rest. }}\left(\mathrm{CIN85}\right.$ in $\left.c d 2 a p^{--}\right)=1.22$, thus was higher than in all other DT40 B cells examined so far. However, after BCR cross-linking CIN85 still translocated to the plasma membrane in the absence of CD2AP (figure 3.21, upper panel, right image) with a normal fold-change of 1.34. Likewise, Grb2-deficiency did not abolish BCR-induced membrane recruitment of CIN85 (figure 3.21, lower panel and table 3.3).

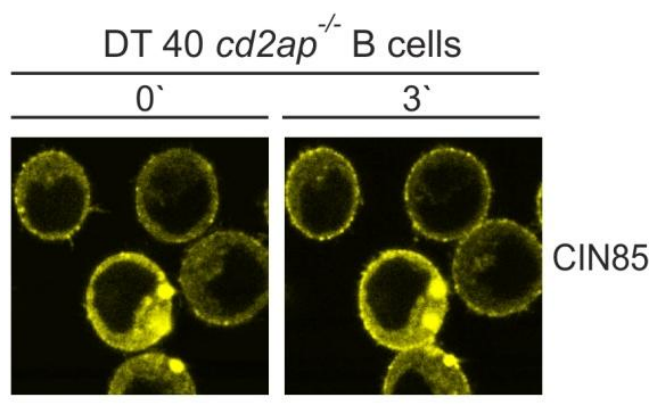

DT $40 \mathrm{grb}^{-/-}$B cells

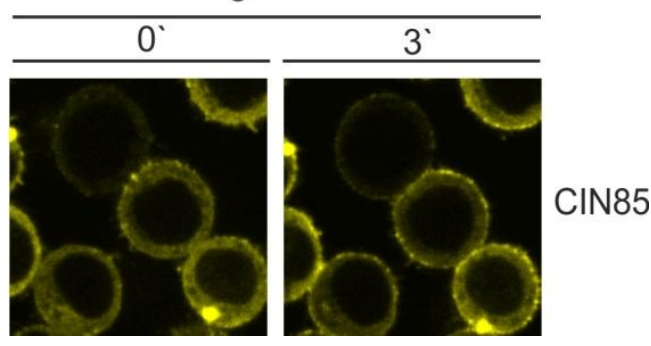

Figure 3.21: BCR-induced membrane recruitment of CIN85 is independent of CD2AP or Grb2.

Confocal laser scanning microscopy of cd2ap (upper panel) or grb2 $\%$ (lower panel) DT40 B cells expressing Citrine-tagged human CIN85 before or $3 \mathrm{~min}$ after BCR-stimulation (left and right images, respectively).

Table 3.3 Quantification of plasma membrane localisation of CIN85 in cd2ap ${ }^{\%}$ or grb2 ${ }^{\%}$ DT40 B cell lines before and after BCR stimulation

\begin{tabular}{ccccc}
\hline DT40 B cell specimen & $\mathbf{M}_{\text {rest. }}$ & $\mathbf{M}_{\text {stim. }}$ & fold-change & number of cells \\
\hline cd2ap $^{-/-}$ & $\mathbf{1 . 2 2} \pm 0.04$ & $\mathbf{1 . 6 2} \pm 0.08$ & $\mathbf{1 . 3 4} \pm 0.05$ & $\mathrm{n}=67$ \\
grb2 $^{-/}$ & $\mathbf{1 . 1 0} \pm 0.02$ & $\mathbf{1 . 4 1} \pm 0.09$ & $\mathbf{1 . 2 9} \pm 0.1$ & $\mathrm{n}=74$
\end{tabular}

Relative membrane localisation (M) of Citrine-tagged CIN85 expressed in cd2ap ${ }^{-1}$ or grb2 $^{\%}$ DT40 B cells. $M$ reflects the mean fluorescence intensity at the plasma membrane normalised to the fluorescence intensity in the cytosol in either unstimulated $\left(\mathrm{M}_{\text {rest. }}\right)$ or 3min BCR-stimulated DT40 B cells $\left(M_{\text {stim. }}\right)$. The fold-change equals the mean of the ratios of $M_{\text {stim. }}$ and $M_{\text {rest. }}$ calculated for each cell. Quantification $( \pm S D)$ of at least triplicates representative of two independent experiments is shown. See section 2.2.4 for details. 


\subsection{BCR-targeted SLP65 functions independent of CIN85/CD2AP in BCR- triggered $\mathrm{Ca}^{2+}$ mobilisation}

\subsubsection{The TIRAP PIP 2 binding domain substitutes the function of the $\mathrm{N}$-terminus in SLP65_wt, but not in SLP65_M23.}

To test whether binding of CIN85/CD2AP to SLP65 serves the purpose of SLP65 plasma membrane localisation, I engineered chimeric proteins in which the first 48 amino acids of either SLP65_wt or the SLP65_M23 (no binding to CIN85/CD2AP) variant were replaced by the first 40 amino acids of human TIRAP. TIRAP is an adaptor protein in Toll-like receptor (TLR) signalling and contains a $\mathrm{PIP}_{2}$ binding domain, which allows its membrane localisation (Kagan and Medzhitov 2006). The anchoring function of the $\mathrm{PIP}_{2}$ binding domain can be inactivated upon exchange of the 4 lysines by alanines (Kagan and Medzhitov 2006; Hermann 2009). Fusion of the membrane-binding inactivated variant of TIRAP (mTIRAP) to SLP65 served as negative control. Figure 3.22A gives an overview of the SLP65 fusion proteins. s/p65 $\%$ DT40 B cells were retrovirally transduced to express either SLP65_wt as positive or SLP65_M23 as a negative control or the respective TIRAP chimeras

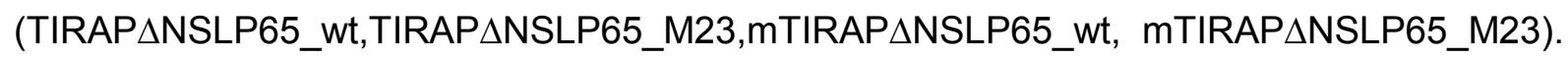
Anti-GFP immunoprecipitates of these TIRAP chimeras showed that the exchange of the SLP65 N-terminus did not alter the binding or lack of binding to CIN85/CD2AP (figure 3.22B, lanes 1 or 2 , respectively and data not shown).

Next, the s/p65 $1 / 2$ DT0 B cells transfectants were analysed for their competence in BCRinduced $\mathrm{Ca}^{2+}$ mobilisation (figure 3.22C). Reconstitution of s/p65 $\%$ DT40 B cells with wildtype SLP65 enabled the BCR-activated cells to mobilise $\mathrm{Ca}^{2+}$ in a profound way (dark blue curve), while deficiency in CIN85 or CD2AP binding strongly impaired SLP65 function (light blue curve, (Oellerich et al. 2011)). Exchange of the N-terminus of SLP65 with the TIRAP $\mathrm{PIP}_{2}$ binding domain resulted in the same $\mathrm{Ca}^{2+}$ mobilisation profile as wild-type SLP65 reconstituted cells (compare dark blue and red lines). In contrast, the TIRAP $\mathrm{PIP}_{2}$ binding domain was not sufficient to restore $\mathrm{Ca}^{2+}$ flux to SLP65_wt levels, when fused to the R-to-A variant of SLP65 (TIRAPANSLP65_M23, yellow line). The negative controls mTIRAP $\triangle N S L P 65 \_w t$ and mTIRAP $\triangle$ NSLP65_M23 could not mobilise $\mathrm{Ca}^{2+}$ (green lines). 
A)

N

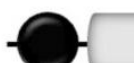

$\mathrm{N}$

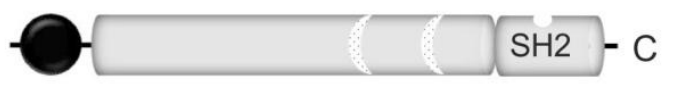

$\mathrm{N}$

m.

N

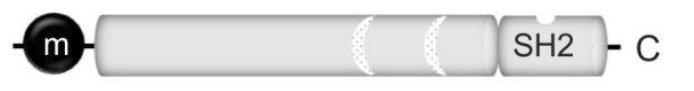

TIRAPANSLP65_wt

TIRAP $\Delta$ NSLP65_M23

mTIRAPANSLP65_wt

mTIRAPANSLP65_M23

B)

$$
\begin{array}{r}
\frac{\text { IP: } \alpha \text {-GFP }}{\text { TIRAP }} \\
\text { SLP65 } 54 \text { wt M23 } \\
83-\alpha-\text {-CIN85- } \\
12
\end{array}
$$

C)

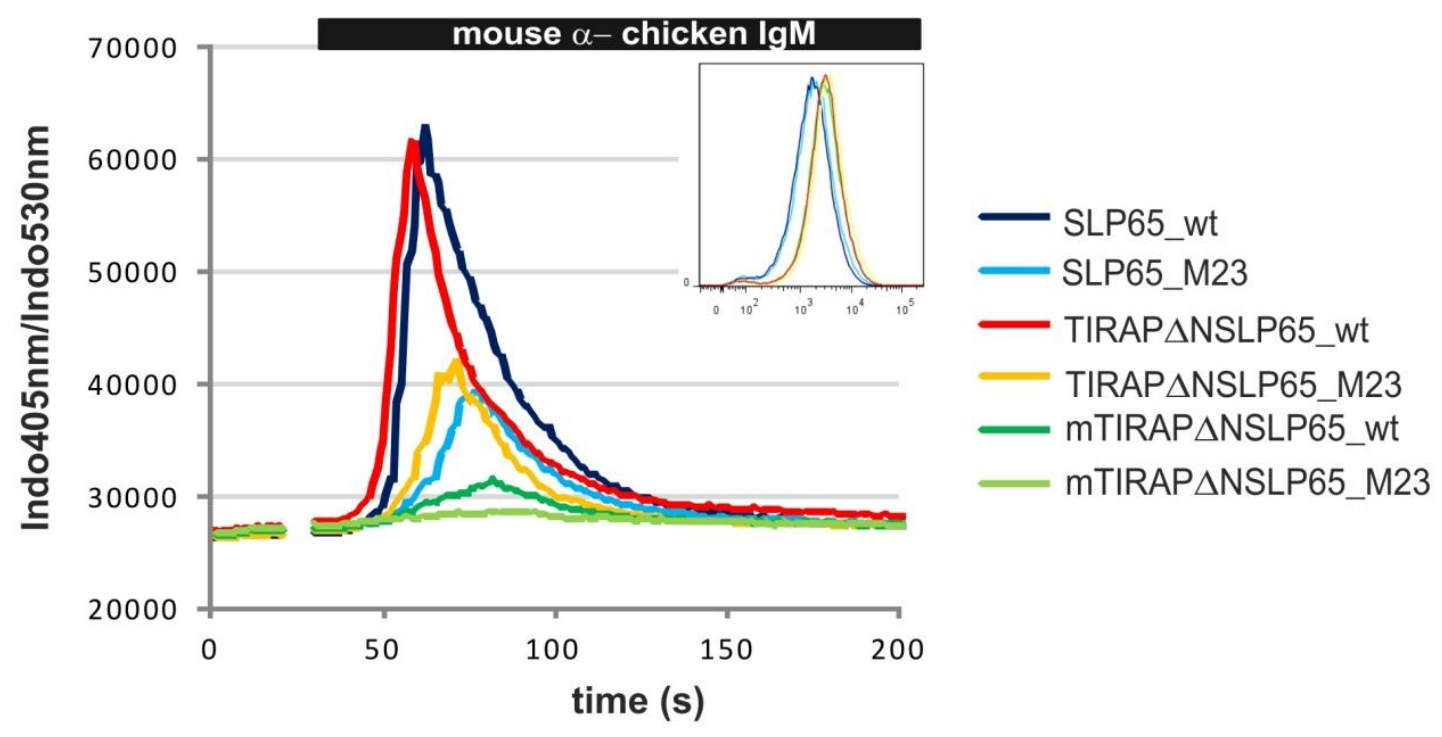

Figure 3.22: The TIRAP PIP $_{2}$ binding domain functionally substitutes the N-terminus of SLP65_wt, but not SLP65_M23.

A) Schematic representation of chimeric proteins of SLP65 in which the first 48 amino acids of SLP65 were replaced by the $\mathrm{PIP}_{2}$ binding domain of human TIRAP or a K-to-A variant (mTIRAP). The remaining two proline-arginine motifs were left intact (TIRAPANSLP65_wt, mTIRAPANSLP65_wt) or destroyed by R-to-A amino acid exchanges (TIRAPANSLP65_M23, mTIRAPANSLP65_M23). All chimeras harbour a N-terminal Citrine-tag (not shown) B) slp65 ${ }^{-1}$ DT40 B cells were retrovirally transduced to express the Citrine-tagged chimeras TIRAP $\triangle$ NSLP65_wt or TIRAP $\triangle N S L P 65 \_M 23$ and cleared cellular lysates subjected to anti-GFP immuno purifications followed by immunoblotting with antibodies recognising CIN85, chicken CD2AP or SLP65 (continued on next page) 
(legend to figure 3.22 continued) C) s/p65 ${ }^{-1}$ DT40 B cells expressing Citrine-tagged wild-type (SLP65_wt) or the R-to-A variant of SLP65 (SLP65_M23) or the respective TIRAP chimeras described in $A$ ) were analysed for $B C R$-induced $\mathrm{Ca}^{2+}$ mobilisation by flow cytometry using $1 \mu \mathrm{g} / \mathrm{ml} \mathrm{M} 4$. D) inlay Flow cytometric analysis of Citrine expression of cells analysed. Apparent molecular weights of protein standards are indicated on the left in $\mathrm{kDa}$.

By confocal laser scanning microscopy I analysed whether the engineered proteins were able to locate to the plasma membrane. Quantitative analyses as presented in table 3.4 showed that the TIRAP $\mathrm{PIP}_{2}$ binding domain did not anchor TIRAP $\triangle N S L P 65$ wt constitutively to the plasma membrane $\left(\mathrm{M}_{\text {rest. }}<1\right)$, but after $\mathrm{BCR}$ cross-linking the chimera translocated to the plasma membrane $\left(M_{\text {stim. }}\right.$ (TIRAP $\left.\Delta N S L P 65 \_w t\right)=1.13$ or foldchange=1.19). Incomprehensibly, the TIRAPANSLP65_M23 was not found at the plasma membrane in BCR-activated cells, respectively showed the same behaviour as mTIRAPANSLP65_wt, in which the TIRAP anchor is not supposed to bind to the plasma membrane.

Table 3.4: Quantification of the membrane localisation of the TIRAP-SLP65 chimeras

\begin{tabular}{ccccc}
\hline DT40 B cell specimen & $\mathbf{M}_{\text {rest. }}$ & $\mathbf{M}_{\text {stim. }}$ & fold-change & number of cells \\
\hline TIRAP $\triangle$ NSLP65_wt & $\mathbf{0 . 9 6} \pm 0.03$ & $\mathbf{1 . 1 3} \pm 0.03$ & $\mathbf{1 . 1 9} \pm 0.03$ & $\mathrm{n}=54$ \\
TIRAP $\triangle$ NSLP65_M23 & $\mathbf{0 . 9 5} \pm 0.02$ & $\mathbf{1 . 0 8} \pm 0.03$ & $\mathbf{1 . 1 3} \pm 0.04$ & $\mathrm{n}=64$ \\
mTIRAP 4 NSLP65_wt & $\mathbf{0 . 9 3} \pm 0.01$ & $\mathbf{1 . 0 6} \pm 0.03$ & $\mathbf{1 . 1 5} \pm 0.03$ & $\mathrm{n}=66$ \\
mTIRAP $\triangle$ NSLP65_M23 & $\mathbf{0 . 9 3} \pm 0.02$ & $\mathbf{0 . 9 9} \pm 0.03$ & $\mathbf{1 . 0 8} \pm 0.04$ & $\mathrm{n}=55$ \\
\hline
\end{tabular}

Relative membrane localisation (M) of indicated TIRAP chimeras expressed in s/p65 $5^{\%}$ DT40 B cells. $M$ reflects the mean fluorescence intensity at the plasma membrane normalised to the fluorescence intensity in the cytosol in either unstimulated ( $\left.\mathrm{M}_{\text {rest. }}\right)$ or $1 \mathrm{~min}$ BCR-stimulated DT40 B cells $\left(\mathrm{M}_{\text {stim. }}\right)$. The fold-change equals the mean of the ratios of $M_{\text {stim. }}$ and $M_{\text {rest. }}$ calculated for each cell. Quantification $( \pm S D)$ of replicates of one independent experiment is shown. See section 2.2.4 for details.

In conclusion the TIRAP $\mathrm{PIP}_{2}$ binding domain could functionally replace the $\mathrm{N}$-terminus of SLP65 (Hermann 2009), but it was not functional when fused to the R-to-A variant of SLP65. Although this is an interesting finding this setup does not allow drawing conclusions regarding the question whether membrane targeting of SLP65 can compensate for CIN85/CD2AP binding to SLP65. 


\subsubsection{Targeting of SLP65_M23 to the BCR restores its function in BCR-induced Ca ${ }^{2+}$ mobilisation}

To explore whether the interaction of CIN85/CD2AP and SLP65 can be bypassed by targeting SLP65 directly to the BCR, I designed chimeric proteins depicted in Figure 3.23A. These fusion proteins contain the tandemly arranged $\mathrm{SH} 2(\mathrm{tSH} 2)$ domains of Syk instead of the first 48 amino acids of the SLP65 N-terminus (tSH2 $N$ NSLP65_wt or tSH2 2 NSLP65_M23). The Syk tSH2 domains bind to doubly phosphorylated ITAMs in the Iga/ $\beta$ hetero-dimer of the BCR (Wienands et al. 1995).

s/p65 \% DT40 B cells were transfected with fluorescently tagged constructs coding for either slp65_wt, slp65_m23 or the respective chimeras. Figure 3.23B shows that both fusion proteins translocate to the plasma membrane in response to BCR cross-linking as analysed by live cell microscopy. Quantification of relative membrane localisation is listed in table 3.5. Furthermore, I validated that the addition of the Syk tSH2 domains did not change the interaction or loss of interaction to CIN85 or CD2AP (figure 3.23C, lane 1 and 2, respectively).

Table 3.5: Quantification of the plasma membrane localisation of the tSH2_SLP65 chimeras

\begin{tabular}{ccccc}
\hline DT40 B cell specimen & $\mathbf{M}_{\text {rest. }}$ & $\mathbf{M}_{\text {stim. }}$ & fold-change & number of cells \\
\hline tSH2 $\Delta$ NSLP65_wt & $\mathbf{1 . 0 6} \pm 0.03$ & $\mathbf{1 . 4 0} \pm 0.15$ & $\mathbf{1 . 3 2} \pm 0.11$ & $\mathrm{n}=93$ \\
tSH2 $\Delta$ NSLP65_M23 & $\mathbf{0 . 9 7} \pm 0.02$ & $\mathbf{1 . 3 6} \pm 0.07$ & $\mathbf{1 . 4 0} \pm 0.05$ & $\mathrm{n}=80$ \\
\hline
\end{tabular}

Relative membrane localisation (M) of tSH2 $\Delta$ NSLP65_wt or tSH2 2 NSLP65_M23 expressed in s/p65 ${ }^{\%}$ DT40 B cells. $M$ reflects the mean fluorescence intensity at the plasma membrane normalised to the fluorescence intensity in the cytosol in either unstimulated $\left(M_{\text {rest. }}\right)$ or 1 min BCR-stimulated DT40 B cells $\left(M_{\text {stim. }}\right)$. The fold-change equals the mean of the ratios of $M_{\text {stim. }}$ and $M_{\text {rest. }}$ calculated for each cell. Quantification $( \pm S D)$ of replicates of one independent experiment is shown. See section 2.2.4 for details. 
A)

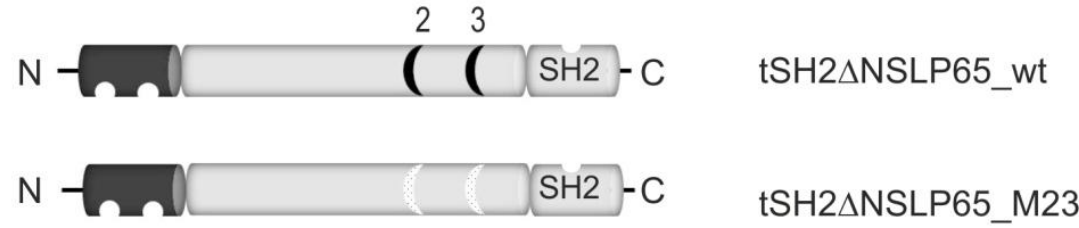

B)

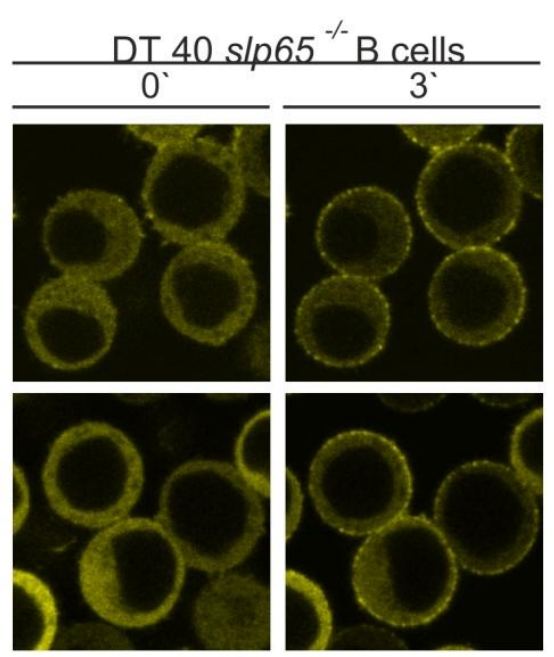

C)

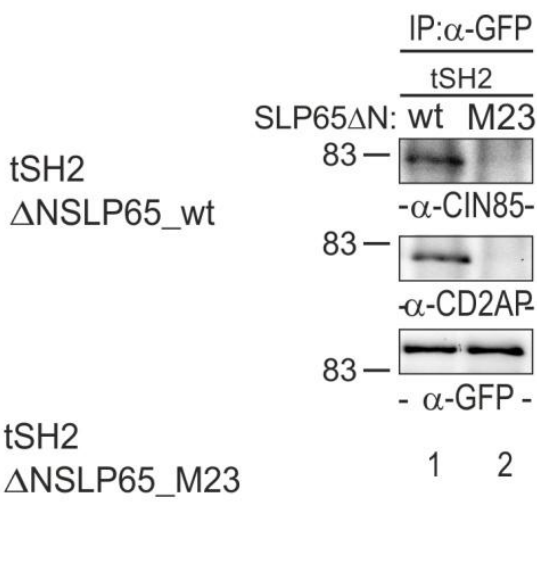

D)

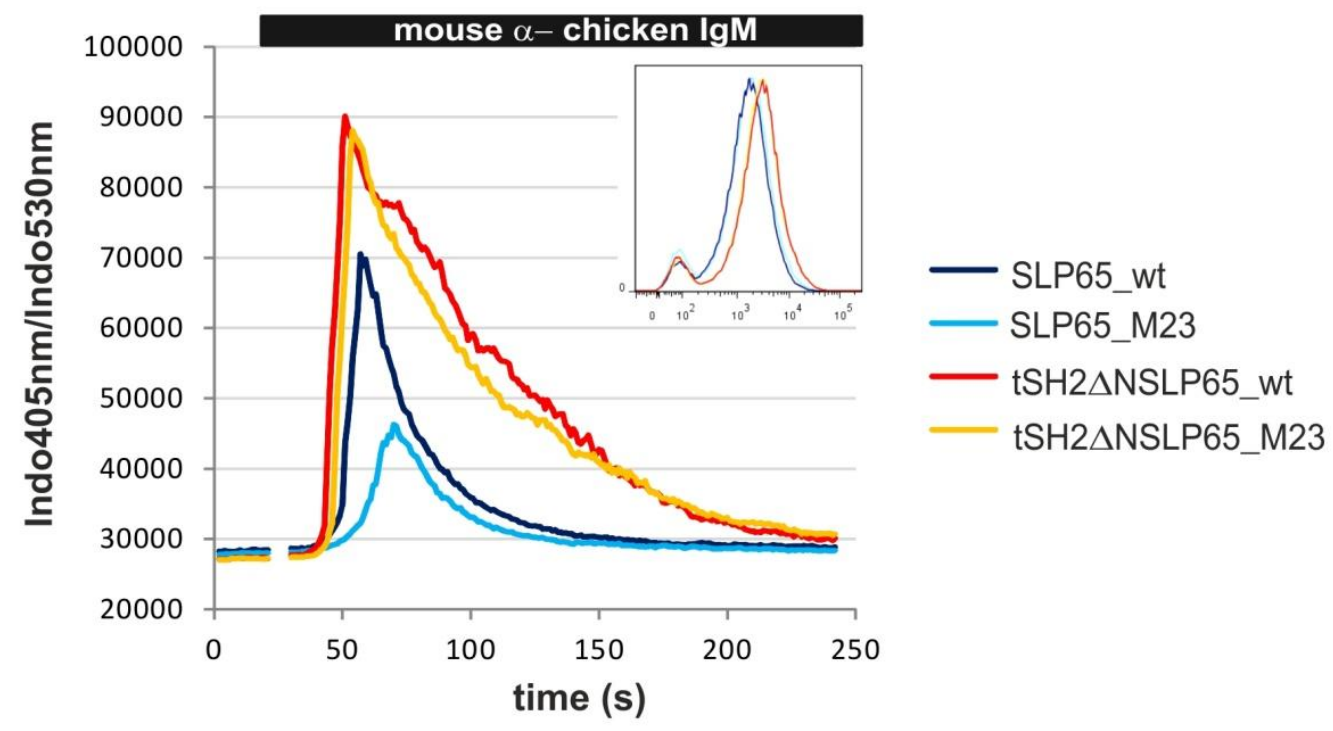

Figure 3.23: Targeting of SLP65_M23 to the BCR restores its function in $\mathrm{Ca}^{2+}$ mobilisation after BCR cross-linking.

A) Schematic representation of SLP65 chimeras in which its $\mathrm{N}$ - terminus is replaced by the tandem $\mathrm{SH} 2$ domains ( $\mathrm{tSH} 2)$ of Syk. The two remaining atypical proline-arginine motifs were left intact (top, tSH2 2 NSLP65_wt) or destroyed by an R-to-A amino acid exchange (bottom, tSH2 $\Delta$ NSLP65_M23). (continued on next page) 
(legend figure 3.23 continued) B and C) s/p65 ${ }^{-1}$ DT40 B cells were retrovirally transduced to express the chimeras described in $A$ ) and analysed for membrane recruitment by confocal laser scanning microscopy before and after BCR-stimulation ( $B$, left and right images, respectively).or $C$ ) subjected to anti-GFP immunoprecipitation followed by immunoblotting with antibodies against CIN85, CD2AP or SLP65 (from top to bottom). D) s/p65 $\%$ DT40 B cells expressing wild-type (SLP65_wt), the CIN85/CD2AP binding deficient variant (SLP65_M23) or the corresponding Syk tandem SH2 chimeras (tSH2 2 NSLP65_wt and tSH2 2 NSLP65_M23) of SLP65 were analysed for BCR induced $\mathrm{Ca}^{2+}$ mobilisation by flow cytometry using $1 \mu \mathrm{g} / \mathrm{ml}$ M4. D) inlay Flow cytometric analysis of Citrine expression of cells analysed. Apparent molecular weights of protein standards are indicated on the left in $\mathrm{kDa}$.

Next, their BCR-induced $\mathrm{Ca}^{2+}$ mobilisation profiles were investigated. Disruption of binding of SLP65 to CIN85/CD2AP is reflected by a strong inability of SLP65_M23 to mobilise Ca ${ }^{2+}$ after BCR cross-linking compared to SLP65_wt expressing B cells (figure 3.23D, compare dark and light blue lines). In contrast, once SLP65 is targeted to the BCR by fusion to the Syk tSH2 domains the $\mathrm{Ca}^{2+}$ profiles in slp65 cells expressing either tSH2 $5^{\%}$ NLP65_wt or tSH2 2 NSLP65_M23 were comparable. Hence, when SLP65 was targeted to the BCR by the Syk tSH2 domains, it did no longer depend on the interaction with CIN85 or CD2AP for proper $\mathrm{Ca}^{2+}$ mobilisation in BCR-activated cells. This indicates that the $\mathrm{Ca}^{2+}$ signalling defect in BCR-activated B cells results from dislocation of SLP65 molecules. 


\section{Discussion}

One major aim of $B$ cell biology is to scrutinise the early phase of BCR signal transduction that is characterised by the translocation of signalosomes to BCR-enriched areas in the plasma membrane. In my PhD thesis I examined the contribution of the adaptors CD2AP and CIN85 on BCR signal transduction, which previously have been found in a preformed complex with SLP65 by our group. The herein documented results contribute to the understanding of early BCR signal transduction with respect to BCR-proximal signalosomes and their importance in generating $\mathrm{Ca}^{2+}$ responses.

The main findings of this thesis are:

1) CIN85 is a positive regulator in the onset of $\mathrm{BCR}$-induced $\mathrm{Ca}^{2+}$ mobilisation by enabling $\mathrm{BCR}$-activated $\mathrm{B}$ cells to respond with a rapid and strong $\mathrm{Ca}^{2+}$ profile. In this respect CD2AP can exert a partially compensatory function, as soon as cin85 expression is reduced. The coiled coil and the SH3 domains of CIN85 are necessary for mounting an appropriate $\mathrm{Ca}^{2+}$ response after BCR cross-linking.

2) CIN85 and CD2AP are recruited to the plasma membrane upon stimulation of the BCR, but use different anchoring modes. CIN85 membrane recruitment depends on its SH3 domains, but not the cytoskeleton. In contrast, CD2AP membrane localisation is less dependent on its SH3 domains, but sensitive to actin inhibitors. However, both molecules require their coiled coil domain for tethering to the plasma membrane.

3) CIN85 colocalises with BCR-containing microclusters upon BCR engagement. This colocalisation is evident throughout the whole response of the $B$ cell to stimulating lipid bilayers. This includes early microcluster as well as later formation of the central BCR cluster. Unlike CIN85, only very few CD2AP molecules were found to colocalises with the microclusters. The BCR signal that is obligatory for CIN85 plasma membrane translocation and presumably BCR colocalisation is transduced by Lyn, but not Syk. A role of CIN85 in BCR internalisation was not observed.

4) The preformation of CIN85/CD2AP with SLP65 is based on an interaction of the SH3 domains of CIN85/CD2AP and the second and third proline-arginine motif in SLP65. CIN85 and CD2AP bind SLP65 independent of each other and can hetero-oligomerise via their coiled coil domains. Importantly, the disruption of the preformed complex comprising SLP65 and CIN85/CD2AP is bypassed by providing SLP65 with direct access to the BCR. 


\subsection{CIN85, CD2AP and SLP65 - complex BCR signalling}

\subsubsection{The preformed complex of CIN85 and SLP65 in BCR-induced $\mathrm{Ca}^{2+}$ mobilisation} In this thesis the role of CD2AP and CIN85 in the BCR-triggered $\mathrm{Ca}^{2+}$ mobilisation, was analysed in a cd2ap $\%$ DT40 B cell line and a genetic reconstitution experiment after RNAi. With this setup, I was able to specifically link the shRNA-evoked effect to the expression of CIN85 or CD2AP. I could demonstrate that already the knock-down of cin85 expression had a negative impact on $\mathrm{Ca}^{2+}$ flux, while complete CD2AP-deficiency had none. Thus, I assume that CIN85 has the dominant role in the BCR-induced $\mathrm{Ca}^{2+}$ pathway. However, in combination with CD2AP-deficiency interference with cin85 expression resulted in an even more delayed and decreased BCR-induced $\mathrm{Ca}^{2+}$ flux, which implies that CD2AP can partially compensate for diminished CIN85 abundance. This is further supported by the notion that both CIN85 and CD2AP are able to restore $\mathrm{Ca}^{2+}$ mobilisation in the "absence" of each other making a concerted action of CIN85 and CD2AP rather unlikely. These data are in direct accordance with the initial observation that prevention of SLP65 binding to CIN85/CD2AP resulted in a strong defect in $\mathrm{Ca}^{2+}$ mobilisation (Oellerich et al. 2011). Taken together this provides evidence that a preformed CIN85 and SLP65 module operates in the rapid initiation and progression of $\mathrm{BCR}$-induced $\mathrm{Ca}^{2+}$ flux, as was predicted, but not identified earlier in our group (Wienands et al. 1996). The contribution of CIN85 to SLP65 function will be discussed in section 4.2 .

This positive regulatory effects of CIN85 in BCR signalling is corroborated by the phenotype of mice with a $B$ cell-specific deletion of the cin85 gene (Kometani et al. 2011). The cin85 mice exhibited reduced numbers of B-1 B cells and failed to elicit a T cell-independent type II immune response. Common to both, maintenance of the B-1 B cell population and the $T$ cellindependent activation of $B$ cells is an almost exclusive stimulation of the BCR i.e. by natural/self antigen or highly repetitive, multivalent antigen, respectively (Hayakawa et al. 1999; Fagarasan and Honjo 2000; Vos et al. 2000). This, together with the colocalisation of CIN85 to BCR-containing microclusters, supports a function of CIN85 specifically downstream of the BCR. The effect of CIN85 in the herein analysed $\mathrm{Ca}^{2+}$ mobilisation was more pronounced after low BCR stimulation and rather negligible after BCR-stimulation with higher density of anti-chicken IgM. Likewise, the mitogenic response of $\operatorname{cin} 85^{\%}$ murine $\mathrm{B}$ cells to mitogenic BCR stimuli showed a titratable sensitivity in in vitro experiments, but nonetheless CIN85 was indispensable for in vivo B cell proliferation (Kometani et al. 2011). Thus, the experimental use of low amounts of BCR cross-linker, might be closer to in vivo $\mathrm{B}$ cell activation than maximal BCR stimulation.

The $\operatorname{cin} 85^{-1} \mathrm{~B}$ cells displayed a defect in the NFKB pathway, but neither in overall tyrosine phosphorylation of BCR proximal downstream effector molecules nor in BCR-induced $\mathrm{Ca}^{2+}$ 
mobilisation (Kometani et al. 2011). The failure to observe a function of CIN85 in this respect is likely due to the compensation by CD2AP as demonstrated herein for the DT40 system.

Studies in T cells demonstrated a positive role of CD2AP in pre TCR and TCR signalling (Dustin et al. 1998; Lee et al. 2003; Navarro et al. 2007), which would be similar to the compensatory, positive effects of CD2AP in BCR-induced $\mathrm{Ca}^{2+}$ signalling in B cells.

Contrary to the publication of Kometani and data from this thesis, a recent publication investigating the function of CIN85 in human B cells postulate an inhibitory role of CIN85 (Niiro et al. 2012). Overexpression of CIN85 in the BJAB B cell line resulted in decreased phosphorylation of Syk, SLP65 or PLCY2 (among others) and BCR-induced $\mathrm{Ca}^{2+}$ mobilisation, while knock-down of cin85 mRNA expression led to increased protein phosphorylation and $\mathrm{Ca}^{2+}$ flux. The authors hypothesise that CIN85 forms an inhibitory module together with $\mathrm{Cbl}$. The discrepancy between the cin85 knock-down data of Niiro et al. and the data from this thesis might be easily explained by the different nature of the $B$ cell types analysed.

The $\mathrm{Ca}^{2+}$ defect observed in cd2ap ${ }^{-1}$ DT40 B cells together with reduced cin85 expression was not as pronounced as the defect of the SLP65 R-to-A variant that does no longer bind CIN85/CD2AP. This is likely due to the use of RNAi instead of a CIN85-deficient DT40 B cell line, in which residual CIN85 expression might dilute the effects. Noteworthy, the observed differences might depend on the species from which SLP65 was derived. For $\mathrm{Ca}^{2+}$ mobilisation analysis of the SLP65 R-to-A variants, human slp65 cDNA was used. In the cd2ap ${ }^{*}$ DT40 B cells a dependence on CD2AP/CIN85 could only be analysed for endogenous chicken SLP65. Indeed, data from a diploma thesis carefully analysing the SLP65 species differences suggests that chicken SLP65 is less dependent on CIN85/CD2AP interaction than human SLP65 (K.Henzel, diploma thesis). A function of Grb2 in SLP65mediated $\mathrm{Ca}^{2+}$ mobilisation cannot be excluded, because binding of Grb2 to SLP65 was reduced upon changes in the proline-arginine motifs in SLP65 (Oellerich et al. 2011). However, Grb2 is a negative regulator in BCR-induced $\mathrm{Ca}^{2+}$ signalling of immature $\mathrm{B}$ cells and not known to positively influence SLP65 phosphorylation in DT40 B cells (Stork et al. 2004). This, and the specificity of the PxxxPR motifs for CIN85 and CD2AP SH3 domains, argues in favour of CIN85 as the important binding partners for the SLP65 proline-arginine motifs in BCR-induced $\mathrm{Ca}^{2+}$ signalling.

\subsubsection{CIN85 - a true scaffold for protein complexes}

Interestingly, only upon tightly defined cin85 expression, DT40 B cells showed a BCRinduced $\mathrm{Ca}^{2+}$ profile comparable to control cells. Diminished cin85 expression (as in the knock-down situation) or strong overexpression of cin85 resulted in a decrease of BCRinduced $\mathrm{Ca}^{2+}$ flux. Non-physiological abundance of CIN85 might result in changes in the 
formation of naturally occurring protein complexes: Instead of one CIN85 molecule (CIN85) forming one functional, e.g. trimeric complex $(\mathrm{CIN85+B+C})$, two CIN85 molecules are used forming two non-functional dimers $(\mathrm{CIN85+B}, \mathrm{CIN85+C})$. This is known as combinatorial inhibition (Levchenko et al. 2000). For the yeast mating pathway Chapman et al. showed that signal throughput in yeast cells did depend on the abundance of the scaffold Sterile 5 (Ste5) in a biphasic manner. Below and above an optimal concentration of Ste5, signalling was altered (Chapman and Asthagiri 2009). My data suggests that CIN85 displays a biphasic character as well. Since cd2ap expression was not as high as cin85 expression a biphasic character could not be detected for CD2AP technically. Importantly, the sensitivity to imbalanced expression suggests that CIN85 serves as a backbone for multimolecular complex formation in BCR-induced $\mathrm{Ca}^{2+}$ mobilisation. This complex involves the $\mathrm{SH} 3$ domains since overexpression of a SH3 domain truncation variant of CIN85 is dominantnegative in $\mathrm{Ca}^{2+}$ signalling. In contrast, the coiled coil truncation variant of CIN85 displays no biphasic character (data not shown). Hence, CIN85 might scaffold e.g. SLP65 and yet unknown proteins via its $\mathrm{SH} 3$ domains and proline-rich motifs in cis. Interestingly, in silico analysis (http://scansite.mit.edu/motifscan_seq.phtml) of possible interaction partners of CIN85 and CD2AP revealed hits for PLCY1 and Itk, respectively. It is thus very tempting to speculate that CIN85 or CD2AP could stabilise the $\mathrm{Ca}^{2+}$ initiation complex comprising SLP65, Btk and PLCY2. The elucidation of CIN85-based complexes is currently addressed with a mass spectrometric approach by our group.

\subsubsection{CIN85 and CD2AP: twins or siblings?}

In my experimental system partial redundancy of CIN85 and CD2AP was demonstrated by the enhanced defect in $\mathrm{BCR}$-induced $\mathrm{Ca}^{2+}$ mobilisation after interference with cin85 expression in combination with CD2AP-deficiency. Studies with CD2AP- or CIN85-deficient cells dampened the originally assigned function of CD2AP in the immunological synapse and for CIN85 in receptor internalisation (Lee et al. 2003; Shimokawa et al. 2010), which might have been less pronounced due to functional compensation by CIN85 or CD2AP, respectively. Functional redundancy of CIN85 and CD2AP is very likely due to the high similarity of the proteins. Indeed phylogenetically they are paralogues descending from a gene duplication event after the split of vertebrates from invertebrates (Tossidou et al. 2011). Looking on the primary sequence which shares $41 \%$ amino acid identity and the same overall domain architecture CIN85 and CD2AP SH3 domains share more similarities among themselves than with any other SH3 domain (Dikic 2002). A likely explanation for the redundancy is that CIN85 and CD2AP have similar binding qualities; e.g. both bind CD2 
(Dustin et al. 1998; Tibaldi and Reinherz 2003), Cbl (Take et al. 2000; Kirsch et al. 2001) and SLP65.

The association of the preformed complex comprising CIN85 and/or CD2AP (CIN85/CD2AP) and SLP65 is based on the SH3 domains of CIN85/CD2AP and the atypical proline-arginine motifs in SLP65 (Kowanetz et al. 2003; Kurakin et al. 2003) and could be confirmed in this study. Immuno purifications of full length CD2AP or CIN85 have not been conducted herein, but would further proof the interaction with SLP65. A stimulation-dependent alteration of the interaction could not be observed in DT40, primary mouse and human Ramos B cells by either mass spectrometry or biochemistry (Oellerich et al. 2011). However Niiro et al. claim to observe an increase in SLP65/CIN85 interaction after BCR stimulation in the human BJAB B cell line, nonetheless there is substantial association already in unstimulated B cells (Niiro et al. 2012). Hence the term preformed is appropriate to describe the stimulation-independent association of CIN85/CD2AP and SLP65. This is in accordance with the binding of SH3 domains and their ligands, which - unlike $\mathrm{SH} 2$ domains do not rely on posttranslational modifications (for review see (Mayer 2001)).

SLP65 contains three atypical proline-arginine motifs thus being a selected binding partner of the CIN85/CD2AP family rather than other SH3 domain containing proteins. Indeed, mass spectrometry showed that upon destruction of these motifs in a SLP65 peptide CIN85 and CD2AP got specifically lost, albeit there is also diminished association of the subunits of $F$ actin capping protein (CapZ) and Grb2 with SLP65 (Oellerich et al. 2011). CapZ is an interaction partner of CIN85/CD2AP and harbours no obvious binding motif for a direct SLP65 interaction (Hutchings et al. 2003). Thus, CapZ probably gets lost from SLP65 due to interaction with CIN85 or CD2AP. Although there are publications describing an interaction of CIN85 with Grb2 (Kirsch et al. 1999; Borinstein et al. 2000; Watanabe et al. 2000), mass spectrometry performed in our group did not reveal CIN85 or CD2AP as part of the Grb2 interactome in murine Bal17 B cells (Neumann et al. 2009). It would be worth to study whether Grb2 and CIN85/CD2AP can interact in the DT40 B cell system.

Destruction of the second (PSPLPR) or the third proline-arginine motif (PIPLPR) in SLP65 resulted in abolished or decreased binding of CIN85/CD2AP, respectively. This is in line with data by Kowanetz et al. but additionally showed that CD2AP had the very same binding characteristics to SLP65 as CIN85 had (Kowanetz et al. 2004). Thus, I propose a binding mechanism in which recognition of the second SLP65 proline-arginine motif by one SH3 domain of CIN85/CD2AP is necessary, but to be sufficient a second SH3 domain in CIN85/CD2AP must bind the third proline-arginine motif in SLP65 (see figure 4.1A). The differential importance of the proline-arginine motifs for CIN85/CD2AP binding is in accordance with the BCR-induced $\mathrm{Ca}^{2+}$ mobilisation profiles of the single SLP65 R-to-A variants (Oellerich et al. 2011). Destruction of the second proline-arginine motif in SLP65 led 
to more severe impairment in $\mathrm{BCR}$-induced $\mathrm{Ca}^{2+}$ flux than destruction of the third motif, while a combination of both displayed the strongest defect in this assay. Alteration of the first proline-arginine motif (PPSVPR) of SLP65 neither impaired the interaction with CIN85/CD2AP nor BCR-induced $\mathrm{Ca}^{2+}$ signalling. It can be discriminated from the two other proline-arginine motifs (PxPxPR) by lacking the central proline (PxxxPR). Previous analysis of dynamic phosphorylation of chicken SLP65 identified two conserved serine residues in the first or second proline-arginine motif as BCR-induced or down-regulated phosphorylation sites, respectively (Oellerich et al. 2009). Since the first proline-arginine motif is possibly not bound by CIN85/CD2AP it would be accessible for a kinase. A phosphorylated serine residue in the second proline-arginine motif would possibly not be tolerated by CIN85/CD2AP SH3 domains. An involvement of serine phosphorylation in CIN85/CD2AP and SLP65 interaction needs further experimentation.

Watanabe et al. showed that the three SH3 domains of CIN85 together purified more SLP65 than the single SH3 domains (Watanabe et al. 2000). This could not be confirmed by our group. Affinity purifications with the three SH3 domains of either CIN85 or CD2AP did not purify more SLP65 than the individual SH3 domains (J. Gerstmaier, diploma thesis). The respective contribution of the individual SH3 domains of CIN85/CD2AP for SLP65 binding would be worth to elucidate by mutational analyses. It would be interesting to further analyse the binding mechanisms of SLP65 with CD2AP and CIN85 especially with respect to dissociation constants. A clue to differences between CIN85 vs. CD2AP function with respect to SLP65 might lie in their affinity for SLP65.

Still, the stoichiometry of the preformed complex comprising SLP65 and CIN85/CD2AP needs further elucidation. SLP65 has been found in a preformed $180 \mathrm{kDa}$ complex by 2 dimensional blue native polyacrylamid gel electrophoresis in unstimulated K46 B cells (Swamy et al. 2006). Possible interaction partners of SLP65 were not elucidated in this study, thus it would be interesting to see whether CIN85 and/or CD2AP can be found preformed with SLP65 in this setup.

Due to the same binding behaviour of CIN85 and CD2AP to SLP65, it is difficult to envision how these two molecules would bind to one SLP65 molecule at the same time forming a trimeric SLP65/CIN85/CD2AP complex. Moreover an interaction of one SH3 domain of CIN85 and one SH3 domain of CD2AP with the second and third proline-arginine motif, respectively, is unlikely since the SLP65_M2 variant (destruction of the SLP65 second proline-arginine motif) did not copurify any CD2AP or CIN85 with its intact third prolinearginine motif. The independence of each other for binding SLP65 allows individual nontrimeric complexes. In addition the coiled coil domains of CIN85 and CD2AP can homo/hetero-oligomerise opening the possibility of an at least tetrameric complex with two SLP65 molecules (see figure 4.1B). A functional importance for SLP65 clustering due to 
homo-oligomerisation of CIN85 or CD2AP molecules needs further investigation. A need for hetero-oligomerisation of CIN85 and CD2AP is rather unlikely, because, instead of showing a concerted action in BCR-induced $\mathrm{Ca}^{2+}$ mobilisation, CIN85 and CD2AP are partially redundant.

The biochemical analysis provided insight into the molecular details of the formation of CD2AP/SLP65 and CIN85/SLP65 protein complexes, but is less suitable to reveal functional differences between CIN85 and CD2AP. Investigations with living cells, like in $\mathrm{Ca}^{2+}$ mobilisation or microscopical assays (see section 4.2.1), are more powerful in this respect.

A)

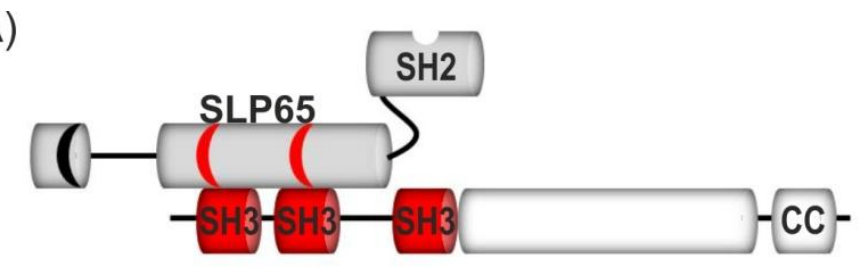

CIN85/CD2AP

B)

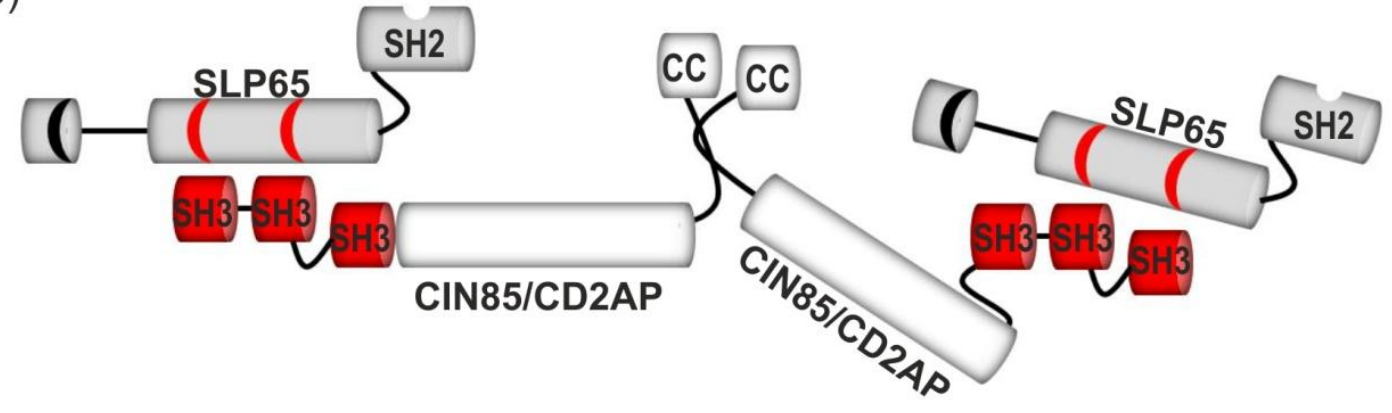

Figure 4.1: Model for the interaction of CIN85 or CD2AP with SLP65.

Two of the three SH3 domains of CIN85/CD2AP (red) interact with the second and third atypical proline-arginine motif in SLP65 ((). This results in formation of either CIN85/SLP65 or CD2AP/SLP65 dimers (A) or oligomers (B). Homo-oligomerisation of CIN85 or CD2AP involves the coiled coil (CC) domains and results in clustering of SLP65 molecules. Even higher-order oligomers can be formed (not shown).

Post-translational modifications could contribute to (differential) function of CIN85 and CD2AP; e.g. CIN85 and CD2AP are known to be ubiquitinated (Haglund et al. 2002);(Verdier et al. 2002). CD2AP contains 5 tyrosine phosphorylation motifs, while in CIN85 exists only one, but tyrosine phosphorylation of CD2AP and CIN85 could not be detected in DT40 B cells (Tibaldi and Reinherz 2003). Whole phospho-proteome mass spectrometric approaches already elucidated threonine- and serine phosphorylation sites on CD2AP or CIN85 
(http://www.phosphosite.org). Former analysis from our group revealed the importance of serine phosphorylation in SLP65 and Syk (Oellerich et al. 2009; Bohnenberger et al. 2011). Hence, exploration of serine/threonine phosphorylation in CIN85 and CD2AP could reveal functional aspects of the scaffolds in BCR signalling.

Table 4.1 Structural and functional features of CIN85 and CD2AP

\begin{tabular}{|c|c|c|c|c|}
\hline feature & CIN85 & CD2AP & similarity & ref. \\
\hline \multicolumn{5}{|l|}{ structure: } \\
\hline $3 \mathrm{~N}$-terminal SH3 domains & yes & yes & & \\
\hline \multirow[t]{2}{*}{$1^{\text {st }} \mathrm{SH} 3$ domain (aa) } & & & $60 \%$ & (Tibaldi and \\
\hline & & & & Reinherz 2003) \\
\hline \multirow[t]{2}{*}{$2^{\text {nd }} \mathrm{SH} 3$ domain (aa) } & & & $72 \%$ & (Tibaldi and \\
\hline & & & & Reinherz 2003) \\
\hline \multirow[t]{2}{*}{$3^{\text {rd }} \mathrm{SH} 3$ domain (aa) } & & & $60 \%$ & (Tibaldi and \\
\hline & & & & Reinherz 2003) \\
\hline coiled coil domain & yes & yes & $37 \%$ & \\
\hline basic pl & 8.68 & 8.25 & & expasy.org \\
\hline proline-rich motifs (number) & 4 & 3 & & \\
\hline \multirow[t]{2}{*}{$1^{\text {st }}$ proline-rich motif } & & & $100 \%$ & (Tibaldi and \\
\hline & & & & Reinherz 2003) \\
\hline \multirow[t]{2}{*}{$3^{\text {rd }}$ proline-rich motif } & & & $75 \%$ & (Tibaldi and \\
\hline & & & & Reinherz 2003) \\
\hline \multirow[t]{2}{*}{$4^{\text {th }}$ proline-rich motif } & & & $67 \%$ & (Tibaldi and \\
\hline & & & & Reinherz 2003) \\
\hline \multirow[t]{2}{*}{ PEST sequence (number) } & 1 & 1 (less) & & (Tibaldi and \\
\hline & & & & Reinherz 2003) \\
\hline \multirow[t]{2}{*}{ actin binding motifs } & 0 & 4 & & (Tibaldi and \\
\hline & & & & Reinherz 2003) \\
\hline \multirow[t]{2}{*}{ isoforms } & yes & no & & (Buchman et al. \\
\hline & & & & 2002) \\
\hline \multirow[t]{2}{*}{ intramolecular folding } & yes & no & & (Kowanetz et al. \\
\hline & & & & 2003) \\
\hline \multicolumn{5}{|l|}{ regulation (potential): } \\
\hline proteolysis & yes & no & & phosphosite.org \\
\hline tyrosine phosphorylation & 1 & 4 & & (Tibaldi and \\
\hline (number of sites) & & & & Reinherz 2003) \\
\hline threonine phosphorylation & yes & yes & & phosphosite.org \\
\hline
\end{tabular}




\begin{tabular}{|c|c|c|c|}
\hline serine phosphorylation & yes & yes & phosphosite.org \\
\hline ubiquitination & yes & yes & $\begin{array}{r}\text { (Haglund et al. } \\
\text { 2002) }\end{array}$ \\
\hline SUMOylation & yes & not known & $\begin{array}{r}\text { (Tossidou et al. } \\
\text { 2011) }\end{array}$ \\
\hline \multicolumn{4}{|l|}{ BCR signalling (this thesis): } \\
\hline $\begin{array}{l}\text { plasma membrane recruitment, } \\
\text { depends on }\end{array}$ & yes & yes & \\
\hline SH3 domains & yes & no & \\
\hline coiled coil domain & yes & yes & \\
\hline actin cytoskeleton & no & yes & \\
\hline $\begin{array}{l}\text { positive regulatory role in the } \\
\text { onset of } \mathrm{Ca}^{2+} \text { flux }\end{array}$ & yes & $\begin{array}{l}\text { com- } \\
\text { pensatory }\end{array}$ & \\
\hline BCR colocalisation & strong & weak & \\
\hline SLP65 binding & yes & yes & \\
\hline
\end{tabular}

\subsection{SLP65's third tooth for biting the membrane}

A bidentate membrane anchoring mode for SLP65 with the $\mathrm{N}$-terminus providing general membrane localisation, while the $\mathrm{SH} 2$ domain provides $\mathrm{BCR}$ proximal localisation was discussed before by our group (Abudula et al. 2007). A cooperation of the two domains was demonstrated to stabilise SLP65 at the plasma membrane (Hermann 2009). The interaction of CIN85/CD2AP with SLP65 could add a third mechanism to the plasma membrane localisation of SLP65.

\subsubsection{Sub cellular localisation of CIN85 and CD2AP}

The precise sub cellular localisation of B cell effector proteins is crucial for BCR signal transduction. Microscopy is a powerful method for the analysis of membranous vs. cytosolic location of fluorescent proteins, since BCR-induced translocations can be followed in living, intact cells. The microscopic analysis of CD2AP and CIN85 revealed that recruitment to the plasma membrane required their coiled coil domain. Coiled coil domains are present in many proteins associated with the cytoskeleton. CD2AP and CIN85 were shown to colocalise with actin-rich membrane ruffles (Kirsch et al. 1999) or actin (Schmidt et al. 2003) and to bundle F-actin dependent on their coiled coil domain and proline-rich motifs (Gaidos et al. 2007). This could explain why the coiled coil truncation variant of CD2AP did not localise to the 
plasma membrane, and the sensitivity of CD2AP membrane localisation to the actin inhibitor Latrunculin B. Thus, it can be assumed that CD2AP plasma membrane localisation depends on an integral cytoskeleton in unstimulated as well as stimulated $B$ cells. This might involve either a direct interaction with F-actin via CD2AP`s four actin binding motifs (which are lacking in CIN85) (Kirsch et al. 1999) or might be indirect via cytoskeleton-associated binding partners of CD2AP, like CapZ, $\mathrm{p}^{130}$ Cas or cortactin via the proline-rich motifs of CD2AP (Kirsch et al. 1999; Hutchings et al. 2003; Lynch et al. 2003). Both mechanisms are in accordance with the ability of the C-terminal half of CD2AP to sufficiently translocate to the plasma membrane (see figure 4.2, right).
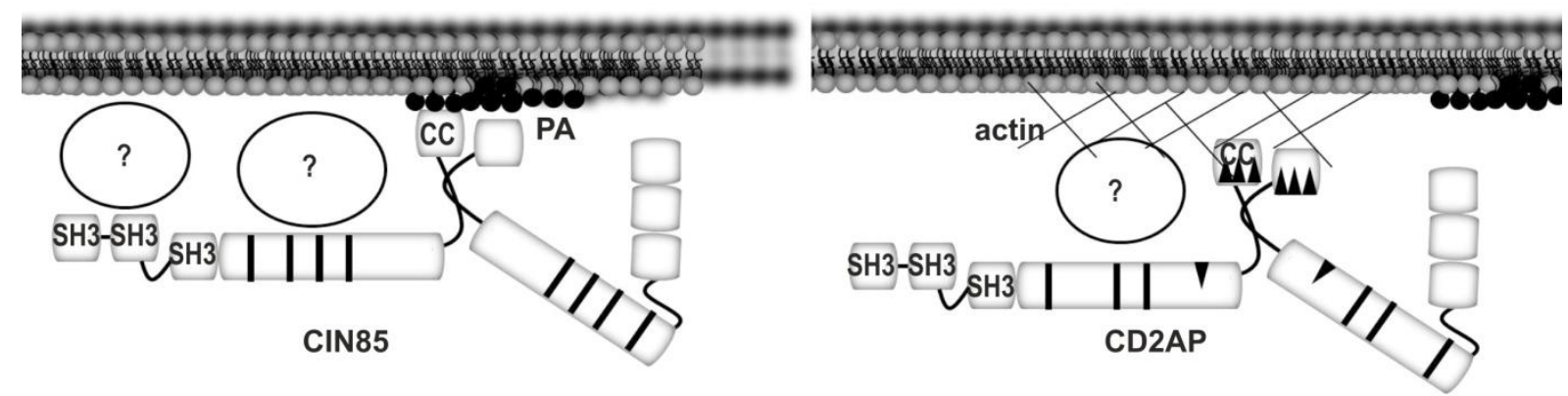

\section{Figure 4.2: Model for the mechanisms of CIN85 and CD2AP plasma membrane recruitment}

For anchoring to the plasma membrane CIN85 and CD2AP use different modes: CIN85 requires its coiled coil domain either for binding phosphatidic acid (PA, black dots), homo-oligomerisation or interaction with yet unknown proteins. Moreover the SH3 domains or the proline-rich motifs of CIN85 mediate binding to yet unknown ligands. In contrast, CD2AP is either directly anchored to the actin cytoskeleton via its actin-binding motifs (black triangles) or interacts with cytoskeletal adaptor proteins via its coiled coil domain or proline-rich motifs. The $\mathrm{SH} 3$ domains are not involved in anchoring CD2AP to the plasma membrane.

Unlike CD2AP, CIN85 membrane localisation required the coiled coil as well as the SH3 domains and was rather insensitive to Latrunculin B. The SH3 domains mediate proteinprotein interactions and possible B cell specific interaction partners of CIN85 might include those identified by a mass spectrometric approach (Buchse et al. 2011). The dependence of CIN85 membrane recruitment on interaction partners would be worth to study and will be discussed in section 4.2.2. The coiled coil domain of CIN85 was shown to bind to phosphatidic acid likely via electrostatic interactions (Zhang et al. 2009). Phosphatidic acid comprises $1-4 \%$ of all membrane lipids and the major pathway of its generation is hydrolysis of phosphatidylcholine by activated Phospholipase D (PLD) (for review see (Selvy et al.; Stace and Ktistakis 2006)). Given that the signal for CIN85 translocation is transduced by Lyn, but independent of Syk, PLD activation should be independent of Syk, too. This could not be observed in investigations of PLD activity after BCR cross-linking in the DT40 system, which revealed a dependence on Syk, Btk and PLCY2, but not Lyn (Hitomi et al. 1999). 
However, PLD activation must not necessarily precede CIN85 membrane translocation if the constant pools of phosphatidic acid in the plasma membrane are sufficient to stabilise CIN85 there. A dependence of CIN85 on phosphatidic acid in B cells would be worth to address, by either directly adding phosphatidic acid or overexpression of PLD in DT40 B cells (Zhao et al. 2007). A function of coiled coil domains in oligomerisation and localisation was shown for CARD-containing MAGUK protein 1 (CARMA1) in T cells (Tanner et al. 2007). Of course the coiled coil domain could function in homo-oligomerisation of CIN85 molecules thereby mediating CIN85 membrane translocation (see figure 4.2, left). CIN85 membrane recruitment is likely to be independent of hetero-oligomerisation with CD2AP, since membrane translocation of CIN85 occurred in cd2ap ${ }^{-/}$DT40 B cells to normal levels.

\subsubsection{CIN85 - a novel resident of BCR-containing microclusters}

High resolution TIRF microscopy performed in collaboration with the group of Dr.F. Batista demonstrated for the first time that CIN85 is part of BCR microclusters in B cells during the whole biphasic spreading and contraction response. Thereby CIN85 joins the list of other BCR signal transducers (e.g. Lyn, Syk, PLCY2) that colocalise to BCR-containing microclusters (Fleire et al. 2006; Sohn et al. 2008; Weber et al. 2008; Oellerich et al. 2011). An association of CIN85 with the BCR was also observed by two complementary approaches i.e. by confocal microscopy of Citrine-tagged CIN85 expressing DT40 B cells with differently fluorescently labelled BCR (data not shown (Kometani et al. 2011)) and coimmuno purifications of the surface BCR with CIN85. Although all of the data demonstrate an association of CIN85 and the BCR, conclusions towards CD2AP and its relation to the BCR are difficult to draw. At least, it is indicated that BCR association with CD2AP is much weaker compared to CIN85. However, different expression levels of the two proteins might have had an influence on that.

CIN85 microcluster localisation is likely not due to a direct interaction with the BCR. Although I was able to precipitate CIN85 with the BCR, this could also be due to an indirect interaction, which is further supported by the fact that the intracellular part of chicken IgM does not comprise proline-rich motifs, $\mathrm{SH} 3$ domains or coiled coils to mediate a direct interaction with CIN85. Given that the lysis condition applied was very mild whole membrane complexes might have been purified. This opens the possibility that other proteins in the microclusters were the actual interaction partner of CIN85. TIRF microscopy of CIN85 in s/p65 $\%$ DT40 B cells showed that CIN85 BCR colocalisation was independent of SLP65, thus, SLP65 is unlikely to have a membrane-anchoring function for CIN85. Grb2 localises to BCR-containing microclusters in DT40 B cells as well (Schnyder et al. 2011), but a membrane-anchoring function of Grb2 for CIN85 is rather unlikely because CIN85 could still localise to the plasma membrane in the absence of Grb2. Therefore also Downstream of kinase 3 (Dok3) and Cbl 
are unlikely candidates, because their recruitment to microclusters was shown to depend on Grb2 (Schnyder et al. 2011). However, in favour of Cbl as a membrane anchor for CIN85 speaks that in the absence of Cbl and Cbl-b SLP65 is less phosphorylated (Kitaura et al. 2007) as is SLP65 when not bound to CIN85 (Oellerich et al. 2011). Dependence of CIN85 membane recruitment on Lyn kinase activity puts Lyn itself or its substrates into the focus. CD2AP was shown to copurify with the SH3 domains of the Src kinases Fyn, Src and Yes, which points to an affinity of Lyn SH3 domains to CIN85 as well (Kirsch et al. 2001). Indeed, CIN85 has been shown to indirectly associate with tumour necrosis factor receptor 1 (TnfR1) via Src (Narita et al. 2005). However, in $l y n^{-/}$DT40 B cells CIN85 can translocate to the membrane albeit with a pronounced delay and aberrant pattern (data not shown). The B cell marker CD19 is as substrate of Lyn and a transient resident of the microsignalosomes (Depoil et al. 2008), however expression of a chicken homologue is not proven. Another, albeit only partial, substrate of Lyn is B-cell phosphoinositide 3-kinase adapter protein 1 (BCAP) (Okada et al. 2000). It is enriched in lipid rafts and would be a bona fide CIN85 interacting protein; it comprises a coiled coil domain and three atypical proline-arginine motifs. Thus, multiple proteins are candidates to be involved in CIN85 membrane localisation and need further elucidation.

CIN85 mediates ligand-dependent internalisation of several RTKs via scaffolding a CblCIN85-endophilin complex (Petrelli et al. 2002; Soubeyran et al. 2002; Szymkiewicz et al. 2002). A role of CIN85 in BCR internalisation could not be observed by me or others in either chicken DT40 B cells, human BJAB B cells and CIN85-deficient primary mouse B cells (Niiro et al.; Kometani et al. 2011; Niiro et al. 2012). Although BCR down modulation seems independent of CIN85, it requires $\mathrm{Cbl}$ proteins as demonstrated by a higher amount of surface BCR in $\mathrm{cbl}^{-/} \mathrm{cbl-b} \mathrm{b}^{-/}$primary B cells and decreased BCR internalisation in these cells compared to wt controls (Kitaura et al. 2007). Thus the CIN85-Cbl-endophilin is employed by certain receptors while others like the BCR do not depend on this internalisation complex. Analysis of internalisation of dopamine receptors in mice lacking expression of the brain specific CIN85 isoforms revealed that CIN85 facilitated complex formation of endophilin with the D2 dopamine receptor and subsequent D2 dopamine receptor internalisation, while D1 dopamine receptor internalisation was not affected (Shimokawa et al. 2010). CD2AP was shown to be involved in $T$ cell receptor degradation, but not internalisation (Lee et al. 2003). Thus, a function of CIN85/CD2AP in BCR degradation could be hypothesised in B cells as well. 


\subsubsection{CIN85 and SLP65 - a preformed and BCR-targeted module}

Several lines of evidence support the hypothesis that CIN85/CD2AP target SLP65 to the plasma membrane to allow further BCR signal transduction: CIN85 and CD2AP translocate to the plasma membrane upon BCR stimulation, thus, fulfilling a requirement for targeting SLP65 there. The domains involved in CIN85 membrane recruitment, were also needed for proper $\mathrm{Ca}^{2+}$ fluxing, which points to functional relation of these two BCR-induced processes. Importantly, our group (T.Oellerich and Dr. M. Engelke) could show that SLP65 did only efficiently translocate to the plasma membrane when harbouring the binding motifs for CD2AP/CIN85. Specificity and functionality of the atypical proline-arginine motifs and their recognition by CIN85 was additionally shown by our group by reintroducing the three prolinearginine motifs of SLP65 into its T cell paralogue SLP76 (H. Bohnenberger). SLP76 is very similar to SLP65 in sequence but can only reconstitute signalling function in s/p65 $\mathrm{B}$ cells when co-expressed with its membrane anchor LAT (Ishiai et al. 2000; Wong et al. 2000). SLP76 equipped with the binding sites for CIN85/CD2AP translocated to the plasma membrane and restored BCR-induced $\mathrm{Ca}^{2+}$ mobilisation in the absence of LAT (Oellerich et al. 2011). To dissect whether general plasma membrane localisation of SLP65 would be sufficient to circumvent CIN85/CD2AP binding to SLP65, I generated TIRAP/SLP65 chimeras. Although the N-terminus of SLP65 could be functionally replaced by the TIRAP $\mathrm{PIP}_{2}$ binding domain (Hermann 2009), membrane recruitment of TIRAP fused to the SLP65 R-to-A variant did not exceed the membrane recruitment of the negative control. Since the experiment worked technically, functionally there might be cooperation between the $\mathrm{PIP}_{2}$ binding domain of TIRAP (and SLP65 N-terminus?) and SLP65 proline-arginine motifs explaining why the TIRAP membrane anchor did not place proline-arginine exchanged SLP65 to the membrane. Hence, another membrane anchor has to be analysed in order to reveal the functional mechanism.

In line with the data showing that CIN85 is the dominant ligand of SLP65 in BCR-induced $\mathrm{Ca}^{2+}$ signalling, CIN85 rather than CD2AP would provide SLP65 with access to the plasma membrane. Moreover, since SLP65 resided in BCR microclusters as well, which is in accordance with its direct binding to Iga (Engels et al. 2001; Oellerich et al. 2011) CIN85 could have an additional BCR-targeting function for SLP65 rather than general plasma membrane localisation. This is further supported by the notion that BCR-targeting of SLP65 was sufficient to bypass CIN85 binding for BCR-induced $\mathrm{Ca}^{2+}$ mobilisation. BCR-targeted SLP65 then would be directly accessible for phosphorylation by Syk, increasing the efficiency of BCR signal transduction. Previous studies described that phosphorylation of BCR effector proteins did depend on the BCR as organiser of an unidentified preformed BCR transducer complex (Wienands et al. 1996). In this line preformation of SLP65 with its BCR-targeting device CIN85, would allow a fast and efficient BCR signal throughput, explaining the delay in 
the onset of BCR-induced $\mathrm{Ca}^{2+}$ mobilisation in cells, in which the CIN85/SLP65 module is not formed. Together with the evidence that the proline-arginine motifs provide a plasma membrane localisation signal, the dominant role of CIN85 in BCR-induced $\mathrm{Ca}^{2+}$ signalling and the colocalisation of CIN85 and SLP65 to BCR-containing microcluster it is rather CIN85 and SLP65 that constitute a preformed signal transducer module, that localises, not to the plasma membrane in general, but to BCR-rich subdomains of it.

To further proof the importance of a CIN85 and SLP65 module, chimeric proteins encompassing parts of CIN85 or CD2AP fused to mutant SLP65 could be analysed for their signalling competence. Another tool for studying this issue would be to dimerise engineered CIN85 and the SLP65 R-to-A variant with a cross-linker. Thereby functionality of a CIN85/SLP65 module can be measured in s/p65 cells, even in the presence of endogenous CIN85. Performing these experiments with CD2AP as well will allow further discrimination of CIN85 vs. CD2AP function with regard to SLP65.
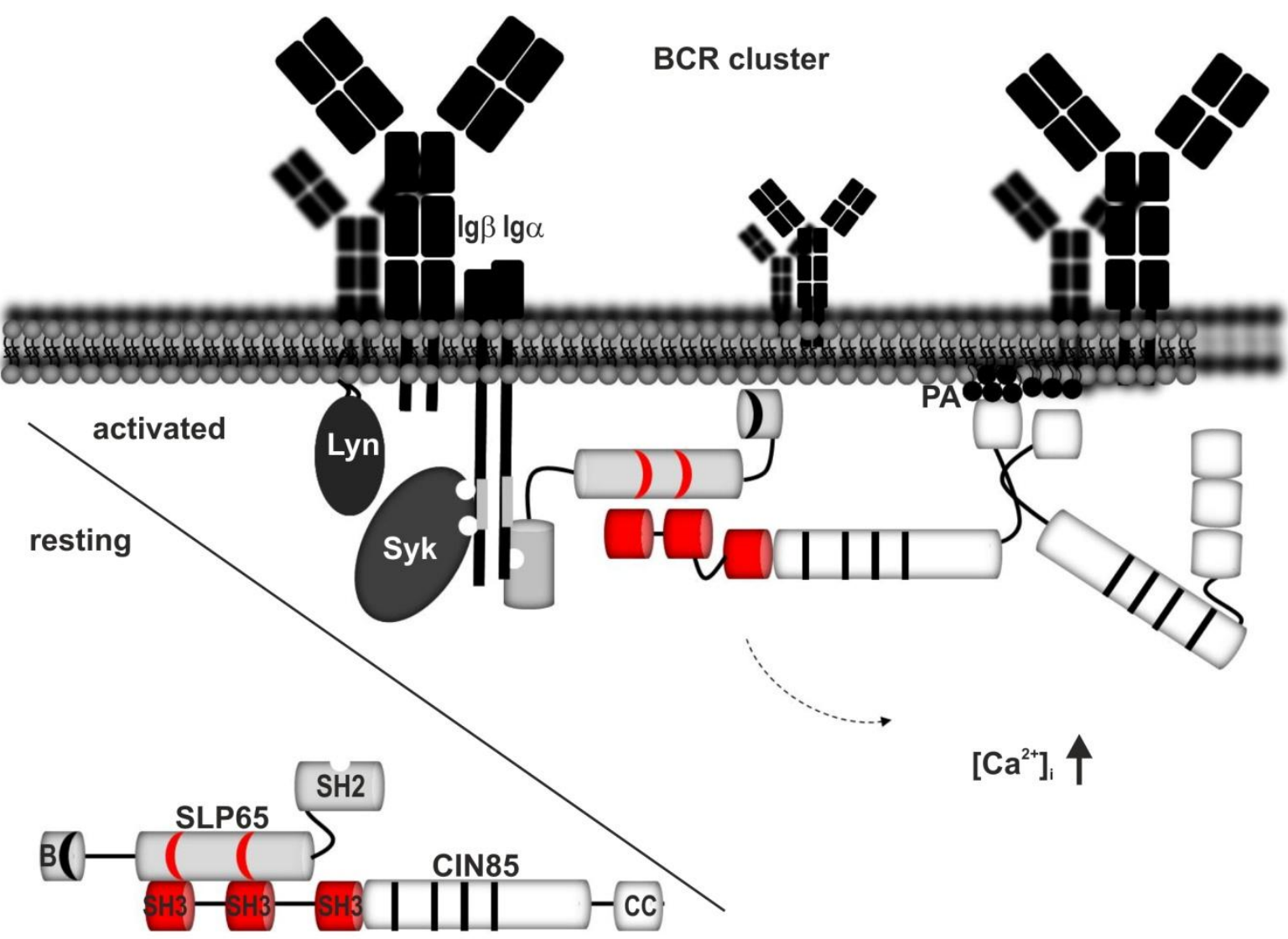

Figure 4.3: Model of the preformed CIN85/SLP65 BCR transducer module in the initiation of $\mathrm{Ca}^{2+}$ signalling.

CIN85 and SLP65 reside in the cytosol of resting B cells as a preformed CIN85/SLP65 complex. Upon stimulation of the BCR a Lyn-mediated signal leads to translocation of the CIN85/SLP65 complex to BCR-containing microclusters. Membrane localisation of CIN85 requires its coiled coil domain to interact with phosphatidic acid (PA). The CIN85/SLP65 complex is further stabilised by the interaction of the SLP65 SH2 domain with Iga and the basic effector domain (B) with phospholipids in the plasma membrane. At the BCR SLP65 is phosphorylated by Syk allowing a rapid onset and efficient course of $\mathrm{BCR}$-induced $\mathrm{Ca}^{2+}$ mobilisation. 


\subsection{Outlook}

Based on the findings of this thesis and the B cell specific cin85/- mice (Kometani et al. 2011) as well as on a model for the function of the T cell immunological synapse (Lee et al. 2003), I will discuss a function of CIN85 during formation of the B cell immunological synapse as an outlook. In this model CIN85 facilitates BCR signalling by preformation with SLP65 and subsequent location of SLP65-based signalosomes directly to BCR microclusters. This mechanism might be of particular importance when the activation of the $B$ cell relies specifically on BCR signal transduction and when the antigenic load is relatively low. Besides supporting the formation of microclusters, CIN85 might even increase the number of $\mathrm{BCR} /$ antigen clusters which then translates in enhanced $\mathrm{Ca}^{2+}$ signalling. In the subsequent contraction phase CIN85 might be involved in clustering of the BCR/antigen complexes. Thereby the signal's own decline is induced resulting in the degradation of BCRs.

The cytoskeleton is getting more and more into the focus of BCR signalling (Viola and Gupta 2007; Batista et al. 2010), because it restricts BCR diffusion and thereby signalling in resting B cells (Treanor et al. 2009), allows morphological changes during spreading and has a pivotal role in gathering of antigen into the immunological synapse (Schnyder et al. 2011). CIN85/CD2AP are multiply linked to this cellular microfilament (Kirsch et al. 1999; Hutchings et al. 2003; Lynch et al. 2003; Gaidos et al. 2007). Exploration of the function of the CIN85/CD2AP family of adaptor proteins in B cells has just started (Niiro et al.; Kometani et al. 2011; Oellerich et al. 2011; Niiro et al. 2012) and future studies should be linked to the cytoskeleton. This will involve sophisticated imaging technologies and the use of CIN85/CD2AP-double deficient B cells. 


\section{Summary and conclusions}

The immune system is an extraordinary evolutionary achievement in protecting the organism from the fatal consequences of pathogenic invasion. One powerful executive molecule of the body's defence to pathogen is the antibody. Antibodies are produced by $B$ cells as the soluble form of the $\mathrm{mlg}$ on the B cell's surface. Antigen-induced BCR signalling is the prerequisite for antigen-specific antibody secretion. Early BCR signal transduction is characterised by the assembly of BCR effector proteins into multimeric complexes and translocation of these signalosomes to BCR-containing microdomains in the plasma membrane. The $\mathrm{Ca}^{2+}$ initiation complex is such a signalosome that has to be built and targeted to the plasma membrane by the central adaptor protein SLP65. Therefore SLP65 has to be phosphorylated and recruited to the plasma membrane. To elucidate the mechanism of SLP65 membrane localisation, the interaction partners of SLP65 were analysed by a mass spectrometric approach in our group. The adaptor proteins CIN85 and CD2AP were identified as steady interaction partners of SLP65. Disruption of this preassociation by the exchange of critical amino acids in SLP65 resulted in a defect in SLP65 phosphorylation and $\mathrm{Ca}^{2+}$ mobilisation in DT40 and primary B cells after BCR stimulation. Moreover, it was discussed earlier in our group that preformed, BCR signallingindependent complexes must exist to allow rapid BCR signal initiation, but their nature remained elusive. Hence, the following questions arose: do CIN85 and CD2AP have a role in BCR signalling and do they together with SLP65 comprise a preformed signal transducer complex in BCR-induced $\mathrm{Ca}^{2+}$ mobilisation? And how then do they support SLP65 function in BCR signal transduction?

In this thesis a positive function of CIN85 in the onset and strength of BCR-induced $\mathrm{Ca}^{2+}$ mobilisation in DT40 B cells could be shown. However, CD2AP could partially replace CIN85 function in this respect, because a profound defect in BCR-induced $\mathrm{Ca}^{2+}$ mobilisation was only observable upon combined reduction of cd2ap and cin85 expression. Hence, ablation of CIN85 and CD2AP displayed a similar impairment of $\mathrm{Ca}^{2+}$ flux as the SLP65 variant that did not bind CIN85 and CD2AP. Biochemical analysis revealed that the interaction of CIN85/CD2AP with SLP65 is mediated by the SH3 domains of CIN85/CD2AP and the second and third proline-arginine motif in SLP65. Using live cell microscopy techniques I demonstrated that CIN85 and CD2AP were recruited to the plasma membrane upon stimulation of the BCR, but used different anchoring modes. While CD2AP required its coiled coil domain possibly to interact with the cytoskeleton, CIN85 needed its SH3 as well as its coiled coil domains to anchor to the plasma membrane. Interestingly, the very same domains of CIN85 necessary for BCR-induced $\mathrm{Ca}^{2+}$ signalling provided CIN85 with access to the 
plasma membrane. This indicates that the function of CIN85 in BCR-induced $\mathrm{Ca}^{2+}$ mobilisation is connected to its ability to translocate to the plasma membrane. High resolution analysis of membrane localisation using total internal reflection microscopy revealed that CIN85 colocalised with BCR-containing microclusters upon BCR engagement, while only very few CD2AP molecules did. CIN85 plasma membrane translocation and presumably BCR colocalisation required Lyn, but not Syk kinase activity. Importantly, the binding of CIN85 to SLP65 could be bypassed by providing SLP65 with direct access to the BCR. Together with previous data from our group my data provide evidence for the existence of a preformed BCR signal transducer module of CIN85 and SLP65 operating in BCR signalling. The scaffold CIN85 targets its already bound ligand SLP65 directly to BCRcontaining microclusters explaining the efficiency of BCR signal transduction. The identification of a CIN85/SLP65 transducer complex contributes to the knowledge of early BCR activation with respect to preformed and BCR-targeted signalosomes and the importance of adaptor proteins in the kinetic and magnitude of the BCR signal. The exploration of CIN85 in B cells has just started and this thesis is one of the first descriptions of the importance of this scaffold in BCR signal transduction. The functional importance of CIN85 is further corroborated by the fact that CIN85 is indispensable in the generation of T cell-independent antibody responses (Kometani et al. 2011). The failure to produce antibodies is manifested in medicine by humoral immunodeficiencies, which results in livelong suffering from infections with eventually fatal consequences. With my studies I hope to contribute to future investigations aiming at understanding and treating B cell-related diseases. 


\section{References}

Abudula, A., A. Grabbe, M. Brechmann, C. Polaschegg, N. Herrmann, I. Goldbeck, K. Dittmann and J. Wienands (2007). "SLP-65 signal transduction requires Src homology 2 domain-mediated membrane anchoring and a kinase-independent adaptor function of Syk." J Biol Chem 282(39): 29059-66.

Aman, M. J. and K. S. Ravichandran (2000). "A requirement for lipid rafts in B cell receptor induced $\mathrm{Ca}(2+)$ flux." Curr Biol 10(7): 393-6.

Amzel, L. M., R. J. Poljak, F. Saul, J. M. Varga and F. F. Richards (1974). "The three dimensional structure of a combining region-ligand complex of immunoglobulin NEW at 3.5-A resolution." Proc Natl Acad Sci U S A 71(4): 1427-30.

Ausubel, F. M., R. Brent, R. E. Kingdom, D. M. Moore, J. G. Seidman, J. A. Smith and K. Struhl (1995). "Current Protocols in Molecular Biology."

Baba, T. W. and E. H. Humphries (1984). "Avian leukosis virus infection: analysis of viremia and DNA integration in susceptible and resistant chicken lines." J Virol 51(1): 123-30.

Baba, Y., S. Hashimoto, M. Matsushita, D. Watanabe, T. Kishimoto, T. Kurosaki and S. Tsukada (2001). "BLNK mediates Syk-dependent Btk activation." Proc Natl Acad Sci U S A 98(5): 2582-6.

Baba, Y., K. Hayashi, Y. Fujii, A. Mizushima, H. Watarai, M. Wakamori, T. Numaga, Y. Mori, M. lino, M. Hikida and T. Kurosaki (2006). "Coupling of STIM1 to store-operated Ca2+ entry through its constitutive and inducible movement in the endoplasmic reticulum." Proc Natl Acad Sci U S A 103(45): 16704-9.

Batista, F. D., D. Iber and M. S. Neuberger (2001). "B cells acquire antigen from target cells after synapse formation." Nature 411(6836): 489-94.

Batista, F. D., B. Treanor and N. E. Harwood (2010). "Visualizing a role for the actin cytoskeleton in the regulation of B-cell activation." Immunol Rev 237(1): 191-204.

Bohnenberger, H., T. Oellerich, M. Engelke, H. H. Hsiao, H. Urlaub and J. Wienands (2011). "Complex phosphorylation dynamics control the composition of the Syk interactome in B cells." Eur J Immunol 41(6): 1550-62.

Bolland, S., R. N. Pearse, T. Kurosaki and J. V. Ravetch (1998). "SHIP modulates immune receptor responses by regulating membrane association of Btk." Immunity 8(4): 50916.

Bonilla, F. A., R. M. Fujita, V. I. Pivniouk, A. C. Chan and R. S. Geha (2000). "Adapter proteins SLP-76 and BLNK both are expressed by murine macrophages and are linked to signaling via Fcgamma receptors I and II/III." Proc Natl Acad Sci U S A 97(4): 1725-30. 
Borinstein, S. C., M. A. Hyatt, V. W. Sykes, R. E. Straub, S. Lipkowitz, J. Boulter and O. Bogler (2000). "SETA is a multifunctional adapter protein with three $\mathrm{SH} 3$ domains that binds Grb2, Cbl, and the novel SB1 proteins." Cell Signal 12(11-12): 769-79.

Brdicka, T., J. Cerny and V. Horejsi (1998). "T cell receptor signalling results in rapid tyrosine phosphorylation of the linker protein LAT present in detergent-resistant membrane microdomains." Biochem Biophys Res Commun 248(2): 356-60.

Bubeck Wardenburg, J., R. Pappu, J. Y. Bu, B. Mayer, J. Chernoff, D. Straus and A. C. Chan (1998). "Regulation of PAK activation and the T cell cytoskeleton by the linker protein SLP-76." Immunity 9(5): 607-16.

Buchman, V. L., C. Luke, E. B. Borthwick, I. Gout and N. Ninkina (2002). "Organization of the mouse Ruk locus and expression of isoforms in mouse tissues." Gene 295(1): 13-17.

Buchse, T., N. Horras, E. Lenfert, G. Krystal, S. Korbel, M. Schumann, E. Krause, S. Mikkat and M. Tiedge (2011). "CIN85 interacting proteins in B cells-specific role for SHIP-1." Mol Cell Proteomics 10(10): M110 006239.

Bunnell, S. C., V. Kapoor, R. P. Trible, W. Zhang and L. E. Samelson (2001). "Dynamic actin polymerization drives $T$ cell receptor-induced spreading: a role for the signal transduction adaptor LAT." Immunity 14(3): 315-29.

Burack, W. R., A. M. Cheng and A. S. Shaw (2002). "Scaffolds, adaptors and linkers of TCR signaling: theory and practice." Curr Opin Immunol 14(3): 312-6.

Burkhardt, A. L., M. Brunswick, J. B. Bolen and J. J. Mond (1991). "Anti-immunoglobulin stimulation of B lymphocytes activates src-related protein-tyrosine kinases." Proc Natl Acad Sci U S A 88(16): 7410-4.

Burnet, F. M. (1959). "The Clonal Selection Theory of Aquired Immunity." Cambridge University Press.

Cambier, J. C. (1995). "Antigen and Fc receptor signaling. The awesome power of the

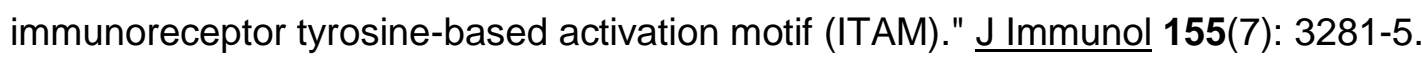

Campbell, K. S. and J. C. Cambier (1990). "B lymphocyte antigen receptors (mlg) are noncovalently associated with a disulfide linked, inducibly phosphorylated glycoprotein complex." Embo J 9(2): 441-8.

Campbell, M. A. and B. M. Sefton (1992). "Association between B-lymphocyte membrane immunoglobulin and multiple members of the Src family of protein tyrosine kinases." Mol Cell Biol 12(5): 2315-21.

Chapman, S. A. and A. R. Asthagiri (2009). "Quantitative effect of scaffold abundance on signal propagation." Mol Syst Biol 5: 313.

Cheng, P. C., M. L. Dykstra, R. N. Mitchell and S. K. Pierce (1999). "A role for lipid rafts in B cell antigen receptor signaling and antigen targeting." J Exp Med 190(11): 1549-60. 
Chiu, C. W., M. Dalton, M. Ishiai, T. Kurosaki and A. C. Chan (2002). "BLNK: molecular scaffolding through 'cis'-mediated organization of signaling proteins." Embo J 21(23): 6461-72.

Clark, M. R., K. S. Campbell, A. Kazlauskas, S. A. Johnson, M. Hertz, T. A. Potter, C. Pleiman and J. C. Cambier (1992). "The B cell antigen receptor complex: association of Ig-alpha and Ig-beta with distinct cytoplasmic effectors." Science 258(5079): 123-6.

Corey, S. J., P. M. Dombrosky-Ferlan, S. Zuo, E. Krohn, A. D. Donnenberg, P. Zorich, G. Romero, M. Takata and T. Kurosaki (1998). "Requirement of Src kinase Lyn for induction of DNA synthesis by granulocyte colony-stimulating factor." $\underline{\mathrm{J} \text { Biol Chem }}$ 273(6): 3230-5.

Cullen, B. R. (2005). "RNAi the natural way." Nat Genet 37(11): 1163-5.

Davis, D. M., I. Chiu, M. Fassett, G. B. Cohen, O. Mandelboim and J. L. Strominger (1999). "The human natural killer cell immune synapse." Proc Natl Acad Sci U S A 96(26): 15062-7.

Depoil, D., S. Fleire, B. L. Treanor, M. Weber, N. E. Harwood, K. L. Marchbank, V. L. Tybulewicz and F. D. Batista (2008). "CD19 is essential for B cell activation by promoting $B$ cell receptor-antigen microcluster formation in response to membranebound ligand." Nat Immunol 9(1): 63-72.

Dickins, R. A., M. T. Hemann, J. T. Zilfou, D. R. Simpson, I. Ibarra, G. J. Hannon and S. W. Lowe (2005). "Probing tumor phenotypes using stable and regulated synthetic microRNA precursors." Nat Genet 37(11): 1289-95.

Dikic, I. (2002). "CIN85/CMS family of adaptor molecules." FEBS Lett 529(1): 110-5.

Dolmetsch, R. E., R. S. Lewis, C. C. Goodnow and J. I. Healy (1997). "Differential activation of transcription factors induced by $\mathrm{Ca} 2+$ response amplitude and duration." Nature 386(6627): 855-8.

Dustin, M. L., M. W. Olszowy, A. D. Holdorf, J. Li, S. Bromley, N. Desai, P. Widder, F. Rosenberger, P. A. van der Merwe, P. M. Allen and A. S. Shaw (1998). "A novel adaptor protein orchestrates receptor patterning and cytoskeletal polarity in T-cell contacts." Cell 94(5): 667-77.

Ehrlich, P. (1900). "The Croonian Lecture to the Royal Society." Proc. R. Soc. London 66: 424-448.

Emi, N., T. Friedmann and J. K. Yee (1991). "Pseudotype formation of murine leukemia virus with the G protein of vesicular stomatitis virus." J Virol 65(3): 1202-7.

Engelke, M., N. Engels, K. Dittmann, B. Stork and J. Wienands (2007). "Ca(2+) signaling in antigen receptor-activated B lymphocytes." Immunol Rev 218: 235-46.

Engels, N., M. Engelke and J. Wienands (2008). "Conformational plasticity and navigation of signaling proteins in antigen-activated B lymphocytes." Adv Immunol 97: 251-81. 
Engels, N., B. Wollscheid and J. Wienands (2001). "Association of SLP-65/BLNK with the B cell antigen receptor through a non-ITAM tyrosine of Ig-alpha." Eur J Immunol 31(7): 2126-34.

Fagarasan, S. and T. Honjo (2000). "T-Independent immune response: new aspects of B cell biology." Science 290(5489): 89-92.

Falasca, M., S. K. Logan, V. P. Lehto, G. Baccante, M. A. Lemmon and J. Schlessinger (1998). "Activation of phospholipase C gamma by PI 3-kinase-induced PH domainmediated membrane targeting." Embo J 17(2): 414-22.

Feng, S., J. K. Chen, H. Yu, J. A. Simon and S. L. Schreiber (1994). "Two binding orientations for peptides to the Src SH3 domain: development of a general model for SH3-ligand interactions." Science 266(5188): 1241-7.

Feng, S., C. Kasahara, R. J. Rickles and S. L. Schreiber (1995). "Specific interactions outside the proline-rich core of two classes of Src homology 3 ligands." Proc Natl Acad Sci U S A 92(26): 12408-15.

Feske, S., Y. Gwack, M. Prakriya, S. Srikanth, S. H. Puppel, B. Tanasa, P. G. Hogan, R. S. Lewis, M. Daly and A. Rao (2006). "A mutation in Orai1 causes immune deficiency by abrogating CRAC channel function." Nature 441(7090): 179-85.

Finniss, S., A. Movsisyan, C. Billecke, M. Schmidt, L. Randazzo, B. Chen and O. Bogler (2004). "Studying protein isoforms of the adaptor SETA/CIN85/Ruk with monoclonal antibodies." Biochem Biophys Res Commun 325(1): 174-82.

Fleire, S. J., J. P. Goldman, Y. R. Carrasco, M. Weber, D. Bray and F. D. Batista (2006). "B cell ligand discrimination through a spreading and contraction response." Science 312(5774): 738-41.

Fu, C., C. W. Turck, T. Kurosaki and A. C. Chan (1998). "BLNK: a central linker protein in B cell activation." Immunity 9(1): 93-103.

Futterer, K., J. Wong, R. A. Grucza, A. C. Chan and G. Waksman (1998). "Structural basis for Syk tyrosine kinase ubiquity in signal transduction pathways revealed by the crystal structure of its regulatory $\mathrm{SH} 2$ domains bound to a dually phosphorylated ITAM peptide." J Mol Biol 281(3): 523-37.

Gaidos, G., S. Soni, D. J. Oswald, P. A. Toselli and K. H. Kirsch (2007). "Structure and function analysis of the CMS/CIN85 protein family identifies actin-bundling properties and heterotypic-complex formation." J Cell Sci 120(Pt 14): 2366-77.

Gavin, A. C., P. Aloy, P. Grandi, R. Krause, M. Boesche, M. Marzioch, C. Rau, L. J. Jensen, S. Bastuck, B. Dumpelfeld, A. Edelmann, M. A. Heurtier, V. Hoffman, C. Hoefert, K. Klein, M. Hudak, A. M. Michon, M. Schelder, M. Schirle, M. Remor, T. Rudi, S. Hooper, A. Bauer, T. Bouwmeester, G. Casari, G. Drewes, G. Neubauer, J. M. Rick, 
B. Kuster, P. Bork, R. B. Russell and G. Superti-Furga (2006). "Proteome survey reveals modularity of the yeast cell machinery." Nature 440(7084): 631-6.

Gavin, A. C., M. Bosche, R. Krause, P. Grandi, M. Marzioch, A. Bauer, J. Schultz, J. M. Rick, A. M. Michon, C. M. Cruciat, M. Remor, C. Hofert, M. Schelder, M. Brajenovic, H. Ruffner, A. Merino, K. Klein, M. Hudak, D. Dickson, T. Rudi, V. Gnau, A. Bauch, S. Bastuck, B. Huhse, C. Leutwein, M. A. Heurtier, R. R. Copley, A. Edelmann, E. Querfurth, V. Rybin, G. Drewes, M. Raida, T. Bouwmeester, P. Bork, B. Seraphin, B. Kuster, G. Neubauer and G. Superti-Furga (2002). "Functional organization of the yeast proteome by systematic analysis of protein complexes." Nature 415(6868): 1417.

Glick, B., Chang, T.S., Jaap, R.G. (1956). "The bursa of Fabricius and antibody production." Poult. Sci.(35): 224.

Goitsuka, R., Y. Fujimura, H. Mamada, A. Umeda, T. Morimura, K. Uetsuka, K. Doi, S. Tsuji and D. Kitamura (1998). "BASH, a novel signaling molecule preferentially expressed in B cells of the bursa of Fabricius." J Immunol 161(11): 5804-8.

Gout, I., G. Middleton, J. Adu, N. N. Ninkina, L. B. Drobot, V. Filonenko, G. Matsuka, A. M. Davies, M. Waterfield and V. L. Buchman (2000). "Negative regulation of PI 3-kinase by Ruk, a novel adaptor protein." Embo J 19(15): 4015-25.

Grabbe, A. and J. Wienands (2006). "Human SLP-65 isoforms contribute differently to activation and apoptosis of B lymphocytes." Blood 108(12): 3761-8.

Grande, S. M., G. Bannish, E. M. Fuentes-Panana, E. Katz and J. G. Monroe (2007). "Tonic B-cell and viral ITAM signaling: context is everything." Immunol Rev 218: 214-34.

Guagliardi, L. E., B. Koppelman, J. S. Blum, M. S. Marks, P. Cresswell and F. M. Brodsky (1990). "Co-localization of molecules involved in antigen processing and presentation in an early endocytic compartment." Nature 343(6254): 133-9.

Haglund, K., N. Shimokawa, I. Szymkiewicz and I. Dikic (2002). "Cbl-directed monoubiquitination of CIN85 is involved in regulation of ligand-induced degradation of EGF receptors." Proc Natl Acad Sci U S A 99(19): 12191-6.

Hashimoto, A., H. Okada, A. Jiang, M. Kurosaki, S. Greenberg, E. A. Clark and T. Kurosaki (1998). "Involvement of guanosine triphosphatases and phospholipase C-gamma2 in extracellular signal-regulated kinase, c-Jun NH2-terminal kinase, and p38 mitogenactivated protein kinase activation by the B cell antigen receptor." J Exp Med 188(7): 1287-95.

Hashimoto, S., A. Iwamatsu, M. Ishiai, K. Okawa, T. Yamadori, M. Matsushita, Y. Baba, T. Kishimoto, T. Kurosaki and S. Tsukada (1999). "Identification of the SH2 domain binding protein of Bruton's tyrosine kinase as BLNK--functional significance of Btk- 
SH2 domain in B-cell antigen receptor-coupled calcium signaling." Blood 94(7): 235764.

Hawley, R. G., F. H. Lieu, A. Z. Fong and T. S. Hawley (1994). "Versatile retroviral vectors for potential use in gene therapy." Gene Ther 1(2): 136-8.

Hayakawa, K., M. Asano, S. A. Shinton, M. Gui, D. Allman, C. L. Stewart, J. Silver and R. R. Hardy (1999). "Positive selection of natural autoreactive B cells." Science 285(5424): 113-6.

Hayashi, K., R. Nittono, N. Okamoto, S. Tsuji, Y. Hara, R. Goitsuka and D. Kitamura (2000). "The $\mathrm{B}$ cell-restricted adaptor $\mathrm{BASH}$ is required for normal development and antigen receptor-mediated activation of B cells." Proc Natl Acad Sci U S A 97(6): 2755-60.

Hermann, N. (2009). "Kooperation funktioneller Domänen des Adapterproteins SLP-65 für die Ca2+-Antwort in B-Lymphocyten."

Hitomi, T., S. Yanagi, R. Inatome and H. Yamamura (1999). "Cross-linking of the B cell receptor induces activation of phospholipase $D$ through Syk, Btk and phospholipase C-gamma2." FEBS Lett 445(2-3): 371-4.

Hoek, K. L., P. Antony, J. Lowe, N. Shinners, B. Sarmah, S. R. Wente, D. Wang, R. M. Gerstein and W. N. Khan (2006). "Transitional B cell fate is associated with developmental stage-specific regulation of diacylglycerol and calcium signaling upon B cell receptor engagement." J Immunol 177(8): 5405-13.

Hombach, J., T. Tsubata, L. Leclercq, H. Stappert and M. Reth (1990). "Molecular components of the B-cell antigen receptor complex of the IgM class." Nature 343(6260): 760-2.

Hoth, M. and R. Penner (1992). "Depletion of intracellular calcium stores activates a calcium current in mast cells." Nature 355(6358): 353-6.

Huang, G. N., W. Zeng, J. Y. Kim, J. P. Yuan, L. Han, S. Muallem and P. F. Worley (2006). "STIM1 carboxyl-terminus activates native SOC, I(crac) and TRPC1 channels." $\underline{\mathrm{Nat}}$ Cell Biol 8(9): 1003-10.

Hutchings, N. J., N. Clarkson, R. Chalkley, A. N. Barclay and M. H. Brown (2003). "Linking the $T$ cell surface protein CD2 to the actin-capping protein CAPZ via CMS and CIN85." J Biol Chem 278(25): 22396-403.

Imai, C., M. E. Ross, G. Reid, E. Coustan-Smith, K. R. Schultz, C. H. Pui, J. R. Downing and D. Campana (2004). "Expression of the adaptor protein BLNK/SLP-65 in childhood acute lymphoblastic leukemia." Leukemia 18(5): 922-5.

Ishiai, M., M. Kurosaki, K. Inabe, A. C. Chan, K. Sugamura and T. Kurosaki (2000). "Involvement of LAT, Gads, and Grb2 in compartmentation of SLP-76 to the plasma membrane." J Exp Med 192(6): 847-56. 
Ishiai, M., M. Kurosaki, R. Pappu, K. Okawa, I. Ronko, C. Fu, M. Shibata, A. Iwamatsu, A. C. Chan and T. Kurosaki (1999a). "BLNK required for coupling Syk to PLC gamma 2 and Rac1-JNK in B cells." Immunity 10(1): 117-25.

Ishiai, M., H. Sugawara, M. Kurosaki and T. Kurosaki (1999b). "Cutting edge: association of phospholipase C-gamma 2 Src homology 2 domains with BLNK is critical for B cell antigen receptor signaling." $\mathrm{J}$ Immunol 163(4): 1746-9.

Johnson, G. and T. T. Wu (2000). "Kabat database and its applications: 30 years after the first variability plot." Nucleic Acids Res 28(1): 214-8.

Jumaa, H., L. Bossaller, K. Portugal, B. Storch, M. Lotz, A. Flemming, M. Schrappe, V. Postila, P. Riikonen, J. Pelkonen, C. M. Niemeyer and M. Reth (2003). "Deficiency of the adaptor SLP-65 in pre-B-cell acute lymphoblastic leukaemia." Nature 423(6938): 452-6.

Jumaa, H., B. Wollscheid, M. Mitterer, J. Wienands, M. Reth and P. J. Nielsen (1999). "Abnormal development and function of $B$ lymphocytes in mice deficient for the signaling adaptor protein SLP-65." Immunity 11(5): 547-54.

Kabak, S., B. J. Skaggs, M. R. Gold, M. Affolter, K. L. West, M. S. Foster, K. Siemasko, A. C. Chan, R. Aebersold and M. R. Clark (2002). "The direct recruitment of BLNK to immunoglobulin alpha couples the B-cell antigen receptor to distal signaling pathways." Mol Cell Biol 22(8): 2524-35.

Kagan, J. C. and R. Medzhitov (2006). "Phosphoinositide-mediated adaptor recruitment controls Toll-like receptor signaling." Cell 125(5): 943-55.

Kimura, T., H. Sakamoto, E. Appella and R. P. Siraganian (1996). "Conformational changes induced in the protein tyrosine kinase p72syk by tyrosine phosphorylation or by binding of phosphorylated immunoreceptor tyrosine-based activation motif peptides." Mol Cell Biol 16(4): 1471-8.

King, L. B. and J. G. Monroe (2000). "Immunobiology of the immature B cell: plasticity in the B-cell antigen receptor-induced response fine tunes negative selection." Immunol Rev 176: 86-104.

Kirsch, K. H., M. M. Georgescu, S. Ishimaru and H. Hanafusa (1999). "CMS: an adapter molecule involved in cytoskeletal rearrangements." Proc Natl Acad Sci U S A 96(11): 6211-6.

Kirsch, K. H., M. M. Georgescu, T. Shishido, W. Y. Langdon, R. B. Birge and H. Hanafusa (2001). "The adapter type protein CMS/CD2AP binds to the proto-oncogenic protein c-Cbl through a tyrosine phosphorylation-regulated Src homology 3 domain interaction." J Biol Chem 276(7): 4957-63. 
Kitaura, Y., I. K. Jang, Y. Wang, Y. C. Han, T. Inazu, E. J. Cadera, M. Schlissel, R. R. Hardy and H. Gu (2007). "Control of the B cell-intrinsic tolerance programs by ubiquitin ligases Cbl and Cbl-b." Immunity 26(5): 567-78.

Kobayashi, S., A. Sawano, Y. Nojima, M. Shibuya and Y. Maru (2004). "The c-CbI/CD2AP complex regulates VEGF-induced endocytosis and degradation of Flt-1 (VEGFR-1)." Faseb J 18(7): 929-31.

Kohler, F., B. Storch, Y. Kulathu, S. Herzog, S. Kuppig, M. Reth and H. Jumaa (2005). "A leucine zipper in the $\mathrm{N}$ terminus confers membrane association to SLP-65." Nat Immunol 6(2): 204-10.

Kometani, K., T. Yamada, Y. Sasaki, T. Yokosuka, T. Saito, K. Rajewsky, M. Ishiai, M. Hikida and T. Kurosaki (2011). "CIN85 drives B cell responses by linking BCR signals to the canonical NF-kappaB pathway." J Exp Med 208(7): 1447-57.

Koretzky, G. A., F. Abtahian and M. A. Silverman (2006). "SLP76 and SLP65: complex regulation of signalling in lymphocytes and beyond." Nat Rev Immunol 6(1): 67-78.

Kowanetz, K., K. Husnjak, D. Holler, M. Kowanetz, P. Soubeyran, D. Hirsch, M. H. Schmidt, K. Pavelic, P. De Camilli, P. A. Randazzo and I. Dikic (2004). "CIN85 associates with multiple effectors controlling intracellular trafficking of epidermal growth factor receptors." Mol Biol Cell 15(7): 3155-66.

Kowanetz, K., I. Szymkiewicz, K. Haglund, M. Kowanetz, K. Husnjak, J. D. Taylor, P. Soubeyran, U. Engstrom, J. E. Ladbury and I. Dikic (2003). "Identification of a novel proline-arginine motif involved in CIN85-dependent clustering of $\mathrm{Cbl}$ and downregulation of epidermal growth factor receptors." J Biol Chem 278(41): 39735-46.

Kulathu, Y., E. Hobeika, G. Turchinovich and M. Reth (2008). "The kinase Syk as an adaptor controlling sustained calcium signalling and B-cell development." Embo J 27(9): 1333-44.

Kurakin, A. V., S. Wu and D. E. Bredesen (2003). "Atypical recognition consensus of CIN85/SETA/Ruk SH3 domains revealed by target-assisted iterative screening." $\underline{J}$ Biol Chem 278(36): 34102-9.

Kurosaki, T., S. A. Johnson, L. Pao, K. Sada, H. Yamamura and J. C. Cambier (1995). "Role of the Syk autophosphorylation site and $\mathrm{SH} 2$ domains in $\mathrm{B}$ cell antigen receptor signaling." J Exp Med 182(6): 1815-23.

Kurosaki, T. and M. Kurosaki (1997). "Transphosphorylation of Bruton's tyrosine kinase on tyrosine 551 is critical for B cell antigen receptor function." $\underline{J}$ Biol Chem $\mathbf{2 7 2 ( 2 5 ) :}$ 15595-8.

Kurosaki, T., A. Maeda, M. Ishiai, A. Hashimoto, K. Inabe and M. Takata (2000). "Regulation of the phospholipase C-gamma2 pathway in B cells." Immunol Rev 176: 19-29. 
Kurosaki, T., M. Takata, Y. Yamanashi, T. Inazu, T. Taniguchi, T. Yamamoto and H. Yamamura (1994). "Syk activation by the Src-family tyrosine kinase in the B cell receptor signaling." J Exp Med 179(5): 1725-9.

Laemmli, U. K. (1970). "Cleavage of structural proteins during the assembly of the head of bacteriophage T4." Nature 227(5259): 680-5.

Lanzavecchia, A. (1985). "Antigen-specific interaction between T and B cells." Nature 314(6011): 537-9.

Lee, K. H., A. R. Dinner, C. Tu, G. Campi, S. Raychaudhuri, R. Varma, T. N. Sims, W. R. Burack, H. Wu, J. Wang, O. Kanagawa, M. Markiewicz, P. M. Allen, M. L. Dustin, A. K. Chakraborty and A. S. Shaw (2003). "The immunological synapse balances T cell receptor signaling and degradation." Science 302(5648): 1218-22.

Lehtonen, S., A. Ora, V. M. Olkkonen, L. Geng, M. Zerial, S. Somlo and E. Lehtonen (2000). "In vivo interaction of the adapter protein CD2-associated protein with the type 2 polycystic kidney disease protein, polycystin-2." J Biol Chem 275(42): 32888-93.

Levchenko, A., J. Bruck and P. W. Sternberg (2000). "Scaffold proteins may biphasically affect the levels of mitogen-activated protein kinase signaling and reduce its threshold properties." Proc Natl Acad Sci U S A 97(11): 5818-23.

Lim, W. A., F. M. Richards and R. O. Fox (1994). "Structural determinants of peptide-binding orientation and of sequence specificity in SH3 domains." Nature 372(6504): 375-9.

Lin, J. and L. B. Justement (1992). "The MB-1/B29 heterodimer couples the B cell antigen receptor to multiple src family protein tyrosine kinases." J Immunol 149(5): 1548-55.

Liu, Q., D. Berry, P. Nash, T. Pawson, C. J. McGlade and S. S. Li (2003). "Structural basis for specific binding of the Gads SH3 domain to an RxxK motif-containing SLP-76 peptide: a novel mode of peptide recognition." Mol Cell 11(2): 471-81.

Liu, S. K., N. Fang, G. A. Koretzky and C. J. McGlade (1999). "The hematopoietic-specific adaptor protein gads functions in T-cell signaling via interactions with the SLP-76 and LAT adaptors." Curr Biol 9(2): 67-75.

Lowik, M. M., P. J. Groenen, I. Pronk, M. R. Lilien, R. Goldschmeding, H. B. Dijkman, E. N. Levtchenko, L. A. Monnens and L. P. van den Heuvel (2007). "Focal segmental glomerulosclerosis in a patient homozygous for a CD2AP mutation." Kidney Int 72(10): 1198-203.

Luik, R. M., M. M. Wu, J. Buchanan and R. S. Lewis (2006). "The elementary unit of storeoperated Ca2+ entry: local activation of CRAC channels by STIM1 at ER-plasma membrane junctions." J Cell Biol 174(6): 815-25.

Lynch, D. K., S. C. Winata, R. J. Lyons, W. E. Hughes, G. M. Lehrbach, V. Wasinger, G. Corthals, S. Cordwell and R. J. Daly (2003). "A Cortactin-CD2-associated protein 
(CD2AP) complex provides a novel link between epidermal growth factor receptor endocytosis and the actin cytoskeleton." J Biol Chem 278(24): 21805-13.

Mayer, B. J. (2001). "SH3 domains: complexity in moderation." J Cell Sci 114(Pt 7): 1253-63.

Mayer, B. J. and M. J. Eck (1995). "SH3 domains. Minding your p's and q's." Curr Biol 5(4): 364-7.

Mayer, B. J., M. Hamaguchi and H. Hanafusa (1988). "A novel viral oncogene with structural similarity to phospholipase C." Nature 332(6161): 272-5.

Melchers, F., A. Rolink, U. Grawunder, T. H. Winkler, H. Karasuyama, P. Ghia and J. Andersson (1995). "Positive and negative selection events during B lymphopoiesis." Curr Opin Immunol 7(2): 214-27.

Minegishi, Y., J. Rohrer, E. Coustan-Smith, H. M. Lederman, R. Pappu, D. Campana, A. C. Chan and M. E. Conley (1999). "An essential role for BLNK in human B cell development." Science 286(5446): 1954-7.

Mitchell, G. F. and J. F. A. P. Miller (1968). "Cell to cell interaction in the immune response. II. The source of hemolysin-forming cell in irradiated mice given bone marrow and thymus or thoracic duct lymphocytes." J.Exp.Med.(128): 821-837.

Molfetta, R., F. Belleudi, G. Peruzzi, S. Morrone, L. Leone, I. Dikic, M. Piccoli, L. Frati, M. R. Torrisi, A. Santoni and R. Paolini (2005). "CIN85 regulates the ligand-dependent endocytosis of the IgE receptor: a new molecular mechanism to dampen mast cell function." J Immunol 175(7): 4208-16.

Monks, C. R., B. A. Freiberg, H. Kupfer, N. Sciaky and A. Kupfer (1998). "Three-dimensional segregation of supramolecular activation clusters in T cells." Nature 395(6697): 82-6.

Morita, S., T. Kojima and T. Kitamura (2000). "Plat-E: an efficient and stable system for transient packaging of retroviruses." Gene Ther 7(12): 1063-6.

Mullis, K., F. Faloona, S. Scharf, R. Saiki, G. Horn and H. Erlich (1986). "Specific enzymatic amplification of DNA in vitro: the polymerase chain reaction." Cold Spring Harb Symp Quant Biol 51 Pt 1: 263-73.

Narita, T., F. Amano, K. Yoshizaki, N. Nishimoto, T. Nishimura, T. Tajima, H. Namiki and T. Taniyama (2001). "Assignment of SH3KBP1 to human chromosome band Xp22.1->p21.3 by in situ hybridization." Cytogenet Cell Genet 93(1-2): 133-4.

Narita, T., T. Nishimura, K. Yoshizaki and T. Taniyama (2005). "CIN85 associates with TNF receptor 1 via Src and modulates TNF-alpha-induced apoptosis." Exp Cell Res 304(1): 256-64.

Navarro, M. N., G. Nusspaumer, P. Fuentes, S. Gonzalez-Garcia, J. Alcain and M. L. Toribio (2007). "Identification of CMS as a cytosolic adaptor of the human pTalpha chain involved in pre-TCR function." Blood 110(13): 4331-40. 
Neumann, K., T. Oellerich, H. Urlaub and J. Wienands (2009). "The B-lymphoid Grb2 interaction code." Immunol Rev 232(1): 135-49.

Niiro, H. and E. A. Clark (2002). "Regulation of B-cell fate by antigen-receptor signals." Nat Rev Immunol 2(12): 945-56.

Niiro, H., S. Jabbarzadeh-Tabrizi, Y. Kikushige, T. Shima, K. Noda, S. Ota, H. Tsuzuki, Y. Inoue, Y. Arinobu, H. Iwasaki, S. Shimoda, E. Baba, H. Tsukamoto, T. Horiuchi, T. Taniyama and K. Akashi (2012). "CIN85 is required for Cbl-mediated regulation of antigen receptor signaling in human B cells." Blood 119(10): 2263-73.

Niiro, H., S. Jabbarzadeh-Tabrizi, Y. Kikushige, T. Shima, K. Noda, S. I. Ota, H. Tsuzuki, Y. Inoue, Y. Arinobu, H. Iwasaki, S. Shimoda, E. Baba, H. Tsukamoto, T. Horiuchi, T. Taniyama and K. Akashi "CIN85 is required for Cbl-mediated regulation of antigen receptor signaling in human $B$ cells." Blood.

Oellerich, T., V. Bremes, K. Neumann, H. Bohnenberger, K. Dittmann, H. H. Hsiao, M. Engelke, T. Schnyder, F. D. Batista, H. Urlaub and J. Wienands (2011). "The B-cell antigen receptor signals through a preformed transducer module of SLP65 and CIN85." Embo J 30(17): 3620-34.

Oellerich, T., M. Gronborg, K. Neumann, H. H. Hsiao, H. Urlaub and J. Wienands (2009). "SLP-65 phosphorylation dynamics reveals a functional basis for signal integration by receptor-proximal adaptor proteins." Mol Cell Proteomics 8(7): 1738-50.

Okada, T., A. Maeda, A. Iwamatsu, K. Gotoh and T. Kurosaki (2000). "BCAP: the tyrosine kinase substrate that connects $B$ cell receptor to phosphoinositide 3-kinase activation." Immunity 13(6): 817-27.

Paddison, P. J., M. Cleary, J. M. Silva, K. Chang, N. Sheth, R. Sachidanandam and G. J. Hannon (2004). "Cloning of short hairpin RNAs for gene knockdown in mammalian cells." Nat Methods 1(2): 163-7.

Pappu, R., A. M. Cheng, B. Li, Q. Gong, C. Chiu, N. Griffin, M. White, B. P. Sleckman and A. C. Chan (1999). "Requirement for B cell linker protein (BLNK) in B cell development." Science 286(5446): 1949-54.

Pasteur, L. (1880). "Sur les maladies virulentes, et en particulier sur la maladie appelee vulgairement cholera des poules." C. R. Hebd. Seances Acad. Sci. 90: 239.

Patterson, H. C., M. Kraus, Y. M. Kim, H. Ploegh and K. Rajewsky (2006). "The B cell receptor promotes $B$ cell activation and proliferation through a non-ITAM tyrosine in the Igalpha cytoplasmic domain." Immunity 25(1): 55-65.

Patterson, R. L., D. Boehning and S. H. Snyder (2004). "Inositol 1,4,5-trisphosphate receptors as signal integrators." Annu Rev Biochem 73: 437-65.

Pawson, T. and P. Nash (2003). "Assembly of cell regulatory systems through protein interaction domains." Science 300(5618): 445-52. 
Petrelli, A., G. F. Gilestro, S. Lanzardo, P. M. Comoglio, N. Migone and S. Giordano (2002). "The endophilin-CIN85-Cbl complex mediates ligand-dependent downregulation of CMet." Nature 416(6877): 187-90.

Porter, R. R. (1991). "Lecture for the Nobel Prize for physiology or medicine 1972: Structural studies of immunoglobulins. 1972." Scand J Immunol 34(4): 381-9.

Prakriya, M., S. Feske, Y. Gwack, S. Srikanth, A. Rao and P. G. Hogan (2006). "Orai1 is an essential pore subunit of the CRAC channel." Nature 443(7108): 230-3.

Rawlings, D. J., A. M. Scharenberg, H. Park, M. I. Wahl, S. Lin, R. M. Kato, A. C. Fluckiger, O. N. Witte and J. P. Kinet (1996). "Activation of BTK by a phosphorylation mechanism initiated by SRC family kinases." Science 271(5250): 822-5.

Reth, M. (1989). "Antigen receptor tail clue." Nature 338(6214): 383-4.

Reth, M. (1991). "Regulation of B-cell development by pre-B-cell receptors." Curr Biol 1(3): 198-9.

Reth, M. (1992). "Antigen receptors on B lymphocytes." Annu Rev Immunol 10: 97-121.

Reth, M. (2001). "Oligomeric antigen receptors: a new view on signaling for the selection of lymphocytes." Trends Immunol 22(7): 356-60.

Reth, M. and J. Wienands (1997). "Initiation and processing of signals from the B cell antigen receptor." Annu Rev Immunol 15: 453-79.

Rodriguez, R., M. Matsuda, A. Storey and M. Katan (2003). "Requirements for distinct steps of phospholipase Cgamma2 regulation, membrane-raft-dependent targeting and subsequent enzyme activation in B-cell signalling." Biochem J 374(Pt 1): 269-80.

Roos, J., P. J. DiGregorio, A. V. Yeromin, K. Ohlsen, M. Lioudyno, S. Zhang, O. Safrina, J. A. Kozak, S. L. Wagner, M. D. Cahalan, G. Velicelebi and K. A. Stauderman (2005). "STIM1, an essential and conserved component of store-operated Ca2+ channel function." J Cell Biol 169(3): 435-45.

Rowley, R. B., A. L. Burkhardt, H. G. Chao, G. R. Matsueda and J. B. Bolen (1995). "Syk protein-tyrosine kinase is regulated by tyrosine-phosphorylated Ig alpha/lg beta immunoreceptor tyrosine activation motif binding and autophosphorylation." $\underline{\mathrm{J} \text { Biol }}$ Chem 270(19): 11590-4.

Salisbury, J. L., J. S. Condeelis and P. Satir (1980). "Role of coated vesicles, microfilaments, and calmodulin in receptor-mediated endocytosis by cultured B lymphoblastoid cells." J Cell Biol 87(1): 132-41.

Sanchez, M., Z. Misulovin, A. L. Burkhardt, S. Mahajan, T. Costa, R. Franke, J. B. Bolen and M. Nussenzweig (1993). "Signal transduction by immunoglobulin is mediated through

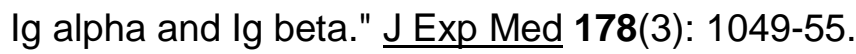

Sauer, K., J. Liou, S. B. Singh, D. Yablonski, A. Weiss and R. M. Perlmutter (2001). "Hematopoietic progenitor kinase 1 associates physically and functionally with the 
adaptor proteins B cell linker protein and SLP-76 in lymphocytes." $\underline{\mathrm{J} \text { Biol Chem }}$ 276(48): 45207-16.

Schamel, W. W. and M. Reth (2000a). "Monomeric and oligomeric complexes of the B cell antigen receptor." Immunity 13(1): 5-14.

Schamel, W. W. and M. Reth (2000b). "Stability of the B cell antigen receptor complex." Mol Immunol 37(5): 253-9.

Schmidt, M. H., B. Chen, L. M. Randazzo and O. Bogler (2003). "SETA/CIN85/Ruk and its binding partner AIP1 associate with diverse cytoskeletal elements, including FAKs,

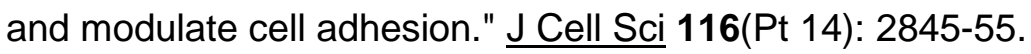

Schnyder, T., A. Castello, C. Feest, N. E. Harwood, T. Oellerich, H. Urlaub, M. Engelke, J. Wienands, A. Bruckbauer and F. D. Batista (2011). "B cell receptor-mediated antigen gathering requires ubiquitin ligase $\mathrm{Cbl}$ and adaptors $\mathrm{Grb} 2$ and Dok-3 to recruit dynein to the signaling microcluster." Immunity 34(6): 905-18.

Schreiner, G. F. and E. R. Unanue (1977). "Capping and the lymphocyte: models for membrane reorganization." J Immunol 119(5): 1549-51.

Selvy, P. E., R. R. Lavieri, C. W. Lindsley and H. A. Brown "Phospholipase D: enzymology, functionality, and chemical modulation." Chem Rev 111(10): 6064-119.

Shaw, A. C., R. N. Mitchell, Y. K. Weaver, J. Campos-Torres, A. K. Abbas and P. Leder (1990). "Mutations of immunoglobulin transmembrane and cytoplasmic domains: effects on intracellular signaling and antigen presentation." Cell 63(2): 381-92.

Shih, N. Y., J. Li, V. Karpitskii, A. Nguyen, M. L. Dustin, O. Kanagawa, J. H. Miner and A. S. Shaw (1999). "Congenital nephrotic syndrome in mice lacking CD2-associated protein." Science 286(5438): 312-5.

Shimokawa, N., K. Haglund, S. M. Holter, C. Grabbe, V. Kirkin, N. Koibuchi, C. Schultz, J. Rozman, D. Hoeller, C. H. Qiu, M. B. Londono, J. Ikezawa, P. Jedlicka, B. Stein, S. W. Schwarzacher, D. P. Wolfer, N. Ehrhardt, R. Heuchel, I. Nezis, A. Brech, M. H. Schmidt, H. Fuchs, V. Gailus-Durner, M. Klingenspor, O. Bogler, W. Wurst, T. Deller, M. H. de Angelis and I. Dikic (2010). "CIN85 regulates dopamine receptor endocytosis and governs behaviour in mice." Embo J 29(14): 2421-32.

Sohn, H. W., P. Tolar, T. Jin and S. K. Pierce (2006). "Fluorescence resonance energy transfer in living cells reveals dynamic membrane changes in the initiation of $B$ cell signaling." Proc Natl Acad Sci U S A 103(21): 8143-8.

Sohn, H. W., P. Tolar and S. K. Pierce (2008). "Membrane heterogeneities in the formation of B cell receptor-Lyn kinase microclusters and the immune synapse." J Cell Biol 182(2): 367-79. 
Song, W., H. Cho, P. Cheng and S. K. Pierce (1995). "Entry of B cell antigen receptor and antigen into class II peptide-loading compartment is independent of receptor crosslinking." J Immunol 155(9): 4255-63.

Soubeyran, P., K. Kowanetz, I. Szymkiewicz, W. Y. Langdon and I. Dikic (2002). "Cbl-CIN85endophilin complex mediates ligand-induced downregulation of EGF receptors." Nature 416(6877): 183-7.

Spector, I., N. R. Shochet, D. Blasberger and Y. Kashman (1989). "Latrunculins--novel marine macrolides that disrupt microfilament organization and affect cell growth: I. Comparison with cytochalasin D." Cell Motil Cytoskeleton 13(3): 127-44.

Stace, C. L. and N. T. Ktistakis (2006). "Phosphatidic acid- and phosphatidylserine-binding proteins." Biochim Biophys Acta 1761(8): 913-26.

Stahl, M. L., C. R. Ferenz, K. L. Kelleher, R. W. Kriz and J. L. Knopf (1988). "Sequence similarity of phospholipase $C$ with the non-catalytic region of src." Nature 332(6161): 269-72.

Stoddart, A., M. L. Dykstra, B. K. Brown, W. Song, S. K. Pierce and F. M. Brodsky (2002). "Lipid rafts unite signaling cascades with clathrin to regulate BCR internalization." Immunity 17(4): 451-62.

Stoddart, A., A. P. Jackson and F. M. Brodsky (2005). "Plasticity of B cell receptor internalization upon conditional depletion of clathrin." Mol Biol Cell 16(5): 2339-48.

Stork, B., M. Engelke, J. Frey, V. Horejsi, A. Hamm-Baarke, B. Schraven, T. Kurosaki and J. Wienands (2004). "Grb2 and the non-T cell activation linker NTAL constitute a $\mathrm{Ca}(2+)$-regulating signal circuit in B lymphocytes." Immunity 21(5): 681-91.

Su, Y. W. and H. Jumaa (2003). "LAT links the pre-BCR to calcium signaling." Immunity 19(2): 295-305.

Su, Y. W., Y. Zhang, J. Schweikert, G. A. Koretzky, M. Reth and J. Wienands (1999). "Interaction of SLP adaptors with the SH2 domain of Tec family kinases." Eur J Immunol 29(11): 3702-11.

Sugawara, H., M. Kurosaki, M. Takata and T. Kurosaki (1997). "Genetic evidence for involvement of type 1, type 2 and type 3 inositol 1,4,5-trisphosphate receptors in signal transduction through the B-cell antigen receptor." Embo J 16(11): 3078-88.

Swamy, M., Y. Kulathu, S. Ernst, M. Reth and W. W. Schamel (2006). "Two dimensional Blue Native-/SDS-PAGE analysis of SLP family adaptor protein complexes." Immunol Lett 104(1-2): 131-7.

Szymkiewicz, I., K. Kowanetz, P. Soubeyran, A. Dinarina, S. Lipkowitz and I. Dikic (2002). "CIN85 participates in Cbl-b-mediated down-regulation of receptor tyrosine kinases." J Biol Chem 277(42): 39666-72. 
Takata, M., H. Sabe, A. Hata, T. Inazu, Y. Homma, T. Nukada, H. Yamamura and T. Kurosaki (1994). "Tyrosine kinases Lyn and Syk regulate B cell receptor-coupled Ca2+ mobilization through distinct pathways." Embo J 13(6): 1341-9.

Take, H., S. Watanabe, K. Takeda, Z. X. Yu, N. Iwata and S. Kajigaya (2000). "Cloning and characterization of a novel adaptor protein, CIN85, that interacts with c-Cbl." Biochem Biophys Res Commun 268(2): 321-8.

Tanner, M. J., W. Hanel, S. L. Gaffen and X. Lin (2007). "CARMA1 coiled-coil domain is involved in the oligomerization and subcellular localization of CARMA1 and is required for T cell receptor-induced NF-kappaB activation." J Biol Chem 282(23): 17141-7.

Taylor, R. B., W. P. Duffus, M. C. Raff and S. de Petris (1971). "Redistribution and pinocytosis of lymphocyte surface immunoglobulin molecules induced by antiimmunoglobulin antibody." Nat New Biol 233(42): 225-9.

Tibaldi, E. V. and E. L. Reinherz (2003). "CD2BP3, CIN85 and the structurally related adaptor protein CMS bind to the same CD2 cytoplasmic segment, but elicit divergent functional activities." Int Immunol 15(3): 313-29.

Tonegawa, S. (1983). "Somatic generation of antibody diversity." Nature 302(5909): 575-81.

Tossidou, I., R. Niedenthal, M. Klaus, B. Teng, K. Worthmann, B. L. King, K. J. Peterson, H. Haller and M. Schiffer (2011). "CD2AP regulates SUMOylation of CIN85 in podocytes." Mol Cell Biol.

Treanor, B., D. Depoil, A. Gonzalez-Granja, P. Barral, M. Weber, O. Dushek, A. Bruckbauer and F. D. Batista (2009). "The membrane skeleton controls diffusion dynamics and signaling through the B cell receptor." Immunity 32(2): 187-99.

Tsubata, T. and M. Reth (1990). "The products of pre-B cell-specific genes (lambda 5 and VpreB) and the immunoglobulin mu chain form a complex that is transported onto the cell surface." J Exp Med 172(3): 973-6.

Venkitaraman, A. R., G. T. Williams, P. Dariavach and M. S. Neuberger (1991). "The B-cell antigen receptor of the five immunoglobulin classes." Nature 352(6338): 777-81.

Verdier, F., T. Valovka, A. Zhyvoloup, L. B. Drobot, V. Buchman, M. Waterfield and I. Gout (2002). "Ruk is ubiquitinated but not degraded by the proteasome." Eur J Biochem 269(14): 3402-8.

Vig, M., C. Peinelt, A. Beck, D. L. Koomoa, D. Rabah, M. Koblan-Huberson, S. Kraft, H. Turner, A. Fleig, R. Penner and J. P. Kinet (2006). "CRACM1 is a plasma membrane protein essential for store-operated Ca2+ entry." Science 312(5777): 1220-3.

Viola, A. and N. Gupta (2007). "Tether and trap: regulation of membrane-raft dynamics by actin-binding proteins." Nat Rev Immunol 7(11): 889-96. 
von Behring, E. and S. Kitasato (1890). "Ueber das Zustandekommen der DiphterieImmunitaet und der Tetanus-Immunitaet bei Thieren." Dtsch. Med. Wochenschr 16: 1113-1114.

Vos, Q., A. Lees, Z. Q. Wu, C. M. Snapper and J. J. Mond (2000). "B-cell activation by T-cellindependent type 2 antigens as an integral part of the humoral immune response to pathogenic microorganisms." Immunol Rev 176: 154-70.

Wang, L. D. and M. R. Clark (2003). "B-cell antigen-receptor signalling in lymphocyte development." Immunology 110(4): 411-20.

Watanabe, S., H. Take, K. Takeda, Z. X. Yu, N. Iwata and S. Kajigaya (2000). "Characterization of the CIN85 adaptor protein and identification of components involved in CIN85 complexes." Biochem Biophys Res Commun 278(1): 167-74.

Weber, M., B. Treanor, D. Depoil, H. Shinohara, N. E. Harwood, M. Hikida, T. Kurosaki and F. D. Batista (2008). "Phospholipase C-gamma2 and Vav cooperate within signaling microclusters to propagate $B$ cell spreading in response to membrane-bound antigen." J Exp Med 205(4): 853-68.

Wienands, J., F. Freuler and G. Baumann (1995). "Tyrosine-phosphorylated forms of Ig beta, CD22, TCR zeta and HOSS are major ligands for tandem SH2 domains of Syk." Int Immunol 7(11): 1701-8.

Wienands, J., J. Hombach, A. Radbruch, C. Riesterer and M. Reth (1990). "Molecular components of the $B$ cell antigen receptor complex of class $\lg D$ differ partly from those of IgM." Embo J 9(2): 449-55.

Wienands, J., O. Larbolette and M. Reth (1996). "Evidence for a preformed transducer complex organized by the B cell antigen receptor." Proc Natl Acad Sci U S A 93(15): 7865-70.

Wienands, J., J. Schweikert, B. Wollscheid, H. Jumaa, P. J. Nielsen and M. Reth (1998). "SLP-65: a new signaling component in B lymphocytes which requires expression of the antigen receptor for phosphorylation." J Exp Med 188(4): 791-5.

Winding, P. and M. W. Berchtold (2001). "The chicken B cell line DT40: a novel tool for gene disruption experiments." J Immunol Methods 249(1-2): 1-16.

Wong, J., M. Ishiai, T. Kurosaki and A. C. Chan (2000). "Functional complementation of BLNK by SLP-76 and LAT linker proteins." J Biol Chem 275(42): 33116-22.

Wu, T. T. and E. A. Kabat (1970). "An analysis of the sequences of the variable regions of Bence Jones proteins and myeloma light chains and their implications for antibody complementarity." J Exp Med 132(2): 211-50.

Xu, P., J. Lu, Z. Li, X. Yu, L. Chen and T. Xu (2006). "Aggregation of STIM1 underneath the plasma membrane induces clustering of Orai1." Biochem Biophys Res Commun 350(4): 969-76. 
Xu, S., J. E. Tan, E. P. Wong, A. Manickam, S. Ponniah and K. P. Lam (2000). "B cell development and activation defects resulting in xid-like immunodeficiency in BLNK/SLP-65-deficient mice." Int Immunol 12(3): 397-404.

Yamanashi, Y., T. Kakiuchi, J. Mizuguchi, T. Yamamoto and K. Toyoshima (1991). "Association of B cell antigen receptor with protein tyrosine kinase Lyn." $\underline{\text { Science }}$ 251(4990): 192-4.

Zhang, J., X. Zheng, X. Yang and K. Liao (2009). "CIN85 associates with endosomal membrane and binds phosphatidic acid." Cell Res 19(6): 733-46.

Zhang, S. L., Y. Yu, J. Roos, J. A. Kozak, T. J. Deerinck, M. H. Ellisman, K. A. Stauderman and M. D. Cahalan (2005). "STIM1 is a Ca2+ sensor that activates CRAC channels and migrates from the Ca2+ store to the plasma membrane." Nature 437(7060): 9025.

Zhang, W., J. Sloan-Lancaster, J. Kitchen, R. P. Trible and L. E. Samelson (1998). "LAT: the ZAP-70 tyrosine kinase substrate that links $T$ cell receptor to cellular activation." $\underline{\text { Cell }}$ 92(1): 83-92.

Zhao, C., G. Du, K. Skowronek, M. A. Frohman and D. Bar-Sagi (2007). "Phospholipase D2generated phosphatidic acid couples EGFR stimulation to Ras activation by Sos." Nat Cell Biol 9(6): 706-12. 
Curriculum vitae 